Werther Alexandre de Oliveira Serralheiro

Arquitetura de Controle de Movimento para um Robô Móvel sobre Rodas visando Otimização Energética

São Paulo

2018 

Werther Alexandre de Oliveira Serralheiro

\section{Arquitetura de Controle de Movimento para um Robô Móvel sobre Rodas visando Otimização Energética \\ (Versão Corrigida)}

Tese apresentada à Escola Politécnica da Universidade de São Paulo para obtenção do título de doutor em Ciências

São Paulo

2018 
ESPAÇO RESERVADO PARA O

RELATÓRIO DE DEFESA 
Werther Alexandre de Oliveira Serralheiro

\section{Arquitetura de Controle de Movimento para um Robô Móvel sobre Rodas visando Otimização Energética (Versão Corrigida)}

Tese apresentada à Escola Politécnica da Universidade de São Paulo para obtenção do título de doutor em Ciências

Área de Concentração:

Engenharia Mecânica

Orientador:

Prof. Dr. Newton Maruyama

São Paulo

2018 
Este exemplar foi revisado e corrigido em relação à versão original, sob responsabilidade única do autor e com a anuência de seu orientador.

São Paulo, de de

Assinatura do autor:

Assinatura do orientador:

Catalogação-na-publicação

Serralheiro, Werther Alexandre de Oliveira

Arquitetura de Controle de Movimento para um Robô Móvel sobre Rodas visando Otimização Energética / W. A. O. Serralheiro -- versão corr. -- São Paulo, 2018.

$165 \mathrm{p}$.

Tese (Doutorado) - Escola Politécnica da Universidade de São Paulo. Departamento de Engenharia Mecânica.

1.Robôs Móveis Inteligentes 2.Otimização Convexa 3.Mecatrônica I.Universidade de São Paulo. Escola Politécnica. Departamento de Engenharia Mecânica II.t. 
À Samanta e à Bárbara. 



\section{AGRADECIMENTOS}

Minhas convicções como educador me levam a crer que todas as pessoas que participaram de forma direta ou indireta na minha formação durante toda a minha vida são importantes e, portanto, merecem meus sinceros agradecimentos. Como a tarefa de agradecer a todos é, assim diria meu orientador, "hercúlea", vou me privar de fazer meus sinceros agradecimentos àqueles que contribuiriam especificamente para a realização desta etapa da minha vida acadêmica.

Primeiramente à hoje Mag. Reitora Maria Clara Kaschny Schneider e sua equipe, assim como os professores Jurandir Yanagihara e Gilberto Martha de Souza que não mediram esforços para a realização do DINTER entre o IFSC e a EPUSP.

Aos meus colegas do Departamento de Eletromecânica do Campus Araranguá, gratidão por viabilizarem a minha participação nas disciplinas do Doutorado em Florianópolis, dando suporte logístico e acolhendo parte de minha carga horária como docente. Gratidão especial ao agora ex-colega Fabrício Saggin pela parceria nas pesquisas.

Um abraço carinhoso para meus colegas da turma do DINTER: Cláudio Weber, Edson Watanabe, Eduardo Suzuki, Julio Silva, Marcos Pisching, Alencar Migliavacca e Elenira Vilela; especialmente aos dois últimos que tiveram uma participação especial em minha estadia na cidade de São Paulo em 2014.

Aos colegas do Laboratório de Sistemas Embarcados - ESL, em especial ao professor Celso Furukawa, ao pesquisador Douglas Santana e aos bolsistas Vitor Matosinho e Edson Yuji - pela companhia diária, confraternização, debates e colaboração acadêmica.

Em minha avaliação de qualificação obtive orientações dos membros da banca, os professores Eduardo Tannuri e Diego Cólon, no sentido de buscar soluções com Otimização Convexa que foram fundamentais para a conclusão deste trabalho. Amplio este agradecimento ao acadêmico Guilherme Scabin pelas conversas sobre o tema e ao sempre companheiro professor Anderson Perez pela parceria nas pesquisas.

Agradecimentos especiais para o colega e professor Eduardo Camponogara. Sua dedicação, paciência e colaboração na resolução numérica dos problemas de otimização presentes neste trabalho foram de fundamental importância para minha formação.

Durante o processo de doutoramento, não deixei de atuar como educador. Isso foi importante para que eu continuasse motivado. Não posso deixar de agradecer aos meus alunos orientandos que mantiveram vivas as minhas espectativas quanto à tarefa de orientar pesquisa científica e tecnológica. Abraços carinhosos ao meus pupilos Renan Darós, André Barbosa, Elizandro Fagundes, Jucemar Paes e Lucas dos Santos. 
Meu orientador, professor Newton Maruyama, merece um agradecimento mais do que especial. Ele acreditou na minha proposta, sem nunca deixar de ser crítico a ela. Ele me deixou livre para seguir meus próprios caminhos, sem nunca me deixar sozinho. Suas conversas de forma serena, a maioria das vezes com poucas palavras, foram significativas. Levo comigo deste processo de doutoramento este estilo de orientação para minha vida profissional. Maru, obrigado!

A finalização deste documento teve relevantes contribuições dos membros da banca de doutoramento, os já citados professores Newton Maruyama, Eduardo Tannuri e Diego Cólon, além da participação dos professores Eduardo Godoy (UNESC Sorocaba) e Teodiano Freire Bastos Filho (UFES), cujas participações foram de extrema importância para minha formação.

Por fim deixo agradecimentos aos mais importantes. São simplesmente a razão de tudo isso. É o que me motivou a entrar e permanecer firme neste processo longo que é o doutoramento. Agradeço minha esposa Samanta, minha filhotinha Bárbara, minha irmã Wendie e minha sobrinha Rafaela por compreender os finais de semana sem passeios, os dias em completo isolamento em meu "QG", a minha corriqueira impaciência com os assuntos do dia-a-dia, minha concentração e minhas alucinações por vezes quase insanas a respeito do tema de minha Tese. Para elas, além de agradecimento, eu peço desculpas por minha ausência nestes quatro anos - vou pagar em dobro daqui por diante. 
"Se algum de vocês tem falta de sabedoria, peça-a a Deus, que a todos dá livremente, de boa vontade; e lhe será concedida." (Bíblia Sagrada, Tiago 1:5) 



\section{RESUMO}

SERRALHEIRO, Werther Alexandre de Oliveira. Arquitetura de Controle de Movimento para um Robô Móvel sobre Rodas visando Otimização Energética.2018. 165 p. Tese (Doutorado em Engenharia Mecânica) - Escola Politécnica, Universidade de São Paulo, São Paulo, 2018.

Este trabalho apresenta uma arquitetura de controle de movimento entre duas posturas distintas para um robô móvel sob rodas com acionamento diferencial em um ambiente estruturado e livre de obstáculos. O conceito clássico de eficiência foi utilizado para a definição das estratégias de controle: um robô se movimenta de forma eficiente quando realiza a tarefa determinada no menor tempo e utilizando menor quantidade energética. A arquitetura proposta é um recorte do modelo de Controle Hierárquico Aninhado (NHC), composto por três níveis de abstração: (i) Planejamento de Caminho, (ii) Planejamento de Trajetória e (iii) Rastreamento de Trajetória. O Planejamento de Caminho proposto suaviza uma geodésica Dubins - o caminho mais eficiente - por uma Spline Grampeada para que este caminho seja definido por uma curva duplamente diferenciável. Uma transformação do espaço de configuração do robô é realizada. O Planejamento de Trajetória é um problema de otimização convexa na forma de Programação Cônica de Segunda Ordem, cujo objetivo é uma função ponderada entre tempo e energia. Como o tempo de percurso e a energia total consumida pelo robô possui uma relação hiperbólica, um algoritmo de sintonia do coeficiente de ponderação entre estas grandezas é proposta. Por fim, um Rastreador de Trajetória de dupla malha baseado em linearização entrada-saída e controle PID é proposto, e obteve resultados satisfatórios no rastreamento do caminho pelo robô.

Palavras-chave: Robô Móvel, Arquitetura de Controle, Controle de Movimento, Otimização Convexa 



\section{ABSTRACT}

SERRALHEIRO, Werther Alexandre de Oliveira. Arquitetura de Controle de Movimento para um Robô Móvel sobre Rodas visando Otimização Energética.2018. 165 p. Tese (Doutorado em Engenharia Mecânica) - Escola Politécnica, Universidade de São Paulo, São Paulo, 2018.

This work presents a motion control architecture between two different positions for a differential driven wheeled mobile robot in a obstacles free structured environment. The classic concept of efficiency was used to define the control strategies: a robot moves efficiently when it accomplishes the determined task in the shortest time and using less amount of energy. The proposed architecture is a clipping of the Nested Hierarchical Controller (NHC) model, composed of three levels of abstraction: (i) Path Planning, (ii) Trajectory Planning and (iii) Trajectory Tracking. The proposed Path Planning smoothes a geodesic Dubins - the most efficient path - by a Clamped Spline as this path is defined by a twice differentiable curve. A transformation of the robot configuration space is performed. The Trajectory Planning is a convex optimization problem in the form of Second Order Cone Programming, whose objective is a weighted function between time and energy. As the travel time and the total energy consumed by the robot has a hyperbolic relation, a tuning algorithm to the weighting is proposed. Finnaly, a dual-loop Trajectory Tracker based on input-output feedback linearization and PID control is proposed, which obtained satisfactory results in tracking the path by the robot.

Keywords: Mobile Robot, Control Architecture, Motion Control, Convex Optimization 



\section{LISTA DE ILUSTRAÇÕES}

Figura 1 - Diagrama da arquitetura NHC. . . . . . . . . . . . . . 34

Figura 2 - Um WMR e seu caminho em um ambiente estruturado. . . . . . . . . 35

Figura 3 - O problema de movimento entre duas posturas. . . . . . . . . . 36

Figura 4 - O robô diferencial e seus sistemas referenciais. . . . . . . . . . . . . . . 42

Figura 5 - Uma roda fixa convencional e seus parâmetros. . . . . . . . . . . . . 43

Figura 6 - Relação geométrica entre os vetores de velocidade do robô. . . . . . . . 45

Figura 7 - Problemas clássicos de controle de movimento de robôs móveis encontrados na literatura. . . . . . . . . . . . . . . . . . . . 4 47

Figura 8 - Uma ilustração simplificada do problema de planejamento de caminho. 51

Figura 9 - Exemplos simples de convexidade de conjuntos. . . . . . . . . . 55

Figura 10 - Exemplo simples de cone convexo. . . . . . . . . . . . . . . 55

Figura 11 - Gráfico de uma função convexa. . . . . . . . . . . . . . . . 56

Figura 12 - Ilustração da condição de primeira ordem para a convexidade. . . . . . 57

Figura 13 - A função de barreira logarítmica. . . . . . . . . . . . . . . . . . . 61

Figura 14 - O robô uniciclo diferencial, seus sistemas referenciais e sua composição de forças. . . . . . . . . . . . . . . . . . . . 6 67

Figura 15 - A posição $p$ do robô uniciclo diferencial e seu caminho de referência $s$ parametrizada em $\tau(t) \ldots \ldots \ldots$. . . . . . . . . . . 71

Figura $16-a(\tau)$ é constante por partes para $\tau \in[0,1] \ldots \ldots$. . . . . . . . 76

Figura $17-b(\tau)$ é linear por partes para $\tau \in[0,1] \ldots \ldots \ldots$. . . . . . . 77

Figura 18 - Gráfico de uma curva $\Gamma$ no plano cartesiano. . . . . . . . . . . . 83

Figura 19 - Gráfico dos componentes do caminho $\Gamma$ medidos no tempo médio discreto $\overline{\mathbf{s}}_{i}$ e suas derivadas de primeira e segunda ordem. . . . . . . . . . . . 84

Figura 20 - Gráfico dos componentes do caminho $\Gamma$ gerados pela estimação das derivadas de segunda ordem pelo método de Runge-Kutta. . . . . . . . 84

Figura 21 - Gráfico dos pontos no plano cartesiano gerados pela estimação das derivadas de segunda ordem pelo método de Runge-Kutta. . . . . . . . 85

Figura 22 - Gráficos de resposta da otimização para $\mu=100, N=200$. . . . . . . 86

Figura 23 - Tempo de processamento versus número de discretização $N$ para os diferentes algoritmos de otimização. . . . . . . . . . . . . . . . . . 87

Figura 24 - Status final da otimização versus número de discretização $N$ para os diferentes algoritmos. . . . . . . . . . . . . . . . . 88

Figura 25 - Gráficos de resposta para $N=200$, considerando o status final da otimização. . . . . . . . . . . . . . . . . . . . . . . 89

Figura 26 - Resultado do Tempo final e da Energia total para os diferentes algoritmos de otimização. . . . . . . . . . . . . . . . . . . . 9 90 
Figura 27 - Resposta da otimização para diferentes valores de $\mu$. . . . . . . . . . 91

Figura 28 - Caminho $\Gamma$ discretizado com $N=500$. . . . . . . . . . . . . . 92

Figura 29 - Gráfico de Pareto e suas regiões com valores contínuos de $\mu$. . . . . . 93

Figura 30 - Gráficos de Pareto em escala linear e logarítmica. . . . . . . . . . . . . 94

Figura 31 - Gráficos das equações exponenciais entre $T_{f}$ e $E_{t}$ para diferentes valores

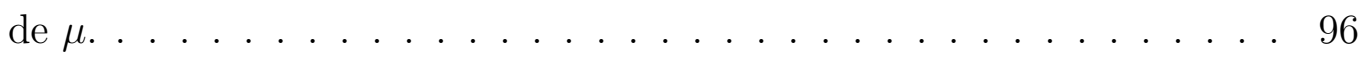

Figura 32 - Gráficos $T_{f}$ vs $\mu$ em escala linear e logarítmica, . . . . . . . . . . . 97

Figura 33 - Gráficos das equações exponenciais entre $T_{f}$ e $\mu$ para diferentes valores

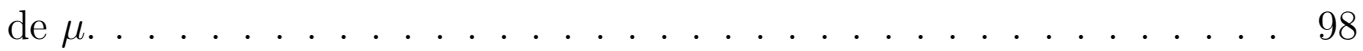

Figura 34 - Gráficos de Pareto com algumas sintonias de $\mu$ em função de $\Lambda$. . . . . 101

Figura 35 - Curvas de Dubins do tipo CSC candidatas à geodésica. . . . . . . . . . 106

Figura 36 - Curva Dubins e suas derivadas para $N=50$. . . . . . . . . . 107

Figura 37 - Curva Dubins e suas derivadas para $N=100$. . . . . . . . . . 107

Figura 38 - Curva Dubins e suas derivadas para $N=200$. . . . . . . . . . . 108

Figura 39 - Curva Dubins e suas derivadas para $N=500$. . . . . . . . . . 108

Figura 40 - Curva Dubins com $n+1=8$ waypoints. . . . . . . . . . . . . . 111

Figura 41 - Interpolação spline cúbica grampeada. . . . . . . . . . . . . . . . . 112

Figura 42 - Componentes cartesianos das Curvas Dubins original e suavizada . . . 116

Figura 43 - Curva Dubins suavizada. . . . . . . . . . . . . . . 117

Figura 44 - Componentes de $\overline{\mathbf{s}}_{i}$ e suas derivadas para a curva Dubins suavizada . . 118

Figura 45 - Resposta do algoritmo SOCP para a curva Dubins suavizada. . . . . . 119

Figura 46 - Curva Dubins suavizada tipo $R S R$ e suas componentes de $\overline{\mathbf{s}}_{i} \ldots \ldots$. . 120

Figura 47 - Curva Dubins suavizada tipo $R S L$ e suas componentes de $\overline{\mathbf{s}}_{i} \ldots \ldots$. . 121

Figura 48 - Curva $\Gamma$ e suas derivadas para a otimização, $N=500$. . . . . . . . 124

Figura 49 - Gráficos de valores de tensão ótima para a curva $\Gamma$, com $\mu=100$. . . 125

Figura 50 - Controle em malha aberta. . . . . . . . . . . . . . . . . 125

Figura 51 - Gráficos de resposta em malha aberta para a curva $\Gamma$. . . . . . . . . 126

Figura 52 - Gráficos de resposta em malha aberta para alguns tipos de curvas . . . 127

Figura 53 - Modelo de rastreamento de trajetória. . . . . . . . . . . . . . . . . 128

Figura 54 - Diagrama de blocos do controle de dupla malha. . . . . . . . . . . . . 131

Figura 55 - Rastreamento FBL+PID no plano em uma Dubins Suave tipo LSL. . . 132

Figura 56 - Intervalo de integração gerada pelo SOCP para a Dubins Suave tipo LSL.133

Figura 57 - Tensões de controle para o rastreamento FBL+PID em ambos os motores do robô. . . . . . . . . . . . . . . . . . . . . . 133

Figura 58 - Erros de rastreamento nas coordenadas alternativas em uma Dubins Suave tipo $L S L$. . . . . . . . . . . . . . . . . . . . . . . . . . . . . . . 134

Figura 59 - Velocidades linear e angular para o rastreamento FBL+PID. . . . . . . 134

Figura 60 - Rastreamento FBL+PID no plano em uma Dubins Suave tipo RSR. . 135 
Figura 61 - Erros de rastreamento nas coordenadas alternativas em uma Dubins

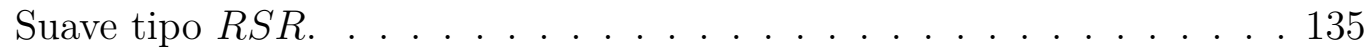

Figura 62 - Rastreamento FBL+PID no plano em uma Dubins Suave tipo RSL. . 136 Figura 63 - Erros de rastreamento nas coordenadas alternativas em uma Dubins Suave tipo $R S L \ldots \ldots \ldots \ldots \ldots \ldots$

Figura 64 - Diagrama de blocos da Arquitetura de Controle de Movimento. . . . . 141 



\section{LISTA DE TABELAS}

Tabela 1 - Erro médio de posição da estimativa por Runge-Kutta. . . . . . . . . . 85

Tabela 2 - Coeficientes $\alpha$ e $\beta$ calculados para alguns valores de $\mu \ldots$. . . . . 96

Tabela 3 - Coeficientes $\nu$ e $\kappa$ calculados para alguns valores de $\mu$. . . . . . . . 98

Tabela 4 - Resultados do Algoritmo de sintonia do coeficiente de ponderação $\mu$ e seu erro relativo à otimização para alguns valores de $\Lambda$. . . . . . . . . 100 



\section{LISTA DE ALGORITMOS}

1 Método de Barreira . . . . . . . . . . . . . . . . . . 63

2 Método do Ponto Interior Primal-Dual . . . . . . . . . . . . . . . . . 65

3 Transformação de coordenadas . . . . . . . . . . . . . . . . . . . . 83

4 Sintonia do coeficiente $\mu \ldots \ldots \ldots$. . . . . . . . . . . . . . 99 



\section{LISTA DE ABREVIATURAS E SIGLAS}

\begin{tabular}{|c|c|}
\hline $\mathrm{CP}$ & convex programming \\
\hline CSC & curve - straight - curve \\
\hline CVX & convex (optimization) \\
\hline DDOF & differentiable degrees of freedom \\
\hline $\mathrm{DOF}$ & degrees of freedom \\
\hline FBL & feedback linearization \\
\hline $\mathrm{KKT}$ & Karush-Kuhn-Tucker (conditions) \\
\hline LP & linear programming \\
\hline LRL & left - right - left \\
\hline LSL & left - straight - left \\
\hline LSR & left - straight - right \\
\hline $\mathrm{NHC}$ & nested hierarchical controller \\
\hline PI & (controle) proporcional integral \\
\hline PID & (controle) proporcional integral derivativo \\
\hline QCQP & quadratic constrained quadratic programming \\
\hline QP & quadratic programming \\
\hline RLR & right - left - right \\
\hline RSL & right - straight - left \\
\hline RSR & right - straight - right \\
\hline SDP & semi-definite programming \\
\hline SI & sistema internacional de unidades de medidas \\
\hline SOCP & second order cone programming \\
\hline WMR & wheeled mobile robot \\
\hline
\end{tabular}





\section{LISTA DE SÍMBOLOS}

\section{Alfabeto Romano}

$a$

$B$

$b$

C

$c \ldots f$

$e_{(.)}$

F

$i, j$

$J$

$K_{m}$

$K_{(.)}$

m

$N$

O

P

$r$

$T$

$t$

$T_{f}$

$u$

$v$

$x$

$X_{0}, Y_{0}$ aceleração do robô no tempo virtual

largura do robô

quadrado da velocidade do robô no tempo virtual

Ponto Cenoura

variáveis auxiliares para a SOCP

erro da variável (.) em relação à sua referência

módulo de um vetor de força

índices discretos

momento de inércia do robô

constante de torque do motor elétrico

ganho

massa do robô

quantidade de elementos discretos

origem do sistema de coordenada inercial

centro de rotação do robô

raio da roda do robô

torque

tempo

tempo final

tensão elétrica aplicada ao motor da roda do robô

velocidade linear do robô na direção de $X_{R}$

coordenada cartesiana do robô na direção $X_{0}$

eixos do sistema de coordenadas inercial 


$\begin{array}{ll}x_{0}, y_{0} & \text { posição inicial do robô } \\ X_{P}, Y_{P} & \text { eixos do sistema de coordenadas do robô } \\ y & \text { coordenada cartesiana do robô na direção } Y_{0} \\ \mathbf{F} & \text { manção matricial Hessiana } \\ \mathbf{H} & \text { matriz de ganhos } \\ \mathbf{K} & \text { matriz de inércia } \\ \mathbf{M} & \text { matriz de rotação da entrada } \\ \mathbf{R} & \text { vetor posição do robô } \\ \mathbf{p} & \text { vetor de coordenadas generalizadas alternativas } \\ \mathbf{q} & \text { caminho parametrizado geometricamente } \\ \mathbf{s} & \end{array}$

\section{Alfabeto Grego}

$\alpha$

$\beta$

$\Gamma$

$\gamma$

$\delta$

$\varepsilon$

$\theta$

$\iota$

$\kappa$

$\Lambda$

$\mu$

$\nu$

$\rho$ coeficiente exponencial de $T_{f}$ em relação a $E_{t}$

coeficiente multiplicativo de $T_{f}$ em relação a $E_{t}$

curva hipotética

avanço do robô

variação

erro

coordenada angular do robô

variável de integração no tempo

coeficiente multiplicativo de $T_{f}$ em relação a $\mu$

coeficiente de normalização entre $E_{t}$ e $T_{f}$

coeficiente de ponderação tempo-energia

coeficiente exponencial de $T_{f}$ em relação a $\mu$

raio mínimo da curva Dubins 


$\begin{array}{ll}\varrho & \text { hiato geométrico } \\ \tau & \text { parâmetro tempo virtual } \\ \omega & \text { velocidade angular do robô } \\ \boldsymbol{\xi} & \text { vetor postura do robô }\end{array}$

\section{Simbologia Especial}

$\begin{array}{ll}\mathcal{J} & \text { função objetivo } \\ \tilde{\mathcal{J}} & \text { função objetivo limitado } \\ \mathcal{C} & \text { conjunto convexo } \\ \mathbb{R} & \text { conjunto dos números reais } \\ \nabla & \text { gradiente } \\ (\cdot) & \text { derivada em relação ao tempo } t \\ (\cdot)^{\prime} & \text { derivada em relação ao tempo virtual } \tau \\ (\cdot)_{c} & \text { relativo ao Ponto Cenoura } \\ (\cdot)_{p},(\cdot)_{P} & \text { relativo à posição do robô } \\ (\cdot)_{q} & \text { relativo à configuração alternativa do robô } \\ (\cdot)_{\xi} & \text { relativo à postura do robô } \\ (\cdot)_{\text {max }} & \text { valor máximo de uma variável } \\ (\cdot)_{\text {min }} & \text { valor mínimo de uma variável } \\ (\cdot)_{l} & \text { relativo à roda esquerda à roda direita } \\ (\cdot)_{r} & \end{array}$





\section{SUMÁRIO}

INTRODUÇÃO $\ldots \ldots \ldots \ldots \ldots \ldots$

$1.1 \quad$ Escopo e Problema de Pesquisa $\ldots \ldots \ldots \ldots 33$

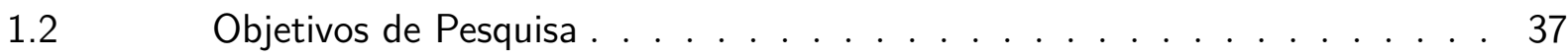

$1.3 \quad$ Contribuições da Tese $\ldots \ldots \ldots$. . . . . . . . . . . . 38

$1.4 \quad$ Organização do Texto $\ldots \ldots \ldots \ldots \ldots$

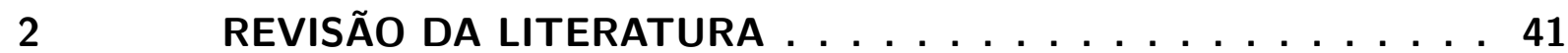

$2.1 \quad$ Cinemática de Robôs Móveis . . . . . . . . . . . . . . . . . . 41

2.1.1 Modelo cinemático do robô uniciclo diferencial . . . . . . . . . . . . . 41

$2.2 \quad$ Controle de Movimento de Robôs Móveis . . . . . . . . . . . . . . . . 46

2.2.1 Controlabilidade para o Problema de Rastreamento de Trajetória . . . . . . 49

$2.3 \quad$ Planejamento de Trajetória . . . . . . . . . . . . . . . . . 51

2.3.1 Planejamento ótimo de trajetória . . . . . . . . . . . . . 53

$2.4 \quad$ Otimização Convexa . . . . . . . . . . . . . . . . . . . 54

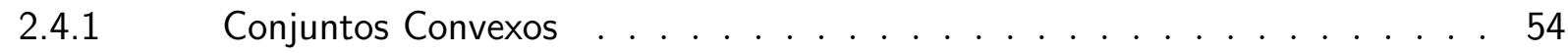

2.4.2 Cones Convexos . . . . . . . . . . . . . . . . . . . 55

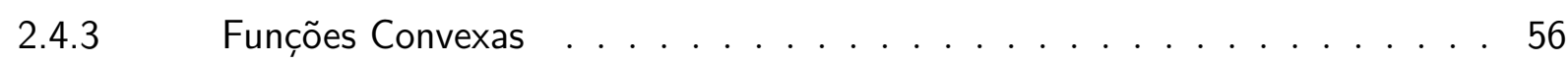

2.4.4 Problemas de Otimização Convexa . . . . . . . . . . . . . . . . . . 57

2.4.5 Método do Ponto Interior . . . . . . . . . . . . . . . . . 60

2.4.6 Método do Ponto Interior Primal-Dual . . . . . . . . . . . . . . . 64

2.4.7 Solvers para Otimização Convexa . . . . . . . . . . . . . . . . . . . 64

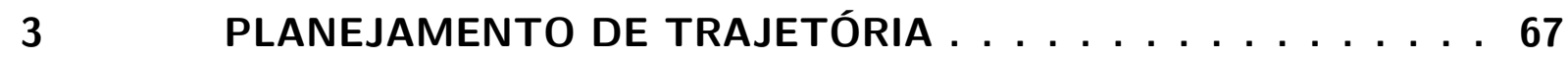

3.1 Definição do Problema . . . . . . . . . . . . . . . . 67

3.1.1 Modelo dinâmico do robô diferencial . . . . . . . . . . . . . . 67

3.1.2 Cinemática do robô em um caminho . . . . . . . . . . . . . . . . . . 69

3.1.3 Dinâmica do robô em um caminho . . . . . . . . . . . . . . . 70

3.1.4 Parametrização geométrica do caminho . . . . . . . . . . . . 71

3.1.5 Problema de otimização Tempo-Energia . . . . . . . . . . . . 71

$3.2 \quad$ Convexificação . . . . . . . . . . . . . . . . . . . . 72

3.2.1 Análise de convexidade . . . . . . . . . . . . . . . . 73

$3.3 \quad$ Linearização Discreta . . . . . . . . . . . . . . . . . . . . . . 75

3.3.1 Discretização das variáveis de otimização . . . . . . . . . . . . . 75

3.3.2 Discretização das equações do problema de otimização . . . . . . . . . . 77

3.4 Formulação como Programação Cônica de Segunda Ordem - SOCP . . . 79

3.5 Resultados Numéricos do Planejamento Ótimo . . . . . . . . . . . . . 82 
3.5.1 Geração de dados . . . . . . . . . . . . . . . . . . . . 82

$3.5 .2 \quad$ Otimização . . . . . . . . . . . . . . . . 85

3.5.3 Fronteira de Pareto . . . . . . . . . . . . . . . . . . . 92

$3.6 \quad$ Sintonia do Coeficiente de Ponderação . . . . . . . . . . . . . . . . . 94

3.6.1 Interpolação da Curva da Fronteira de Pareto . . . . . . . . . . . . . . . 94

3.6.2 Obtenção dos coeficientes $\alpha$ e $\beta \ldots \ldots$. . . . . . . . . . 95

3.6.3 Relação entre $\mu$ e $T_{f} \ldots \ldots \ldots$. . . . . . . . . . . . 96

3.6.4 Cálculo experimental dos coeficientes $\nu$ e $\kappa \ldots \ldots$. . . . . . . . . . 97

3.6.5 Algoritmo de sintonia automática do coeficiente de ponderação . . . . . . . 99

$3.7 \quad$ Conclusão do Capítulo . . . . . . . . . . . . . . . . . . . . 102

4 PlaneJaMento de CAMINHO . . . . . . . . . . 105

4.1 Curvas de Dubins . . . . . . . . . . . . . . . . 105

4.2 Otimização de Trajetória em Curvas Dubins . . . . . . . . . . . . . 106

$4.3 \quad$ Curvas Dubins Suaves . . . . . . . . . . . . . . . . . . . . . . 110

4.3.1 Interpolação por Splines Cúbicas Grampeadas . . . . . . . . . . . . . . . 111

4.3.2 Suavização da Curva Dubins . . . . . . . . . . . . . . . . . 115

$4.4 \quad$ Conclusão do Capítulo . . . . . . . . . . . . . . . . . . . 122

5 RASTREAMENTO DE TRAJETÓRIA . . . . . . . . . . 123

5.1 Definição do Problema . . . . . . . . . . . . . . . . . . . 123

$5.2 \quad$ Controle em Malha Aberta . . . . . . . . . . . . . . . . . . . . 123

5.3 A abordagem "Siga a Cenoura" . . . . . . . . . . . . . . . . . 128

$5.4 \quad$ Controle de Dupla Malha . . . . . . . . . . . . . . . . . . . . . 129

5.4.1 Controle da Cinemática por Feedback Linearization . . . . . . . . . . . . 129

$5.4 .2 \quad$ Controle da Dinâmica . . . . . . . . . . . . . . . . . . . 130

5.4.3 Esquema de Controle . . . . . . . . . . . . . . . . . 131

$5.5 \quad$ Conclusão do Capítulo . . . . . . . . . . . . . . . . . 137

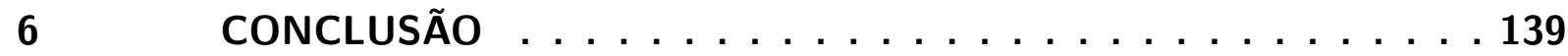

6.1 Publicações decorrentes da Tese . . . . . . . . . . . . . . . . . . . 142

6.2 Trabalhos Futuros . . . . . . . . . . . . . . . . . 142

REFERÊNCIAS . . . . . . . . . . . . . . . . . 145

APÊNDICES 
APÊNDICE B - CÓDIGOS EM MATLAB ${ }^{\circledR} \ldots \ldots \ldots 157$

B.1 Transformação de Coordenadas . . . . . . . . . . . . . 157

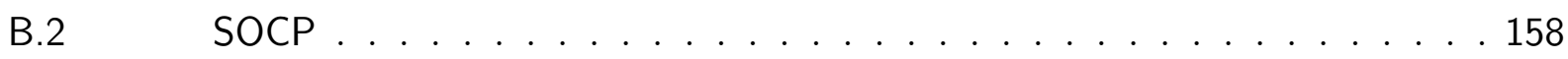

B.3 Clamped Spline . . . . . . . . . . . . . . . . . . . . . . . 160

B.4 Controle Dupla Malha . . . . . . . . . . . . . . . . 162 



\section{INTRODUÇÃO}

A Robótica Móvel tem sido um campo de pesquisa ativa e com um desenvolvimento científico e tecnológico significativo nas últimas três décadas. Este interesse é alimentado principalmente por uma vasta gama de aplicações práticas nas quais os veículos autônomos têm sido utilizados pela sociedade - na exploração de ambientes hostis e inóspitos, no resgate e transporte de vítimas, na assistência a pessoas com mobilidade ou visão reduzida, no monitoramento aéreo de grandes regiões e até no lazer de hobistas. Se utilizado para fins pacíficos, estes veículos autônomos podem trazer melhorias significativas para a qualidade de vida do ser humano e benefícios para a sociedade.

Em aplicações de veículos autônomos em áreas remotas e isoladas - como por exemplo, em ambientes espaciais ou submarinos - a recuperação ou reabastecimento energético é difícil ou muitas vezes impossível. Por este motivo, considerações quanto à autonomia energética são de fundamental importância nos projetos de robôs.

Pela multidisciplinaridade deste campo de pesquisa, a autonomia energética de veículos autônomos pode ser maximizada pela otimização de seus subsistemas em sua etapa de projeto, incluindo a capacidade das baterias, aspectos estruturais de construção, eficiência em motores e drivers. Entretanto, ganhos consideráveis podem ser obtidos com o planejamento eficiente de sua movimentação. Este campo de estudos tem emergido como uma forma alternativa interessante para minimizar o consumo energético de robôs móveis, e ainda não tem sido suficientemente explorado na comunidade científica (LIU; SUN, 2014).

Reconhecendo a importância da preservação dos recursos energéticos, este trabalho tem como principal motivação aproveitar o potencial exploratório nesta área de conhecimento para trazer reflexões e resultados no campo de estudo do planejamento eficiente na movimentação de robôs móveis, contribuindo desta forma para o avanço científico e tecnológico da Robótica Móvel.

\subsection{ESCOPO E PROBLEMA DE PESQUISA}

Os robôs móveis sobre rodas (wheeled mobile robots - WMR) consomem menos energia e se movem mais rapidamente se comparados a outras classes de veículos terrestres, como por exemplo robôs com pernas, com esteiras ou veículos sobre trilhos. Do ponto de vista energético, veículos sobre rodas exigem um menor esforço de controle, devido aos seus mecanismos simples e problemas de estabilidade reduzidos (SIEGWART; NOURBAKHSH, 2004). 
Das várias topologias possíveis para WMRs, a classe de robôs diferenciais possui características específicas que motivaram sua utilização como modelo para este trabalho. Sua ampla utilização como opção em aplicações práticas se deve à simplicidade de seu aspecto construtivo - um chassis com duas rodas motorizadas individualmente - o que leva a um custo reduzido em relação às outras topologias. Em contrapartida, restrições nãoholonômicas conferem a esta topologia desafios extras para o controle de seu movimento. Estes desafios, que tecnologicamente são encarados como obstáculos, são considerados aspectos motivadores no campo da pesquisa científica. Por estes motivos escolheu-se o robô diferencial como modelo para este trabalho.

Dentre os sistemas que compõem um robô móvel está o sistema de controle responsável pelo seu movimento. Das arquiteturas de controle de movimento presentes na literatura, uma das mais utilizadas até os dias de hoje é o Controlador Hierárquico Aninhado, do inglês Nested Hierarchical Controller (NHC), proposto inicialmente por Meystel (1986); uma arquitetura de controle hierárquico deliberativo, cuja adaptação é ilustrada na Figura 1.

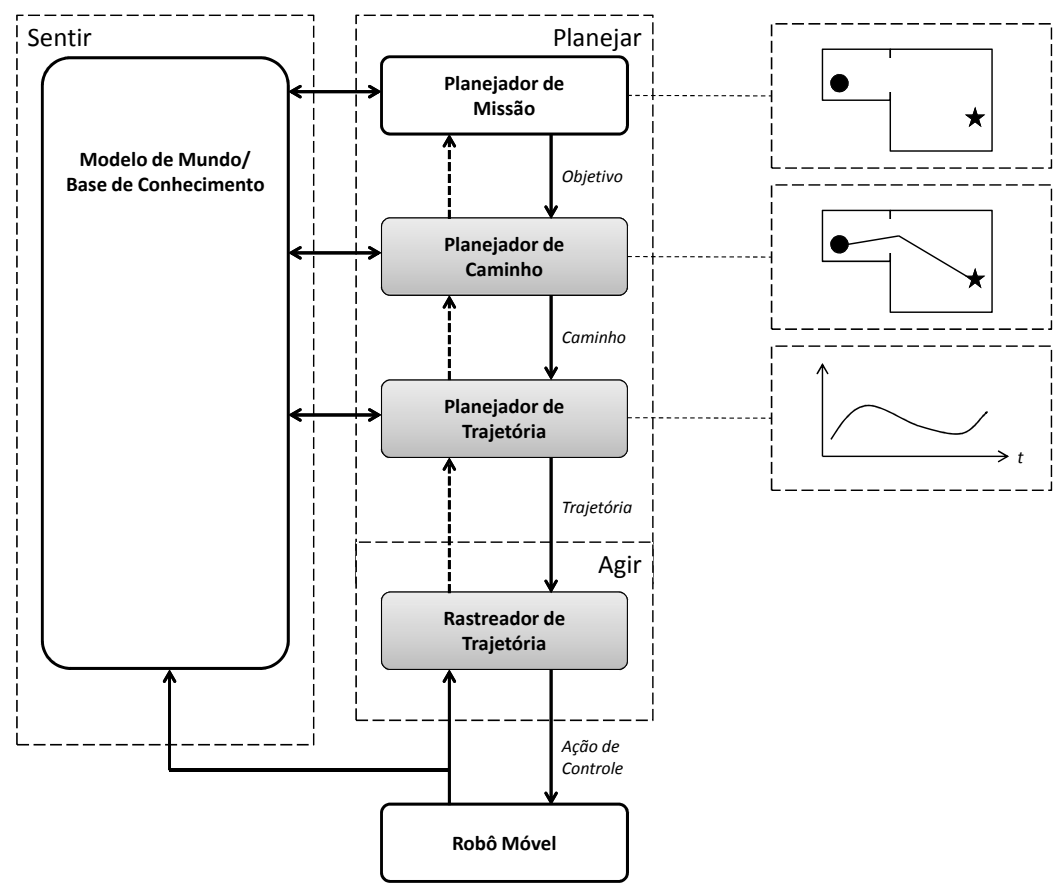

Figura 1 - Diagrama da arquitetura NHC.

Fonte: adaptado de Murphy (2000).

Nesta adaptação da arquitetura NHC, a tarefa de Planejar é decomposta em três níveis hierárquicos de abstração: o Planejador de Missão, o Planejador de Caminho e o Planejador de Trajetória. Descendo de um nível para outro, o planejamento se torna mais específico e detalhado. Obedecendo critérios definidos pelo usuário, o Planejador de Missão tem como tarefa gerar objetivos específicos da missão, mais comumente pontos de controle (ou waypoints) pelos quais o robô deverá passar. 
Como exemplo, o Planejador de Missão deve indicar ao robô para sair da sua estação de recarregamento, ir até um armazém para pegar um palete, levá-lo até uma máquina específica e retornar à estação inicial. Estes objetivos se traduzem geometricamente como pontos no plano por onde o robô deva passar.

Dados os pontos de controle, a tarefa do Planejador de Caminho é de encontrar um caminho possível entre estes pontos, levando em consideração aspectos do ambiente por onde o robô deve percorrer. Em um ambiente estruturado - em que se conhece previamente a posição dos obstáculos - o algoritmo de planejamento de caminho busca, dentre os possíveis caminhos entre os pontos de controle, aquele que melhor atende a uma determinada regra de otimização, como ilustra a Figura 2.

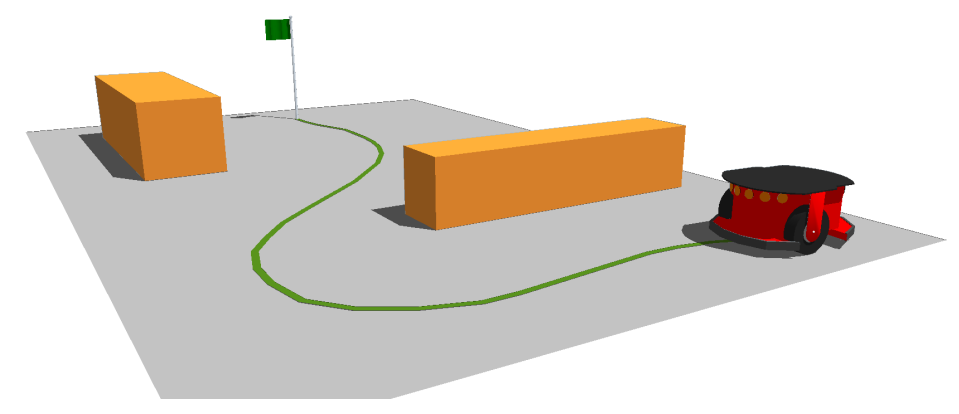

Figura 2 - Um WMR e seu caminho em um ambiente estruturado.

Fonte: o autor.

Dado então o caminho gerado pela etapa anterior, a tarefa específica do Planejador de Trajetória é gerar um perfil de velocidade que deverá ser realizado pelo robô durante o percurso no caminho. Os termos caminho e trajetória, apesar de comumente utilizados como sinônimos, têm seus significados diferentes dentro da literatura em Robótica e serão utilizados neste trabalho. Enquanto o termo caminho se refere ao percurso geométrico específico na qual um robô deva percorrer, o termo trajetória carrega consigo, além dos aspectos geométricos definidos pelo caminho, aspectos temporais definidos por um perfil de velocidade.

Já o papel do Rastreador de Trajetória no contexto da arquitetura NHC é de gerar as ações de controle necessárias para que o robô possa desenvolver a trajetória especificada pelo nível hierárquico superior.

Conforme o robô se move, novas informações são adquiridas por meio dos sensores do robô para atualização do modelo do mundo. Quando necessário, o Planejador de Trajetória corrige o movimento local do robô para controlar as eventuais divergências percebidas; ou o Planejador de Caminho encontra uma melhor solução para evitar colisões com obstáculos. Se ainda assim esta correção não for suficiente, o Planejador de Missão refaz o planejamento da missão, apresentando novos objetivos ao Planejador de Caminho. Este ciclo permite que o robô possa se movimentar em ambientes com obstáculos móveis ou em coordenação com outros robôs, por exemplo. 
Este trabalho atua sobre um recorte específico da arquitetura NHC, focando especificamente nos planejamentos de caminho e de trajetória, e no rastreamento de trajetória para uma classe específica de robôs móveis. Para exemplificar, considere a Figura 3 como ilustração de um problema a ser resolvido.

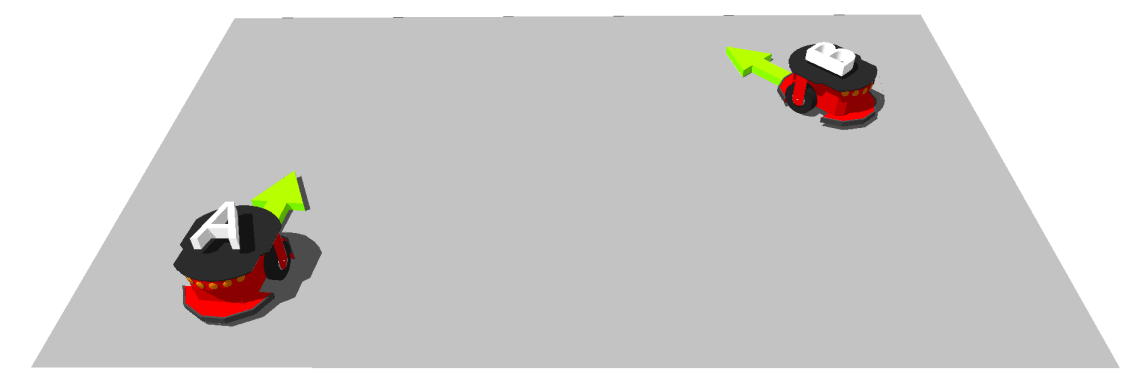

Figura 3 - O problema de movimento entre duas posturas.

Fonte: o autor.

Seja um WMR diferencial que se encontra em um ambiente estruturado e livre de obstáculos. Em um plano, as possíveis configurações de um robô diferencial são descritas pela sua posição (onde o robô se encontra) e sua orientação (para onde o robô está direcionado). Dada então uma postura inicial "A", seja a tarefa definida pelo Planejador de Missão que o robô parta da postura inicial e alcance uma postura final "B".

O escopo deste trabalho é o recorte da arquitetura NHC que, dada esta determinada missão, realiza o planejamento do caminho entre duas posturas específicas; o planejamento do perfil de velocidade a ser desenvolvido pelo robô no caminho dado em função do tempo; e a geração dos sinais de controle tais que o robô realize a trajetória determinada.

Para avaliar a efetividade na conclusão desta tarefa, dois conceitos clássicos são considerados. O primeiro dos conceitos é a eficácia, que expressa o quão corretamente a tarefa é realizada. Otimizar a eficácia no movimento do WMR significa diminuir o erro geométrico entre o caminho determinado e o percurso que é efetivamente realizado pelo robô no plano.

O segundo conceito a ser considerado é a eficiência. Ela expressa a relação entre o resultado alcançado e os recursos empregados - o quão a tarefa foi realizada "da melhor maneira possível". Este conceito depende do contexto que está sendo usado. Uma compra eficiente é aquela na qual a relação entre os benefícios do recurso adquirido e o custo monetário de sua compra é maior possível; um computador eficiente é aquele que consegue realizar uma determinada tarefa computacional em menor tempo possível.

No caso do movimento de um WMR, dois recursos são importantes para serem considerados na avaliação da eficiência: (i) o tempo de percurso e (ii) a energia consumida para a execução da tarefa. Usando o exemplo ilustrativo da Figura 2, a execução mais eficiente da tarefa dada é aquela na qual o robô percorra o caminho dado no menor tempo possível, consumindo a menor energia possível. 
É claro perceber que são dois objetivos inversamente proporcionais. Independentemente do modelo matemático proposto para o robô, percorrer o caminho dado em um menor tempo possível requer um consumo energético maior, tendo em vista as inércias a serem vencidas durante o percurso. Pelo mesmo motivo, porém de outro lado, realizar o caminho consumindo a menor energia possível significa diminuir os esforços de controle e, por consequência, aumentar o tempo total de percurso (STANHOUSE; KITTS; MAS, 2016).

Dentro da perspectiva da arquitetura NHC e considerando as motivações desta pesquisa, o problema de movimento do WMR pode ser decomposto em três sub-problemas: (i) gerar uma geodésica (ou seja, o caminho mais curto entre dois pontos) matematicamente representado como uma curva em $\mathbb{R}^{2}$; (ii) gerar uma trajetória refletida em um perfil de velocidade tal que o robô realize o caminho proposto de forma eficiente; e (iii) dado o perfil de velocidade, gerar um sinal de controle tal que o robô realize o caminho proposto de forma eficaz. Tanto a eficiência relativa aos resultados, quanto a eficácia relativa aos recursos (PEARSON, 2017), irão depender de especificações pré-definidas, como por exemplo a relação entre o custo monetário do tempo e da energia.

\subsection{OBJETIVOS DE PESQUISA}

Então, seja um WMR diferencial presente em um ambiente estruturado e com a missão determinada de partir de uma postura inicial e alcançar uma postura final. Seja também definida uma relação de custo monetário $\Lambda$ entre tempo e energia.

O presente trabalho tem como principal objetivo projetar e implementar computacionalmente um recorte da arquitetura NHC para controlar o movimento de um WMR diferencial de forma eficiente e eficaz.

Para tanto, os três níveis de abstração serão tratados de forma separada. A camada superior, o Planejador de Caminho, tem por objetivo encontrar a geodésica entre as duas posturas que, por ser o menor caminho possível, é também o mais eficiente.

A camada intermediária, o Planejador de Trajetória, é responsável por encontrar um sinal de controle expresso em tensão elétrica e um perfil de velocidade tal que se imponha ao robô o atravessamento do caminho encontrado considerando limites de velocidade e aceleração. Estes sinais devem ser ótimos no sentido de que o tempo final e a energia total consumida durante o percurso sejam os mínimos possíveis dentro de uma relação de custo entre tempo e energia.

Dado o perfil de velocidade pelo Planejador de Trajetória, a camada inferior na arquitetura NHC - o Rastreador de Trajetória - é responsável por encontrar os sinais de controle tais que o robô siga com o menor erro possível o caminho dado. 


\subsection{CONTRIBUIÇÕES DA TESE}

As principais contribuições que este trabalho apresenta são:

- As coordenadas generalizadas que classicamente são usadas no modelo do WMR diferencial presentes na literatura não permitem com que robôs sub-atuados possam ser usados como modelos em problemas de otimização. Esta constatação é observada nos trabalhos de Verscheure et al. (2008), Verscheure et al. (2009), Lipp e Boyd (2014), Reynoso-Mora, Chen e Tomizuka (2016). Esta Tese defende que o fato do robô percorrer um caminho específico pode ser usado para reconfigurar o modelo do robô em um sistema de coordenadas generalizadas alternativas que permita a utilização deste modelo no método que a literatura propõe.

- Com os resultados do Planejador de Trajetória, verificou-se que os objetivos de Tempo Final e Energia Total apresentam uma Fronteira de Pareto com características exponenciais. Esta Tese apresenta um algoritmo inédito de sintonia que tem como finalidade encontrar o melhor dos ótimos (CHEIKH et al., 2010) dentro dos possíveis pontos desta Fronteira de Pareto.

- A solução clássica para se encontrar a geodésica entre duas posturas é apresentada por Dubins (1957), e desde então tem sido usada na literatura (SHKEL; LUMELSKY, 2001; CHO; RYEU, 2006; GIESE, 2012; LUGO-CARDENAS et al., 2014). Entretanto, esta geodésica, conhecida como "curva de Dubins", não é duplamente diferenciável no plano especificamente em dois pontos desta curva. Como consequência, nestes pontos específicos a execução da tarefa de rastreamento torna-se impraticável. Esta Tese oferece uma solução para este problema suavizando as curvas de Dubins de tal forma a minimizar o problema da segunda derivada na curva sem prejudicar consideravelmente sua característica geodésica.

- O Rastreador de Trajetória proposto nesta Tese usa uma modificação da abordagem Siga a Cenoura proposta inicialmente por Rankin (1997) para modelar o problema como um sistema de erros. Um controlador de Linearização por Realimentação (KHALIL, 1996) é proposto para rastrear a trajetória definida pelo Planejador. Apesar desta técnica de controle ser usada na literatura para rastreamento de trajetória, este trabalho reinvindica o ineditismo no sentido do controle ser baseado na abordagem Siga a Cenoura.

\subsection{ORGANIZAÇÃO DO TEXTO}

As variáveis escalares são apresentadas tanto em alfabeto romano quanto em alfabeto grego, enquanto vetores e matrizes são apresentados em negrito. 
A revisão da literatura é apresentada ao leitor no Capítulo 2. Esta revisão permite ao leitor se situar no problema proposto, apresentando os principais trabalhos relacionados com o controle de movimento para robôs móveis, tanto no que se diz respeito ao planejamento de trajetória quanto ao seu rastreamento. O Capítulo também apresenta a teoria que envolve o campo de estudos de Otimização Convexa e o Estado da Arte deste campo para resolução de problemas de planejamento de trajetória, situando o leitor no contexto em que esta Tese pretende defender como contribuições.

A ordem dos capítulos dessa Tese não coincide com a ordem hierárquica dos subsistemas da arquitetura NHC proposta. No Capítulo 3, o problema de otimização tempo-energia é formulado como uma proposta de Planejamento de Trajetória para o robô, dado um caminho genérico. Uma transformação de coordenadas generalizadas é apresentado para que o modelo do WMR seja usado em um problema de Otimização Convexa. Então, o problema é discretizado e formulado como uma Programação Cônica de Segunda Ordem (BOYD; VANDENBERGHE, 2004). Resultados preliminares são apresentados ao leitor no sentido de demonstrar a relação exponencial existente entre os objetivos do problema de otimização. O algoritmo de sintonia é apresentado neste Capítulo e seus resultados discutidos.

Para além de curvas genéricas, a utilização de curva Dubins como caminho a ser usado na etapa de Planejamento de Trajetória é discutido no Capítulo 4. Uma suavização dessa classe de curvas é proposta como alternativa para o Planejamento de Caminho.

O Capítulo 5 apresenta inicialmente os resultados do controle em malha aberta, ou seja, utilizando os sinais de controle ótimo provenientes do Planejador de Trajetória. Em seguida, apresenta um controlador feedback-feedforward que tem como princípio corrigir os sinais de controle ótimo do Planejador com os erros relativos entre a posição do robô e o caminho de referência $\Gamma$ dado. Um controlador por feedback linearization é proposto para rastrear a trajetória baseado em um sistema de erros entre a postura do robô e o caminho de referência. Os resultados dos três controladores são comparados.

Por fim, o Capítulo 6 apresenta as considerações finais da Tese, a análise de suas contribuições e apesenta um encaminhamento para as pesquisas futuras. 



\section{REVISÃO DA LITERATURA}

O presente capítulo apresenta as bases científicas necessárias e suficientes para o desenvolvimento desta Tese. O robô diferencial é contextualizado dentro do campo científico da Robótica Móvel e seu modelo cinemático apresentado e analisado. O estado da arte do controle de movimento e de planejamento de trajetória é revisto e, por fim, o campo de estudo de Otimização Convexa é apresentada ao leitor.

\subsection{CINEMÁTICA DE ROBÔS MÓVEIS}

O estudo da cinemática na robótica móvel é importante para compreender o comportamento do robô tanto para projetar a estrutura ideal para realizar determinada tarefa quanto para seu projeto de controle. Ele herda boa parte do conhecimento científico trazido do campo de estudo dos robôs manipuladores, porém uma diferença chave entre ambos os campos de conhecimento traz um desafio significativo para a robótica móvel: a estimação de posição.

Enquanto para um manipulador, a posição sempre pode ser computada pelos dados dos sensores de forma instantânea pela cinemática inversa, na robótica móvel não existe um meio direto para se medir a posição de um robô de forma instantânea. Deve-se, então, integrar o movimento do robô a cada instante. Somando-se a isto as incertezas devidas a deslizamentos, fica claro que medir a posição exata de um robô móvel é uma tarefa extremamente desafiadora.

O modelo cinemático do robô uniciclo diferencial considera o robô como um corpo rígido sob rodas, operando em um plano horizontal. São consideradas três dimensões que caracterizam o número de graus de liberdade do robô: duas para a sua posição no plano e uma para a orientação do eixo de simetria do robô em relação ao plano. Também considera-se o chassis do robô como seu corpo rígido, ignorando as juntas e os graus de liberdade internos ao robô e também as suas rodas (D'ANDREA-NOVEL; CAMPION; BASTIN, 1993).

\subsubsection{Modelo cinemático do robô uniciclo diferencial}

Como ilustrado na Figura 4, um robô uniciclo diferencial é um veículo autônomo constituído por um chassi rígido dotado de uma roda louca e duas rodas fixas iguais de raio $r$, montadas paralelamente em um eixo imaginário $Y_{P}$, a uma distância $B$ entre elas, e cada uma das duas rodas acionadas por um motor elétrico independente (AICARDI et al., 1995). 


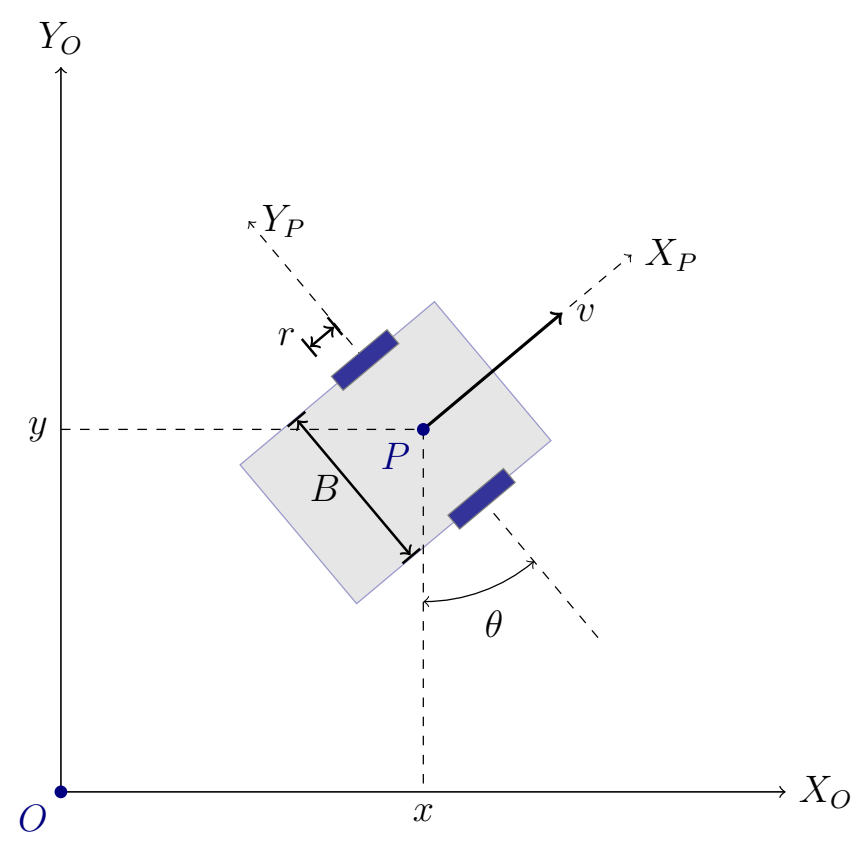

Figura 4 - O robô diferencial e seus sistemas referenciais.

Fonte: o autor.

Sejam um sistema de coordenadas cartesianas global $\mathcal{F}_{O}:\left\{X_{O}, Y_{O}\right\}$ a partir de uma origem inercial $O$, e um sistema de coordenadas do corpo do veículo a partir do ponto médio entre as rodas $P$ dado por $\mathcal{F}_{P}:\left\{X_{P}, Y_{P}\right\}$.

Define-se posição do ponto $P$ no sistema global de referência $\mathcal{F}_{O}$ o vetor $\mathbf{p} \in \mathbb{R}^{2}$ da forma

$$
\mathbf{p}=\left[\begin{array}{ll}
x & y
\end{array}\right]^{T}
$$

onde $x$ e $y$ são as coordenadas cartesianas do ponto $P$ nos eixos $X_{O}$ e $Y_{O}$, respectivamente; e define-se postura do robô como sendo as suas coordenadas generalizadas em $\mathcal{F}_{O}$, representadas pelo vetor $\boldsymbol{\xi} \in \mathbb{R}^{3}$ como

$$
\boldsymbol{\xi}=\left[\begin{array}{ll}
\mathbf{p} & \theta
\end{array}\right]^{T}=\left[\begin{array}{lll}
x & y & \theta
\end{array}\right]^{T}
$$

onde $\theta \in[0,2 \pi]$ é a diferença angular entre ambos os sistemas de referência.

O mapeamento entre os sistemas de coordenadas $\mathcal{F}_{O}$ e $\mathcal{F}_{P}$ é dado pela matriz de rotação ortogonal:

$$
\boldsymbol{\mathcal { R }}(\theta)=\left[\begin{array}{ccc}
\cos \theta & \operatorname{sen} \theta & 0 \\
-\operatorname{sen} \theta & \cos \theta & 0 \\
0 & 0 & 1
\end{array}\right]
$$

Esta matriz pode ser usada para mapear o movimento entre os dois sistemas de coordenadas pela operação $\dot{\boldsymbol{\xi}}_{\boldsymbol{P}}=\boldsymbol{\mathcal { R }}(\theta) \dot{\boldsymbol{\xi}}$. 
Parametrizando o vetor de postura $\boldsymbol{\xi}(t): \mathbb{R} \rightarrow \mathbb{R}^{3}$ no tempo, o modelo cinemático do robô é representado pela equação diferencial matricial

$$
\dot{\boldsymbol{\xi}}(t)=\left[\begin{array}{c}
\dot{x}(t) \\
\dot{y}(t) \\
\dot{\theta}(t)
\end{array}\right]=\left[\begin{array}{cc}
\cos \theta(t) & 0 \\
\operatorname{sen} \theta(t) & 0 \\
0 & 1
\end{array}\right]\left[\begin{array}{c}
v(t) \\
\omega(t)
\end{array}\right],
$$

na qual $v(t)$ é a velocidade linear do robô na direção de $X_{P}$, e $\omega(t)$ é a velocidade angular do robô no sentido anti-horário.

\subsubsection{Análise das Restrições Cinemáticas do Robô Uniciclo Diferencial}

As restrições cinemáticas do chassis do robô são dadas pela união das restrições cinemáticas de cada roda que o compõe. O robô uniciclo diferencial é constituído por duas rodas fixas convencionais alinhadas no eixo $Y_{R}$ e uma roda louca (castor wheel) posicionada no eixo $X_{R}$ numa distância do ponto $P$ não nula de tal forma que forme um plano paralelo ao solo. Assume-se que o plano de cada roda sempre se mantém vertical e que existe apenas um ponto de contato com o plano do solo; isto é, as rodas se movem sempre na condição de rolamento sobre o eixo vertical através do seu ponto de contato com o solo.

Apesar da cinemática da roda louca ser consideravelmente complexa, estas rodas não impõem qualquer restrição real na cinemática do chassis do robô. As restrições são então dadas apenas pelas duas rodas convencionais fixas $\left(N_{f}=2\right)$. O modelo genérico desta roda é ilustrada na Figura 5, onde $A$ indica a posição da roda relativa ao sistema de coordenadas do chassis $\mathcal{F}_{P}:\left\{X_{P}, Y_{P}\right\}$, expressa em coordenadas polares dadas por $l$ e $\alpha$. O ângulo do plano da roda em relação ao chassis é dado por $\beta$. A roda de raio $r$ pode girar livremente em seu eixo de rotação com velocidade $\dot{\varphi}$.

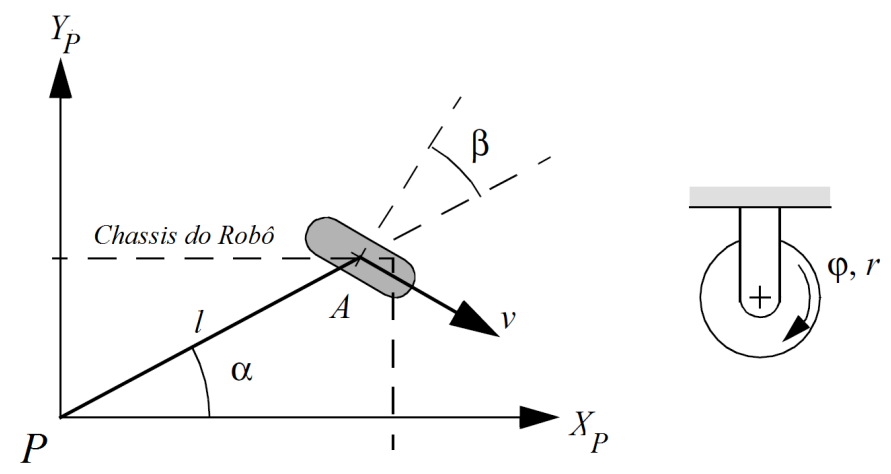

Figura 5 - Uma roda fixa convencional e seus parâmetros.

Fonte: adaptado de Siegwart e Nourbakhsh (2004)

Siegwart e Nourbakhsh (2004) apresentam dois tipos de restrições para as rodas do robô uniciclo com rodas fixa convencionais. A primeira restrição é relacionada com o conceito de contato de rolagem - que a roda deve rolar quando o movimento estiver numa 
direção apropriada, e é dada pela equação

$$
\boldsymbol{J}_{1}\left(\beta_{s}\right) \mathcal{R}(\theta) \dot{\boldsymbol{\xi}}=\boldsymbol{J}_{2} \Omega,
$$

onde $\boldsymbol{J}_{2}$ é uma matriz diagonal constante contendo os valores dos raios para todas as rodas convencionais em sua diagonal, $\Omega$ o vetor contendo as velocidades angulares das rodas e $\boldsymbol{J}_{\mathbf{1}}\left(\beta_{s}\right)$ denota a matriz com projeções para todas as rodas de seus movimentos na direção dos seus planos. Esta matriz contém as projeções para as rodas fixas $\boldsymbol{J}_{\mathbf{1}} \boldsymbol{f}$ e para as direcionáveis $\boldsymbol{J}_{1 s}\left(\beta_{s}\right)$, da forma

$$
\boldsymbol{J}_{\mathbf{1}}\left(\beta_{s}\right)=\left[\begin{array}{c}
\boldsymbol{J}_{1 f} \\
\boldsymbol{J}_{1 s}\left(\beta_{s}\right)
\end{array}\right] .
$$

A restrição de deslizamento reforça o conceito de não existir deslizamento ortogonal ao plano da roda, e é dada pela equação

$$
\boldsymbol{C}_{\mathbf{1}}\left(\beta_{s}\right) \boldsymbol{\mathcal { R }}(\theta) \dot{\boldsymbol{\xi}}=0
$$

Da mesma forma, $\boldsymbol{C}_{\mathbf{1}}\left(\beta_{s}\right)$ carrega as projeções das rodas na direção perpendicular aos seus planos, tanto para as rodas fixas $\boldsymbol{C}_{\boldsymbol{1} \boldsymbol{f}}$ quanto para as direcionáveis $\boldsymbol{C}_{\mathbf{1}}\left(\beta_{\boldsymbol{s}}\right)$, e pode ser escrito como

$$
\boldsymbol{C}_{1}\left(\beta_{s}\right)=\left[\begin{array}{c}
\boldsymbol{C}_{1 f} \\
\boldsymbol{C}_{1 s}\left(\beta_{s}\right)
\end{array}\right] .
$$

As equações 2.5 e 2.7 podem ser combinadas em uma única expressão matricial da forma

$$
\left[\begin{array}{c}
\boldsymbol{J}_{\mathbf{1}}\left(\beta_{s}\right) \\
\boldsymbol{C}_{\mathbf{1}}\left(\beta_{s}\right)
\end{array}\right] \boldsymbol{\mathcal { R }}(\theta) \dot{\boldsymbol{\xi}}=\left[\begin{array}{c}
\boldsymbol{J}_{\mathbf{2}} \boldsymbol{\Omega} \\
0
\end{array}\right] .
$$

Considerando o robô diferencial apresentado na Figura 4, este se movimenta na direção do eixo $X_{P}$. A roda louca não impõe nenhuma restrição de movimento ao robô, sendo portanto, ignorada para esta análise. As duas rodas convencionais são fixas e, portanto,

$$
\begin{aligned}
& \boldsymbol{J}_{\mathbf{1}}\left(\beta_{s}\right)=\boldsymbol{J}_{\mathbf{1 f}}=\left[\begin{array}{lll}
\cos (\alpha+\beta) & \operatorname{sen}(\alpha+\beta) & l \operatorname{sen} \beta
\end{array}\right] \\
& \boldsymbol{C}_{\mathbf{1}}\left(\beta_{s}\right)=\boldsymbol{C}_{\mathbf{1} \boldsymbol{f}}=\left[\begin{array}{lll}
\operatorname{sen}(\alpha+\beta) & -\cos (\alpha+\beta) & l \cos \beta
\end{array}\right] .
\end{aligned}
$$

Como ambas as rodas são paralelas, ambas contribuem com a mesma restrição de deslizamento. Na geometria do robô proposta, para a roda direita tem-se $\alpha=-\pi / 2$, $\beta=\pi$, e para a roda esquerda, $\alpha=\pi / 2, \beta=0$. A distância de ambas as rodas ao centro do chassis é $l=B / 2$. Com estas considerações, calculando as Equações 2.5 e 2.7 têm-se as três restrições de movimento dadas pelas equações:

$$
\begin{aligned}
\dot{x} \cos \theta-\dot{y} \operatorname{sen} \theta+(b / 2) \dot{\theta} & =r \dot{\varphi}_{r}, \\
\dot{x} \cos \theta-\dot{y} \operatorname{sen} \theta-(b / 2) \dot{\theta} & =r \dot{\varphi}_{l}, \\
\dot{x} \operatorname{sen} \theta-\dot{y} \cos \theta & =0 .
\end{aligned}
$$


As equações 2.12 e 2.13 impõem as restrições de rolagem das rodas esquerda e direita, respectivamente, enquanto a equação 2.14 restringe o deslizamento do chassis do robô na direção do eixo $Y_{R}$.

Estas são consideradas restrições cinemáticas não-holonômicas. Em robótica móvel, este termo refere-se especificamente a restrições cinemáticas do chassis, quando estas são relacionadas com termos diferenciais. Um robô é dito não-holonômico quando este possui um ou mais restrições cinemáticas não-holonômicas. A Figura 6 apresenta as relações geométricas entre as velocidades do robô que implicam na relação trigonométrica

$$
\operatorname{tg} \theta=\frac{\operatorname{sen} \theta}{\cos \theta}=\frac{\dot{y}}{\dot{x}},
$$

equivalente à equação 2.14 .

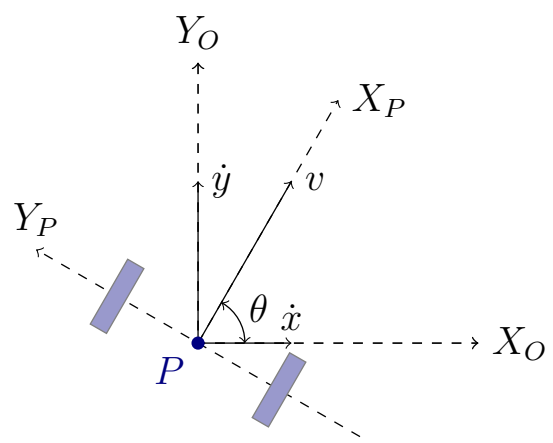

Figura 6 - Relação geométrica entre os vetores de velocidade do robô.

Fonte: o autor.

\subsubsection{Análise da Manobrabilidade do Robô Uniciclo Diferencial}

A manobrabilidade cinemática de um chassis robótico é sua habilidade de se mover em um ambiente, cuja restrição básica é a que satisfaz a restrição de não-deslizamento. Então, sua análise está diretamente ligada à Equação 2.7.

Os graus de liberdade global sob o qual um robô pode se mover, chamado de graus de manobrabilidade $\delta_{M}$, pode ser definido por

$$
\delta_{M}=\delta_{m}+\delta_{s}
$$

onde $\delta_{m}=3-\operatorname{posto}\left[\boldsymbol{C}_{\mathbf{1}}\left(\beta_{s}\right)\right]$ são os graus de mobilidade do robô, e $\delta_{s}=\operatorname{posto}\left[\boldsymbol{C}_{\mathbf{1 s}}\left(\beta_{s}\right)\right]$ são os graus de direcionabilidade do robô (SICILIANO; KHATIB, 2008). Como o robô proposto neste trabalho não possui rodas direcionáveis - apenas duas rodas fixas - sua configuração define $\delta_{s}=0$ e $\delta_{m}=2$ e, portanto, a manobrabilidade deste robô tem a dimensionalidade de $\delta_{M}=2$.

Em robótica móvel, o termo manobrabilidade se refere à dimensão dos graus de liberdade do controle de seu movimento. De fato, pode-se empiricamente verificar que existem apenas as velocidades de cada uma das rodas fixas $\dot{\varphi}_{r}$ e $\dot{\varphi}_{l}$ do robô diferencial como forma de atuação. 


\subsubsection{Análise do Espaço de Trabalho do Robô Uniciclo Diferencial}

O robô móvel está situado em um ambiente. Portanto, é importante saber como o robô pode se mover entre várias configurações, quais os tipos de caminhos que ele pode seguir e, além disso, quais as possíveis trajetórias ele pode realizar no espaço de configuração. Para tanto, define-se o espaço de velocidade admissível, que descreve os componentes independentes do movimento do robô que este pode controlar. No caso do uniciclo, este espaço pode ser representado por dois eixos - um representando a velocidade instantânea do robô na direção de $X_{R}$, e outro representando a mudança de orientação $\dot{\theta}$ do uniciclo.

Os graus de liberdade do espaço de trabalho (DOF- degrees of freedom) determinam a habilidade do robô de alcançar várias configurações (poses), e os graus de liberdade diferenciáveis (DDOF- differentiable degrees of freedom) determinam sua habilidade para alcançar os caminhos. A dimensionalidade de DDOF será sempre igual aos graus de mobilidade $\delta_{m}$.

Existe uma relação de desigualdade em relação ao espaço de trabalho dado por $D D O F \leq \delta_{M} \leq D O F$ (SIEGWART; NOURBAKHSH, 2004). Um robô é dito holonômico se e somente se $D D O F=D O F$. Como o robô proposto neste trabalho possui $D D O F=2$, e seu ambiente $D O F=3$, ele é um robô não-holonômico.

\subsection{CONTROLE DE MOVIMENTO DE ROBÔS MÓVEIS}

Convencionalmente, os problemas de controle de movimento de veículos autônomos encontrados na literatura podem ser classificados em três categorias básicas (SICILIANO; KHATIB, 2008; TZAFESTAS, 2014):

- Estabilização de Postura - Pose Stabilisation

O objetivo deste problema de controle é estabilizar o vetor de postura $\boldsymbol{\xi}=\left[\begin{array}{lll}x & y & \theta\end{array}\right]^{T}$ do robô em uma determinada postura de referência $\boldsymbol{\xi}_{c}$, como mostra a subfigura 7 a. Um exemplo real correspondente a este problema é o estacionamento de um veiculo em uma determinada vaga de garagem.

- Seguimento de Caminho - Path Following

O objetivo do controle é ter o ponto $P$ fixo no robô seguindo a curva $\Gamma \in \mathcal{F}_{O}$ quando o robô se move com uma velocidade fixa $v_{0} \geq 0$. A variável que se deve estabilizar em zero é, então, a distância $d$ entre o ponto $P$ e o ponto $C$ mais próximo de $\Gamma$, como ilustrado na subfigura 7b. Este tipo de problema tipicamente corresponde a dirigir em uma rodovia enquanto se tenta manter constante a distância entre o veículo e o lado da rodovia. 
- Rastreamento de Trajetória - Trajectory Tracking

Este problema difere do último no sentido de que a velocidade longitudinal do veículo não é pré-fixada. O problema de rastreamento supõe que a curva geométrica $\Gamma$ é complementada com a velocidade da referência parametrizada com a variável tempo $t$. Isto leva a definir a trajetória $t \longmapsto\left(x_{r e f}(t), y_{r e f}(t)\right) \in \mathcal{F}_{O}$. Então, o objetivo é estabilizar o vetor de erro linear de posição $\boldsymbol{e}=\left(x_{r e f}(t)-x(t), y_{r e f}(t)-y(t)\right)$ a zero, como mostra a subfigura $7 \mathrm{c}$.

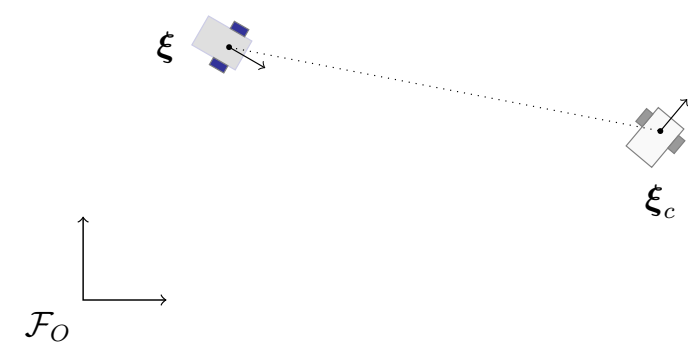

(a) Estabilização de Postura,

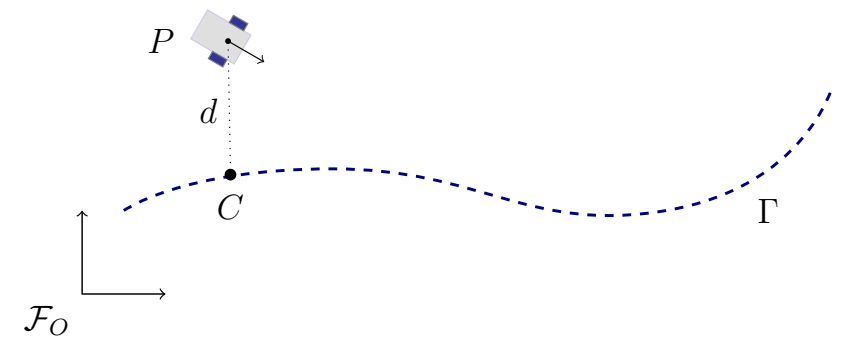

(b) Seguimento de Caminho,

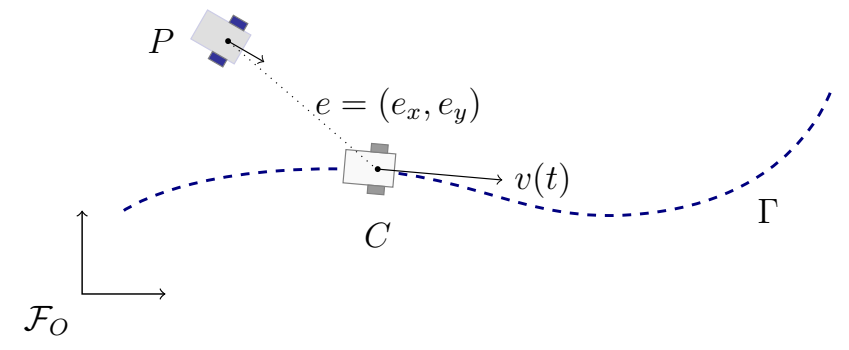

(c) Rastreamento de Trajetória.

Figura 7 - Problemas clássicos de controle de movimento de robôs móveis encontrados na literatura.

Fonte: adaptado de Luca, Oriolo e Samson (1998).

O trabalho pioneiro de Dubins (1957) propõe resolver o problema de estabilização de postura. Ele demonstra que, para um veículo com restrições não-holonômicas em um espaço bidimensional, qualquer geodésica (i.e. o caminho mais curto) entre duas posturas é sempre constituído de três curvas, que podem ser arcos para a esquerda (L) e direita 
(R), ou um seguimento de reta $(\mathrm{S})$. O caminho de tempo ótimo será sempre pelo menos um dos seis tipos: RSR, RSL, LSR, LSL, RLR e LRL.

Apesar da proposta de Dubins se demonstrar eficaz para planejamento de caminho, o problema de estabilização de postura para sistemas não-holonômicos não pode ser resolvido por uma lei de controle realimentado invariante no tempo (BROCKETT, 1983).

Nos anos 90, Murray e Sastry (1991) apresentaram uma conversão das equações cinemáticas de robôs não-holonômicos em uma forma nilpotente que denominaram forma encadeada (chained form). Os autores usaram uma mudança das variáveis de estado e de controle e mostraram que é possível, utilizando esta forma, encontrar caminhos para conduzir o robô entre posturas arbitrárias.

Esta forma de representação foi usada no trabalho pioneiro de Samson (1995) para desenvolver controladores realimentados como solução dos problemas de estabilização de postura. A partir destes trabalhos pioneiros, duas abordagens principais têm sido propostos na literatura: controle variante no tempo e realimentação descontínua (KOLMANOVSKY; MCCLAMROCH, 1995; ZHEN-YING; CHAO-LI, 2011; SHANG; XIE, 2014; ALGER; VOTION; QIAN, 2014).

A problemática do rastreamento de trajetória para sistemas holonômicos é bem compreendida, e soluções satisfatórias podem ser encontradas em livros de texto na área de controle não linear (SLOTINE; LI, 1991; ISIDORI, 1995; KHALIL, 1996). Porém, para sistemas não-holonômicos, o problema ainda é foco de intensa pesquisa na área.

Tzafestas (2014) apresenta uma série de métodos de controle para robôs móveis sob rodas, baseados em uma ampla pesquisa na literatura. Para movimento em ambientes controlados (ou seja, baseado em regras determinísticas e conhecidas), e com parâmetros cinemáticos e dinâmicos do robô precisamente conhecidos, é suficiente empregar algum tipo de controle baseado em modelo.

Nesta categoria, o autor apresenta algumas técnicas de projeto de controladores por linearização baseadas no critério de estabilidade de Lyapunov (KANAYAMA et al., 1991; AICARDI et al., 1995); utilizando linearização por realimentação de estados (state feedback linearization) de sistemas dinâmicos afins (YUN; YAMAMOTO, 1992; YANG et al., 2004); assim como algumas técnicas de controle seguindo a metodologia de variedades invariantes $^{1}$ (REYHANOGLU, 1995; TAYEBI; TADJINE; RACHID, 2001; WATANABE et al., 2010)

Porém, se o modelo do robô apresenta incertezas e/ou ele se movimenta em ambientes não controlados, o algoritmo de controle deve ser mais sofisticado, envolvendo algum tipo de inteligência.

1 Do inglês invariant manifolds. Apesar do termo em português parecer antitético, é desta forma que é tratado dentro da literatura em controle na língua portuguesa. 
Uma das metodologias básicas para tratar de incertezas do sistema é a metodologia de controle adaptativo, que emprega um algoritmo para identificar os parâmetros variáveis em tempo real. Dentre as abordagens mais usadas estão o controle adaptativo por modelo de referência (ASHOORIRAD et al., 2006) e a técnica de controle adaptativo por backstepping (POURBOGHRAT; KARLSSON, 2002; HUANG; TSAI, 2008).

Outra metodologia empregada é o controle robusto, que requer um conhecimento a priori dos limites das variações dos parâmetros, pois quanto mais preciso o conhecimento destes limites, melhor é a robustez alcançada por esses controladores (DIXON et al., 2000; LI; YE, 2014). Neste mesmo sentido, Chwa (2004) apresenta uma resolução utilizando controle por modos deslizantes.

Por fim, Tzafestas (2014) apresenta algumas metodologias de IA, como a resolução do problema de controle de movimento para robôs móveis sob rodas. Sistemas de controle por lógica fuzzy e por redes neurais são aproximadores universais, ou seja, eles podem aproximar (ou mapear) qualquer função não linear com certa precisão. Controladores para movimento de robôs baseados nestas abordagens são apresentados pelo autor (RIGATOS; TZAFESTAS; TZAFESTAS, 2000; DAS; KAR, 2006)

O controle para o problema de seguimento de caminho (path following) parte do pressuposto de que a velocidade de avanço do veículo é mantida constante, enquanto o controlador age sobre a orientação do veículo para guiá-lo no caminho.

Observa-se que os controladores de seguimento de caminho realizam uma convergência mais suave, pois são menos propensos à saturação quando comparados com os controladores de rastreamento de trajetória (LUCA; ORIOLO; SAMSON, 1998). De fato, estes dois problemas foram historicamente abordados de forma separada. Encarnação e Pascoal (2002) apresentam uma abordagem de controle para robôs combinando os problemas de seguimento de caminho e de rastreamento de trajetória. Esta abordagem tem sido aperfeiçoada por outras pesquisas (AGUIAR; HESPANHA, 2007; XIANG et al., 2011; ALESSANDRETTI; AGUIAR; JONES, 2013).

\subsubsection{Controlabilidade para o Problema de Rastreamento de Trajetória}

Ainda que o modelo do robô diferencial seja sub-atuado, ele é controlável em seu espaço de configuração (LUCA; ORIOLO; SAMSON, 1998), ou seja, dadas duas configurações arbitrárias, sempre existe uma trajetória cinemática admíssivel (com suas entradas de velocidade associadas) que transfere o robô entre uma configuração e outra.

Porém, a controlabilidade do modelo uniciclo é intrinsecamente não-linear. Então, para estudá-la, é necessário usar ferramentas da teoria de controle não-linear. Esta subseção apresenta apenas a análise da controlabilidade para o problema de Rastreamento de Trajetória. 
Seja o vetor de postura do robô dado por $\boldsymbol{\xi}(t)=\left[\begin{array}{lll}x(t) & y(t) & \theta(t)\end{array}\right]^{T}$, e o vetor que define a postura de referência para movimento $\boldsymbol{\xi}_{c}(t)=\left[\begin{array}{lll}x_{c}(t) & y_{c}(t) & \theta_{c}(t)\end{array}\right]^{T}$. A postura de referência define também as velocidades lineares e angulares de referência, respectivamente $v_{c}$ e $\omega_{c}$.

Definindo o erro de rastreamento como $\tilde{\boldsymbol{\xi}}=\boldsymbol{\xi}-\boldsymbol{\xi}_{\boldsymbol{c}}$, e as variações de velocidade como $\tilde{v}=v-v_{c}$ e $\tilde{\omega}=\omega-\omega_{c}$, a linearização tangencial (SLOTINE; LI, 1991) do sistema definido pela Equação 2.4 é dada por

$$
\dot{\tilde{\xi}}=\left[\begin{array}{ccc}
0 & 0 & -v_{c} \operatorname{sen} \theta_{c} \\
0 & 0 & v_{c} \cos \theta_{c} \\
0 & 0 & 0
\end{array}\right] \tilde{\boldsymbol{\xi}}+\left[\begin{array}{cc}
\cos \theta_{c} & 0 \\
\operatorname{sen} \theta_{c} & 0 \\
0 & 1
\end{array}\right]\left[\begin{array}{c}
\tilde{v} \\
\tilde{\omega}
\end{array}\right]
$$

que é da forma $\dot{\tilde{\boldsymbol{\xi}}}=\mathbf{A}(t) \tilde{\boldsymbol{\xi}}+\mathbf{B}(t)\left[\begin{array}{ll}\tilde{\boldsymbol{v}} & \tilde{\omega}\end{array}\right]^{T}$. Como o sistema linearizado é variante no tempo, uma condição necessária e suficiente para a sua controlabilidade é que seu Gramiano não seja singular.

Luca, Oriolo e Vendittelli (2001) apresentam uma forma simplificada deste sistema, que pode ser construída mapeando-o no sistema de coordenadas do robô $\mathcal{F}_{P}$, através da transformação $\tilde{\boldsymbol{\xi}}_{\boldsymbol{P}}=\boldsymbol{\mathcal { R }}(\theta) \tilde{\boldsymbol{\xi}}$. Assim, a equação 2.17 pode ser reescrita como

$$
\dot{\tilde{\boldsymbol{\xi}}}_{\boldsymbol{P}}=\left[\begin{array}{ccc}
0 & \omega_{c} & 0 \\
-\omega_{c} & 0 & v_{c} \\
0 & 0 & 0
\end{array}\right] \tilde{\boldsymbol{\xi}}_{\boldsymbol{P}}+\left[\begin{array}{ll}
1 & 0 \\
0 & 0 \\
0 & 1
\end{array}\right]\left[\begin{array}{c}
\tilde{v} \\
\tilde{\omega}
\end{array}\right]
$$

Somente quando $v_{c}$ e $\omega_{c}$ são constantes (i.e. a trajetória é uma linha reta ou uma circunferência, com velocidade linear constante), o sistema linear da Equação 2.18 é invariante no tempo.

Então, seja a matriz de controlabilidade dada por

$$
\boldsymbol{C}=\left[\begin{array}{lll}
\mathbf{B} & \mathbf{A B} & \mathbf{A}^{2} \mathbf{B}
\end{array}\right]=\left[\begin{array}{cccccc}
1 & 0 & 0 & 0 & -\omega_{c}^{2} & v_{d} \omega_{c} \\
0 & 0 & -\omega_{c} & v_{c} & 0 & 0 \\
0 & 1 & 0 & 0 & 0 & 0
\end{array}\right]
$$

A condição de controlabilidade se dá por Posto $[\mathcal{C}]=3 \Longleftrightarrow\left[\begin{array}{ll}v_{c} & \omega_{c}\end{array}\right]^{T} \neq\left[\begin{array}{ll}0 & 0\end{array}\right]^{T}$. Portanto, conclui-se que o sistema cinemático dado pela Equação 2.4 pode ser localmente estabilizado por realimentação linear sobre uma trajetória que consiste em caminhos lineares ou circulares, executadas com velocidades constantes. 


\subsection{PLANEJAMENTO DE TRAJETÓRIA}

O planejamento ótimo de trajetórias pode ampliar o desempenho de um robô consideravelmente, particularmente quando as principais preocupações em seu movimento têm relação com a sua eficiência ou seu consumo energético. No sentido de se obter uma trajetória ótima sobre limitações de movimento, não só a cinemática mas também a dinâmica do robô deve ser explicitada na fase de planejamento. As interações entre geometria, cinemática e dinâmica aumentam a complexidade do problema consideravelmente em relação a abordagens puramente cinemáticas.

Seja um robô dado em um determinado ambiente. Sejam também definidos uma configuração inicial e final. O problema de Planejamento de Trajetória consiste em gerar entradas de referência para o sistema de controle de movimento do robô (Rastreador de Trajetória), tal que realize o movimento entre a configuração inicial e final definidas, desviando-se de obstáculos nele presentes. Este problema lida com duas classes básicas de requisitos:

\section{- Requisitos Geométricos}

Do ponto de vista cinemático, considerando a dimensionalidade da postura do robô (duas coordenadas cartesianas e uma de orientação), a trajetória deve ser planejada em um espaço tridimensional com o propósito de evitar colisões com obstáculos. Estes obstáculos podem ser tanto objetos presentes no ambiente de trabalho quanto paredes, portas e corredores pelos quais o robô deverá passar. O problema dado por estes requisitos é encontrar caminhos admissiveis entre a posição inicial e final do robô - path planning, ilustrada de forma simplificada na Figura 8.

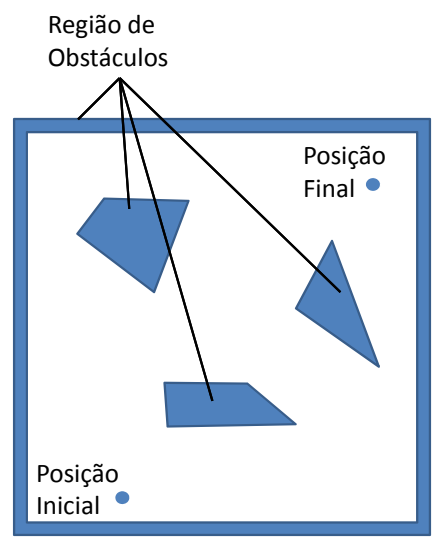

(a)

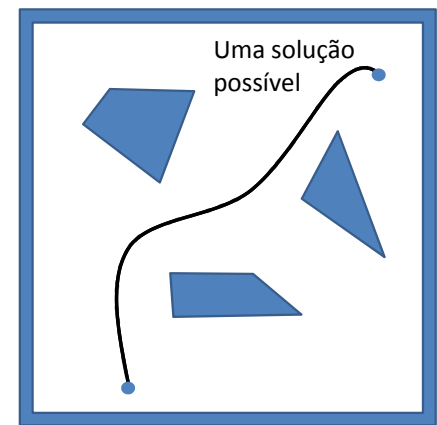

(b)

Figura 8 - Uma ilustração simplificada do problema de planejamento de caminho. (a) Os obstáculos, a posição inicial, e a posição final especificados como entradas. (b) Um algoritmo de planejamento resolve um caminho livre de colisões.

Fonte: adaptado de LaValle (2006). 
- Requisitos Dinâmicos

Do ponto de vista do modelo dinâmico, trajetórias possíveis do robô devem satisfazer requisitos relacionados com a saturação do atuador, limites máximos de velocidades e acelerações, condições de fricção das rodas com pontos de contato, etc. Por exemplo, acelerações longitudinais e angulares devem permanecer abaixo de um dado limite superior para certificar um rolamento puro das rodas, sem deslizamento com o solo, pois forças centrífugas dependem da velocidade linear e da curvatura local do caminho.

Em geral, mais de uma trajetória satisfaz ambos os requisitos (geométricos e dinâmicos). Então, é necessário realizar um procedimento para escolher, dentre todas as trajetórias admissíveis, a trajetória ótima que minimize uma determinada função de custo.

Canny et al. (1988) apresentam pela primeira vez na literatura o termo Restrições Cinemáticas-Dinâmicas, que sintetizam simultaneamente as restrições cinemáticas e dinâmicas, usando um espaço de estado aumentado $\mathcal{Q}$ em que o estado $\mathbf{q} \in \mathcal{Q}$ é definido como $\mathbf{q}=(\boldsymbol{\xi}, \dot{\boldsymbol{\xi}})$, composto por sua configuração aumentada das coordenadas de velocidade (LAVALLE; Kuffner, J.J., 2001).

Existem duas abordagens principais para o planejamento de trajetórias sobre restrições cinodinâmicas. A primeira abordagem busca resolver o problema de planejamento, tratando com ambos os requisitos de forma concomitante, através das restrições cinodinâmicas. Esta abordagem demonstra ser probabilisticamente completa (i.e., se a solução existe, ela pode ser encontrada com probabilidade 1 depois de longa execução) (LAVALLE; Kuffner, J.J., 2001), e tem sido usada como ferramenta para o planejamento de trajetórias (CANNY; REGE; REIF, 1991; DONALD et al., 1993; PHAM; CARON; NAKAMURA, 2013).

Porém, a abordagem cinodinâmica resulta em um problema com a dimensionalidade dobrada. Por conta disso, algoritmos de planejamento em espaço de estados cinodinâmicos tornam-se impraticáveis do ponto de vista computacional (PHAM; CARON; NAKAMURA, 2013).

Egerstedt (2000) propõe resolver os requisitos geométricos e dinâmicos conjuntamente num problema de programação quadrática. Para tanto, o autor usa splines suaves (smoothing splines) como restrições geométricas; e funções base da forma $g(t)=\mathbf{C}^{T} e^{\mathbf{A} t} \mathbf{b}$, que é a resposta de um sistema representado por variáveis de estado, como restrições dinâmicas. Como função custo, o autor usa a integral quadrática do sinal de entrada.

Usando o Teorema da Dualidade de Lagrange, o autor consegue resolver o problema com resultados interessantes. Porém, o sistema representado por variáveis de estado deve ser controlável e observável, o que não resolve o problema do robô uniciclo diferencial como mostrado na subseção 2.2.1. 
Outra abordagem para planejamento de trajetórias consiste em decompor o problema em dois sub-problemas:

1. Encontrar um caminho livre de colisões no espaço de configuração do robô para satisfazer os requisitos geométricos e;

2. Encontrar uma parametrização temporal ótima deste caminho, levando-se em consideração alguma função de custo.

Existem na literatura alguns métodos para resolver o sub-problema de planejamento de caminho, e as três abordagens mais usadas para tal - road-map, decomposição celular e campos potenciais - podem ser encontrados em LaValle (2006).

O sub-problema de se encontrar um conjunto válido de perfis de velocidade, dado um caminho livre de colisões, tem sido resolvido desde a metade da década de 80 via reparametrização do caminho por uma variável que represente seu comprimento normalizado. A próxima sub-seção apresenta alguns trabalhos relevantes.

\subsubsection{Planejamento ótimo de trajetória}

O trabalho pioneiro de Bobrow, Dubowsky e Gibson (1985) apresenta um algoritmo que calcula os torques de um manipulador para que ele se mova em um dado caminho no menor tempo possível, sujeito a restrições de torque. Os autores propõem que a solução de tempo mínimo seja dada escolhendo os perfis com as maiores velocidades possíveis, respeitando as restrições, controlados por uma curva de chaveamento. Esta solução leva a um controle bang-bang, com elevados valores de arranque (jerk).

Pfeiffer e Johanni (1987) propõem obter a solução do tempo mínimo usando as propriedades geométricas do conjunto transformado das equações dinâmicas. Usando o princípio de máximo de Pontryagin, perfis ótimos e suaves de velocidade podem ser obtidos por shooting methods (SHILLER, 1996; CONSTANTINESCU; CROFT, 2000).

Ainda que estas abordagens apresentassem resultados satisfatórios e importantes para a literatura, suas formulações não-lineares não garantem a otimalidade global.

Verscheure et al. (2008) (ver também (VERSCHEURE et al., 2009)) apresentam uma importante transformação que permite resolver o problema de tempo mínimo e/ou energia mínima para o movimento de um manipulador robótico de seis graus de liberdade (6-DOF) através de um caminho pré-definido, usando as recentes ferramentas de otimização convexa (apresentada na seção 2.4). Algumas aplicações que reforçam esta abordagem podem ser encontradas na literatura (ARDESHIRI et al., 2011; DEBROUWERE et al., 2013; ZHANG; LI; GAO, 2013). 
O trabalho de Lipp e Boyd (2014) expande a abordagem de Verscheure para além de robôs manipuladores. Os autores propõem uma generalização para veículos cuja configuração $\boldsymbol{\xi} \in \mathbb{R}^{p}$ é controlada por atuadores acionados por sinais $\mathbf{u} \in \mathbb{R}^{r}$ e regidos pela dinâmica na forma

$$
\mathbf{R}(\boldsymbol{\xi}) \mathbf{u}=\mathbf{M}(\boldsymbol{\xi}) \ddot{\boldsymbol{\xi}}+\mathbf{C}(\boldsymbol{\xi}, \dot{\boldsymbol{\xi}}) \dot{\boldsymbol{\xi}}+\mathbf{d}(\boldsymbol{\xi})
$$

onde $\mathbf{R}: \mathbb{R}^{p} \rightarrow \mathbb{R}^{p \times r}$ é a matriz de controle, $\mathbf{M}: \mathbb{R}^{p} \rightarrow \mathbb{R}^{p \times p}$ é uma matriz simétrica positiva definida que representa as inércias, $\mathbf{C}: \mathbb{R}^{2 p} \rightarrow \mathbb{R}^{p \times p}$ é a matriz dos efeitos centrífugos e $\mathbf{d}: \mathbb{R}^{p} \rightarrow \mathbb{R}^{p}$ apresentam as forças independentes da configuração.

Os autores apresentam soluções para uma série de modelos como veículos espaciais, veículos sobre rodas e veículos aéreos. Porém, todos os modelos apresentados tem a forma $p \leq r$. Desta forma, a equação matricial (2.20) possui equações suficientes e necessárias para servirem de restrição de igualdade em um problema de otimização.

Algumas inconsistências matemáticas podem ser verificadas nesta formulação da dinâmica robótica em um caminho (ver Apêndice A). Recentemente, Reynoso-Mora, Chen e Tomizuka (2016) perceberam esta inconsistência e reformularam o modelo dinâmico nãolinear (equação 2.20) considerando as fricções de viscosidade e de Couloumb de maneira mais consistente.

\subsection{OTIMIZAÇÃO CONVEXA}

Um problema de otimização é aquele no qual se deseja encontrar $\mathbf{x} \in \mathbb{R}^{n}$ que minimize (ou maximize) uma dada função $f(\mathbf{x}): \mathbb{R}^{n} \rightarrow \mathbb{R}$. De maneira geral, encontrar um ótimo global de uma função não é uma tarefa simples. Porém, para uma classe especial de problemas de otimização denominado problemas de otimização convexa, em muitos casos pode-se encontrar eficientemente a solução global.

Aqui, o termo "eficiente" tem conotações práticas e teóricas: significa que é possível resolver muitos problemas práticos em um tempo razoável, sendo que este tempo depende apenas polinomialmente do tamanho do problema.

Esta seção apresenta uma revisão sobre o tema, baseado no livro de Boyd e Vandenberghe (2004), e com a notação utilizada pelo livro.

\subsubsection{Conjuntos Convexos}

Inicialmente, segue a definição de conjunto convexo:

Definição 2.1. Um conjunto $C$ é convexo se, para todo $\mathbf{x}_{1}, \mathbf{x}_{2} \in C$ e $\theta \in \mathbb{R}, 0 \leq \theta \leq 1$,

$$
\theta \mathbf{x}_{1}+\left(1-\theta \mathbf{x}_{2}\right) \in C
$$


Intuitivamente podemos afirmar que se para cada dois elementos em $C$ traçarmos um segmento de reta, todos os pontos deste segmento de reta (denominada combinação convexa de $\mathbf{x}_{1}$ e $\mathbf{x}_{2}$ ) também pertencem a $C$.

A Figura 9 apresenta dois exemplos: o polígono da subfigura 9a é um exemplo de conjunto convexo; já o conjunto ilustrado na subfigura 9a não é convexo, pois o segmento de linha entre os dois pontos mostrados na figura não pertencem ao conjunto.

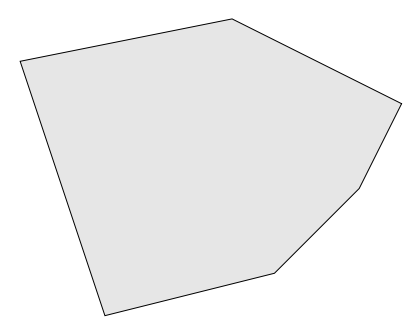

(a)

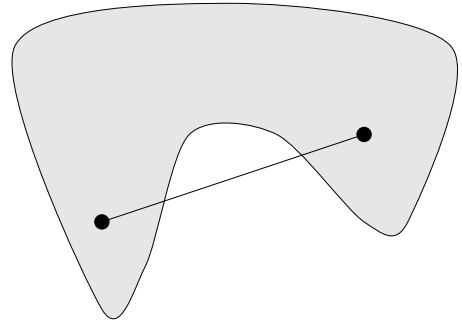

(b)

Figura 9 - Exemplos simples de convexidade de conjuntos.

Fonte: adaptado de Boyd e Vandenberghe (2004).

\subsubsection{Cones Convexos}

Um conjunto $C$ é dito cone se para todo $\mathbf{x} \in C$ e $\theta \geq 0$ tem-se $\theta \mathbf{x} \in C$. Diz-se que $C$ é um cone convexo se ele é convexo e é também um cone, isto é, para todo $\mathbf{x}_{1}, \mathbf{x}_{2} \in C \mathrm{e}$ $\theta_{1}, \theta_{2} \geq 0$, tem-se

$$
\theta \mathbf{x}_{1}+\left(1-\theta \mathbf{x}_{2}\right) \in C
$$

Pontos dessa forma podem ser descritos geometricamente como formando uma fatia de pizza bidimensional com vértice na origem e com seus lados passando através de $\mathbf{x}_{1}$ e $\mathbf{x}_{2}$, como ilustra a Figura 10

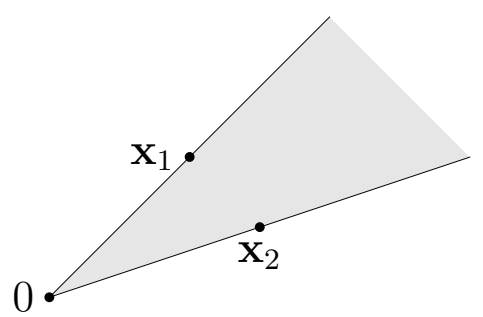

Figura 10 - Exemplo simples de cone convexo. O vértice do cone (que corresponde a $\theta_{1}=\theta_{2}=0$ está em $O$. Seus lados (que correspondem a $\theta_{1}=0$ ou $\theta_{2}=0$ ) passam pelos pontos $\mathbf{x}_{1}$ e $\mathbf{x}_{2}$.

Fonte: adaptado de Boyd e Vandenberghe (2004). 


\subsubsection{Funções Convexas}

Um elemento central em otimização convexa é a noção de função convexa:

Definição 2.2. Uma função $f: \mathbb{R}^{n} \rightarrow \mathbb{R}$ é convexa se seu domínio dom $(f)$ é um conjunto convexo e se, para todo $\mathbf{x}, \mathbf{y} \in \operatorname{dom}(f)$, e $\theta \in \mathbb{R}, 0 \leq \theta \leq 1$,

$$
f(\theta \mathbf{x}+(1-\theta) \mathbf{y}) \leq \theta f(\mathbf{x})+(1-\theta) f(\mathbf{y})
$$

Geometricamente, esta desigualdade significa que o segmento de reta entre $(\mathbf{x}, f(\mathbf{x}))$ e $(\mathbf{y}, f(\mathbf{y}))$, que é a corda de $\mathbf{x}$ para $\mathbf{y}$, está acima do gráfico de (f), como ilustra a Figura 11

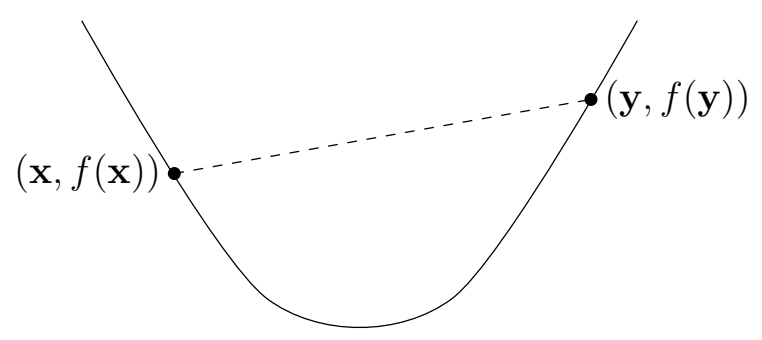

Figura 11 - Gráfico de uma função convexa. A corda (o segmento de reta tracejado) entre quaisquer dois pontos no gráfico se encontra sobre o gráfico da função.

Fonte: adaptado de Boyd e Vandenberghe (2004).

Uma função $f$ é dita estritamente convexa se a inegualdade na equação (2.23) for estrita, mesmo que $\mathbf{x} \neq \mathbf{y}$ e $0<\theta<1$. Diz-se que $f$ é côncava se $-f$ é convexa e estritamente côncava se $-f$ é estritamente convexa.

\subsubsection{Condição de primeira ordem para a convexidade}

Supõe-se que uma função $f: \mathbb{R}^{n} \rightarrow \mathbb{R}$ é diferenciável (i.e., o gradiente $\nabla_{x} f(\mathbf{x})$ ) existe em todos os pontos $\mathbf{x} \in \operatorname{dom}(f)$. Então, $f$ é convexo se e somente se $\operatorname{dom}(f)$ é um conjunto convexo, e para todo $\mathbf{x}, \mathbf{y} \in \operatorname{dom}(f)$,

$$
f(\mathbf{y}) \geq f(\mathbf{x})+\nabla_{x} f(\mathbf{x})^{T}(\mathbf{y}-\mathbf{x})
$$

A função afim de $\mathbf{y}$ dada por $f(\mathbf{x})+\nabla_{x} f(\mathbf{x})^{T}(\mathbf{y}-\mathbf{x})$ é uma aproximação de Taylor de primeira ordem para a função $f$ no ponto $\mathbf{x}$, e graficamente é a reta tangente à função $f$ no ponto $\mathbf{x}$ como ilustra a Figura 12 .

A aproximação de Taylor de primeira ordem é, de fato, um estimador de mínimo global de uma função $f$ convexa. A desigualdade dada pela Equação (2.24) mostra que, de uma informação local sobre uma função convexa (o valor e sua derivada em um ponto), pode-se obter uma informação global (o estimador de mínimo).

Similarmente à definição de convexidade, $f$ será estritamente convexa se a Equação (2.24) recair numa desigualdade estrita; côncava se a desigualdade é reversa, e estritamente côncava se a desigualdade reversa é restrita. 


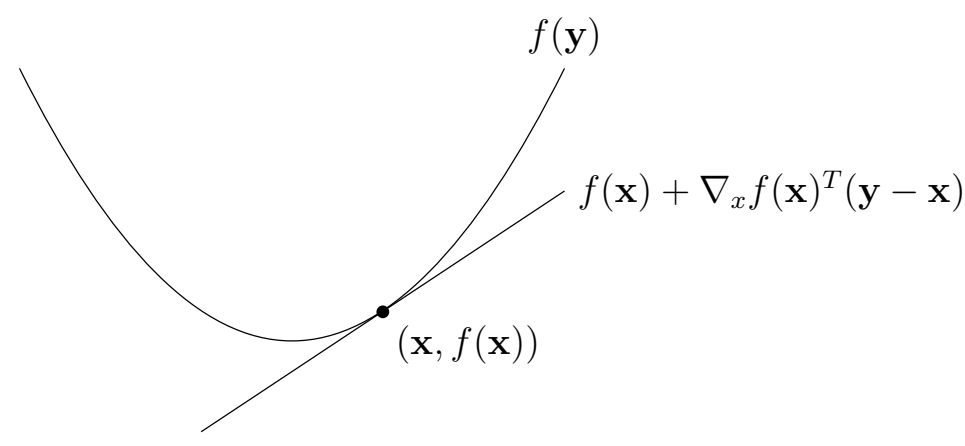

Figura 12 - Ilustração da condição de primeira ordem para a convexidade. Fonte: adaptado de Boyd e Vandenberghe (2004).

\subsubsection{Condição de segunda ordem para a convexidade}

Supõe-se que uma função $f: \mathbb{R}^{n} \rightarrow \mathbb{R}$ é duplamente diferenciável (i.e., a Hessiana $\nabla_{x}^{2} f(\mathbf{x})$ é definida para todos os pontos $\left.\mathbf{x} \in \operatorname{dom}(f)\right)$. Então, $f$ é convexo se e somente se $\operatorname{dom}(f)$ é um conjunto convexo, e sua Hessiana é semidefinida positiva - ou seja, para todo $\mathbf{x} \in \operatorname{dom}(f)$,

$$
\nabla_{x}^{2} f(\mathbf{x}) \succeq 0
$$

\subsubsection{Problemas de Otimização Convexa}

Baseadas nas Definições 2.1 e 2.2, formalmente um problema de otimização convexa é um problema de otimização da forma

$$
\begin{array}{ll}
\text { minimize : } & f_{0}(\mathbf{x})=\mathcal{J}(\mathbf{x}) \\
\text { sujeito a }: & f_{i}(\mathbf{x}) \leq 0, \quad i=1, \ldots, m \\
& h_{j}(\mathbf{x})=0, \quad j=1, \ldots, p,
\end{array}
$$

onde a função objetivo $\mathcal{J}(\mathbf{x})$ e as restrições de desigualdade $f_{i}(\mathbf{x})$ são funções convexas, e as restrições de igualdade $h_{j}(\mathbf{x})=\mathbf{a}_{j}^{T} \mathbf{x}-\mathbf{b}_{j}$ devem ser funções afins de $\mathbf{x}$.

A região possível de um problema de otimização convexa é o conjunto convexo $\mathcal{D}=\left\{\mathbf{x} \mid f_{i}(\mathbf{x}) \leq 0, i=1, \ldots, m, \mathbf{a}_{j}^{T} \mathbf{x}-\mathbf{b}_{j}, j=1, \ldots, p\right\}$. O valor ótimo de um problema de otimização $\mathcal{J}^{*}$ é o mínimo valor possível da função objetivo na região possível, i.e., $\mathcal{J}^{*}=\inf \left\{f_{0}(\mathbf{x}) \mid \mathbf{x} \in \mathcal{D}\right\}$. Diz-se que um ponto $\mathbf{x}^{*}$ é um ponto ótimo se $f_{0}\left(\mathbf{x}^{*}\right)=\mathcal{J}^{*}$.

\subsubsection{Otimalidade global em problemas de Otimização Convexa}

Intuitivamente, um ponto possível é dito ótimo local se não existem pontos possíveis em sua vizinhança que possuam seus valores objetivos menores. Similarmente, um ponto possível é dito ótimo global se não existe nenhum ponto possível que possua seu valor objetivo menor. De maneira formal: 
Definição 2.3. Um ponto $\mathbf{x}$ é ótimo local se $\mathbf{x} \in \mathcal{D}$ e se existe algum $R>0$ tal que todo $\mathbf{z} \in \mathcal{D},\|\mathbf{x}-\mathbf{z}\|_{2} \leq R$, satisfaça $f_{0}(\mathbf{x}) \leq f_{0}(\mathbf{z})$

Definição 2.4. Um ponto $\mathbf{x}$ é ótimo global se $\mathbf{x} \in \mathcal{D}$ e para todo $\mathbf{z} \in \mathcal{D}, f_{0}(\mathbf{x}) \leq f_{0}(\mathbf{z})$

Uma propriedade de problemas de otimização convexa é de fundamental importância nessa Tese.

Teorema 2.1. Para um problema de otimização convexa, todo ponto ótimo local é também um ótimo global.

Demonstração. Supõe-se que $\mathbf{x} \in \mathcal{D}$ é um ótimo local que não seja um ótimo global, ou seja, existe um ponto $\mathbf{y} \in \mathcal{D}$ tal que $f_{0}(\mathbf{x})>f_{0}(\mathbf{y})$. Pela Definição 2.3, não existe ponto $\mathbf{z} \in \mathcal{D}$ tal que $\|\mathbf{x}-\mathbf{z}\|_{2} \leq R$ e $f_{0}(\mathbf{x}) \leq f_{0}(\mathbf{z})$. Suponha-se que se escolha o ponto

$$
\mathbf{z}=\theta \mathbf{y}+(1-\theta) \mathbf{x}, \quad \theta=\frac{R}{2\|\mathbf{x}-\mathbf{y}\|_{2}} .
$$

Então

$$
\begin{aligned}
\|\mathbf{x}-\mathbf{y}\|_{2} & =\left\|\mathbf{x}-\left(\frac{R}{2\|\mathbf{x}-\mathbf{y}\|_{2}} y+\left(1-\frac{R}{2\|\mathbf{x}-\mathbf{y}\|_{2}}\right) \mathbf{x}\right)\right\|_{2} \\
& =\left\|\frac{R}{2\|\mathbf{x}-\mathbf{y}\|_{2}}(\mathbf{x}-\mathbf{y})\right\|_{2} \\
& =R / 2 \leq R .
\end{aligned}
$$

Além disso, pela convexidade de $f_{0}$, tem-se

$$
f_{0}(\mathbf{z})=f_{0}(\theta \mathbf{y}+(1-\theta) \mathbf{x}) \leq \theta f_{0}(\mathbf{y})+(1-\theta) f_{0}(\mathbf{x})<f_{0}(\mathbf{x}) .
$$

Como o conjunto possível é convexo e $\mathbf{x}, \mathbf{y} \in \mathcal{D}, \mathbf{z}=\theta \mathbf{y}+(1-\theta) \mathbf{x}$ também é possível. Então, $\mathbf{z} \in \mathcal{D}$ é um ponto possível com $\|\mathbf{x}-\mathbf{z}\|_{2} \leq R$ e $f_{0}(\mathbf{z}) \leq f_{0}(\mathbf{x})$, o que contradiz a suposição inicial. Portanto, $\mathbf{x}$ é um ótimo global.

\subsubsection{Casos especiais de Otimização Convexa}

Por vezes é conveniente considerar problemas de otimização como casos especiais de problemas de otimização convexa (CP). Para estes casos especiais existem algoritmos com relativa eficiência que podem resolver um grande número de problemas.

- Programação Linear (LP) é um problema de otimização convexa no qual a função objetivo e as restrições de igualdade são funções afins. Formalmente, é um problema da forma

$$
\begin{aligned}
\operatorname{minimize}: & \mathbf{c}^{T} \mathbf{x}+d \\
\text { sujeito a : } & \mathbf{G x} \preceq \mathbf{h} \\
& \mathbf{A x}=\mathbf{b}
\end{aligned}
$$


onde $\mathbf{x} \in \mathbb{R}^{n}$ é a variável de otimização, $\mathbf{c} \in \mathbb{R}^{n}, d \in \mathbb{R}, \mathbf{G} \in \mathbb{R}^{m \times n}, \mathbf{h} \in \mathbb{R}^{m}$, $\mathbf{A} \in \mathbb{R}^{p \times n}, \mathbf{b} \in \mathbb{R}^{p}$ são definidos pelo problema, e o símbolo $\preceq$ denota desigualdade por elemento.

- Programação Quadrática (QP) é um problema de otimização convexa na qual a função objetivo é uma função quadrática, e as restrições de igualdade são funções afins. São da forma

$$
\begin{aligned}
\operatorname{minimize}: & 1 / 2 \mathbf{x}^{T} \mathbf{P} \mathbf{x}+\mathbf{c}^{T} \mathbf{x}+d \\
\text { sujeito a }: & \mathbf{G x} \preceq \mathbf{h} \\
& \mathbf{A x}=\mathbf{b},
\end{aligned}
$$

onde $\mathbf{x} \in \mathbb{R}^{n}$ é a variável de otimização, $\mathbf{c} \in \mathbb{R}^{n}, d \in \mathbb{R}, \mathbf{G} \in \mathbb{R}^{m \times n}, \mathbf{h} \in \mathbb{R}^{m}$, $\mathbf{A} \in \mathbb{R}^{p \times n}, \mathbf{b} \in \mathbb{R}^{p}$ são definidos pelo problema, assim como $\mathbf{P} \in \mathbb{S}_{+}^{n}$ que é uma matriz simétrica semidefinida positiva.

- Programação Quadrática com Restrições Quadráticas (QCQP) é um problema de otimização convexa no qual tanto a função objetivo quanto as restrições de igualdade são funções quadráticas, ou seja,

$$
\begin{aligned}
\text { minimize : } & 1 / 2 \mathbf{x}^{T} \mathbf{P} \mathbf{x}+\mathbf{c}^{T} \mathbf{x}+d \\
\text { sujeito a : } & 1 / 2 \mathbf{x}^{T} \mathbf{Q} \mathbf{x}+\mathbf{r}_{i}^{T} \mathbf{x}+\mathbf{s}_{i} \leq 0 \quad i=1, \ldots, m \\
& \mathbf{A} \mathbf{x}=\mathbf{b},
\end{aligned}
$$

onde $\mathbf{x} \in \mathbb{R}^{n}$ é a variável de otimização, $\mathbf{c} \in \mathbb{R}^{n}, d \in \mathbb{R}, \mathbf{A} \in \mathbb{R}^{p \times n}, \mathbf{b} \in \mathbb{R}^{p}, \mathbf{P} \in \mathbb{S}_{+}^{n}$, e também tem-se $\mathbf{Q} \in \mathbb{S}_{+}^{n}, \mathbf{r}_{i} \in \mathbb{R}^{n}, \mathbf{s}_{i} \in \mathbb{R}$, para $i=1, \ldots, m$.

- Programação Cônica de Segunda Ordem (SOCP) é um problema de otimização convexa no qual a função objetivo é uma função linear, e as desigualdades são generalizadas em um cone quadrático convexo, da forma

$$
\begin{aligned}
\text { minimize : } & \mathbf{f}^{T} \mathbf{x} \\
\text { sujeito a : } & \left\|\mathbf{A}_{i} \mathbf{x}+\mathbf{b}_{i}\right\|_{2} \leq \mathbf{c}_{i}^{T} \mathbf{x}+d_{i} \quad i=1, \ldots, m \\
& \mathbf{F x}=\mathbf{g},
\end{aligned}
$$

onde $\mathbf{x} \in \mathbb{R}^{n}$ é a variável de otimização, $\mathbf{c}, \mathbf{f} \in \mathbb{R}^{n}, d \in \mathbb{R}, \mathbf{A}_{i} \in \mathbb{R}^{n_{i} \times n}, \mathbf{F} \in \mathbb{R}^{p \times n}$, $\mathbf{b}, \mathbf{g} \in \mathbb{R}^{p}$. As restrições de igualdade da forma $\|\mathbf{A x}+\mathbf{b}\|_{2} \leq \mathbf{c}^{T} \mathbf{x}+d$ são chamadas "restrições de segunda ordem", pois a função afim de $\left(\mathbf{A} \mathbf{x}+\mathbf{b}, \mathbf{c}^{T} \mathbf{x}+d\right)$ recai dentro de um cone de segunda ordem $K \in \mathbb{R}^{k+1}$.

Esta forma também pode ser expressa na forma com desigualdade generalizada:

$$
\begin{aligned}
\text { minimize : } & \mathbf{f}^{T} \mathbf{x} \\
\text { sujeito a : } & -\left(\mathbf{A}_{i} \mathbf{x}+\mathbf{b}_{i},\left(\mathbf{c}_{i}^{T} \mathbf{x}+d_{i}\right) \preceq_{K_{i}} 0 \quad i=1, \ldots, m\right. \\
& \mathbf{F} \mathbf{x}=\mathbf{g},
\end{aligned}
$$


em que $K_{i}=\left\{(\mathbf{y}, t) \in \mathbb{R}^{k_{i}+1} \quad \mid\|\mathbf{y}\|_{2} \leq t\right\}$

- Programação Semidefinida (SDP) é um problema de otimização convexa cuja restrição de igualdade recai em um cone positivo semidefinido $K \in \mathbb{S}_{+}^{k}$, no espaço de matrizes reais simétricas positivas $n \times n$, e tem a forma

$$
\begin{aligned}
\operatorname{minimize}: & \mathbf{f}^{T} \mathbf{x} \\
\text { sujeito a : } & \mathbf{x}_{1} \mathbf{F}_{1}+\cdots+\mathbf{x}_{n} \mathbf{F}_{n}+\mathbf{G} \preceq 0 \\
& \mathbf{A x}=\mathbf{b},
\end{aligned}
$$

onde $\mathbf{G}, \mathbf{F}_{1}, \ldots, \mathbf{F}_{n} \in \mathbb{S}^{k}, \mathbf{A} \in \mathbb{R}^{p \times n}, \mathbf{f}, \mathbf{b} \in \mathbb{R}^{p}$. A desigualdade no SDP é uma desigualdade matricial linear (LMI). Se todas as matrizes $\mathbf{G}, \mathbf{F}_{1}, \ldots, \mathbf{F}_{n}$ são diagonais, então o LMI é equivalente a um conjunto de $n$ desigualdades lineares, e a SDP se reduz a uma LP.

Os problemas de otimização convexa seguem a hierarquia

$$
\mathrm{LP} \subset \mathrm{QP} \subset \mathrm{QCQP} \subset \mathrm{SOCP} \subset \mathrm{SDP} \subset \mathrm{CP}
$$

\subsubsection{Método do Ponto Interior}

De forma genérica, problemas de otimização convexa com restrições de desigualdade são da forma

$$
\begin{aligned}
\operatorname{minimize}: & f_{0}(\mathbf{x})=\mathcal{J}(\mathbf{x}) \\
\text { sujeito a }: & f_{i}(\mathbf{x}) \leq 0, \quad i=1, \ldots, m \\
& \mathbf{A x}=\mathbf{b},
\end{aligned}
$$

onde $f_{0}, \ldots, f_{m}: \mathbb{R}^{n} \rightarrow \mathbb{R}$ são funções convexas e duplamente diferenciáveis e $\mathbf{A} \in \mathbb{R}^{p \times n}$ $\operatorname{com} \operatorname{Posto}(\mathbf{A})=p<n$. Se o problema tem solução, existe um ponto ótimo $\mathbf{x}^{*}$ que resulta um ponto ótimo $\mathcal{J}^{*}=f_{0}\left(\mathbf{x}^{*}\right)$. Se o problema é estritamente factível, i.e., existe $\mathbf{x} \in \mathcal{D}$ tal que $\mathbf{A} \mathbf{x}=\mathbf{b}$ e $f_{i}(\mathbf{x})<0, i=1, \ldots, m$, então as condições de Slater (SLATER, 1959) são alcançadas e existem duais ótimos $\boldsymbol{\lambda}^{*} \in \mathbb{R}^{m}, \boldsymbol{\nu}^{*} \in \mathbb{R}^{p}$ que, juntamente com $\mathbf{x}^{*}$, satisfazem as condições de Karush-Kuhn-Tucker - KKT (KIRK, 1970):

$$
\begin{aligned}
\mathbf{A} \mathbf{x}^{*}=\mathbf{b}, \quad f_{i}\left(\mathbf{x}^{*}\right) & \leq 0, \quad i=1, \ldots, m \\
\boldsymbol{\lambda}^{*} & \succeq 0, \\
\nabla f_{0}\left(\mathbf{x}^{*}\right)+\sum_{i=1}^{m} \boldsymbol{\lambda}_{i}^{*} \nabla f_{i}\left(\mathbf{x}^{*}\right)+\mathbf{A}^{T} \boldsymbol{\nu}^{*} & =0, \\
\boldsymbol{\lambda}_{i}^{*} f_{i}\left(\mathbf{x}^{*}\right) & =0, \quad i=1, \ldots, m .
\end{aligned}
$$


O problema (2.33) pode ser reformulado implicitando a restrição na função objetivo:

$$
\begin{aligned}
& \text { minimize : } f_{0}(\mathbf{x})+\sum_{i=1}^{m} I_{-}\left(f_{i}(\mathbf{x})\right) \\
& \text { sujeito a : } \mathbf{A x}=\mathbf{b},
\end{aligned}
$$

onde $I_{-}: \mathbb{R} \rightarrow \mathbb{R}$ é a função indicadora para números reais não-positivos,

$$
I_{-}(u)= \begin{cases}0 & u \leq 0 \\ \infty & u>0\end{cases}
$$

A função $I_{-}$pode ser aproximada pela função $\hat{I}_{-}:-\mathbb{R}_{++} \rightarrow \mathbb{R}, \hat{I}_{-}=-(1 / t) \log (-u)$, onde $t>0$ é um parâmetro que define a precisão da aproximação. A Figura 13 ilustra a função $I_{-}$, e a aproximação $\hat{I}_{-}$para alguns valores de $t$. O incremento do parâmetro $t$ melhora a aproximação entre as duas funções.

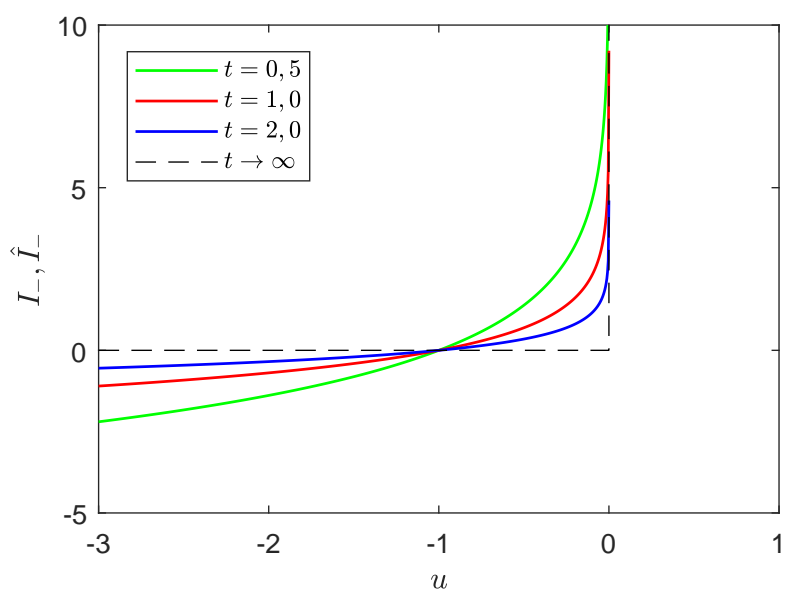

Figura 13 - A função de barreira logarítmica. A linha pontilhada representa a função $I_{-}$, e as curvas em linhas sólidas representam $\hat{I}_{-}$, para $t=0,5 ; 1 ; 2$.

Fonte: adaptado de Boyd e Vandenberghe (2004).

Substituindo $I_{-}$por $\hat{I}_{-}$, o problema (2.38) é equivalente a

$$
\begin{aligned}
& \text { minimize : } t f_{0}(\mathbf{x})+\phi(\mathbf{x}) \\
& \text { sujeito a : } \mathbf{A x}=\mathbf{b},
\end{aligned}
$$

que pode ser resolvido pelo Método de Newton (FLETCHER, 1987), onde a função

$$
\phi(\mathbf{x})=-\sum_{i=1}^{m} \log \left(-f_{i}(\mathbf{x})\right)
$$

com dom $\phi=\left\{\mathbf{x} \in \mathbb{R}^{N} \mid f_{i}(\mathbf{x})<0, i=1, \ldots, m\right\}$ é chamada de barreira logarítmica do problema (2.33). 
Seja $\mathbf{x}^{*}(t)$ a solução do problema (2.40) para cada $t>0$. O caminho central associado ao problema (2.33) é definido como o conjunto de pontos ótimos $\mathbf{x}^{*}(t), t>0$, chamados de pontos centrais. Os pontos centrais são estritamente factíveis, i.e.

$$
\mathbf{A} \mathbf{x}^{*}=\mathbf{b}, \quad f_{i}\left(\mathbf{x}^{*}\right) \leq 0, \quad i=1, \ldots, m
$$

satisfazendo a condição KKT (2.34). Além disso, existe um $\hat{\boldsymbol{\nu}} \in \mathbb{R}^{P}$ tal que

$$
\begin{aligned}
0 & =t \nabla f_{0}\left(\mathbf{x}^{*}(t)\right)+\nabla \phi\left(\mathbf{x}^{*}(t)\right)+\mathbf{A}^{T} \hat{\boldsymbol{\nu}} \\
& =t \nabla f_{0}\left(\mathbf{x}^{*}(t)\right)+\sum_{i=1}^{m} \frac{1}{-f_{i}\left(\mathbf{x}^{*}(t)\right)} \nabla f_{i}\left(\mathbf{x}^{*}(t)\right)+\mathbf{A}^{T} \hat{\boldsymbol{\nu}} .
\end{aligned}
$$

Define-se o par dual factível

$$
\begin{aligned}
\boldsymbol{\lambda}_{i}^{*}(t) & =-\frac{1}{t f_{i}\left(\mathbf{x}^{*}(t)\right)}, \quad i=1, \ldots, m, \\
\boldsymbol{\nu}^{*}(t) & =\hat{\boldsymbol{\nu}} / t
\end{aligned}
$$

Fica claro que $\boldsymbol{\lambda}_{i}^{*}(t) \succ 0$, pois $f_{i}\left(\mathbf{x}^{*}(t)\right)<0, i=1, \ldots, m$, assim safisfazendo a condição KKT (2.35). Substituindo (2.44) em (2.43), tem-se

$$
\nabla f_{0}\left(\mathbf{x}^{*}\right)+\sum_{i=1}^{m} \boldsymbol{\lambda}_{i}^{*} \nabla f_{i}\left(\mathbf{x}^{*}\right)+A^{T} \boldsymbol{\nu}^{*}=0
$$

satisfazendo a condição KKT (2.36).

Com isto, percebe-se que $\mathbf{x}^{*}(t)$ minimiza o Lagrangeano

$$
L(\mathbf{x}, \boldsymbol{\lambda}, \boldsymbol{\nu})=f_{0}(\mathbf{x})+\sum_{i=1}^{m} \boldsymbol{\lambda}_{i} f_{i}(\mathbf{x})+\boldsymbol{\nu}^{T}(\mathbf{A x}-\mathbf{b}),
$$

com $\boldsymbol{\lambda}_{i}=\boldsymbol{\lambda}_{i}^{*}(t), i=1, \ldots, m$, e $\boldsymbol{\nu}=\boldsymbol{\nu}^{*}(t)$ sendo o par dual factível. Portanto, a função dual $g\left(\boldsymbol{\lambda}_{i}^{*}(t), \boldsymbol{\nu}^{*}(t)\right)$ é finita e dada por

$$
\begin{aligned}
g\left(\boldsymbol{\lambda}_{i}^{*}(t), \boldsymbol{\nu}^{*}(t)\right) & =f_{0}\left(\mathbf{x}^{*}(t)\right)+\sum_{i=1}^{m} \boldsymbol{\lambda}_{i}^{*}(t) f_{i}\left(\mathbf{x}^{*}(t)\right)+\boldsymbol{\nu}^{*}(t)^{T}\left(\mathbf{A} \mathbf{x}^{*}(t)-\mathbf{b}\right) \\
g\left(\boldsymbol{\lambda}_{i}^{*}(t), \boldsymbol{\nu}^{*}(t)\right) & =f_{0}\left(\mathbf{x}^{*}(t)\right)+\sum_{i=1}^{m} \frac{1}{-t f_{i}\left(\mathbf{x}^{*}(t)\right)} f_{i}\left(\mathbf{x}^{*}(t)\right) \\
& =f_{0}\left(\mathbf{x}^{*}(t)\right)-m / t
\end{aligned}
$$

O gap dual associado com $\mathbf{x}^{*}(t)$ e o par dual $\boldsymbol{\lambda}^{*}(t)$ e $\boldsymbol{\nu}^{*}(t)$ é igual a $m / t$. Como consequência,

$$
f_{0}\left(\mathbf{x}^{*}(t)\right)-\mathcal{J}^{*} \leq m / t
$$

ou seja, $\mathbf{x}^{*}(t)$ é um $(m / t)$-subótimo do problema (2.33), que é equivalente ao problema

$$
\begin{array}{ll}
\text { minimize : } & (m / \epsilon) f_{0}(\mathbf{x})+\phi(\mathbf{x}) \\
\text { sujeito a : } & \mathbf{A x}=\mathbf{b},
\end{array}
$$


onde $\epsilon$ é a tolerância aceitável para o gap dual.

O Algoritmo 1, proposto inicialmente por Fiacco e McCormick (1990) com o nome de técnica sequencial de minimização irrestrita, calcula $\mathbf{x}^{*}(t)$ para uma sequência de valores crescentes de $t$, até $t \geq m / \epsilon$, garantindo que se tenha um $\epsilon$-subótima solução do problema original. Atualmente o método é usualmente chamado de método de barreira ou método de seguimento de caminho (WRIGHT, 1997).

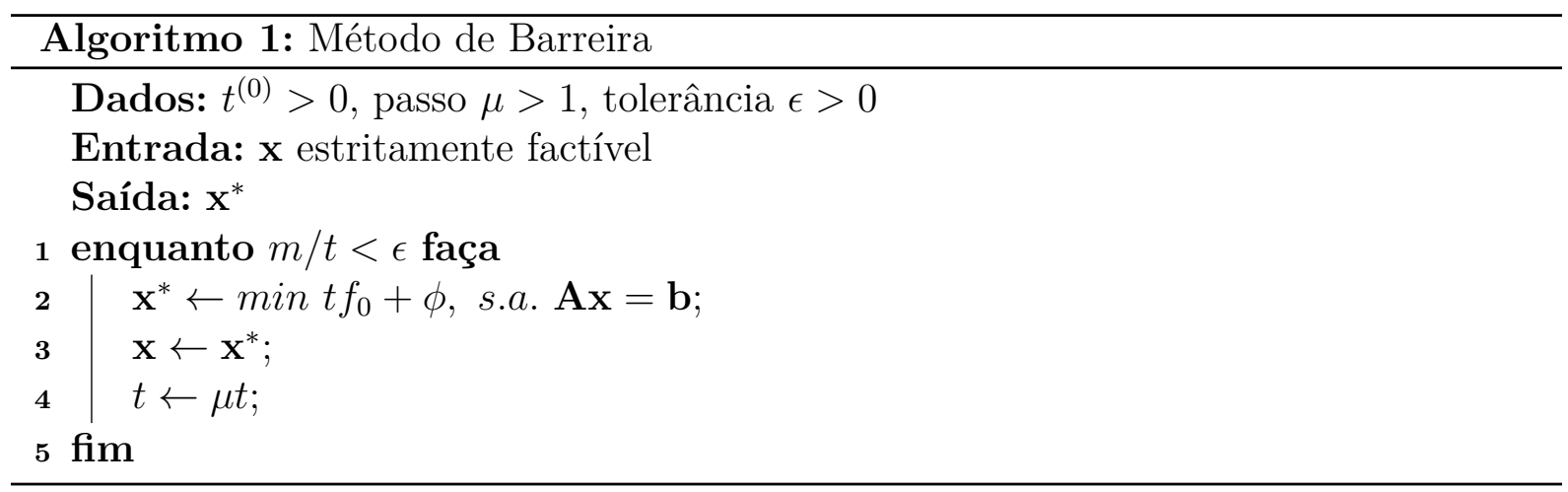

\subsubsection{Método do Ponto Interior para Problemas com Desigualdades Generalizadas}

Problemas com desigualdades generalizadas são da forma

$$
\begin{aligned}
\operatorname{minimize}: & f_{0}(\mathbf{x})=\mathcal{J}(\mathbf{x}) \\
\text { sujeito a }: & f_{i}(\mathbf{x}) \preceq_{K} 0, \quad i=1, \ldots, m \\
& \mathbf{A x}=\mathbf{b},
\end{aligned}
$$

onde $f_{0}: \mathbb{R}^{n} \rightarrow \mathbb{R}$ é convexa, $f_{i}: \mathbb{R}^{n} \rightarrow \mathbb{R}^{k_{i}}$ são funções $K_{i}$-convexas e $K_{i} \subseteq \mathbb{R}^{K_{i}}$ são cones próprios. Considera-se $f_{i}$ duplamente diferenciáveis, $\mathbf{A} \in \mathbb{R}^{p \times n} \operatorname{com} \operatorname{Posto}(\mathbf{A})=p<n$, e que o problema é solúvel.

Uma função $\psi: \mathbb{R}^{q} \rightarrow \mathbb{R}$ é dita um logarítmo generalizado para o cone próprio $K$ se

- $\psi$ é côncavo, fechado, duplamente diferenciável, dom $\psi=$ int $K$, e $\nabla^{2} \psi(\mathbf{y}) \prec 0$ para $\mathbf{y} \in \operatorname{int} K$;

- Existe $\theta>0$ tal que para todo $\mathbf{y} \succ_{K} 0$ e todo $s>0, \psi(s \mathbf{y})=\psi(\mathbf{y})+\theta \log s$.

em outras palavras, $\psi$ se comporta como um logarítmo através de qualquer raio do cone próprio $K$.

Define-se então a barreira logarítmica do problema (2.50) como sendo

$$
\phi(\mathbf{x})=-\sum_{i=1}^{m} \psi_{i}\left(-f_{i}(\mathbf{x})\right)
$$

com dom $\phi=\left\{\mathbf{x} \in \mathbb{R}^{n} \mid f_{i}(\mathbf{x}) \prec 0, i=1, \ldots, m\right\}$. O desenvolvimento do método para problemas com desigualdades generalizadas é paralelo ao caso das restrições escalares. 


\subsubsection{Método do Ponto Interior Primal-Dual}

O Método do Ponto Interior Primal-Dual (WRIGHT, 1997) é similar ao método de barreira, com algumas diferenças:

- Em cada iteração, ambas as variáveis primal e dual são atualizadas. Não existe distinção entre interações interiores e exteriores, como no método de barreira;

- A direção de procura é obtida por equações KKT modificadas considerando a função de barreira logarítmica generalizada, aplicando o Método de Newton a cada iteração;

- Pode-se iniciar em um ponto x não-factível.

As condições KKT modificadas podem ser expressas como $r_{t}(\mathbf{x}, \boldsymbol{\lambda}, \boldsymbol{\nu})=0$ tal que

$$
r_{t}(\mathbf{x}, \boldsymbol{\lambda}, \boldsymbol{\nu})=\left[\begin{array}{c}
\nabla f_{0}(\mathbf{x})+D f(\mathbf{x})^{T} \boldsymbol{\lambda}+\mathbf{A}^{T} \boldsymbol{\nu} \\
-\operatorname{diag}(\boldsymbol{\lambda}) f(\mathbf{x})-(a / t) \mathbf{I} \\
\mathbf{A} \mathbf{x}-\mathbf{b}
\end{array}\right]=\left[\begin{array}{c}
r_{\text {dual }} \\
r_{\text {cent }} \\
r_{\text {pri }}
\end{array}\right]
$$

onde o operador $D$ denota a matriz derivativa e os blocos $r_{d u a l}, r_{c e n t}$ e $r_{\text {prim }}$ se referem aos residuais primal, da condição de complementaridade e primal respectivamente. O passo do método de Newton é caracterizado pela equação linear

$$
r_{t}(\mathbf{y}+\Delta \mathbf{y}) \approx r_{t}(\mathbf{y})+D r_{t}(\mathbf{y}) \Delta \mathbf{y}=0
$$

i.e., $\delta \mathbf{y}=-D r_{t}(\mathbf{y})^{-1} r_{t}(\mathbf{y})$. Em termos de $\mathbf{x}, \boldsymbol{\lambda}$ e $\boldsymbol{\nu}$, temos

$$
\left[\begin{array}{ccc}
\nabla^{2}+\sum_{i=1}^{m} \boldsymbol{\lambda}_{i} \nabla^{2} f_{i}(\mathbf{x}) & D f(\mathbf{x})^{T} & \mathbf{A}^{T} \\
-\operatorname{diag}(\boldsymbol{\lambda}) D f(\mathbf{x}) & -\operatorname{diag}(f(\mathbf{x})) & 0 \\
\mathbf{A} & 0 & 0
\end{array}\right]\left[\begin{array}{c}
\Delta \mathbf{x} \\
\Delta \boldsymbol{\lambda} \\
\Delta \boldsymbol{\nu}
\end{array}\right]=\left[\begin{array}{c}
r_{d u a l} \\
r_{c e n t} \\
r_{\text {pri }}
\end{array}\right] .
$$

A direção de busca primal-dual $\Delta \mathbf{y}_{p d}=\left(\Delta \mathbf{x}_{p r}, \Delta \boldsymbol{\lambda}_{p r}, \Delta \boldsymbol{\nu}_{p r}\right)$ é definida como solução de (2.54). Define-se também o gap dual substituto, para cada $\mathbf{x}$ que satisfaça $f(\mathbf{x}) \prec 0$ e $\boldsymbol{\lambda} \succeq 0$ como $\hat{\boldsymbol{\eta}}(\mathbf{x}, \boldsymbol{\lambda})=-f(\mathbf{x})^{T} \boldsymbol{\lambda}$. Então, o algoritmo básico para o método de ponto interior primal-dual é apresentado no Algoritmo 2.

\subsubsection{Solvers para Otimização Convexa}

Em muitos casos, problemas genéricos de otimização podem ser reformulados em uma forma padrão de programação convexa (LP, QP, QCQP, SOCP ou SDP). Este processo de reformulação muitas vezes exige mudança de variáveis, uso de variáveis folgadas (slack variables), transformações geométricas, dentre outras técnicas apresentadas de forma ampla no livro de Boyd e Vandenberghe (2004). 


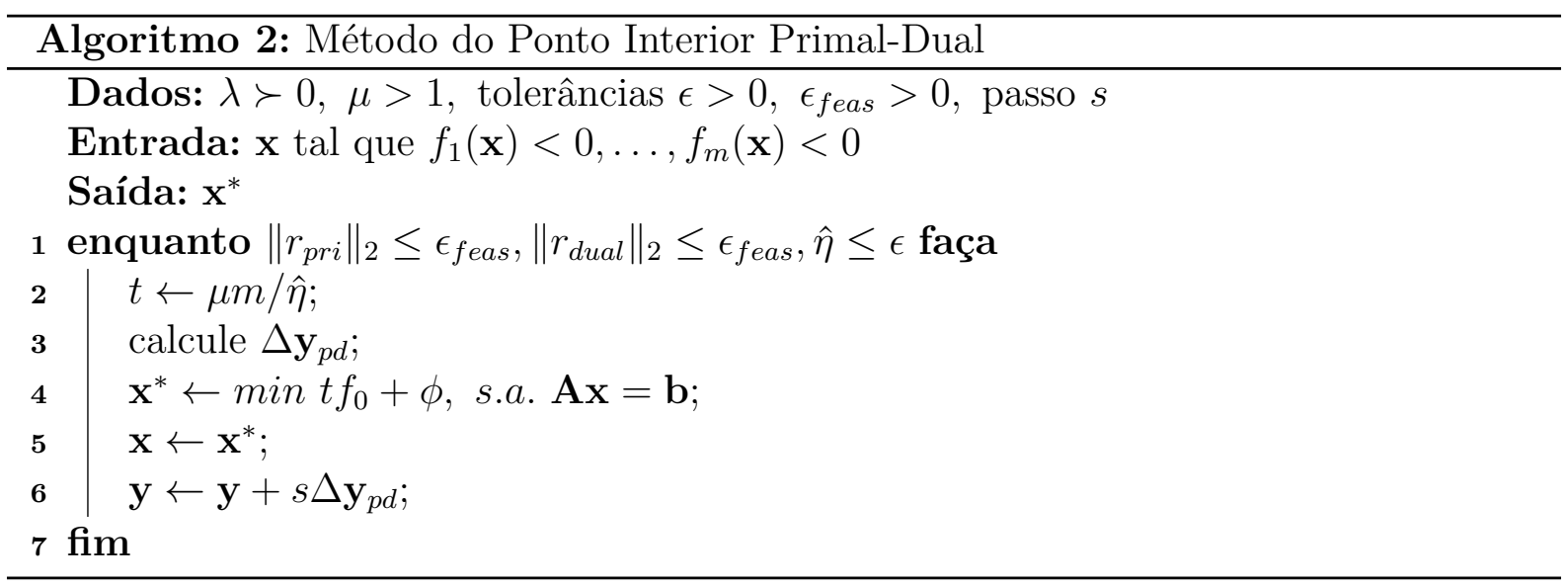

Uma vez reformulados como uma forma padrão, o problema de otimização convexa pode ser resolvido por um solver. Existem solvers disponíveis como programas específicos, mas tipicamente são subrotinas geradas para serem usadas em programas escritos em Fortran, C, Matlab ou Python.

Solvers baseados no Método do Ponto Interior Primal-Dual para programação semidefinida e programação cônica de segunda ordem estão disponíveis em pacotes como

- $C P L E X($ IBM, 2017);

- $C S D P$ (BORCHERS, 1999);

- DSDP5 (BENSON; YE; ZHANG, 2000);

- GuRoBi (GUROBI, 2017);

- SDPT3 (TOH; TODD; TüTüNCü, 1999);

- SeDuMi (STURM, 1999) e

- Mosek (ANDERSEN; ROOS; TERLAKY, 2003).

Para o uso dos solvers existem pacotes comerciais de modelagem algébrica com interfaces próprias, como o $A I M M S$, o $A M P L$ e o $G A M S$; ou como pacotes para o Matlab. Os mais utilizados são o YALMIP (LOFBERG, 2004) e o CVX (GRANT; BOYD, 2014). Este último é usado no Capítulo 3 para modelar o problema de otimização na etapa de Planejamento de Trajetória. 



\section{PLANEJAMENTO DE TRAJETÓRIA}

Como mostrado no Capítulo anterior, o planejamento ótimo de trajetória proposto por (VERSCHEURE et al., 2008) e posteriormente ampliado por (LIPP; BOYD, 2014) não inclui robôs subatuados. Neste Capítulo, uma adaptação desta proposta é apresentada para ser utilizada no planejamento ótimo de trajetória para um robô uniciclo diferencial, considerando como objetivos o tempo e a energia.

\subsection{DEFINIÇÃO DO PROBLEMA}

\subsubsection{Modelo dinâmico do robô diferencial}

Em um robô uniciclo diferencial, cada uma das duas rodas exerce uma força sobre todo o veículo, na direção de seus rolamentos, cujos módulos são dados por $F_{r}$ e $F_{l}$ relativos às rodas direita e esquerda, respectivamente, como ilustra a Figura 14.

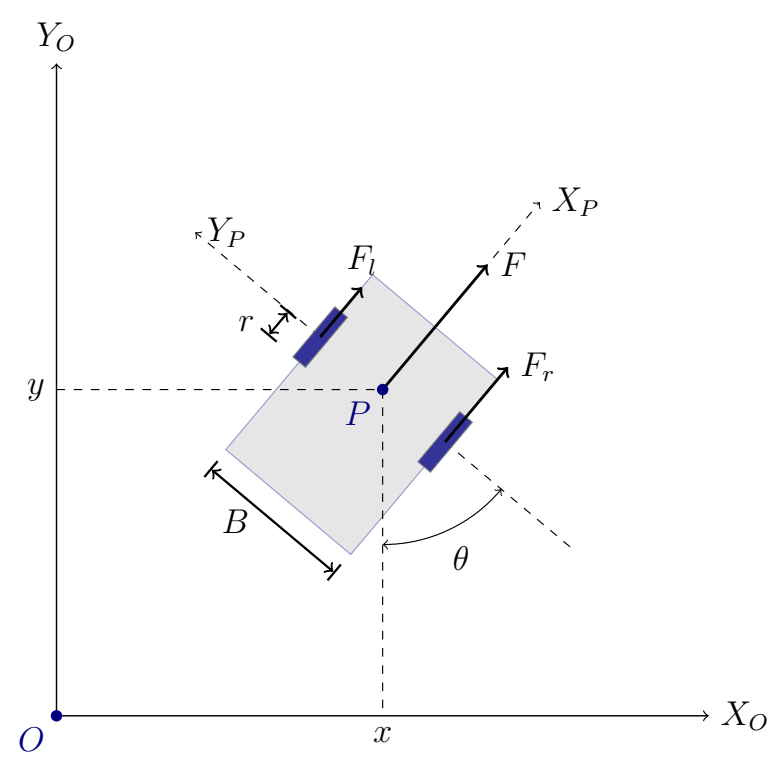

Figura 14 - O robô uniciclo diferencial, seus sistemas referenciais e sua composição de forças.

Fonte: o autor.

Como os rolamentos das rodas são paralelos entre si na direção $X_{P}$, a força resultante $F$ se dará também nesta direção, com seu módulo sendo

$$
F=\left(F_{r}+F_{l}\right)=\frac{K_{m}}{r}\left(u_{r}+u_{l}\right),
$$

onde $K_{m}$ é a constante de torque de ambos os motores (considerando que eles possuam as mesmas características eletromecânicas), enquanto $u_{r}$ e $u_{l}$ são as tensões aplicadas nos motores direito e esquerdo, respectivamente. 
Da mesma forma, o torque $T$ relativo às forças dos motores ao centro de rotação $P$ é produzido pelo binário dos torques $T_{r}$ e $T_{l}$ gerados pelas rodas direita e esquerda, respectivamente, da forma

$$
T=\left(T_{r}-T_{l}\right)=\frac{K_{m} B}{2 r}\left(u_{r}-u_{l}\right)
$$

No sistema de coordenadas global $\mathcal{F}_{O}$, dados a massa $m$ e o momento de inércia $J$ do conjunto, as equações referentes às Leis de Newton translacional e rotacional em relação às dimensões da configuração do veículo são:

$$
\begin{aligned}
F \cos (\theta) & =m \ddot{x}, \\
F \operatorname{sen}(\theta) & =m \ddot{y}, \\
T & =J \ddot{\theta} .
\end{aligned}
$$

A dinâmica da posição do ponto $P$ em $\mathcal{F}_{O}$ pode ser representada da forma matricial simplificada

$$
\mathbf{R}_{\mathbf{p}}(\theta) \mathbf{u}=m \ddot{\mathbf{p}}
$$

onde a matriz $\mathbf{R}_{\mathbf{p}}(\theta) \in \mathbb{R}^{2 \times 2}$ dada por

$$
\mathbf{R}_{\mathbf{p}}(\theta)=\left[\begin{array}{cc}
\frac{K_{m}}{r} \cos (\theta) & \frac{K_{m}}{r} \cos (\theta) \\
\frac{K_{m}}{r} \operatorname{sen}(\theta) & \frac{K_{m}}{r} \operatorname{sen}(\theta)
\end{array}\right]
$$

representa a rotação do vetor de entrada $\mathbf{u} \in \mathbb{R}^{2}, \mathbf{u}=\left[\begin{array}{ll}u_{r} & u_{l}\end{array}\right]^{T}$, relativo às tensões aplicadas nos motores das rodas direita e esquerda, respectivamente, enquanto o vetor $\ddot{\mathbf{p}} \in \mathbb{R}^{2}, \ddot{\mathbf{p}}=\left[\begin{array}{ll}\ddot{x} & \ddot{y}\end{array}\right]^{T}$ rege a dinâmica temporal da posição do ponto $P$ no plano.

De forma generalizada, a dinâmica da postura do robô no espaço de configuração pode ser escrita pela equação diferencial matricial

$$
\mathbf{R}_{\xi}(\theta) \mathbf{u}=\mathbf{M}_{\xi} \ddot{\xi}
$$

onde as matrizes $\mathbf{R}_{\xi}(\theta) \in \mathbb{R}^{3 \times 2}$ e $\mathbf{M}_{\xi} \in \mathbb{R}^{3 \times 3}$,

$$
\begin{aligned}
& \mathbf{R}_{\xi}(\theta)=\left[\begin{array}{c}
\mathbf{R}_{\mathbf{p}}(\theta) \\
\frac{K_{m} B}{2 r} \\
-\frac{K_{m} B}{2 r}
\end{array}\right], \\
& \mathbf{M}_{\xi}=\left[\begin{array}{cc|c}
m & 0 & 0 \\
0 & m & 0 \\
\hline 0 & 0 & J
\end{array}\right]
\end{aligned}
$$

representam a rotação do vetor de entrada e a inércia do robô, respectivamente, enquanto o vetor $\ddot{\boldsymbol{\xi}} \in \mathbb{R}^{3}, \ddot{\boldsymbol{\xi}}=\left[\begin{array}{lll}\ddot{x} & \ddot{y} & \ddot{\theta}\end{array}\right]^{T}$ rege a dinâmica de segunda ordem da postura do robô no domínio do tempo. 


\subsubsection{Cinemática do robô em um caminho}

Considere-se caminho a curva $\Gamma$ de classe $C^{n}$ em $\mathcal{F}_{O}, n \geq 2$, pela qual o robô deve se movimentar num intervalo de tempo $t=\left[0, T_{f}\right]$. O caminho $\Gamma$ pode ser definido por uma função vetorial $\mathbf{p}(t):\left[0, T_{f}\right] \rightarrow \mathbb{R}^{2}, \mathbf{p}(t)=[x(t) \quad y(t)]^{T}$, onde $x(t)$ e $y(t)$ são funções de classe $C^{2}$ que representam as coordenadas cartesianas do ponto $P$ no plano $\mathbb{R}^{2}$.

Lema 3.1 (Coordenadas cartesianas e generalizadas). Se a velocidade linear do robô for não-negativa, um caminho $\Gamma$ descrito pelas posições do robô $\mathbf{p}(t)=\left[\begin{array}{ll}x(t) & y(t)\end{array}\right]^{T}$ no espaço cartesiano define também suas posturas $\boldsymbol{\xi}(t)=\left[\begin{array}{lll}x(t) & y(t) & \theta(t)\end{array}\right]^{T}$ no espaço generalizado de configuração.

Demonstração. Como a função $\mathbf{p}(t)$ que descreve o caminho $\Gamma$ é uma curva de classe $C^{2}$, a sua derivada em relação ao tempo $\dot{\mathbf{p}}(t)=\left[\begin{array}{ll}\dot{x}(t) & \dot{y}(t)\end{array}\right]^{T}$ existe e é contínua.

Da restrição não-holonômica apresentada pela Equação 2.15, se $v(t)>0$, tem-se que o ângulo $\theta(t)$ é dado por

$$
\theta(t)=t g^{-1}\left(\frac{\dot{y}(t)}{\dot{x}(t)}\right),
$$

e é função contínua $\theta:\left[0, T_{f}\right] \rightarrow[0,2 \pi]$.

Portanto, o caminho também pode ser representado pela função parametrizada no tempo das posturas do robô $\boldsymbol{\xi}(t):\left[\begin{array}{l}0, T_{f}\end{array}\right] \rightarrow \mathbb{R}^{3}, \boldsymbol{\xi}(t)=\left[\begin{array}{lll}\mathbf{p}(\mathbf{t}) & \theta(t)\end{array}\right]^{T}=\left[\begin{array}{lll}x(t) & y(t) & \theta(t)\end{array}\right]^{T}$, desde que $v(t)>0$ para todo $t \in\left[0, T_{f}\right]$, que define as configurações generalizadas do robô parametrizadas no tempo $t$.

Agora seja a função avanço, $\gamma(t): \mathbb{R} \rightarrow \mathbb{R}$, definida por

$$
\gamma(t)=\int_{0}^{t} v(\iota) d \iota=\int_{0}^{t} \sqrt{\dot{x}^{2}(\iota)+\dot{y}^{2}(\iota)} d \iota
$$

que representa geometricamente a distância percorrida pelo robô no caminho $\Gamma$ dado por $\mathbf{p}(t)$ no intervalo de tempo $\iota \in[0, t]$.

Lema 3.2 (Coordenadas alternativas). Se a velocidade linear do robô for não-negativa, um caminho $\Gamma$ descrito pelas posições do robô $\mathbf{p}(t)=\left[\begin{array}{ll}x(t) & y(t)\end{array}\right]^{T}$ no espaço cartesiano é descrito de forma equivalente pela função parametrizada no tempo $\mathbf{q}(t):\left[0, T_{f}\right] \rightarrow \mathbb{R}^{2}$,

$$
\mathbf{q}(t)=\left[\begin{array}{ll}
\gamma(t) & \theta(t)
\end{array}\right]^{T}
$$

se conhecida a posição inicial do robô $\mathbf{p}_{0}=\left[\begin{array}{ll}x_{0} & y_{0}\end{array}\right]^{T}$.

Demonstração. Como a função $\mathbf{p}(t)$ que descreve o caminho $\Gamma$ é uma curva de classe $C^{2}$, a sua derivada em relação ao tempo $\dot{\mathbf{p}}(t)=\left[\begin{array}{ll}\dot{x}(t) & \dot{y}(t)\end{array}\right]^{T}$ existe e é contínua. 
Então a função avanço $\gamma(t)=\gamma(\mathbf{p}(t))$ descrita pela Equação 3.10 existe e é contínua no intervalo de tempo $\left[0, T_{f}\right]$. Pelo Lema 3.1, a função $\theta(t)=\theta(\mathbf{p}(t))$ descrita pela Equação 3.9 também existe e é contínua no mesmo intervalo de tempo $\left[0, T_{f}\right]$.

Além disso, se conhecida a posição inicial $\mathbf{p}_{0}=\left[\begin{array}{ll}x_{0} & y_{0}\end{array}\right]^{T}$, as coordenadas da posição $\mathbf{p}(t)$ do robô no tempo $t$ podem ser calculadas por

$$
\begin{aligned}
& x(t)=x_{0}+\int_{0}^{t} \gamma(\iota) \cos \theta(\iota) d \iota, \\
& y(t)=y_{0}+\int_{0}^{t} \gamma(\iota) \operatorname{sen} \theta(\iota) d \iota .
\end{aligned}
$$

Então, como $\mathbf{q}(t)=\mathbf{q}(\mathbf{p}(t))$ e $\mathbf{p}(t)=\mathbf{p}(\mathbf{q}(t))$, as descrições da curva $\Gamma$ por $\mathbf{p}(t)$ e por $\mathbf{q}(t)$ são equivalentes.

\subsubsection{Dinâmica do robô em um caminho}

O ângulo de orientação $\theta$ define a direção do deslocamento do robô e, portanto, de sua velocidade linear $v(t)=\dot{\gamma}(t)$. A dinâmica temporal do vetor q é dada pelas Leis de Newton representadas pelas equações diferenciais

$$
\begin{aligned}
F=\frac{K_{m}}{r}\left(u_{r}+u_{l}\right) & =m \ddot{\gamma}, \\
T=\frac{K_{m} B}{2 r}\left(u_{r}-u_{l}\right) & =J \ddot{\theta},
\end{aligned}
$$

que pode ser escrita da forma matricial

$$
\mathbf{R}_{\mathrm{q}} \mathbf{u}=\mathbf{M}_{\mathrm{q}} \ddot{\mathbf{q}}
$$

tal que

$$
\begin{aligned}
& \mathbf{R}_{\mathbf{q}}=\left[\begin{array}{cc}
\frac{K_{m}}{r} & \frac{K_{m}}{r} \\
\frac{K_{m} B}{2 r} & -\frac{K_{m} B}{2 r}
\end{array}\right], \\
& \mathbf{M}_{\mathbf{q}}=\left[\begin{array}{ll}
m & 0 \\
0 & J
\end{array}\right],
\end{aligned}
$$

onde o vetor $\ddot{\mathbf{q}} \in \mathcal{R}^{2}, \ddot{\mathbf{q}}=\left[\begin{array}{ll}\ddot{\gamma} & \ddot{\theta}\end{array}\right]^{T}$ rege a dinâmica de segunda ordem da configuração generalizada alternativa do robô no domínio do tempo.

Note que, ao contrário das matrizes de rotação de entrada para as equações dinâmicas da posição (3.5) e da postura (3.7) do robô, a matriz de rotação de entrada da equação dinâmica da configuração alternativa (3.16) é independente do ângulo $\theta$.

Note também que a dimensionalidade da dinâmica do robô, quando representada pela configuração generalizada alternativa (3.15), permite a sua controlabilidade, pois a restrição não-holonômica fica implícita no caminho percorrido. 


\subsubsection{Parametrização geométrica do caminho}

A função paramétrica $\tau:\left[0, T_{F}\right] \rightarrow[0,1]$, neste trabalho denominado tempo virtual, mapeia o tempo de movimento do robô em um intervalo normalizado, onde $\tau(0)=0$ e $\tau\left(T_{F}\right)=1$. Esta função é monótona crescente, isto é $\dot{\tau}(t)>0$, em todo intervalo $\tau=[0,1]$.

Em coordenadas cartesianas em $\mathcal{F}_{O}$, o caminho pode ser definido por uma função $\mathbf{p}:[0,1] \rightarrow \mathbb{R}^{2}, \mathbf{p}(\tau)=[x(\tau) \quad y(\tau)]^{T}$. Pelas transformações (3.9) e (3.10), define-se o caminho parametrizado geometricamente pela função $\mathbf{s}:[0,1] \rightarrow \mathbb{R}^{2}$,

$$
\mathbf{s}(\tau)=\left[\begin{array}{ll}
\gamma(\tau) & \theta(\tau)
\end{array}\right]^{T}
$$

Deseja-se que a configuração alternativa do robô no tempo siga o caminho definido, como ilustrado na Figura 15. ou seja,

$$
\mathbf{q}(t)=\mathbf{s}(\tau(t)), \quad t \in\left[0, T_{F}\right] .
$$

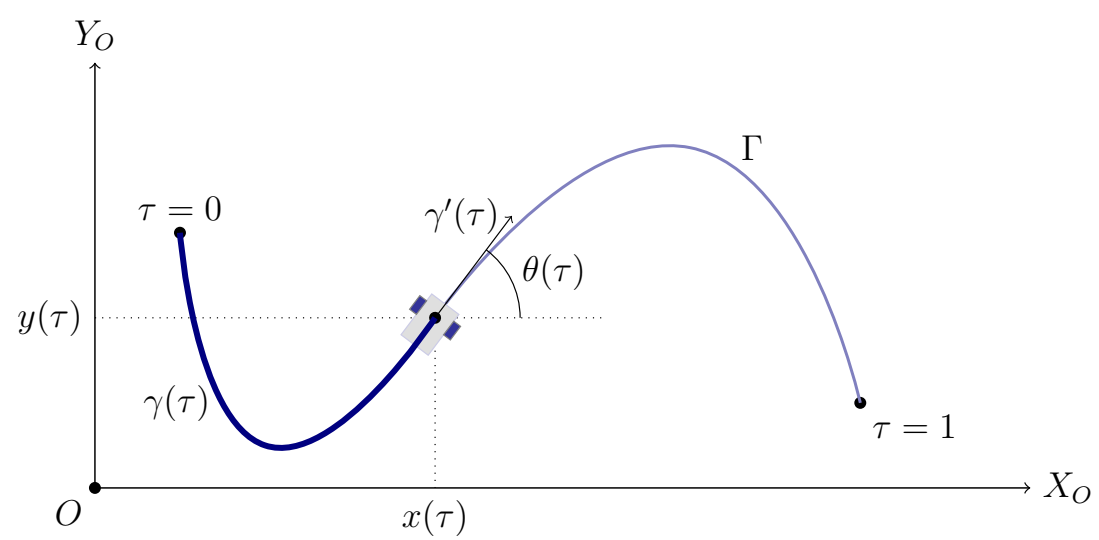

Figura 15 - A posição $p$ do robô uniciclo diferencial e seu caminho de referência $s$ parametrizada em $\tau(t)$. A linha contínua ilustra a curva $\Gamma$ em $\mathcal{F}_{O}$ no intervalo de tempo virtual $[0,1]$, enquanto a linha mais espessa mostra o avanço $\gamma$ no intervalo de tempo virtual $[0, \tau]$.

Fonte: o autor.

As derivadas da equação (3.19) fornecem a velocidade e a aceleração do robô no plano pela regra da cadeia:

$$
\begin{aligned}
\dot{\mathbf{q}}(t) & =\mathbf{s}^{\prime}(\tau) \dot{\tau}(t) \\
\ddot{\mathbf{q}}(t) & =\mathbf{s}^{\prime}(\tau) \ddot{\tau}(t)+\mathbf{s}^{\prime \prime}(\tau) \dot{\tau}^{2}(t),
\end{aligned}
$$

onde a notação $(.)^{\prime}$ refere-se à derivada em relação à variável $\tau$.

\subsubsection{Problema de otimização Tempo-Energia}

Seja um robô diferencial definido em 3.1.1, cuja configuração inicial é dada por $\mathbf{q}(0)=\mathbf{q}_{0}$, movimentando-se num caminho definido por $\mathbf{s}(\tau(t))$ durante um intervalo de tempo $\left\{t \in \mathbb{R} \mid t=\left[0, T_{F}\right]\right\}$, até alcançar uma configuração final $\mathbf{q}\left(T_{F}\right)=q_{F}$. 
Durante este movimento, o robô está limitado a velocidades e acelerações máximas, e também às tensões em seus motores, respeitando certos limites.

Deseja-se encontrar um perfil de velocidade $\dot{\mathbf{q}}(t), t \in\left[0, T_{F}\right]$, tal que o robô percorra o caminho $\mathbf{s}$ de forma eficiente, ou seja, em um menor tempo $T_{F}$ e com menor consumo energético possível. Tal problema pode ser definido como um problema de otimização descrito da seguinte forma:

Problema 3.1. (Problema de mínimo Tempo-Energia)

$$
\begin{aligned}
\underset{\left(\mathbf{u}, T_{f}\right)}{\operatorname{minimize}:} \quad & \mathcal{J}(t, \mathbf{u})=\int_{0}^{T_{F}}\|\mathbf{u}(t)\|_{2}^{2} d t+\mu \int_{0}^{T_{F}} d t \\
\text { sujeito } a: \quad & \mathbf{R}_{\mathbf{q}} \mathbf{u}(t)=\mathbf{M}_{\mathbf{q}} \ddot{\mathbf{q}}(t) \\
\mathbf{q}(t) & =\mathbf{s}(\tau(t)) \\
\mathbf{u}_{\min } & \leq \mathbf{u}(t) \leq \mathbf{u}_{\text {max }} \\
\dot{\mathbf{q}}_{\text {min }} & \leq \dot{\mathbf{q}}(t) \leq \dot{\mathbf{q}}_{\text {max }} \\
\ddot{\mathbf{q}}_{\text {min }} & \leq \ddot{\mathbf{q}}(t) \leq \ddot{\mathbf{q}}_{\text {max }}, \quad t \in\left[0, T_{F}\right]
\end{aligned}
$$

A Função Custo $\mathcal{J}(3.22)$ possui dois termos integrais. O primeiro termo é relativo à energia consumida durante o intervalo de tempo $\left[0, T_{f}\right]$, dada pela integração da potência desenvolvida pelos motores elétricos do robô. Minimizar a potência é equivalente a minimizar o quadrado das funções $u_{r}(t)$ e $u_{l}(t)$, considerando iguais as impedâncias dos motores elétricos. O segundo termo é o próprio tempo final $T_{f}$, ponderado pelo coeficiente $\mu \in \mathbb{R}_{+}$.

As variáveis de otimização do problema são o tempo final $T_{F}$ e o vetor de entrada $\mathbf{u}$, enquanto a matrizes $\mathbf{R}_{\mathbf{q}}$ e $\mathbf{M}_{\mathbf{q}}$, e o caminho $\mathbf{s}$ são dados do problema.

A equação (3.23) impôe como restrição do problema a dinâmica do robô, enquanto a equação (3.24) impõe que o robô deverá percorrer o caminho s. As notações $(.)_{\text {min }}$ e $(.)_{\max }$ denotam o limite mínimo e máximo da variável respectivamente, e as desigualdades (3.25-3.27) definem o poliedro $\mathcal{C}_{t} \subseteq \mathbb{R}^{2 x 2 x 2}$ tal que $(\mathbf{u}(t), \dot{\mathbf{q}}(t), \ddot{\mathbf{q}}(t)) \in \mathcal{C}_{t}$.

\subsection{CONVEXIFICAÇÃO}

Substituindo a equação diferencial (3.21) na dinâmica da postura alternativa definida pela equação (3.15), pode-se escrever (VERSCHEURE et al., 2008; LIPP; BOYD, 2014):

$$
\mathbf{R}_{\mathbf{q}}(\tau) \mathbf{u}(\tau)=\mathbf{M}_{\mathbf{q}}\left(\mathbf{s}^{\prime}(\tau) \ddot{\tau}(t)+\mathbf{s}^{\prime \prime}(\tau) \dot{\tau}^{2}(t)\right)
$$


onde os vetores $\mathbf{s}^{\prime}(\tau)$ e $\mathbf{s}^{\prime \prime}(\tau)$ podem ser avaliadas a cada tempo virtual $\tau$, pois o caminho $\mathbf{s}(\tau)$ é definido no problema.

Introduzem-se também duas novas funções escalares ao problema:

$$
\begin{aligned}
& a(\tau)=\ddot{\tau}, \\
& b(\tau)=\dot{\tau}^{2},
\end{aligned}
$$

que descrevem a aceleração e o quadrado da velocidade do veículo no domínio do tempo virtual sucessivamente. Observando que

$$
\begin{aligned}
& \dot{b}(\tau)=b^{\prime}(\tau) \dot{\tau}, \\
& \dot{b}(\tau)=\frac{d\left(\dot{\tau}^{2}\right)}{d t}=2 \ddot{\tau} \dot{\tau}=2 a(\tau) \dot{\tau},
\end{aligned}
$$

extrai-se a relação entre as funções

$$
b^{\prime}(\tau)=2 a(\tau) .
$$

A função objetivo (3.22) pode ser redefinida, usando a equação (3.30), como:

$$
\mathcal{J}(\tau, \mathbf{u})=\int_{\tau(0)}^{\tau\left(T_{F}\right)}\left[\|\mathbf{u}(\tau)\|_{2}^{2}+\mu\right] \frac{d \tau}{\dot{\tau}}=\int_{0}^{1} \frac{\left[\|\mathbf{u}(\tau)\|_{2}^{2}+\mu\right]}{\sqrt{b(\tau)}} d \tau,
$$

e o Problema 3.1 pode ser então reescrito como:

Problema 3.2. (Problema convexo de mínimo Tempo-Energia)

$$
\begin{aligned}
\underset{(\mathbf{u}, a, b)}{\operatorname{minimize}:} & \mathcal{J}(\tau, \mathbf{u})=\int_{0}^{1} \frac{\left[\|\mathbf{u}(\tau)\|_{2}^{2}+\mu\right]}{\sqrt{b(\tau)}} d \tau \\
\text { sujeito } a: \quad & \mathbf{R}_{\mathbf{q}}(\tau) \mathbf{u}(\tau)=\mathbf{M}_{\mathbf{q}}\left(\mathbf{s}^{\prime}(\tau) a(\tau)+\mathbf{s}^{\prime \prime}(\tau) b(\tau)\right) \\
b^{\prime}(\tau) & =2 a(\tau) \\
(\mathbf{u}(\tau), a(\tau), b(\tau)) & \in \mathcal{C}_{\tau}, \quad \tau \in[0,1],
\end{aligned}
$$

onde $\mathcal{C}_{\tau} \subseteq \mathbb{R}^{2 \times 3 x 3}$ é um conjunto limitado do domínio do problema, mapeado pelas transformações dadas por (3.20-3.21) e pela definição das variáveis dadas por (3.29-3.30), tal que $\mathcal{C}_{\tau}=\left\{(\mathbf{u}(\tau), a(\tau), b(\tau)) \mid\left(\mathbf{u}(\tau), \mathbf{s}^{\prime 2}(\tau) b(\tau), \mathbf{s}^{\prime}(\tau) a(\tau)+\mathbf{s}^{\prime \prime}(\tau) b(\tau)\right) \in \mathcal{C}_{t}\right\}$.

\subsubsection{Análise de convexidade}

Um problema de otimização convexa é um problema da forma:

$$
\begin{aligned}
\underset{x}{\operatorname{minimize}:} & \mathcal{J}(x) \\
\text { sujeito a : } & f_{i}(x) \leq 0, \quad i=1, \ldots, m \\
& a_{j}^{T} x=b_{j}, \quad j=1, \ldots, n,
\end{aligned}
$$


onde tanto a função objetivo $\mathcal{J}$ quanto as funções de restrição de desigualdade $f_{1}, \ldots f_{m}$ devem ser funções convexas, e as funções de restrição de igualdade $h_{j}(x)=a_{j}^{T} x-b_{j}$ devem ser funções afins.

Teorema 3.1 (Condição de Segunda Ordem). A função $f$ é convexa se e somente se domf é convexo e sua Hessiana é semidefinida positiva, ou seja, para todo $x \in \operatorname{domf}$, $\nabla^{2} f(x) \succeq 0$, cuja condição necessária e suficiente é que possua todos os autovalores não-negativos (BOYD; VANDENBERGHE, 2004).

\subsubsection{Convexidade da função objetivo}

Reescrevendo a função objetivo do Problema 3.2, dada pela equação (3.32):

$$
\mathcal{J}=\int_{0}^{1} F_{1} d \tau+\mu \int_{0}^{1} F_{2} d \tau
$$

onde $F_{1}=b^{-\frac{1}{2}}(\tau) \mathbf{u}(\tau)^{T} \mathbf{u}(\tau)$ e $F_{2}=b^{-\frac{1}{2}}(\tau)$. A função $F_{2}$ é uma função da forma $x^{a}, a \leq 0$ e, portanto, convexa em $\mathbb{R}_{++}$.

Lema 3.3. A função $F_{1}: \mathbb{R}_{+} \times \mathbb{R} \rightarrow \mathbb{R}, F_{1}=b^{-\frac{1}{2}}(\tau)\|\mathbf{u}(\tau)\|_{2}^{2}$ é convexa em seu domínio.

Demonstração. Como $\mathbf{u}(\tau)=\left[u_{r}(\tau) u_{l}(\tau)\right]^{T}$, considere-se a seguinte relação:

$$
\frac{d}{d \tau}\left(\|\mathbf{u}(\tau)\|_{2}^{2}\right)=\frac{d}{d \tau}\left(\mathbf{u}(\tau)^{T} \mathbf{u}(\tau)\right)=\frac{d}{d \tau}\left(u_{r}(\tau)^{2}+u_{l}(\tau)^{2}\right)=2\left(u_{r}(\tau)+u_{l}(\tau)\right)=2\|\mathbf{u}(\tau)\|_{1}
$$

A Hessiana da função $F_{1}$, escrita considerando a relação (3.37),

$$
\mathbf{H}=\nabla^{2} F_{1}(b, \mathbf{u})=\left[\begin{array}{cc}
\frac{3}{4} b^{-\frac{5}{2}}\|\mathbf{u}(\tau)\|_{2}^{2} & -b^{-\frac{3}{2}}\|\mathbf{u}(\tau)\|_{1} \\
-b^{-\frac{3}{2}}\|\mathbf{u}(\tau)\|_{1} & 2 b^{-\frac{1}{2}}(\tau)
\end{array}\right],
$$

é uma matriz simétrica hermitiana. Seja $\lambda_{1}$ e $\lambda_{2}$ os autovalores da matriz $\mathbf{H}$. Como $\operatorname{det}(\mathbf{H})=\lambda_{1} \lambda_{2}$, para que todos os autovalores de $\mathbf{H}$ sejam não-negativos, é necessário que $\operatorname{det}(\mathbf{H}) \geq 0$. Por outro lado, se o determinante é não-negativo, então (i) ambos os autovalores são positivos ou (ii) ambos os autovalores são negativos. Como $\operatorname{traço}(\mathbf{H})=$ $\lambda_{1}+\lambda_{2}$, se $\operatorname{det}(\mathbf{H}) \geq 0$ e $\operatorname{traço}(\mathbf{H}) \geq 0$, ambos os autovalores serão não-negativos.

Como $b \in \mathbb{R}_{+},\|\mathbf{u}\|_{1} \in \mathbb{R}$, tem-se:

$$
\begin{aligned}
\operatorname{det}(\mathbf{H}) & =\frac{3}{2} b^{-3}(\tau)\|\mathbf{u}(\tau)\|_{2}^{2}+b^{-3}(\tau)\|\mathbf{u}(\tau)\|_{2}^{2}=\frac{\|\mathbf{u}(\tau)\|_{2}^{2}}{2 b^{3}(\tau)} \geq 0 \\
\operatorname{traço}(\mathbf{H}) & =\frac{3}{4} b^{-\frac{5}{2}}\|\mathbf{u}(\tau)\|_{2}^{2}+2 b^{-\frac{1}{2}}(\tau) \geq 0,
\end{aligned}
$$

então, $\mathbf{H}=\nabla^{2} F_{1}(b, \mathbf{u}) \geq 0$. Portanto, pelo Teorema 3.1, a função $F_{1}$ é convexa em $\mathbb{R}_{+} \times \mathbb{R} \rightarrow \mathbb{R}$. 
Como operações de soma ponderada com reais não-negativos, assim como operação de integração preserva a convexidade, e como $F_{1}$ e $F_{2}$ são funções convexas em $\mathbb{R}_{+}$e $\mathbb{R}_{++}$ respectivamente, a função objetivo $\mathcal{J}=\mathcal{J}(\tau, u)$ da equação 3.36 é convexa em $\mathbb{R}_{+} \times \mathbb{R}_{+}$

\subsubsection{Afinidade das restrições de igualdade}

A dinâmica do veículo impõe a restrição de igualdade (3.33), que é uma função afim em $\mathbf{u}(\tau), a(\tau)$ e $b(\tau)$ e, portanto, convexa; e a relação entre $a(\tau)$ e $b(\tau)$ na restrição (3.34) é uma operação de diferenciação, portanto, é um operador linear que preserva a convexidade.

\subsubsection{Convexidade das restrições de desigualdade}

O conjunto de pontos $(\mathbf{u}, a, b)$ tais que a restrição (3.35) se mantém inativa, isto é, mantém o problema de otimização possível, pertence a um conjunto $\mathcal{C}_{t}$ tal que

$$
\mathcal{C}_{t}=\bigcap_{i=1}^{m} \operatorname{dom} f_{i},
$$

onde as $m=6$ funções de desigualdade $f_{i}$

$$
\begin{array}{ll}
f_{1}=\mathbf{u}(\tau)-\mathbf{u}_{\max }, & f_{2}=\mathbf{u}_{\min }-\mathbf{u}(\tau), \\
f_{3}=\mathbf{s}^{\prime 2}(\tau) b(\tau)-\dot{\mathbf{q}}_{\max }^{2}, & f_{4}=\dot{\mathbf{q}}_{\min }^{2}-\mathbf{s}^{\prime 2}(\tau) b(\tau), \\
f_{5}=\mathbf{s}^{\prime}(\tau) a(\tau)+\mathbf{s}^{\prime \prime}(\tau) b(\tau)-\ddot{\mathbf{q}}_{\max }, & f_{6}=\ddot{\mathbf{q}}_{\text {min }}-\mathbf{s}^{\prime}(\tau) a(\tau)+\mathbf{s}^{\prime \prime}(\tau) b(\tau) .
\end{array}
$$

são funções afins de $(\mathbf{u}, a, b)$ e, portanto, convexas.

\subsection{LINEARIZAÇÃO DISCRETA}

O Problema 3.2 tem como variável independente o tempo virtual $\tau(t)$. Para resolver numericamente o problema, se fazem necessário reformular as equações (3.32) - (3.35) como equações discretas. Para tanto, a variável $\tau$ é discretizada em $N+1$ pontos, de tal forma que $\tau_{0}=0 \leq \tau_{i} \leq 1=\tau_{N}$, para $i=1, \ldots, N-1$.

Da mesma forma, as variáveis $\mathbf{u}(\tau), a(\tau), b(\tau)$ deverão ser discretizadas introduzindo um número finito de variáveis, respectivamente $\mathbf{u}_{i} \in \mathbb{R}^{2} ; a_{i} \in \mathbb{R}, b_{i} \in \mathbb{R} . i=1, \ldots, N$; assim como a variável $\mathbf{s}(\tau)$ e suas derivadas de primeira e segunda ordem em relação ao tempo virtual $\tau$.

\subsubsection{Discretização das variáveis de otimização}

Considerando os intervalos de discretização pequenos o suficiente, pode-se também considerar que as variáveis $a(\tau)$ e $\mathbf{u}(\tau)$ são constantes no intervalo entre dois pontos discretos consecutivos $\tau_{i-1}$ e $\tau_{i}, i=1, \ldots, N$. Portanto, tanto $a(\tau)$ quanto $\mathbf{u}(\tau)$ são 
descontínuas no tempo virtual $\tau$. Estas variáveis são então avaliadas no ponto médio entre dois pontos consecutivos

$$
\bar{\tau}_{i}=\frac{\tau_{i}+\tau_{i-1}}{2}
$$

de tal forma que

$$
a_{i}=a\left(\bar{\tau}_{i}\right)=a\left(\frac{\tau_{i}+\tau_{i-1}}{2}\right)
$$

como ilustra a Figura 16; assim como

$$
\mathbf{u}_{i}=\mathbf{u}\left(\bar{\tau}_{i}\right)=\mathbf{u}\left(\frac{\tau_{i}+\tau_{i-1}}{2}\right)
$$

para todo $i=1, \ldots, N$.

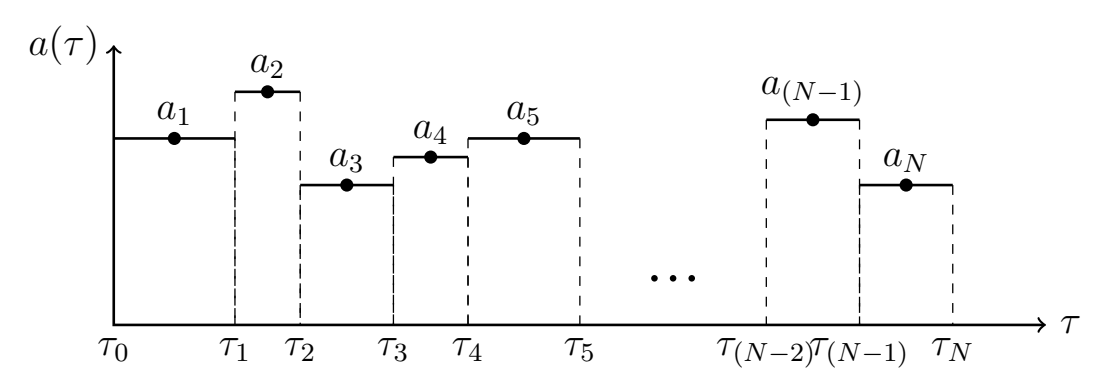

Figura $16-a(\tau)$ é constante por partes para $\tau \in[0,1]$.

Fonte: adaptado de Verscheure et al. (2009)

A variável $b$ é avaliada em cada ponto discreto da forma $b_{i}=b\left(\tau_{i}\right), i=1, \ldots, N$. O valor de $b_{0}=b\left(\tau_{0}\right)=\left(\frac{d \tau(0)}{d t}\right)^{2}$, e o quadrado da velocidade inicial do veículo deve ser definido como parâmetro do problema. Como assume-se que $a(\tau)$ é constante, tem-se que a variável $b(\tau)$ é afim entre dois intervalos consecutivos, e em expansão de Taylor de primeira ordem estima-se:

$$
b(\tau)=b_{i-1}+\left(\frac{b_{i}-b_{i-1}}{\tau_{i}-\tau_{i-1}}\right)\left(\tau-\tau_{i-1}\right),
$$

para todo $i=1, \ldots, N$, como ilustrado na Figura 17, e, no ponto médio entre dois tempos de discretização, tem-se

$$
b\left(\bar{\tau}_{i}\right)=\frac{b_{i}+b_{i-1}}{2}
$$

para todo $i=1, \ldots, N$. 


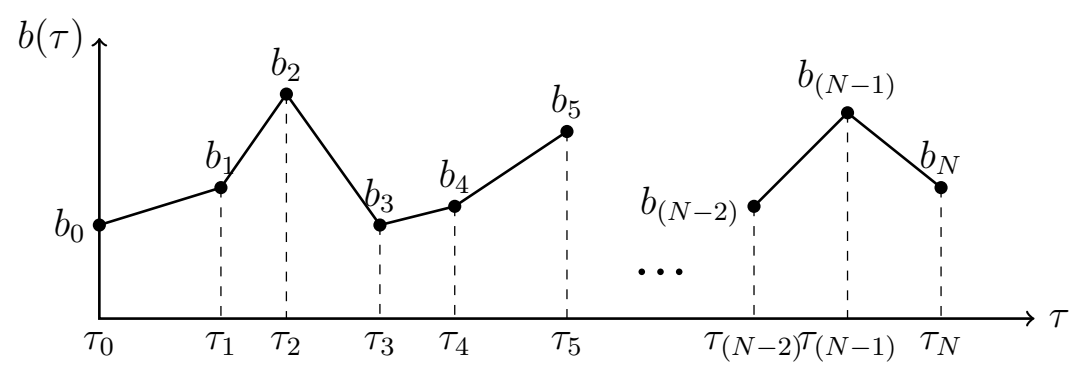

Figura $17-b(\tau)$ é linear por partes para $\tau \in[0,1]$. Fonte: adaptado de Verscheure et al. (2009)

\subsubsection{Discretização das equações do problema de otimização}

A função objetivo do Problema 3.2 descrita pela equação (3.32) pode ser reescrita como um somatório de integrais definidas em cada intervalo de discretização da seguinte forma:

$$
\mathcal{J}(\tau, \mathbf{u})=\int_{0}^{1} \frac{\left[\|\mathbf{u}(\tau)\|_{2}^{2}+\mu\right]}{\sqrt{b(\tau)}} d \tau=\sum_{i=1}^{N} \int_{\tau_{i-1}}^{\tau_{i}} \frac{\left[\|\mathbf{u}(\tau)\|_{2}^{2}+\mu\right]}{\sqrt{b(\tau)}} d \tau
$$

Como $u(\tau)$ é constante no intervalo de discretização,

$$
\mathcal{J}(\tau, u)=\sum_{i=1}^{N}\left\{\left(\left\|\mathbf{u}_{i}\right\|_{2}^{2}+\mu\right) \int_{\tau_{i-1}}^{\tau_{i}} \frac{1}{\sqrt{b(\tau)}} d \tau\right\} .
$$

Substituindo a equação (3.43), tem-se

$$
\begin{aligned}
\mathcal{J}(\tau, u) & =\sum_{i=1}^{N}\left(\left\|\mathbf{u}_{i}\right\|_{2}^{2}+\mu\right)\left\{\int_{\tau_{i-1}}^{\tau_{i}}\left[b_{i-1}+\left(\frac{b_{i}-b_{i-1}}{\tau_{i}-\tau_{i-1}}\right)\left(\tau-\tau_{i-1}\right)\right]^{-1 / 2} d \tau\right\}= \\
& =\sum_{i=1}^{N}\left(\left\|\mathbf{u}_{i}\right\|_{2}^{2}+\mu\right)\left\{\left.2 \frac{\left[b_{i-1}+\left(\frac{b_{i}-b_{i-1}}{\tau_{i}-\tau_{i-1}}\right)\left(\tau-\tau_{i-1}\right)\right]^{1 / 2}}{\left(\frac{b_{i}-b_{i-1}}{\tau_{i}-\tau_{i-1}}\right)}\right|_{\tau_{i-1}} ^{\tau_{i}}\right\}= \\
& =\sum_{i=1}^{N}\left(\left\|\mathbf{u}_{i}\right\|_{2}^{2}+\mu\right)\left\{2 \frac{\left(b_{i}^{1 / 2}-b_{i-1}^{1 / 2}\right)}{\left(\frac{b_{i}-b_{i-1}}{\tau_{i}-\tau_{i-1}}\right)}\right\}=\sum_{i=1}^{N} \frac{2\left(\left\|\mathbf{u}_{i}\right\|_{2}^{2}+\mu\right)\left(\tau_{i}-\tau_{i-1}\right)}{\left(\sqrt{b_{i}}+\sqrt{b_{i-1}}\right)} .
\end{aligned}
$$

A dinâmica contínua do sistema descrita pela equação (3.33), se avaliada em $\bar{\tau}_{i}$, implica em:

$$
\mathbf{R}_{\mathbf{q}}\left(\bar{\tau}_{i}\right) \mathbf{u}\left(\bar{\tau}_{i}\right)=\mathbf{M}_{\mathbf{q}}\left[\mathbf{s}^{\prime}\left(\bar{\tau}_{i}\right) a\left(\bar{\tau}_{i}\right)+\mathbf{s}^{\prime \prime}\left(\bar{\tau}_{i}\right) b\left(\bar{\tau}_{i}\right)\right]
$$

Para discretizar a equação (3.48) se faz necessário a estimação discreta das derivadas do caminho s. Supõe-se que sejam apresentados no problema os valores de $\mathbf{s}$ em cada tempo discreto, ou seja, $\mathbf{s}_{i} \in \mathbb{R}^{2}, \mathbf{s}_{i}=\mathbf{s}\left(\tau_{i}\right)=\left[\gamma\left(\tau_{i}\right) \theta\left(\tau_{i}\right)\right]^{T}, i=0, \ldots, N$. Então, no ponto médio entre dois tempos discretos o valor de $s$ pode ser aproximado por

$$
\overline{\mathbf{s}}_{i}=\mathbf{s}\left(\bar{\tau}_{i}\right)=\frac{\mathbf{s}_{i}+\mathbf{s}_{i-1}}{2},
$$


$\overline{\mathbf{s}}_{i} \in \mathbb{R}^{2}, \overline{\mathbf{s}}_{i}=\left[\begin{array}{ll}\bar{\gamma}_{i} & \bar{\theta}_{i}\end{array}\right]^{T}$, e a derivada no ponto médio por

$$
\overline{\mathbf{s}}_{i}^{\prime}=\mathbf{s}^{\prime}\left(\bar{\tau}_{i}\right)=\frac{\mathbf{s}_{i}-\mathbf{s}_{i-1}}{\tau_{i}-\tau_{i-1}}
$$

$\overline{\mathbf{s}}_{i}^{\prime} \in \mathbb{R}^{2}, \overline{\mathbf{s}}_{i}^{\prime}=\left[\begin{array}{ll}\bar{\gamma}_{i}^{\prime} & \bar{\theta}_{i}^{\prime}\end{array}\right]^{T}, i=0, \ldots, N$

Para a segunda derivada do caminho, Lipp e Boyd (2014) sugerem uma aproximação baseada no método simétrico de Runge-Kutta de sexta ordem,

$$
\overline{\mathbf{s}}_{i}^{\prime \prime}=\mathbf{s}^{\prime \prime}\left(\bar{\tau}_{i}\right)=\frac{-\frac{5}{48} \mathbf{s}_{i-3}+\frac{13}{16} \mathbf{s}_{i-2}-\frac{17}{24} \mathbf{s}_{i-1}-\frac{17}{24} \mathbf{s}_{i}+\frac{13}{16} \mathbf{s}_{i+1}-\frac{5}{48} \mathbf{s}_{i+2}}{\left(\tau_{i}-\tau_{i-1}\right)^{2}},
$$

$\overline{\mathbf{s}}_{i}^{\prime \prime} \in \mathbb{R}^{2}, \overline{\mathbf{s}}_{i}^{\prime \prime}=\left[\begin{array}{ll}\bar{\gamma}_{i}^{\prime \prime} & \bar{\theta}_{i}^{\prime \prime}\end{array}\right]^{T}, i=0, \ldots, N$.

Como $i=1 \ldots N$, a equação (3.51) não pode ser avaliada diretamente em $\bar{\tau}_{1}, \bar{\tau}_{2}$, $\bar{\tau}_{N-1}$ e $\bar{\tau}_{N}$. Para tanto, é necessário estimar pontos anteriores ao tempo inicial e posteriores ao tempo final.

Propõe-se avaliar $\mathbf{s}^{\prime \prime}\left(\bar{\tau}_{i}\right)$ através do método de Runge-Kutta de sexta ordem para $i=2, \ldots, N-2$ aplicando a Equação (3.51), e o método Runge-Kutta de quarta ordem para os extremos $i=\{1,2, N-1, N\}$.

Assume-se, para tanto, que o caminho $\mathbf{s}(\tau)$ é linear no início e no fim do caminho. Neste sentido, estima-se que $\mathbf{s}_{-1}=2 \mathbf{s}_{0}-\mathbf{s}_{1}$ e $\mathbf{s}_{N+1}=2 \mathbf{s}_{N}-\mathbf{s}_{N-1}$. Pela equação de Runge-Kutta de quarta ordem,

$$
\overline{\mathbf{s}}_{i}^{\prime \prime}=\frac{\mathbf{s}_{i-2}-\mathbf{s}_{i-1}-\mathbf{s}_{i}+\mathbf{s}_{i+1}}{2\left(\tau_{i}-\tau_{i-1}\right)^{2}}
$$

tem-se:

$$
\begin{aligned}
\overline{\mathbf{s}}_{1}^{\prime \prime} & =\frac{\mathbf{s}_{0}-2 \mathbf{s}_{1}+\mathbf{s}_{2}}{2\left(\tau_{1}-\tau_{0}\right)^{2}}, & \overline{\mathbf{s}}_{2}^{\prime \prime} & =\frac{\mathbf{s}_{0}-\mathbf{s}_{1}-\mathbf{s}_{2}+\mathbf{s}_{3}}{2\left(\tau_{2}-\tau_{1}\right)^{2}}, \\
\overline{\mathbf{s}}_{(N-1)}^{\prime \prime} & =\frac{\mathbf{s}_{(N-3)}-\mathbf{s}_{(N-2)}-\mathbf{s}_{(N-1)}+\mathbf{s}_{(N)}}{2\left(\tau_{(N-1)}-\tau_{(N-2)}\right)^{2}}, & \overline{\mathbf{s}}_{N}^{\prime \prime} & =\frac{\mathbf{s}_{(N-2)}-\mathbf{s}_{(N-1)}+\mathbf{s}_{N}}{2\left(\tau_{N}-\tau_{(N-1)}\right)^{2}} .
\end{aligned}
$$

Então, considerando as equações (3.44), (3.47) e (3.48), a discretização do problema 3.2 pode ser escrito da forma:

Problema 3.3. (Problema discreto de mínimo Tempo-Energia) 


$$
\begin{aligned}
\underset{\left(\mathbf{u}_{i}, a_{i}, b_{i}\right)}{\operatorname{minimize}}: & \mathcal{J}(\tau, \mathbf{u})=\sum_{i=1}^{N} \frac{2\left(\left\|\mathbf{u}_{i}\right\|_{2}^{2}+\mu\right)}{\left(\sqrt{b_{i}}+\sqrt{b_{i-1}}\right)}\left(\delta \tau_{i}\right) \\
\text { sujeito a : } \quad \mathbf{R}_{\mathbf{q}} \mathbf{u}_{i} & =\mathbf{M}_{\mathbf{q}}\left[\overline{\mathbf{s}}_{i}^{\prime} a_{i}+\frac{\overline{\mathbf{s}}_{i}^{\prime \prime}}{2} b_{i}+\frac{\overline{\mathbf{s}}_{i}^{\prime \prime}}{2} b_{i-1}\right] \\
b_{i}-b_{i-1} & =2 a_{i}\left(\delta \tau_{i}\right) \\
\left(\mathbf{u}_{i}, a_{i}, b_{i}\right) & \in \mathcal{C}_{\tau}, \quad i=1, \ldots, N,
\end{aligned}
$$

onde $\mathcal{C}_{\tau} \subseteq \mathbb{R}^{2 x 2 x 2}$ apresentado como restrição na Equação 3.56 , dado pelo conjunto $\mathcal{C}_{\tau}=$ $\left\{(\mathbf{u}(\tau), a(\tau), b(\tau)) \mid\left(\mathbf{u}(\tau), \mathbf{s}^{\prime 2}(\tau) b(\tau), \mathbf{s}^{\prime}(\tau) a(\tau)+\mathbf{s}^{\prime \prime}(\tau) b(\tau)\right) \in \mathcal{C}_{t}\right\}$, é o conjunto convexo que limita o domínio do problema, e $\delta \tau_{i}=\left(\tau_{i}-\tau_{i-1}\right)$.

O Problema 3.3 é um problema de otimização convexa, pois suas equações foram discretizadas a partir de um problema convexo. Apesar da função objetivo $\mathcal{J}(\tau, u)$ ser uma função convexa, ela é não-linear.

Além disso, seus termos são da forma $F_{a} / F_{b}$, onde $F_{a}$ é uma função convexa e $F_{b}$ é uma função côncava em seus domínios. Apesar de $F_{a} / F_{b}$ ser uma função convexa (ver subseção 3.2.1), o pacote de otimização CVX para Matlab ${ }^{\circledR}$ (GRANT; BOYD, 2014) exige uma função objetivo "disciplinada", ou seja, possua seus termos tais que o pacote possa avaliar a convexidade. Como nem toda função da forma convex/concave é convexa, o pacote de otimização não é capaz de realizar sua tarefa.

Para resolver este problema com o pacote CVX, propõe-se reformular o problema de otimização como uma Programação Cônica de Segunda Ordem, apresentada na seção seguinte.

\subsection{FORMULAÇÃO COMO PROGRAMAÇÃO CÔNICA DE SEGUNDA OR- DEM - SOCP}

Um problema de Programação Cônica de Segunda Ordem (Second Order Cone Programming - SOCP) tem como forma padrão (BOYD; VANDENBERGHE, 2004):

$$
\begin{aligned}
\text { minimize } & f^{T} x \\
\text { sujeito a } & \left\|A_{i} x+b_{i}\right\|_{2} \leq c_{i}^{T} x+d_{i}, \quad i=1, \ldots, m \\
& F x=g,
\end{aligned}
$$

onde $x \in \mathbb{R}^{n}$ e a variável de otimização, $A_{i} \in \mathbb{R}^{n_{i} \times n}$, e $F \in \mathbb{R}^{p \times n}$. Uma restrição $\|A x+b\|_{2} \leq$ $c^{T} x+d$, onde $A \in \mathbb{R}^{k \times n}$, é chamada de restrição cônica de segunda ordem, devido ao fato de que ela impõe que as funções afins $\left(A x+b, c^{T} x+d\right)$ pertençam a um cone de segunda ordem em $\mathbb{R}^{k+1}$. 
Este tipo de problema é convexo pois, além da função objetivo (3.57) e da restrição de igualdade (3.59) serem lineares, a função $\left\|A_{i} x+b_{i}\right\|_{2}-c_{i}^{T} x-d_{i} \leq 0$ é uma função afim de uma norma e, portanto, convexa.

Introduz-se no Problema 3.3 quatro novas variáveis $c_{i}, d_{i}, e_{i}, f_{i} \in \mathbb{R}, c_{i}>0, d_{i}>0$, $e_{i}>0, f_{i}>0, i=1, \ldots, N$, tal que a função objetivo (3.53) possa ser redefinida como

$$
\begin{array}{rlrl}
\mathcal{J}(\tau, \mathbf{u}) & =\overbrace{\sum_{i=1}^{N} \frac{2\left(\mathbf{u}_{i}^{T} \mathbf{u}_{i}\right)}{\left(\sqrt{b_{i}}+\sqrt{b_{i-1}}\right)}\left(\delta \tau_{i}\right)}^{\text {energia }} & +\overbrace{\sum_{i=1}^{N} \frac{2}{\left(\sqrt{b_{i}}+\sqrt{b_{i-1}}\right)}\left(\delta \tau_{i}\right)}^{\text {tempo }} \leq \\
& \leq \sum_{i=1}^{N} \frac{2\left(\mathbf{u}_{i}^{T} \mathbf{u}_{i}\right)}{\left(c_{i}+c_{i-1}\right)}\left(\delta \tau_{i}\right) & +\mu \sum_{i=1}^{N} \frac{2}{\left(c_{i}+c_{i-1}\right)}\left(\delta \tau_{i}\right) & \leq \\
& \leq \sum_{i=1}^{N} \frac{2\left(\mathbf{u}_{i}^{T} \mathbf{u}_{i}\right)}{d_{i}}\left(\delta \tau_{i}\right) & +\mu \sum_{i=1}^{N} \frac{2}{d_{i}}\left(\delta \tau_{i}\right) & \leq \\
& \leq \sum_{i=1}^{N} 2 e_{i}\left(\delta \tau_{i}\right) & +\mu \sum_{i=1}^{N} 2 f_{i}\left(\delta \tau_{i}\right) & =\tilde{J}(\tau, \mathbf{u}),
\end{array}
$$

onde as desigualdades

$$
\begin{aligned}
\frac{1}{d_{i}} & \leq f_{i}, \\
\frac{\mathbf{u}_{i}^{T} \mathbf{u}_{i}}{d_{i}} & \leq e_{i}, \\
d_{i} & \leq c_{i}+c_{i-1}, \\
c_{i} & \leq \sqrt{b_{i}},
\end{aligned}
$$

possam ser consideradas restrições que definam a função objetivo $\tilde{J}(\tau, \mathbf{u})$ como barreira imposta para a função $J(\tau, \mathbf{u})$.

Teorema 3.2 (Restrições hiperbólicas como restrições cônicas de segunda ordem). Para $w, x, y \in \mathbb{R} . x \geq 0, y \geq 0$, tem-se (LOBO et al., 1998):

$$
w^{2} \leq x y \Longleftrightarrow\left\|\left[\begin{array}{c}
2 w \\
x-y
\end{array}\right]\right\|_{2} \leq x+y,
$$

e, mais genericamente, quando $w \in \mathbb{R}^{n}$, tem-se:

$$
w^{T} w \leq x y \Longleftrightarrow\left\|\left[\begin{array}{c}
2 w \\
x-y
\end{array}\right]\right\|_{2} \leq x+y .
$$

Aplicando-se o Teorema 3.2 nas desigualdades (3.61), (3.62) e (3.64), tem-se: 


$$
\begin{aligned}
& \left\|\left[\begin{array}{c}
2 \\
d_{i}-f_{i}
\end{array}\right]\right\|_{2} \leq d_{i}+f_{i} \\
& \left\|\left[\begin{array}{c}
2 u_{i} \\
e_{i}-d_{i}
\end{array}\right]\right\|_{2} \leq e_{i}+d_{i} \\
& \left\|\left[\begin{array}{c}
2 c_{i} \\
b_{i}-1
\end{array}\right]\right\|_{2} \leq b_{i}+1 .
\end{aligned}
$$

Portanto, o problema 3.3 é equivalente ao seguinte problema:

Problema 3.4. (Problema discreto de mínimo Tempo-Energia na forma SOCP)

$$
\begin{aligned}
& \underset{\left(\mathbf{u}_{i}, a_{i}, b_{i}, c_{i}, d_{i}, e_{i}, f_{i}\right)}{\operatorname{minimize}}: \tilde{\mathcal{J}}(\tau, \mathbf{u})=\sum_{i=1}^{N} 2\left(e_{i}+\mu f_{i}\right) \delta \tau_{i} \\
& \text { sujeito } a: \mathbf{R}_{\mathbf{q}} \mathbf{u}_{i}=\mathbf{M}_{\mathbf{q}}\left[\overline{\mathbf{s}}_{i}^{\prime} a_{i}+\frac{\overline{\mathbf{s}}_{i}^{\prime \prime}}{2} b_{i}+\frac{\overline{\mathbf{s}}_{i}^{\prime \prime}}{2} b_{i-1}\right] \\
& b_{i}-b_{i-1}=2 a_{i}\left(\delta \tau_{i}\right) \\
& d_{i} \leq c_{i}+c_{i-1} \\
&\left\|\left[\begin{array}{c}
2 \\
d_{i}-f_{i}
\end{array}\right]\right\|_{2} \leq d_{i}+f_{i} \\
&\left\|\left[\begin{array}{c}
2 u_{i} \\
e_{i}-d_{i}
\end{array}\right]\right\|_{2} \leq e_{i}+d_{i} \\
&\left\|\left[\begin{array}{c}
2 c_{i} \\
b_{i}-1
\end{array}\right]\right\|_{2} \leq b_{i}+1 \\
&\left(\mathbf{u}_{i}, a_{i}, b_{i}\right) \in \mathcal{C}_{\tau}, \quad i=1, \ldots, N,
\end{aligned}
$$

onde $\mathcal{C}_{\tau} \subseteq \mathbb{R}^{2 x 3 x 3}$ dado como restrição na Equação 3.75 é o conjunto convexo $\mathcal{C}_{\tau}=$ $\left\{(\mathbf{u}(\tau), a(\tau), b(\tau)) \mid\left(\mathbf{u}(\tau), \mathbf{s}^{\prime 2}(\tau) b(\tau), \mathbf{s}^{\prime}(\tau) a(\tau)+\mathbf{s}^{\prime \prime}(\tau) b(\tau)\right) \in \mathcal{C}_{t}\right\}$

Como resultado do Problema 3.4 tem se a entrada em tensão ótima $\mathbf{u}^{*}\left(\bar{\tau}_{i}\right)=$ $\left[u_{r}^{*}\left(\bar{\tau}_{i}\right) u_{l}^{*}\left(\bar{\tau}_{i}\right)\right]^{T}$. A reparametrização temporal pode ser obtida pela relação entre o tempo $t$ e o tempo virtual $\tau$

$$
\delta t_{i}=\frac{\delta \tau_{i}}{\sqrt{b_{i}^{*}}}
$$

onde $b^{*}$ é o parâmetro $b$ otimizado pelo algorítmo. O tempo final de percurso do robô na curva $\Gamma$ dado por $T_{f}$ é o somatório

$$
T_{f}=\sum_{1}^{N} \frac{\delta \tau_{i}}{\sqrt{b_{i}^{*}}} .
$$


A energia total gasta pelo robô em seu percurso, $E_{t}$, é dada por

$$
E_{t}=\sum_{1}^{N}\left\|\mathbf{u}_{i}^{*}\right\|_{2}^{2} \delta \tau_{i},
$$

cuja unidade é dada em Joule $(\mathrm{J})$ no Sistema Internacional de Unidades - SI, equivalente a $\left[V^{2} s / \Omega\right] .{ }^{1}$ A velocidade linear do robô em cada intervalo de tempo $\left[\tau_{i-1}, \tau_{i}\right], i=1, \ldots, N$ é dada por

$$
v(\bar{\tau})=v_{i}=\frac{\gamma_{i}^{\prime} \sqrt{b_{i}^{*}}}{\delta \tau_{i}}
$$

\subsection{RESULTADOS NUMÉRICOS DO PLANEJAMENTO ÓTIMO}

O Problema 3.4 está formulado como uma Programação Cônica de Segunda Ordem, com variáveis de otimização o sinal de controle em tensão elétrica $\mathbf{u}_{i}$, os vetores de aceleração e do quadrado da velocidade representados pelos termos $a_{i}$ e $b_{i}$ respectivamente, além de variáveis auxiliares para a linearização da função objetivo $\tilde{\mathcal{J}}$ dados pelos vetores $c, d, e, f \in \mathbb{R}^{N}$.

Seja então um robô uniciclo diferencial, como definido na Seção 3.1, de largura $B=0,4 m$, com uma massa total de $m=10 \mathrm{~kg}$ e que cujas características construtivas o conferem um momento de inércia de $J=2,833 \mathrm{Kg} / \mathrm{m}^{2}$. Suas rodas possuem um raio de $r=0,1 \mathrm{~m}$ que são acionadas por motores elétricos independentes cuja constante de torque é de $K_{m}=65 \cdot 10^{-3} \mathrm{Nm} / \mathrm{V}$ e cuja tensão nominal é de $u_{\max }=-u_{\min }=12 \mathrm{~V}$ para cada roda.

\subsubsection{Geração de dados}

Supõe-se que uma camada hierárquica superior seja responsável por gerar um caminho geométrico por onde o robô deva percorrer. Para tanto, dado um conjunto de pontos de controle (ou waypoints), esta camada superior interpola uma curva $\Gamma$ definida por uma função $\mathbf{p}(\tau)=[x(\tau) \quad y(\tau)]^{T}, \tau=[0,1]$, discretizada em $N+1$ pontos definidos por $\mathbf{p}_{i}=\left[\begin{array}{ll}x_{i} & y_{i}\end{array}\right]^{T}, i=0, \ldots, N$.

Como exemplo, seja então uma curva $\Gamma$ ilustrada na Figura 18, com $N=200$. Esta curva hipotética é gerada por um algoritmo que interpola pontos entre os waypoints: $w p_{0}=\left[\begin{array}{ll}0 & 0\end{array}\right]^{T}, w p_{1}=\left[\begin{array}{ll}2 & -1\end{array}\right]^{T}, w p_{2}=\left[\begin{array}{ll}3 & 3\end{array}\right]^{T}, w p_{3}=\left[\begin{array}{ll}6 & 3\end{array}\right]^{T}, w p_{4}=\left[\begin{array}{ll}8 & 0\end{array}\right]^{T} \mathrm{e}$ $w p_{5}=\left[\begin{array}{ll}5 & -2\end{array}\right]^{T}$.

Cada ponto discreto da curva $\Gamma$, dado pela camada hierárquica superior na forma $\mathbf{p}_{i}=\left[\begin{array}{ll}x_{i} & y_{i}\end{array}\right]^{T}, i=0, \ldots, N$, pode ser representado na configuração generalizada alternativa $\mathbf{s}_{i}=\left[\begin{array}{ll}\gamma_{i} & \theta_{i}\end{array}\right]^{T}, i=0, \ldots, N$ através das transformações (3.9) e (3.10) em sua forma discreta. Esta transformação é apresentada no Algoritmo 3 e no Apêndice B.1.

1 Considera-se a impedância dos motores como linear e invariante no tempo $Z=1 \Omega$. 


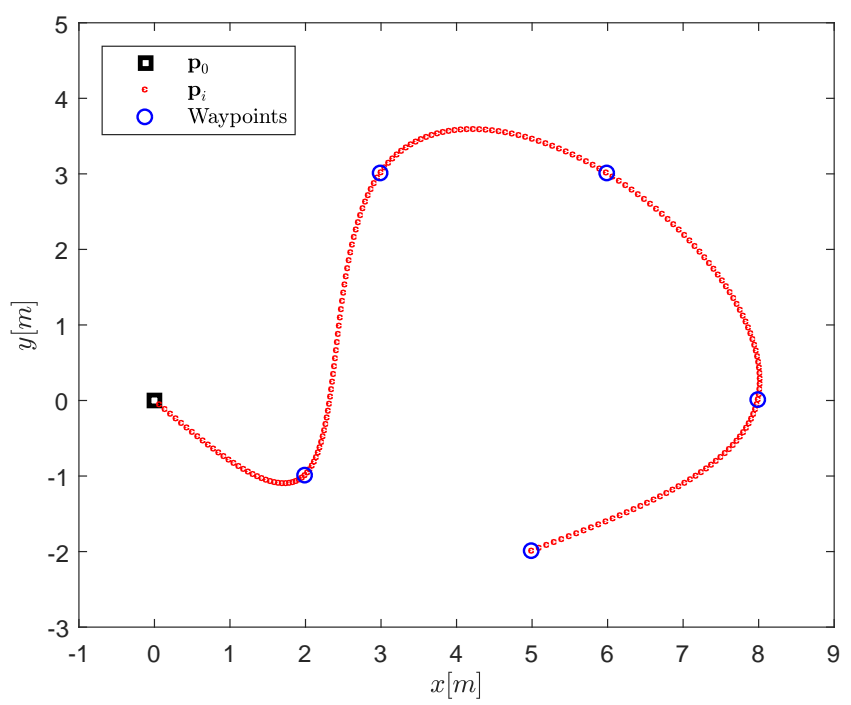

Figura 18 - Gráfico de uma curva $\Gamma$ no plano cartesiano. O quadrado representa a posição inicial $\mathbf{p}_{0}$, os círculos representam os waypoints que geram a curva, e os pontos representam as posições $\mathbf{p}_{i}, i=1, \ldots, N=200$.

Fonte: o autor.

A partir dos pontos discretos representados por $\mathbf{s}_{i}, i=0, \ldots, N$, são estimados os pontos médios $\overline{\mathbf{s}}_{i}$ pela Equação 3.49, suas derivadas de primeira ordem $\overline{\mathbf{s}}_{i}^{\prime}$ pela Equação 3.50 e suas derivadas de segunda ordem $\overline{\mathbf{s}}_{i}^{\prime \prime}$ pelas Equações 3.51 e 3.52. Os resultados destas estimações são ilustrados na Figura 19.

Para avaliar a estimação das derivadas de segunda ordem nos pontos médios $\overline{\mathbf{s}}_{i}^{\prime \prime}$, são realizados somatórios integrativos de seus valores por duas vezes consecutivas. Como resultado, têm-se pontos $\overline{\mathbf{s}}_{i}=\left[\begin{array}{ll}\bar{\gamma}_{i} & \bar{\theta}_{i}\end{array}\right]^{T}$ estimados.

O erro de estimação é sensível ao número de interpolantes na curva $N$. Quanto maior o valor de $N$, menor é o diferencial $\delta \tau_{i}=\tau_{i}-\tau_{i-1}$ e, portanto, mais precisa a diferenciação pelos métodos de Runge-Kutta, como pode ser observado nos gráficos da Figura 20.

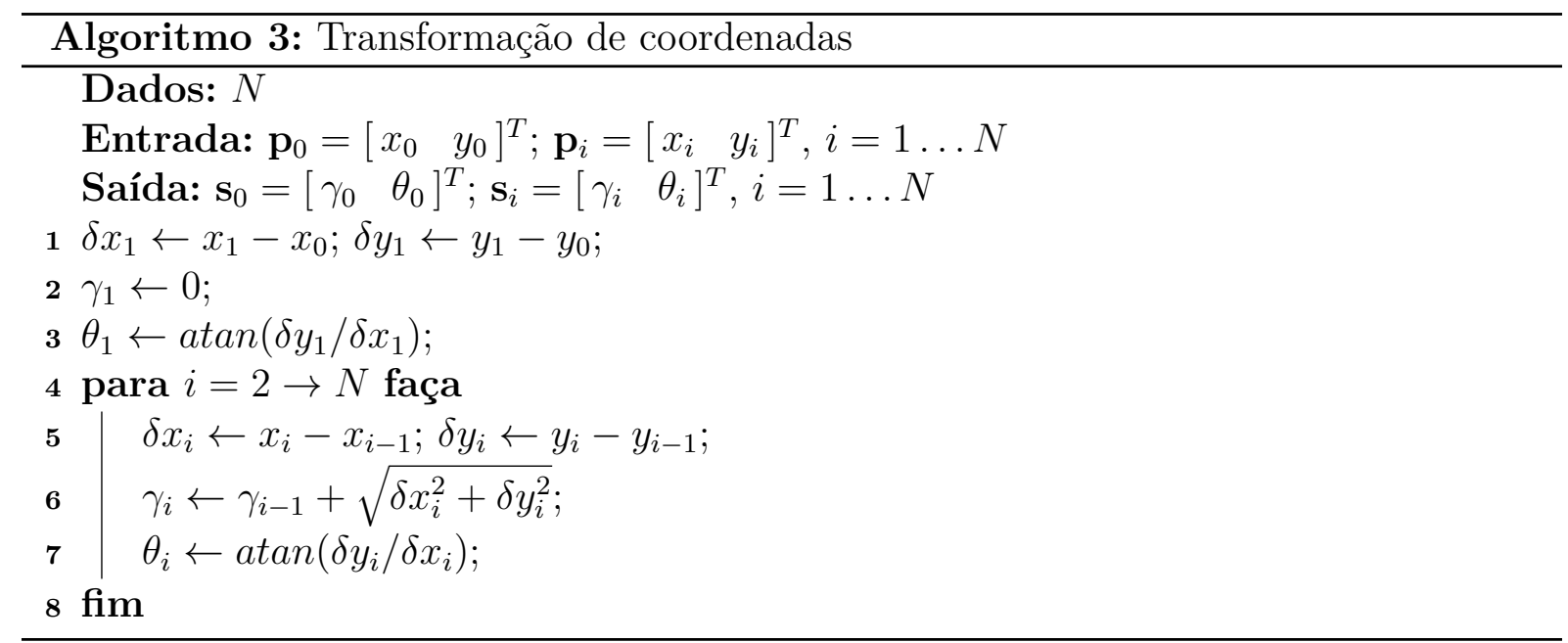



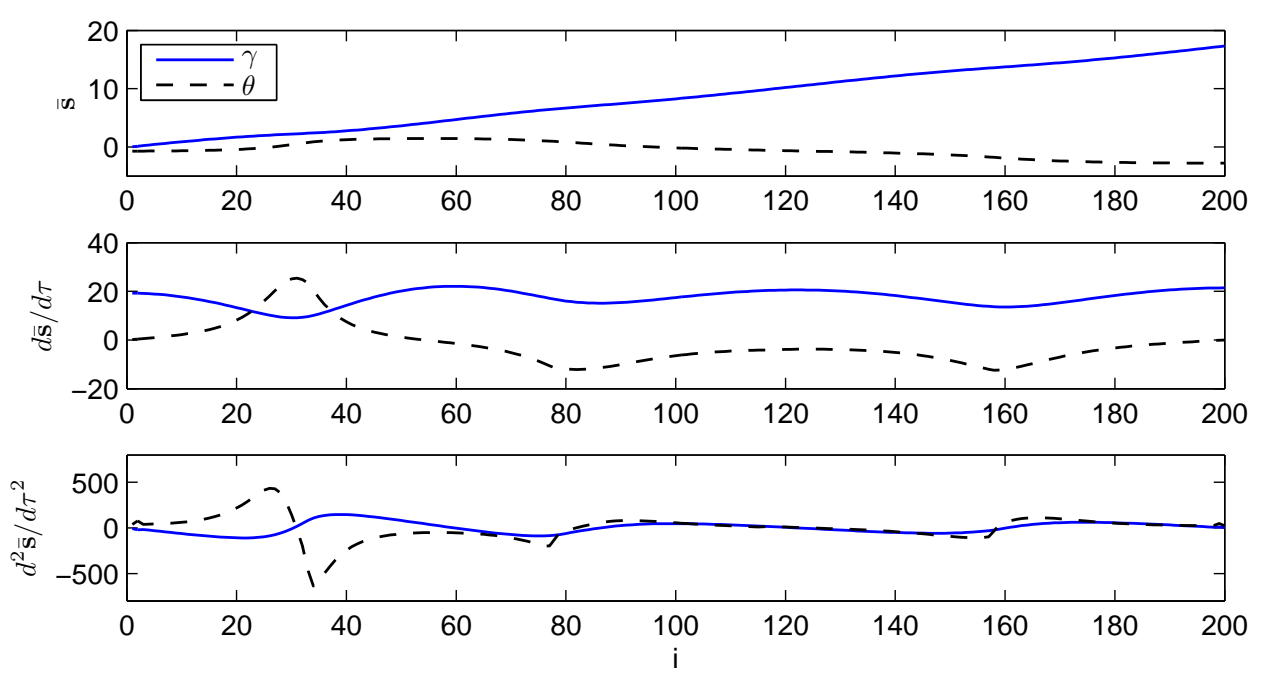

Figura 19 - Gráfico dos componentes do caminho $\Gamma$ medidos no tempo médio discreto $\overline{\mathbf{s}}_{i}$ e suas derivadas de primeira e segunda ordem. Em azul contínuo as curvas referentes ao componente $\gamma$, e em preto tracejado ao componente $\theta$.

Fonte: o autor.
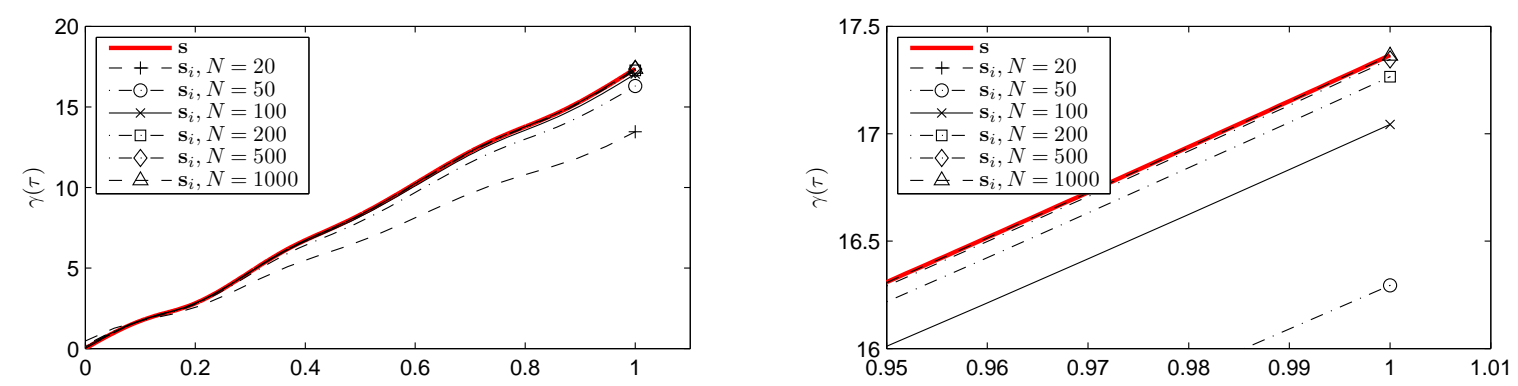

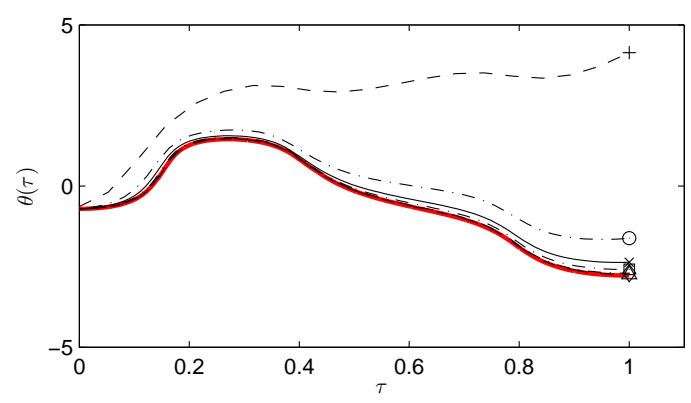

(a) Gráfico em todo o caminho

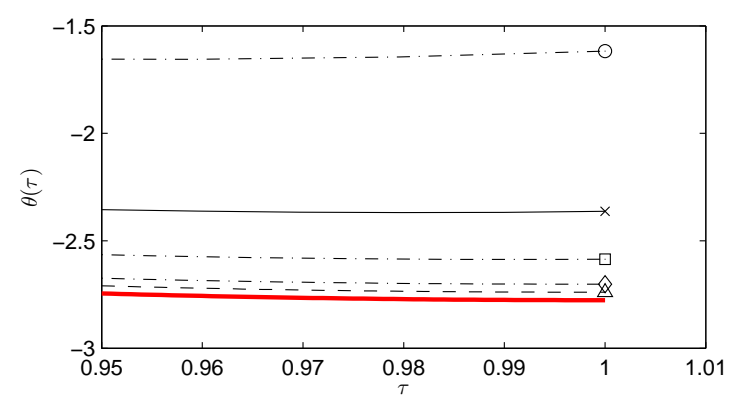

(b) Detalhe no final do caminho

Figura 20 - Gráfico dos componentes do caminho $\Gamma$ gerados pela estimação das derivadas de segunda ordem pelo método de Runge-Kutta. A linha vermelha espessa ilustra o valor original dos componentes $\gamma$ (nos gráficos superiores) e $\theta$ (nos gráficos inferiores), enquanto as linhas em preto ilustram a estimação dos componentes com a variação de $N$.

Fonte: o autor. 
Através das trasformações dadas pelas equações 3.12, estas estimativas podem ser representadas como pontos no plano cartesiano, como ilustra a Figura 21. No plano cartesiano fica mais evidente que, quanto maior a quantidade de interpolantes da curva, mais precisa é a diferenciação dos componentes da representação em $\mathbf{s}$ da curva $\Gamma$.

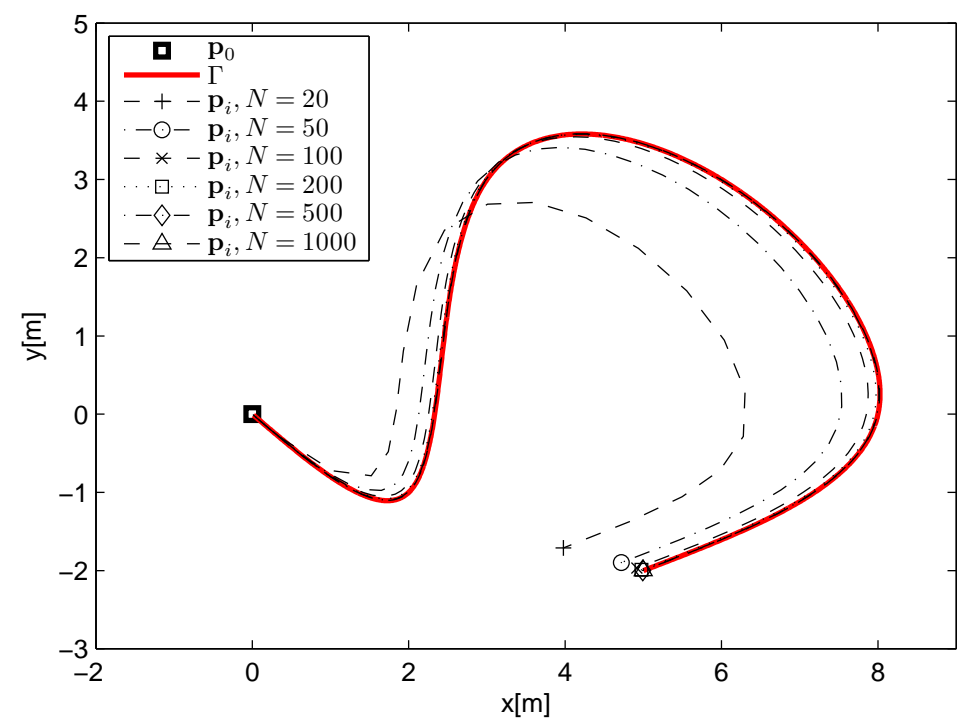

Figura 21 - Gráfico dos pontos no plano cartesiano gerados pela estimação das derivadas de segunda ordem pelo método de Runge-Kutta. A linha vermelha espessa ilustra o valor original da curva $\Gamma$, enquanto as linhas em preto ilustram a estimação do caminho com a variação de $N$.

Fonte: o autor.

Para se avaliar quantitativamente esta estimação, foram calculados os erros médios de posição entre os pontos $\mathbf{p}_{i}$ da curva original $\Gamma$ discretizada e as curvas ilustradas na Figura 21. Os resultados são apresentados na Tabela 1, e representam o quão precisa é a estimativa das derivadas de $s$ pelo método de diferenciação por Runge-Kutta em relação ao número de interpolantes $N$.

Tabela 1 - Erro médio de posição da estimativa por Runge-Kutta.

\begin{tabular}{ll}
\hline $\mathrm{N}$ & Erro Médio $[\mathrm{m}]$ \\
\hline 20 & 1,1205 \\
50 & 0,3338 \\
100 & 0,1108 \\
200 & 0,0404 \\
500 & 0,0125 \\
1000 & 0,0057 \\
\hline \multicolumn{3}{c}{ Fonte: o autor }
\end{tabular}

\subsubsection{Otimização}

O Problema 3.4 é transformado em código para ser executado como Programação Cônica de Segunda Ordem - SOCP (Apêndice B.2). 
Todas as simulações realizadas neste trabalho foram executadas utilizando o Matlab $^{\circledR}$ R2017a versão estudante, com o pacote CVX (GRANT; BOYD, 2014) na sua versão 2.1. As simulações foram executadas em um computador com processador Intel Core I7-4790, frequência de 3.6GHz de clock, 8GB de memória RAM e sistema operacional Windows ${ }^{\circledR} 8.1$.

Como primeira ilustração, seja então a curva $\Gamma$ até então apresentada como exemplo, discretizada em $N=200$ pontos. Como restrições ao problema de otimização são impostas $u_{\max }=-u_{\min }=\left[\begin{array}{lll}12 \mathrm{~V} & 12 \mathrm{~V}\end{array}\right]^{T} ; \dot{\mathbf{q}}_{\max }=-\dot{\mathbf{q}}_{\min }=\left[\begin{array}{ll}2,5 \mathrm{~m} / \mathrm{s} & 1 \mathrm{rad} / \mathrm{s}\end{array}\right]^{T}$ e $\ddot{\mathbf{q}}_{\max }=-\ddot{\mathbf{q}}_{\min }=$ $\left[2 \mathrm{~m} / \mathrm{s}^{2} \quad 0,5 \mathrm{rad} / \mathrm{s}^{2}\right]^{T}$.

O algoritmo de otimização usado para esta ilustração inicial é o SDPT3 que, em $0,52 s$ de tempo de processamento, teve como resultado as tensões ótimas $u_{r}^{*}(t)$ e $u_{l}^{*}(t)$ (dos motores direito e esquerdo, respectivamente) e o perfil da velocidade linear $v^{*}(t)$ ilustrados na Figura 22

O coeficiente de ponderação tempo-energia usado nesta otimização foi de $\mu=100$. Nestas condições, o robô percorre o caminho dado $\Gamma$ em um tempo de $T_{f}=16,2 s$, consumindo uma energia de $E_{t}=463,8 \mathrm{~J}$.
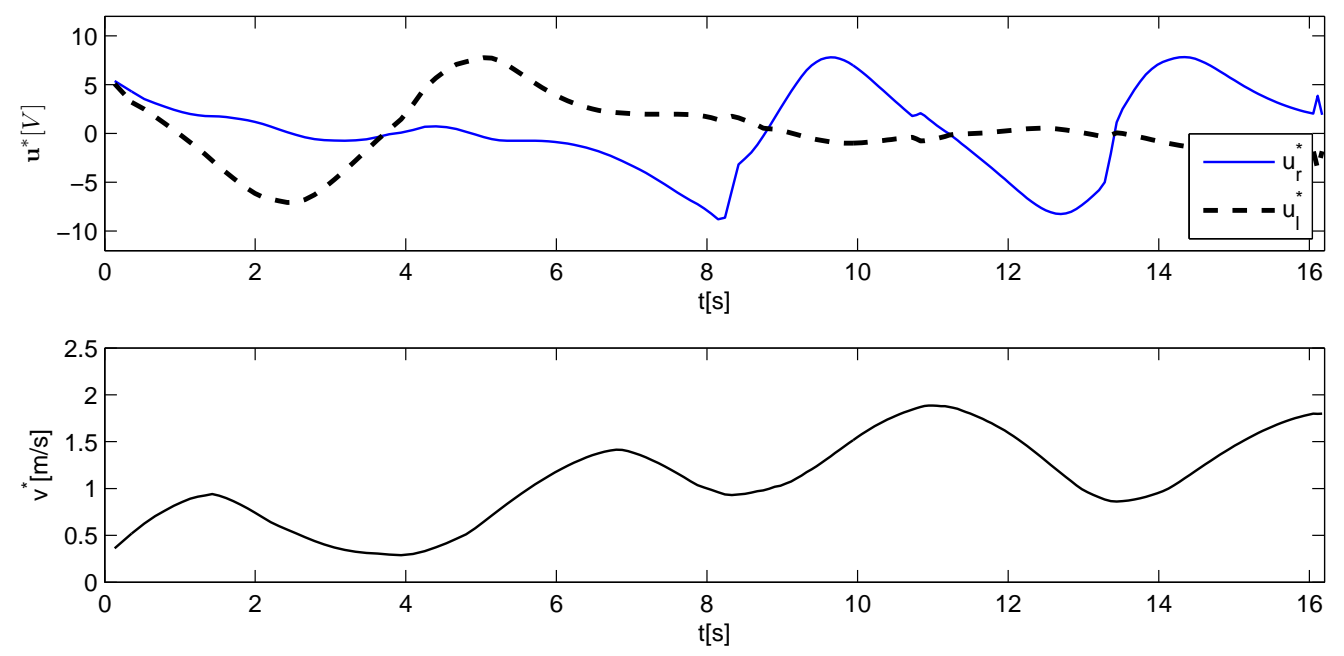

Figura 22 - Gráficos de resposta da otimização para $\mu=100, N=200$. No gráfico superior, a tensão do motor direito em linha contínua, e a tensão do motor esquerdo em linha tracejada. No gráfico inferior, a velocidade linear do robô.

Fonte: o autor.

O pacote CVX, em sua versão profissional, inclui quatro algoritmos de otimização: (i) SDPT3, (ii) SeDuMi, (iii) Mosek e (iv) Gurobi. Para avaliar a utilização de cada um destes algoritmos no problema de planejamento de trajetória, foi realizada uma série de otimizações variando o número de discretização $N$, seguindo uma série da forma $N=[10,20, \ldots, 100,200, \ldots, 1000,2000, \ldots, 10000]$.

Esta série de otimizações foi realizada nas mesmas condições da primeira ilustração 
- tanto no que se diz respeito ao coeficiente de ponderação $(\mu=100)$ quanto às restrições de velocidade e de aceleração ( $\dot{\mathbf{q}}$ e $\ddot{\mathbf{q}})$.

\subsubsection{Quanto ao tempo de processamento}

O tempo de processamento do algoritmo de otimização está intimamente ligado à quantidade de variáveis de otimização e de restrições. O problema de otimização (Problema 3.4) possui $29 N-1$ variáveis escalares (a função objetivo, as 14 variáveis do problema primário e 14 do dual, com excessão de $b_{0}$ ), $11 N-1$ restrições lineares (equações 3.69, 3.70 e 3.75 com excessão de $b_{0}$ ), e $3 N$ restrições quadráticas (equações 3.72, 3.72 e 3.72).

Não se percebe diferença significativa no tempo de processamento entre os quatro algoritmos de otimização utilizados (conforme ilustrado na Figura 23) que justifique, por este critério, a escolha de algum algoritmo em específico. Cabe destacar que, apesar disso, o Mosek demonstrou-se ser um pouco mais rápido do que os outros algoritmos em toda a série de experimentação.

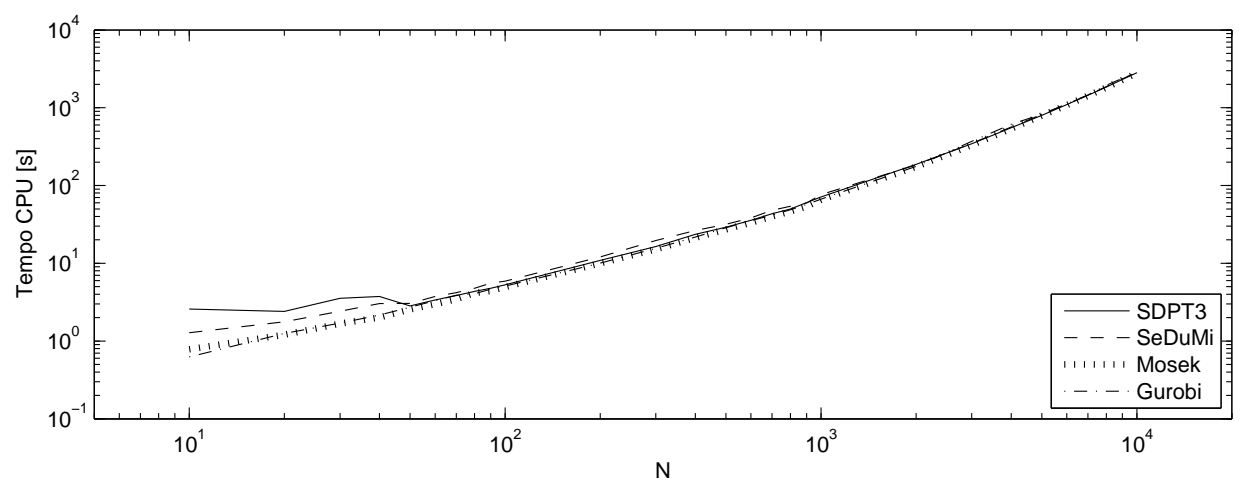

Figura 23 - Tempo de processamento versus número de discretização $N$ para os diferentes algoritmos de otimização.

Fonte: o autor.

\subsubsection{Quanto ao status final de processamento}

Os algoritmos de otimização executam até substituírem as variáveis do modelo pelos valores numéricos calculados e retornam o resultado da otimização na forma de uma string chamada cvx_status. Alguns dos valores possíveis para esta string e encontrados nas otimizações realizadas são (GRANT; BOYD, 2014):

- Resolvido (Solved): uma solução complementar (primário e dual) foi encontrada e sua diferença se encontra dentro da tolerância de parada do algoritmo $\left(\epsilon_{\text {standard }}\right)$. As variáveis são substituidas pelos seus valores computados, e o valor da função objetivo $\tilde{\mathcal{J}}$ do problema é registrado na variável cvx_optval.

- Impreciso/Resolvido (Inaccurate/Solved): indica que o algoritmo foi incapaz de fazer a determinação da solução complementar dentro da uma tolerância numérica 
padrão $\left(\epsilon_{\text {standard }}\right)$, mas dentro de uma tolerância "relaxada" $\left(\epsilon_{\text {reduced }}\right)$ e, portanto, podem ser utilizados como resultados numéricos..

- Falhado (Failed): o algoritmo não conseguiu fazer progressos suficientes em direção a uma solução, mesmo dentro da configuração de tolerância relaxada $\left(\epsilon_{\text {reduced }}\right)$. A variável cvx_optval é preenchida com o valor NaN ("Not-a-Number").

Os gráficos da Figura 24 ilustram as informações da variável cvx_status para os quatro algoritmos de otimização. Observa-se que tanto o algoritmo SDPT3 quanto o algoritmo SeDuMi só conseguem resolver esta otimização para um número de pontos discretos $N \leq 200$. Para modelos com mais de 6000 variáveis, o progresso da otimização é muito lento e o algoritmo decide fazer uma parada com status de falha.

Por outro lado, tanto os algoritmos Mosek quanto Gurobi apresentam boa convergência para uma solução dentro da tolerância relaxada, com especial destaque para o Mozek (Figura 24c), que convergiu para a solução padrão do algoritmo em todas as otimizações realizadas nesta série.

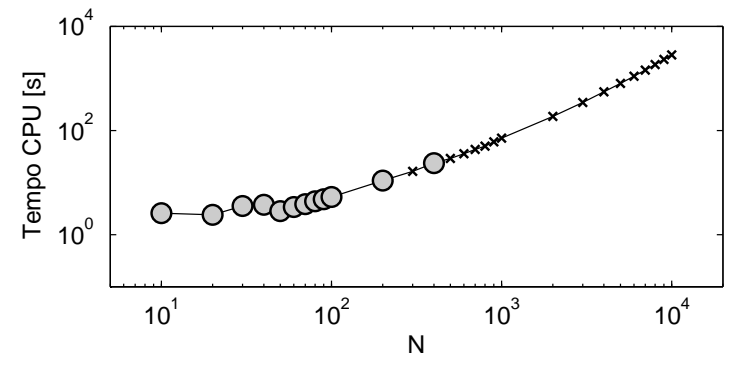

(a) SDPT3

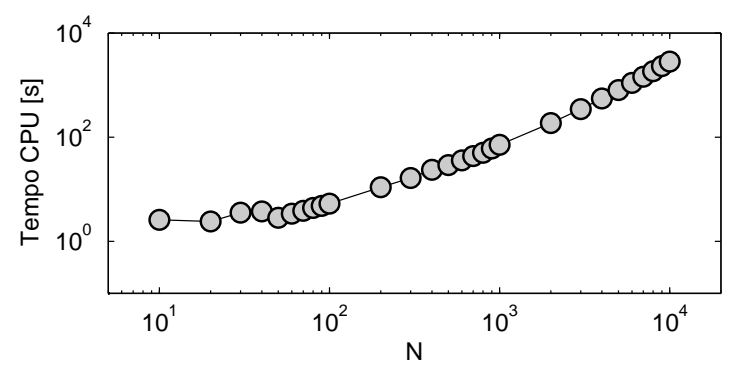

(c) Mosek

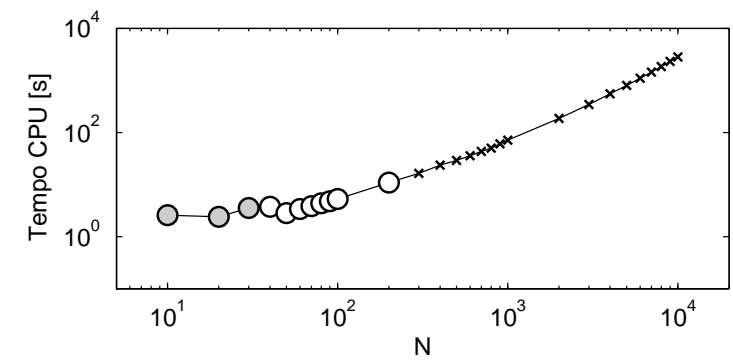

(b) SeDuMi

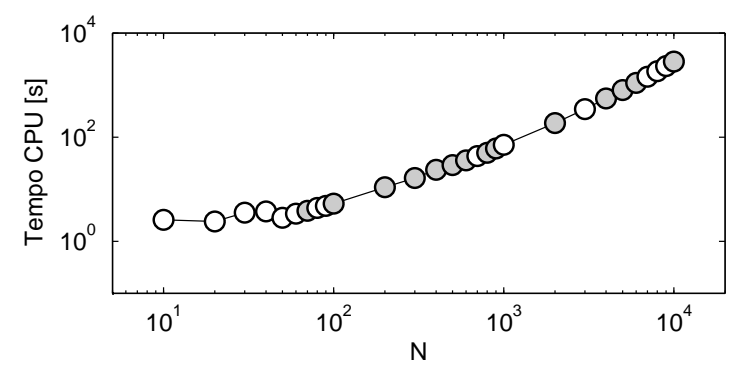

(d) Gurobi

Figura 24 - Status final da otimização versus número de discretização $N$ para os diferentes algoritmos. O círculo preenchido representa o status Resolvido, o círculo vazado o status Impreciso e as linhas cruzadas o status Falhado.

Fonte: o autor.

Para compreender qual o impacto de uma otimização com o status Impreciso em relação a uma com o status Resolvido no resultado final, os sinais de controle em tensão ótima dos motores $\left(u_{r}^{*}(t)\right.$ e $\left.u_{l}^{*}(t)\right)$ e de velocidade linear ótima $\left(v^{*}(t)\right)$ são comparados graficamente na Figura 25. 
Para $N=200$, os algoritmos SDPT3, Mosek e Gurobi apresentam resultados dentro da tolerância padrão (status Resolvido), enquanto o algoritmo SeDuMi resolve a otimização, porém fora da tolerância padrão, mas dentro da tolerância relaxada (status Impreciso).

Percebe-se graficamente que em termos qualitativos, os sinais ótimos de uma otimização imprecisa são muito parecidos com os sinais ótimos de uma otimização resolvida. Quantitativamente, a diferença máxima entre eles é de $7,98 \%$ para $u_{r}^{*}(t), 1,41 \%$ para $u_{l}^{*}(t)$ e $0,05 \%$ para $v^{*}(t)$.

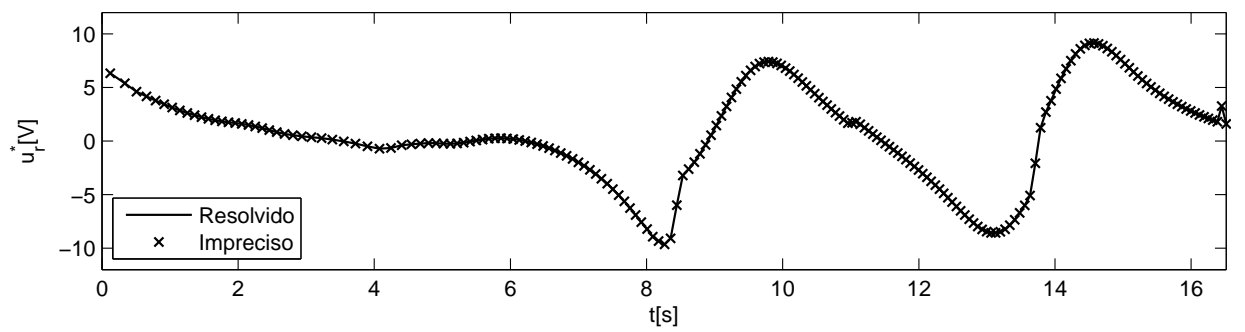

(a) Tensão ótima no motor direito $u_{l}^{*}(t)$

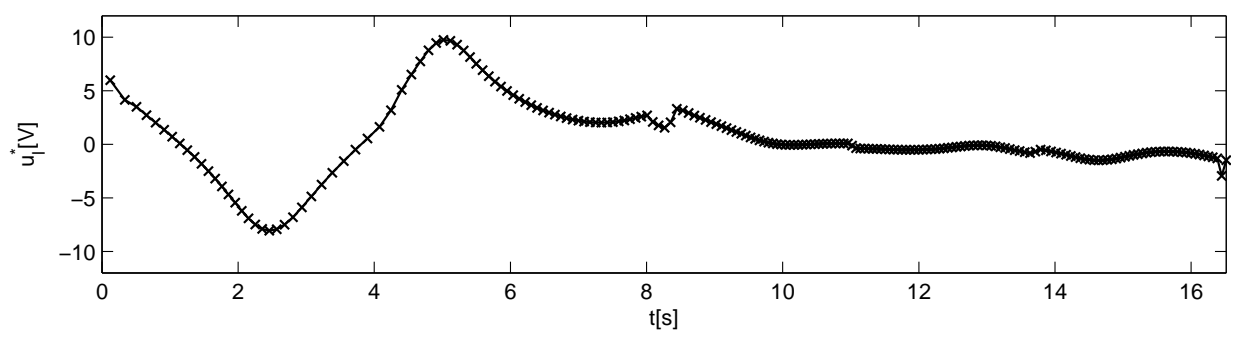

(b) Tensão ótima no motor esquerdo $u_{l}^{*}(t)$

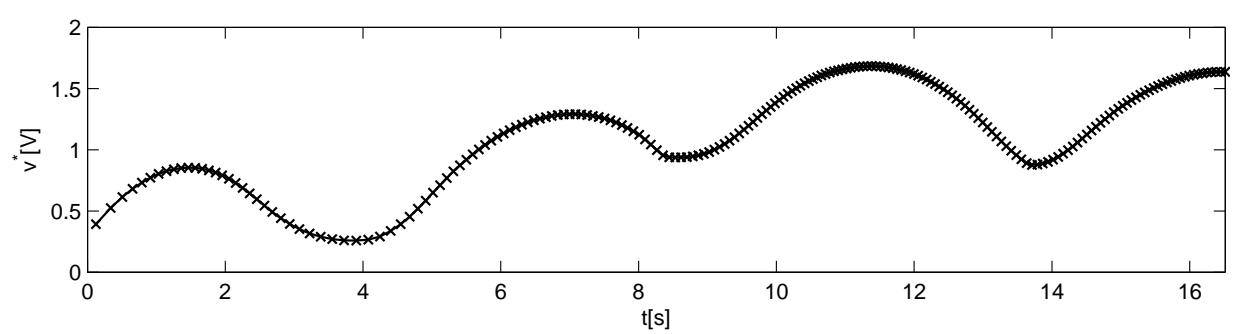

(c) Velocidade linear ótima do robô $v^{*}(t)$

Figura 25 - Gráficos de resposta para $N=200$, considerando o status final da otimização. A linha espessa representa os resultados para otimizações com status Resolvido, enquanto a com linhas cruzadas o status Impreciso.

Fonte: o autor.

\subsubsection{Quanto ao Tempo Final e à Energia Total}

Os resultados da otimização fornecem, através das equações 3.77 e 3.78, o tempo final $T_{f}$ e a energia total $E_{t}$ do percurso do robô no caminho dado. Quanto maior a discretização $N$, mais próximo ao modelo contínuo o modelo discreto é; portanto, mais os resultados da otimização se aproximam dos valores referentes ao modelo contínuo. 
Na Figura 26 pode-se observar que tanto os valores do tempo final $T_{f}$ quanto os da energia total $E_{t}$ convergem com o incremento da discretização $N$, considerando apenas os resultados da otimização que estão dentro da tolerância padrão (i.e., otimização resolvida).

Considerando a sua eficiência na otimização, sugere-se utilizar o algoritmo Mosek para a otimização de planejamento de trajetória presente neste trabalho; e considerando que a partir de $N=500$ os valores de $T_{f}$ e $E_{t}$ convergem com um erro menor do que $0,5 \%$, ao passo que o incremento da discretização aumenta o tempo de processamento na razão exponencial de 1, $18 \mathrm{dec} / \mathrm{dec}^{2}$, sugere-se utilizar $N=500$ para as otimizações.

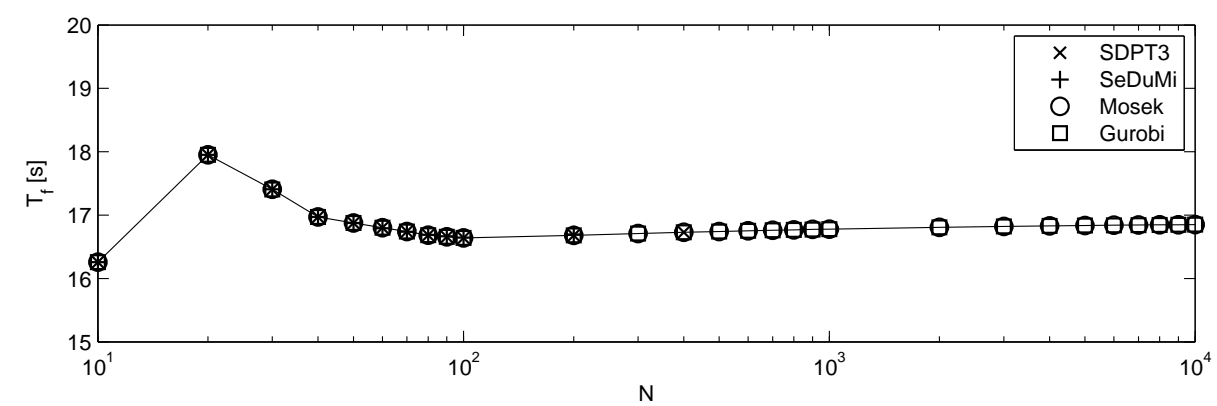

(a) Tempo final $T_{f}$

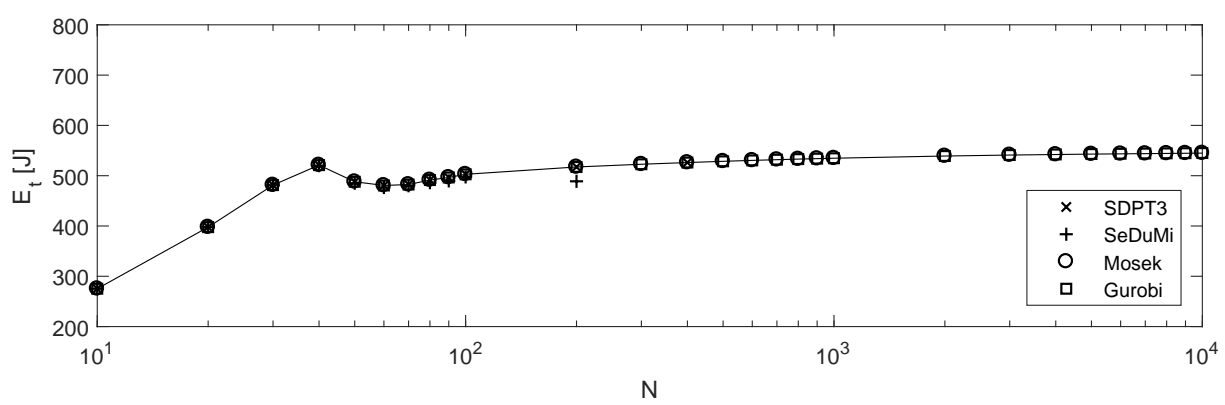

(b) Energia total $E_{t}$

Figura 26 - Resultado do Tempo final e da Energia total para os diferentes algoritmos de otimização.

Fonte: o autor.

\subsubsection{Quanto à variação do coeficiente de ponderação}

Uma outra série de otimizações foi realizada utilizando apenas o algoritmo Mosek. Com uma discretização de $N=500$, foram calculados os sinais ótimos ( $\mathbf{u}^{*}$ e $v^{*}$ ), o tempo final $\left(T_{f}\right)$ e a energia total $\left(E_{t}\right)$ para o modelo do robô percorrer o caminho $\Gamma$, considerando restrições na tensão dos motores $\mathbf{u}_{\max }=-\mathbf{u}_{\min }=\left[\begin{array}{ll}12 \mathrm{~V} & 12 \mathrm{~V}\end{array}\right]^{T}$, na velocidade $\dot{\mathbf{q}}_{\max }=$ $-\dot{\mathbf{q}}_{\min }=\left[\begin{array}{ll}2,5 \mathrm{~m} / \mathrm{s} & 1 \mathrm{rad} / \mathrm{s}\end{array}\right]^{T}$ e na aceleração $\ddot{\mathbf{q}}_{\text {max }}=-\ddot{\mathbf{q}}_{\text {min }}=\left[\begin{array}{ll}2 \mathrm{~m} / \mathrm{s}^{2} & 0,5 \mathrm{rad} / \mathrm{s}^{2}\end{array}\right]^{T}$.

A variação na série se deu no valor do coeficiente de ponderação entre tempo e energia presente na função custo $\tilde{\mathcal{J}}$, variando entre $\mu=10^{-4}$ e $\mu=10^{4}$, com passo de $\delta \mu=10^{2.5}$. 
Com base nos sinais ótimos resolvidos pela série, foram produzidos os gráficos da Figura 27. Os gráficos representam os sinais de tensão ótima dos motores direito $\left(u_{r}^{*}\right)$ e esquerdo $\left(u_{l}^{*}\right)$ e de velocidade linear ótima $\left(v^{*}\right)$ do robô em função da dariável discreta $i$. Cada linha representa o sinal para um valor distinto do coeficiente $\mu$. As setas indicam a direção da tendência do crescimento dos sinais com o aumento deste coeficiente. Para melhor entendimento do percurso, a Figura 28 ilustra os pontos no caminho $\Gamma$ relativo a cada $i$ múltiplo de 50 .

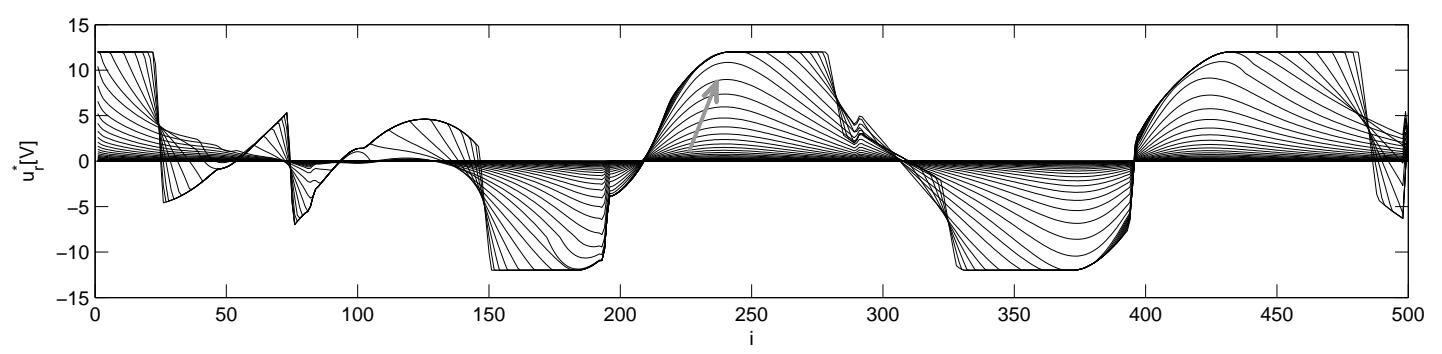

(a) Série de tensões ótimas $u_{r}^{*}$

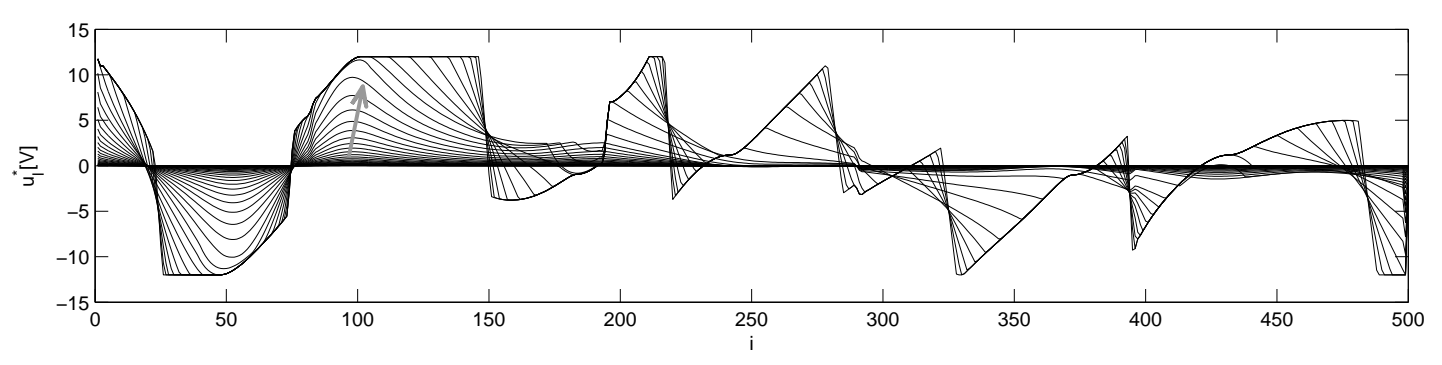

(b) Série de tensões ótimas $u_{l}^{*}$

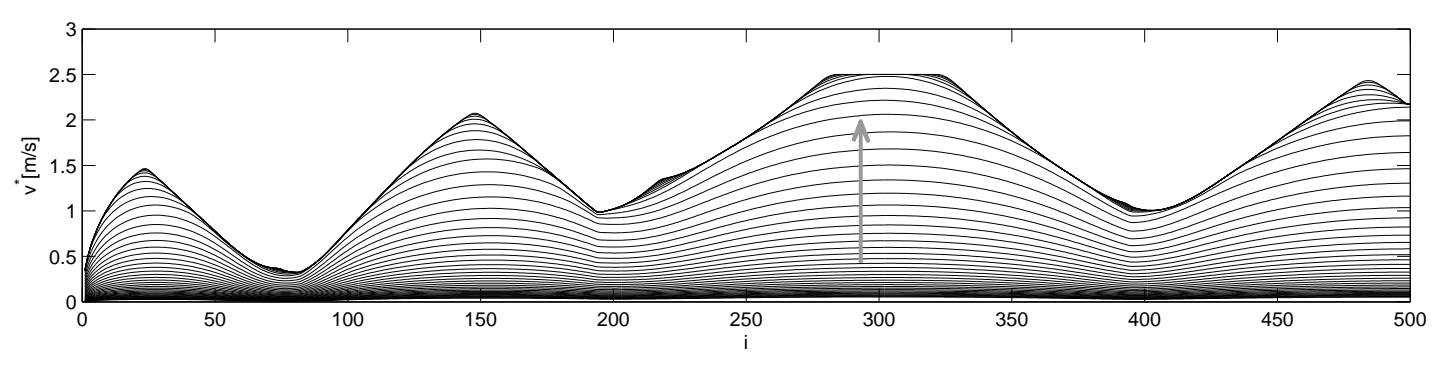

(c) Série de velocidades ótimas $v^{*}$

Figura 27 - Resposta da otimização para diferentes valores de $\mu$. As linhas representam as variáveis de otimização para valores do coeficiente de ponderação $\mu$ entre $10^{-4}$ e $10^{4}$ com passo de $\delta \mu=10^{2.5}$.

Fonte: o autor.

Para um valor de $\mu$ baixo, o planejamento prioriza o consumo energético do robô. Então, o esforço dos motores é reduzido e sua velocidade é baixa, tendo como consequência um tempo final elevado.

Com o aumento do coeficiente $\mu$, a ponderação do tempo final aumenta, levando o planejamento a aumentar as tensões dos motores e, como consequência, a velocidade linear do robô no trajeto. 
Para um valor de $\mu$ elevado, os limites impostos pelas restrições são alcançados, limitando os sinais de entrada. Podem-se observar estas saturações em alguns momentos específicos do trajeto. Por exemplo, nos patamares de tensão $u_{r}^{*}$ nas proximidades de $i=20, i=170, i=250, i=350$ e $i=450$; nos patamares $u_{l}^{*}$ nas proximidades de $i=40$ e $i=170$, e na saturação de velocidade $v^{*}$ nas proximidades de $i=300$.

Destaca-se que, observando o perfil de velocidade da Figura 27c e o caminho ilustrado na Figura 28, o planejamento antecipa as presença de curvas durante o percurso. As tensões aceleram o robô em segmentos do caminho com pouca curvatura e desaceleram antecipadamente a segmentos com curvatura mais acentuada.

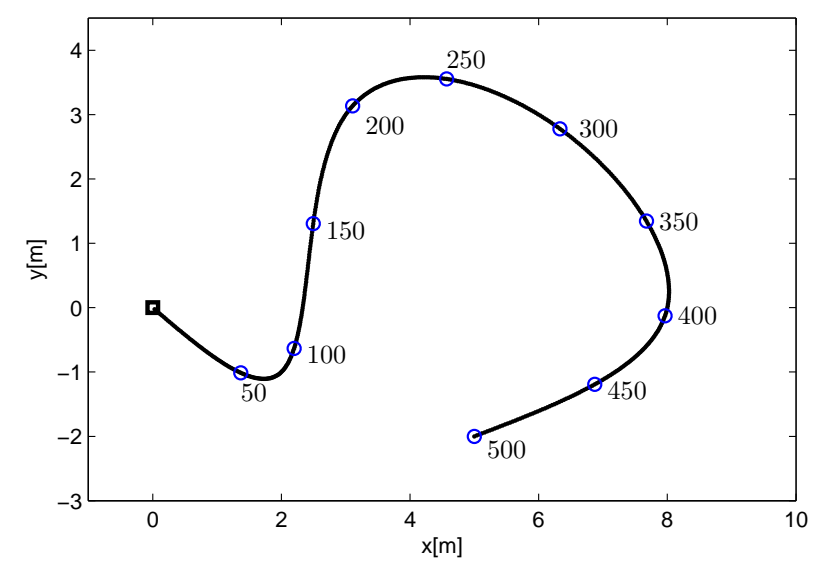

Figura 28 - Caminho $\Gamma$ discretizado com $N=500$. Os círculos representam pontos com valores de $i$ múltiplos de 50 .

Fonte: o autor.

\subsubsection{Fronteira de Pareto}

De uma forma geral, existem infinitas trajetórias de velocidade e de tensão que o robô pode realizar para percorrer o caminho solicitado. A cada uma delas estão associados um tempo final e uma energia total, respectivamente; e este conjunto infinito de pontos configura uma "Região Possível" em um gráfico $T_{f}$ vs $E_{t}$.

A otimização de trajetória é multi-critério, e a variação de seu coeficiente de ponderação $\mu$ gera uma curva no plano $T_{f}$ vs $E_{t}$ que limita a Região Possível, pois seus pontos são ótimos - configurando uma "Fronteira de Pareto", como ilustrado na Figura 29.

Os pontos cruzados são as otimizações para $\mu$ em potências inteiras de 10. Quanto menor o coeficiente $\mu$, mais ponderada a otimização é no sentido de priorizar o consumo energético. No limite, ou seja, $\mu=0$, a fronteira de Pareto converge para uma assíntota com a mínima energia total que possa ser consumida pelo robô no trajeto. Por outro lado, a otimizaçao prioriza o trajeto do robô no menor tempo possível se o coeficiente $\mu$ aumenta. 


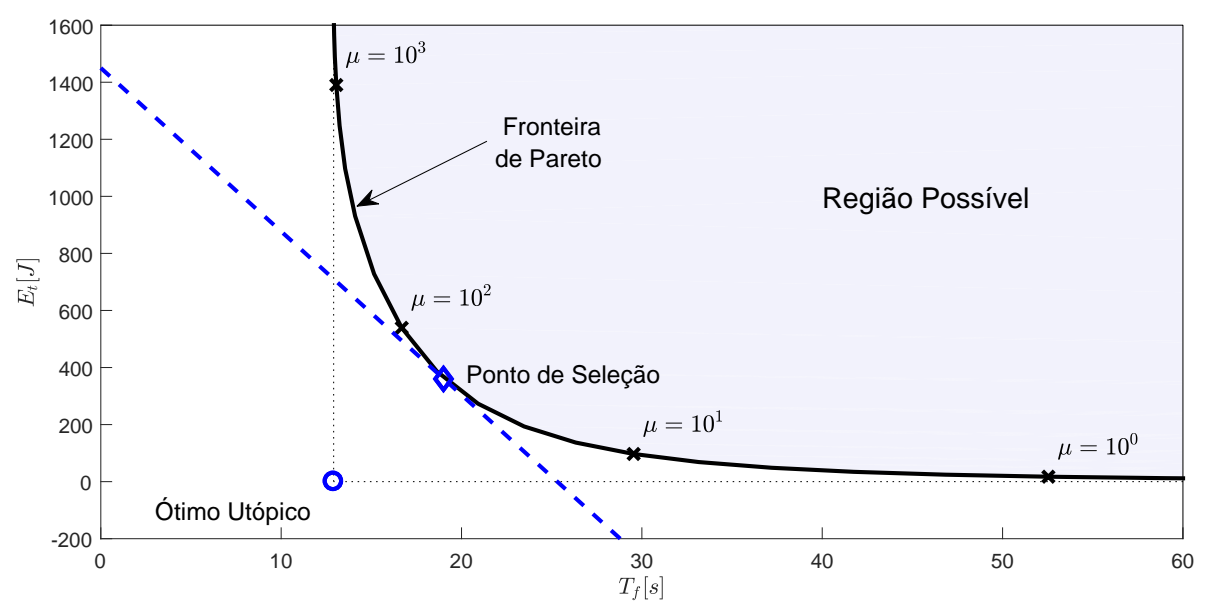

Figura 29 - Gráfico de Pareto e suas regiões com valores contínuos de $\mu$. Fonte: o autor.

No limite, ou seja, $\mu \rightarrow \infty$, a curva de Pareto converge para uma assíntota que representa este tempo mínimo.

O encontro entre estas duas assíntotas se dá em um ponto ("Ótimo Utópico", na Figura 29 representado por um círculo) onde robô atravessaria o caminho definido com o menor tempo possível e consumindo a menor energia possível $\left(T_{f \min }=12,9 \mathrm{~s}\right.$, $\left.E_{\text {tmin }}=1,4 \mathrm{~mJ}\right)$. Como estes dois critérios não podem ser atingidos ao mesmo tempo, esta condição é impossível.

O "Ponto de Seleção", representado na Figura 29 por um losango, é o ponto ótimo $\left(T_{f}^{*}, E_{t}^{*}\right)$ associado a um coeficiente de ponderação $\mu^{*}$ tal que a variação de um critério de otimização penaliza ao mínimo a variação do outro critério. Em outras palavras, a variação entre os dois critérios é mínima em ambos os sentidos. Graficamente, em um sistema de coordenadas genérico $\{x, y\} \in \mathbb{R}$, isto ocorre quando $d x=-d y$.

Como os critérios de otimização de trajetória possuem grandezas diferentes (o tempo final $T_{f}$ em segundos e a energia total $E_{t}$ em volts ao quadrado), um parâmetro de normalização se faz necessário, dado por

$$
\Lambda=\frac{\Delta E_{t}}{\Delta T_{f}}
$$

que representa uma relação de "custo" da energia total em relação ao tempo de percurso, dado em $J / s$. O ponto de inflexão $\left(T_{f}^{*}, E_{t}^{*}\right)$ será então aquele no qual

$$
\frac{d E_{t}\left(T_{f}\right)}{d T_{f}}\left(T_{f}^{*}\right)=-\Lambda
$$

e a tangente da curva em um gráfico normalizado por $\Lambda$ tem inclinação de $-45^{\circ}$. 


\subsection{SINTONIA DO COEFICIENTE DE PONDERAÇÃO}

Observa-se graficamente, através dos valores numéricos das otimizações, que a curva da Fronteira de Pareto possui um comportamento hiperbólico, como ilustra a Figura 30a.

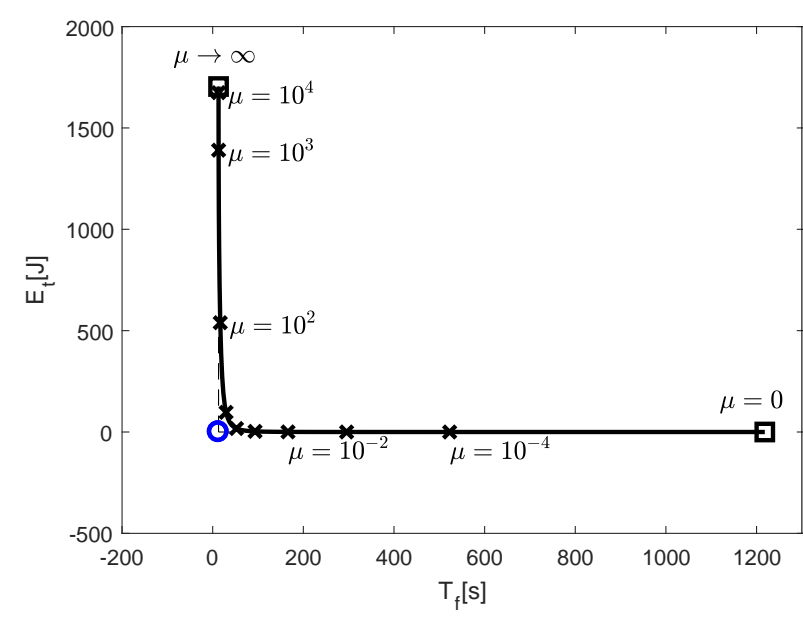

(a) Pareto em escala linear

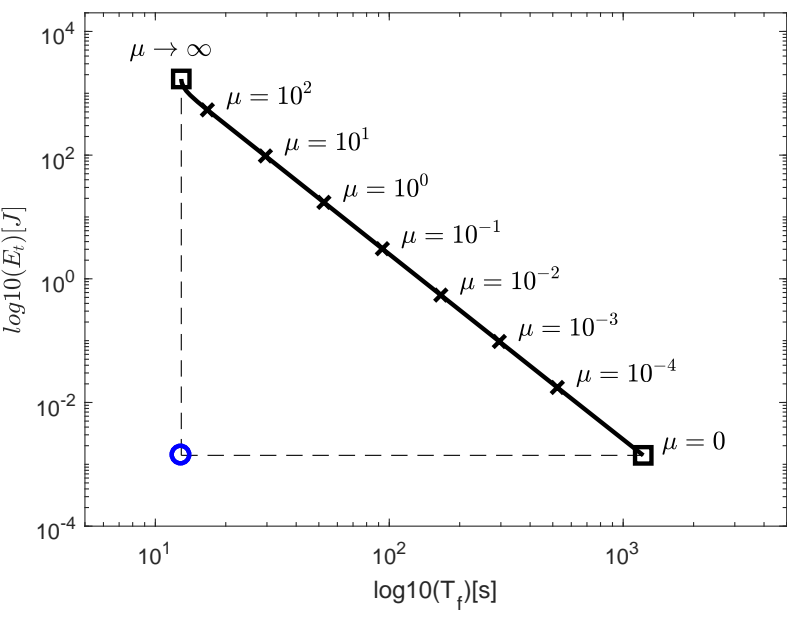

(b) Pareto em escala logarítmica

Figura 30 - Gráficos de Pareto em escala linear e logarítmica. O círculo representa o ponto Ótimo Utópico, e os quadrados representam os limites mínimos e máximos de otimização.

Fonte: o autor

Este comportamento se confirma quando se traça o Pareto com seus critérios em uma escala logarítmica, como mostra a Subfigura 30b. As fronteiras se revelam aparentemente como segmentos de reta entre os pontos de saturação, representados nas figuras por quadrados. Nos gráficos, percebe-se um comportamento linear em boa parte do espectro de variação do coeficiente $\mu$, excetuando na região de saturação.

Com a limitação da tensão dos motores $u$, por mais que se aumente o valor do coeficiente de ponderação $\mu \rightarrow \infty$ priorizando um tempo mínimo, o robô não consegue realizar o percurso em menos tempo que um determinado limite (neste caso específico de $\left.T_{f \min }=12,9 \mathrm{~s}\right)$. Assim, a energia total também se limita em $E_{t \max }=1,743 \mathrm{~J}$.

Por outro lado, mesmo se $\mu=0$, o robô precisa percorrer o caminho, que possui curvaturas e, portanto, ao menos um mínimo valor de diferencial de tensão deve ocorrer. A energia mínima para tal tarefa é de $E_{t \min }=1,4 m V^{2}$, em um tempo total de $T_{f \max }=1218 \mathrm{~s}$.

\subsubsection{Interpolação da Curva da Fronteira de Pareto}

Supõe-se que a relação entre o tempo final $T_{f}$ e a energia total $E_{t}$ do robô no percurso seja uma função $E_{t}=f\left(T_{f}\right): \mathbb{R} \rightarrow \mathbb{R}$, regida pela equação

$$
E_{t}\left(T_{f}\right)=\beta \cdot T_{f}^{\alpha},
$$


onde $\alpha, \beta \in \mathbb{R}$ são coeficientes escalares constantes e independentes de $E_{t}$ e $T_{f}$. Aplicandose o logaritmo na Equação 3.82, simbolizando-o da forma $(\hat{\cdot})=\log 10(\cdot)$, tem-se a equação

$$
\hat{E}_{t}\left(\hat{T}_{f}\right)=\hat{\beta}+\alpha \cdot \hat{T}_{f}
$$

que é uma função afim de $\hat{T}_{f}$ para $\hat{E}_{t}$, onde $\alpha$ é o coeficiente angular e $\hat{\beta}$ é o coeficiente linear da reta traçada por esta função no plano loglog.

Então, dados dois pares de resultados da otimização $\left(T_{f 1}, E_{t 1}\right)$ e $\left(T_{f 2}, E_{t 2}\right)$ na reta, os coeficientes $\alpha$ e $\beta$ podem ser calculados pelas equações

$$
\begin{aligned}
& \alpha=\frac{\hat{E}_{t 2}-\hat{E}_{t 1}}{\hat{T}_{f 2}-\hat{T}_{f 1}}, \\
& \beta=10^{\left(\hat{E}_{t 1}-\alpha \hat{T}_{f 1}\right)}=10^{\left(\hat{E}_{t 2}-\alpha \hat{T}_{f 2}\right)} .
\end{aligned}
$$

Derivando a Equação 3.82, tem-se

$$
\frac{d E_{t}\left(T_{f}\right)}{d T_{f}}=\alpha \beta \cdot T_{f}^{(\alpha-1)}
$$

que no ponto de seleção $\left(T_{f}^{*}, E_{t}^{*}\right)$ tem a inclinação dada pelo coeficiente de normalização (Eq, 3.81), ou seja,

$$
\alpha \beta \cdot T_{f}^{*(\alpha-1)}=-\Lambda
$$

Portanto, as coordenadas do ponto de seleção podem ser encontradas pelas equações

$$
\begin{aligned}
& T_{f}^{*}=\left(-\frac{\Lambda}{\alpha \beta}\right)^{\frac{1}{(\alpha-1)}}, \\
& E_{t}^{*}=\beta \cdot\left(T_{f}^{*}\right)^{\alpha},
\end{aligned}
$$

onde $\Lambda$ é um parâmetro selecionado pelo engenheiro de controle, e os coeficientes $\alpha$ e $\beta$ são calculados pelas equações 3.84 e 3.85 .

\subsubsection{Obtenção dos coeficientes $\alpha$ e $\beta$}

Seja então a otimização de trajetória com o coeficiente de ponderação $\mu=10^{-4}$. Considere-se que esta otimização forneça um primeiro par de dados $\left(T_{f 1}, E_{t 1}\right)$ tal que $T_{f 1}=522,85 s$ e $E_{t 1}=0,0175 V^{2}$. Então, para cada $\mu^{i}, i=-3, \ldots, 4$, considera-se que a otimização forneça o segundo par de dados $\left(T_{f 2}, E_{t 2}\right)$. Através das equações 3.84 e 3.85, calculam-se os valores dos coeficientes $\alpha$ e $\beta$. Os resultados destes cálculos são apresentados na Tabela 2. 
Tabela 2 - Coeficientes $\alpha$ e $\beta$ calculados para alguns valores de $\mu$

\begin{tabular}{lrrcc}
\hline$\mu$ & $T_{f 2}[s]$ & $E_{t 2}[\mathrm{~J}]$ & $\alpha$ & $\beta$ \\
\hline $10^{-3}$ & 295,46 & 0,0969 & $-2,9998$ & $2,4979 \cdot 10^{6}$ \\
$10^{-2}$ & 166,11 & 0,5456 & $-2,9999$ & $2,4996 \cdot 10^{6}$ \\
$10^{-1}$ & 93,428 & 3,0662 & $-2,9999$ & $2,4999 \cdot 10^{6}$ \\
$10^{0}$ & 52,541 & 17,241 & $-3,0000$ & $2,5001 \cdot 10^{6}$ \\
$10^{1}$ & 29,546 & 96,949 & $-3,0000$ & $2,5001 \cdot 10^{6}$ \\
$10^{2}$ & 16,685 & 539,17 & $-3,0004$ & $2,5077 \cdot 10^{6}$ \\
$10^{3}$ & 13,057 & 1390,3 & $-3,0578$ & $3,5901 \cdot 10^{6}$ \\
$10^{4}$ & 12,910 & 1674,0 & $-3,0986$ & $4,6356 \cdot 10^{6}$ \\
\hline \multicolumn{5}{c}{ Fonte: o autor }
\end{tabular}

Observa-se pelos dados apresentados que existe uma certa linearidade nos coeficientes $\alpha$ e $\beta$ quando calculados com base nos resultados da otimização com $\mu$ variando entre $\mu=10^{-3}$ e $\mu=10^{1}$. A Figura 31 ilustra a curva de Pareto com os dados experimentais de otimização e as curvas da forma $E_{t}=\beta \cdot\left(T_{f}\right)^{\alpha}$ para cada conjunto de coeficientes $\alpha$ e $\beta$ calculados e apresentados na Tabela 2 .

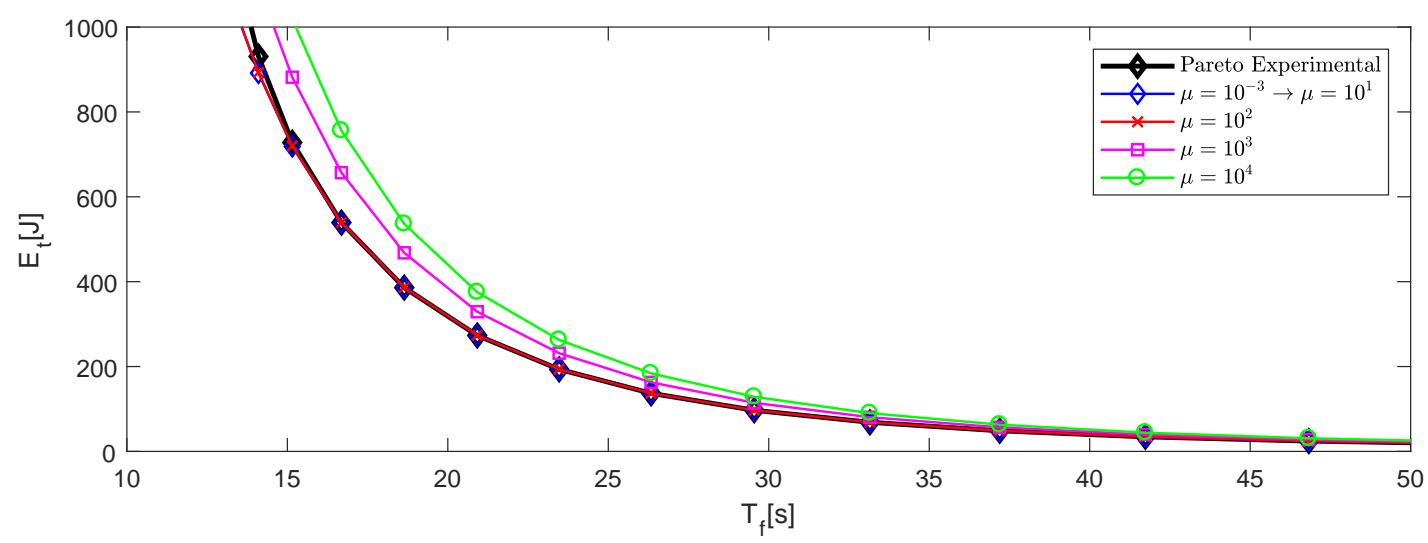

Figura 31 - Gráficos das equações exponenciais entre $T_{f}$ e $E_{t}$ para diferentes valores de $\mu$. As curvas para valores entre $\mu=10^{-3}$ e $\mu=10^{1}$ foram traçadas em uma única linha azul com pontos em losango.

Fonte: o autor.

Considera-se coerente que os valores experimentais dos coeficientes $\alpha$ e $\beta$ sejam oferecidos pelos dados de tempo final e energia total resultantes das otimizações realizadas com $\mu=-4$ e $\mu=0$, Então, $\alpha=3$ e $\beta=2,5 \cdot 10^{6}$.

\subsubsection{Relação entre $\mu$ e $T_{f}$}

Observa-se também, com os dados resultantes das mesmas otimizações experimentais, que a relação entre o coeficiente $\mu$ e o tempo final $T_{f}$ é exponencial, como mostra a Figura 32a. Este comportamento fica mais claro quando se traça um gráfico loglog destes dados, como ilustra a Figura 32b. 


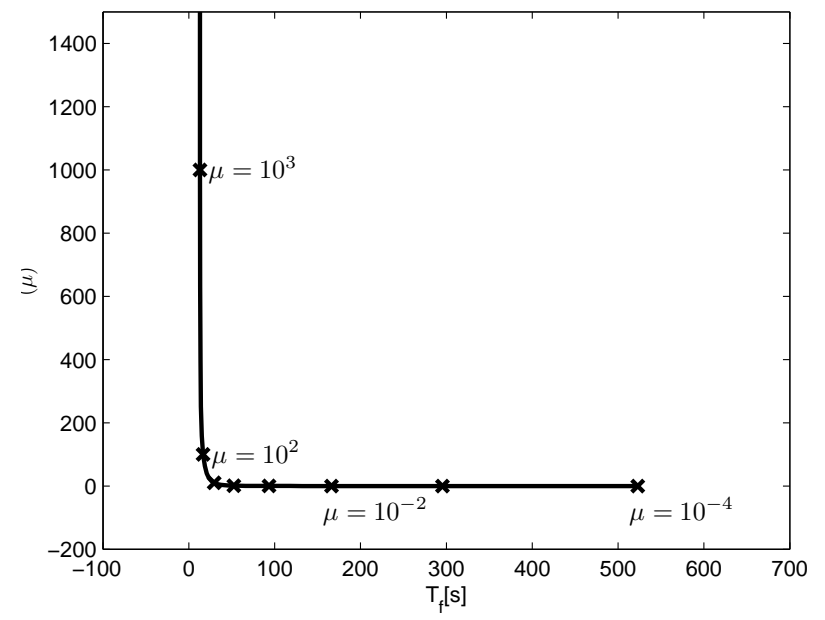

(a) Gráfico em escala linear

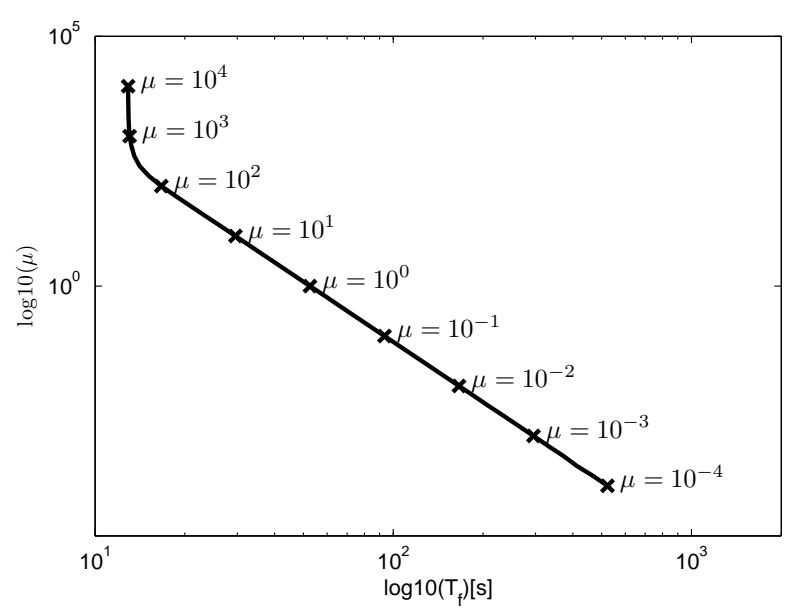

(b) Gráfico em escala logarítmica

Figura 32 - Gráficos $T_{f}$ vs $\mu$ em escala linear e logarítmica,

Fonte: o autor.

Parte-se do pressuposto que a relação entre o tempo final $T_{f}$ e o coeficiente de ponderação $\mu$ seja uma função $\mu=f\left(T_{f}\right): \mathbb{R} \rightarrow \mathbb{R}$, dada pela equação

$$
\mu\left(T_{f}\right)=\kappa \cdot T_{f}^{\nu}
$$

onde $\nu, \kappa \in \mathbb{R}$ são coeficientes escalares constantes e independentes de $\mu$ e $T_{f}$. Aplicando-se o logaritmo na Equação 3.90, tem-se

$$
\hat{\mu}\left(\hat{T}_{f}\right)=\hat{\kappa}+\nu \cdot \hat{T}_{f}
$$

que é uma função afim de $\hat{T}_{f}$ para $\hat{\mu}$, onde $\nu$ é o coeficiente angular e $\hat{\kappa}$ é o coeficiente linear da reta traçada por esta função no plano loglog.

Com dois pares de pontos $\left(T_{f 1}, \mu_{1}\right)$ e $\left(T_{f 2}, \mu_{2}\right)$, os coeficientes $\nu$ e $\kappa$ podem ser obtidos pelas equações

$$
\begin{aligned}
& \nu=\frac{\hat{\mu}_{2}-\hat{\mu}_{1}}{\hat{T}_{f 2}-\hat{T}_{f 1}}, \\
& \kappa=10^{\left(\hat{\mu}_{1}-\nu \hat{T}_{f 1}\right)}=10^{\left(\hat{\mu}_{2}-\nu \hat{T}_{f 2}\right)} .
\end{aligned}
$$

\subsubsection{Cálculo experimental dos coeficientes $\nu$ e $\kappa$}

Para um coeficiente de ponderação $\mu=10^{-4}$, o tempo final calculado pela otimização de trajetória é de $T_{f 1}=522,85 \mathrm{~s}$; seja este o primeiro par de dados da equação 3.92. Então, sejam as otimizações com $\mu=10^{i}, i=-3, \ldots, 4$. Os resultados de tempo final dessas otimizações, juntamente com os coeficientes de ponderação, fornecem o segundo par de dados $\left(T_{f 2}, \mu_{2}\right)$. Através das equações 3.92 e 3.93, calculam-se os valores dos coeficientes $\nu$ e $\kappa$, que são apresentados na Tabela 3 . 
Tabela 3 - Coeficientes $\nu$ e $\kappa$ calculados para alguns valores de $\mu$.

\begin{tabular}{lrcc}
\hline$\mu$ & $T_{f 2}[s]$ & $\nu$ & $\kappa$ \\
\hline $10^{-3}$ & 295,46 & $-4,0343$ & $9,2651 \cdot 10^{6}$ \\
$10^{-2}$ & 166,11 & $-4,0162$ & $8,2725 \cdot 10^{6}$ \\
$10^{-1}$ & 93,428 & $-4,0112$ & $8,0173 \cdot 10^{6}$ \\
$10^{0}$ & 52,541 & $-4,0085$ & $7,8806 \cdot 10^{6}$ \\
$10^{1}$ & 29,546 & $-4,0068$ & $7,7972 \cdot 10^{6}$ \\
$10^{2}$ & 16,685 & $-4,0106$ & $7,9849 \cdot 10^{6}$ \\
$10^{3}$ & 13,057 & $-4,3681$ & $7,4839 \cdot 10^{6}$ \\
$10^{4}$ & 12,910 & $-4,9768$ & $3,3804 \cdot 10^{6}$ \\
\hline \multicolumn{4}{c}{ Fonte: o autor }
\end{tabular}

Observa-se pelos dados apresentados que existe uma ligeira linearidade nos coeficientes $\nu$ e $\kappa$ quando calculados com base nos resultados da otimização, com $\mu$ variando entre $\mu=10^{-1}$ e $\mu=10^{2}$.

A Figura 33 ilustra a curva $T_{f}$ vs $\mu$ com os dados experimentais de otimização e uma família de curvas da forma $\mu=\kappa \cdot\left(T_{f}\right)^{\nu}$ para cada conjunto de coeficientes $\nu$ e $\kappa$ calculados e apresentados na Tabela 3. Percebe-se que as curvas geradas com $\mu$ na faixa entre $\mu=10^{-1}$ e $\mu=10^{2}$ nesta escala possuem uma certa concordância, motivo pela qual são traçadas em uma linha apenas.

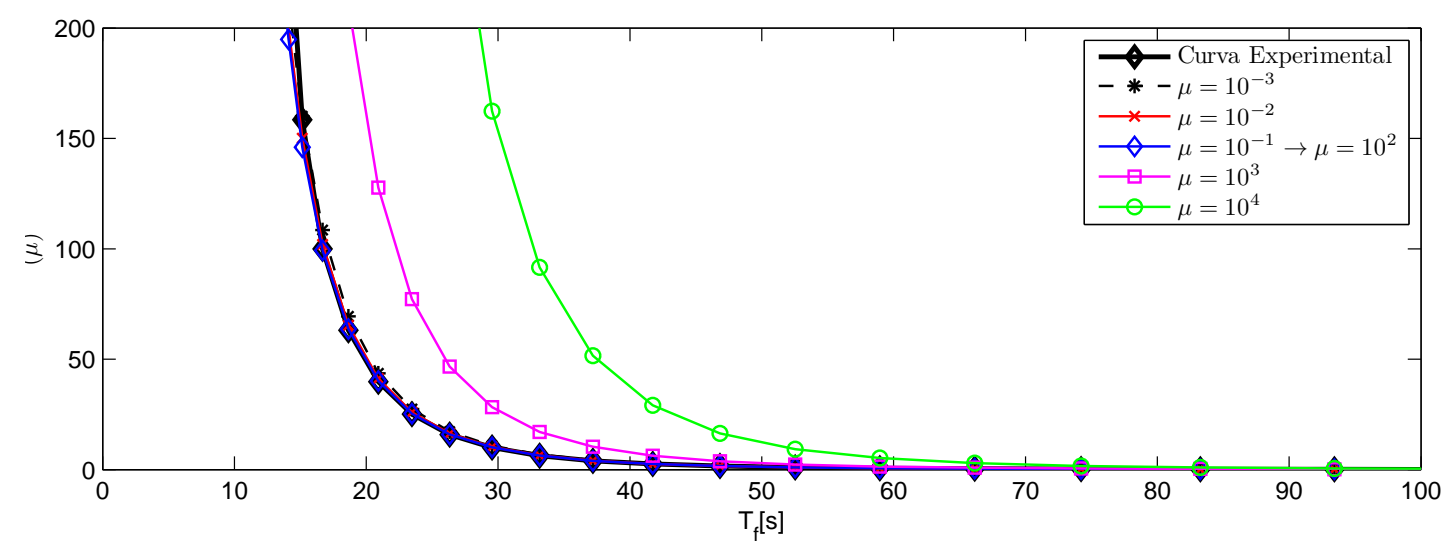

Figura 33 - Gráficos das equações exponenciais entre $T_{f}$ e $\mu$ para diferentes valores de $\mu$. As curvas para valores entre $\mu=10^{-1}$ e $\mu=10^{2}$ foram traçadas em uma única linha azul com pontos em losango.

Fonte: o autor.

Apesar dos coeficientes não apresentarem uma linearidade tão expressiva em termos numéricos, pela relativa concordância das curvas, considera-se coerente e oportuno que os dados para os cálculos dos coeficientes $\mu$ e $\kappa$ sejam oferecidos pelos dados resultantes das otimizações realizadas com $\mu=-4$ e $\mu=0$. Como resultado, $\mu=-4,0085$ e $\kappa=7,8806 \cdot 10^{6}$. 


\subsubsection{Algoritmo de sintonia automática do coeficiente de ponderação}

Seja o robô apresentado na seção 3.5. Seja também um caminho $\Gamma$ definido pelos $N+1$ pontos discretos $\mathbf{p}_{i}=\left[\begin{array}{ll}x_{i} & y_{i}\end{array}\right]^{T}, i=0, \ldots, N$ no plano $\left\{X_{O}, Y_{O}\right\}$. O objetivo do algoritmo é encontrar um coeficiente de ponderação $\mu$ tal que os objetivos de tempo final $T_{f}$ e de energia total $E_{t}$ da otimização, normalizados por um coeficiente $\Lambda$, configurem um ponto de seleção na fronteira de Pareto, ou seja, não possam ser minimizados sem aumentar significativamente o seu objetivo complementar. O procedimento em pseudocódigo é apresentado no Algoritmo 4.

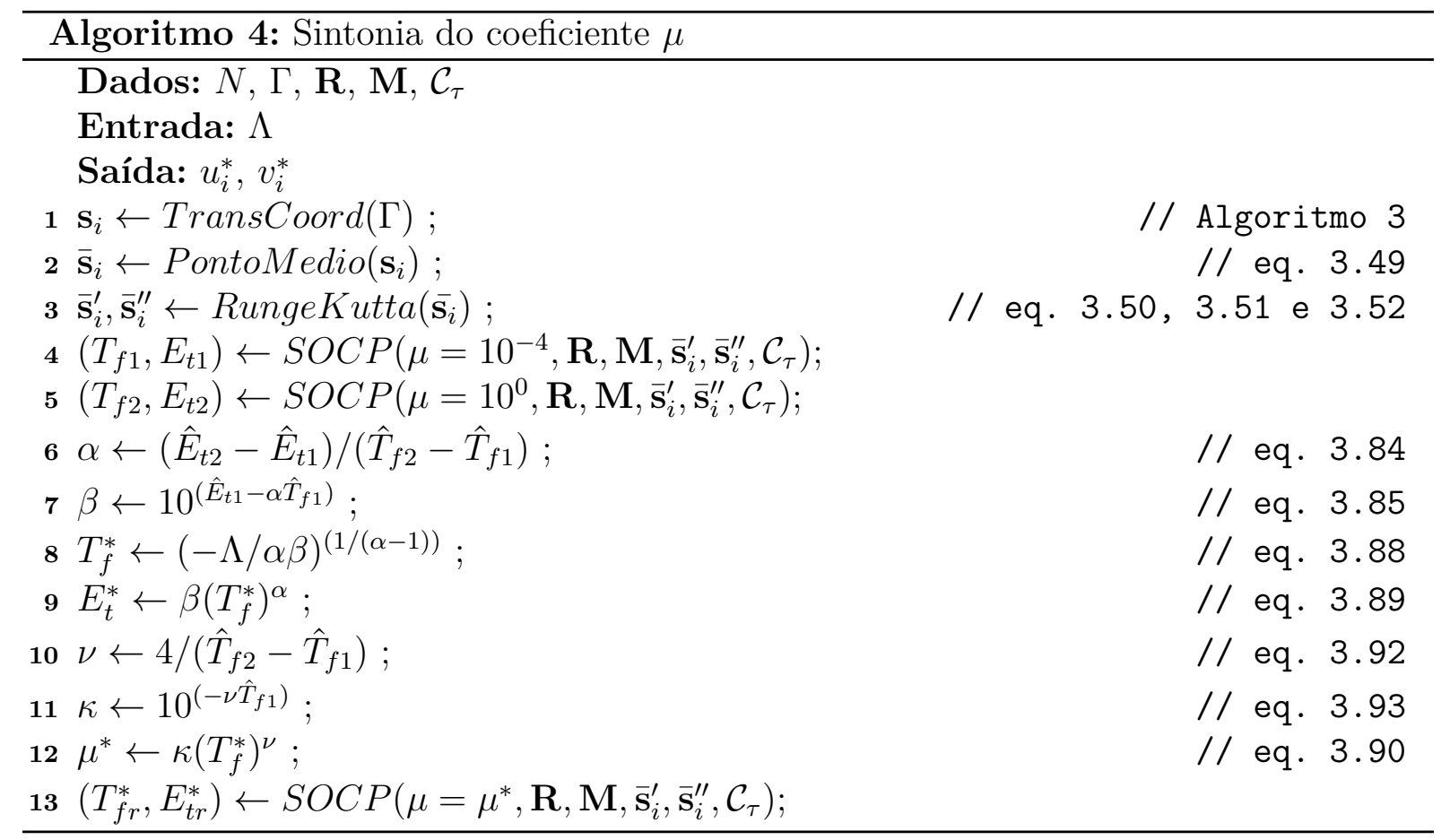

Inicialmente, o Algoritmo 3 é executado para transformar os pontos $\mathbf{p}_{i}=\left[\begin{array}{ll}x_{i} & y_{i}\end{array}\right]^{T}$, $i=0, \ldots, N$ na configuração alternativa $\mathbf{s}_{i}=\left[\begin{array}{ll}\gamma_{i} & \theta_{i}\end{array}\right]^{T}, i=0, \ldots, N$. Então, são estimados os pontos médios $\overline{\mathbf{s}}_{i}$ (eq. 3.49), suas derivadas de primeira ordem $\overline{\mathbf{s}}_{i}^{\prime}$ (eq. 3.50) e suas derivadas de segunda ordem $\overline{\mathbf{s}}_{i}^{\prime \prime}$ (eq. 3.51 e 3.52).

Uma primeira execução da SOCP é realizada com o coeficiente de ponderação setado em $\mu=10^{-4}$, gerando o primeiro conjunto de dados $\left(T_{f 1}, E_{t 1}\right)$. Então, outra execução da programação é realizada para gerar um segundo conjunto de dados $\left(T_{f 2}, E_{t 2}\right)$, desta vez $\operatorname{com} \mu=1$.

Com os dados gerados pelas execuções da SOCP, é possível calcular os coeficientes $\alpha$ e $\beta$ (eq, 3.84 e 3.85) que são necessários para se encontrar as coordenadas do ponto de seleção $\left(T_{f}^{*}, E_{t}^{*}\right)$ pelas equações 3.88 e 3.89 . Em seguida calculam-se os coeficientes $\nu$ e $\kappa$ (eq. 3.92 e 3.93 ) para se encontrar o coeficiente de ponderação $\mu^{*}$ relativo ao ponto de seleção. 
Para validar o procedimento proposto, o Algoritmo 4 foi executado para uma série de valores do coeficiente de normalização $\Lambda=[1,2,5,10,20,50,100,200,500]$. Para cada execução, o algoritmo oferece o valor do coeficiente de ponderação $\mu^{*}$ tal que sintonize a otimização no ponto de seleção $\left(T_{f}^{*}, E_{t}^{*}\right)$. A Figura 34 ilustra as fronteiras de Pareto com seus pontos de seleção para as nove execuções do algoritmo.

Para um coeficiente de normalização $\Lambda=1$, ou seja, para um custo relativo a $1 J$ equivalente ao custo de $1 s$ de tarefa realizada pelo robô, o ponto ótimo em que a diminuição do tempo em que o robô realiza a tarefa não irá aumentar significativamente a energia consumida para a realização da tarefa é tal que $T_{f}^{*}=52,33 s$ e $E_{t}^{*}=17$,44J. Para tanto, o coeficiente de ponderação que deve ser imposto à programação cônica de segunda ordem é de $\mu^{*}=1,016$, como ilustra o gráfico na subfigura 34a.

Conforme se aumenta a relação de custo entre a energia total e o tempo final dado pelo coeficiente $\Lambda$, a proporção gráfica entre os objetivos faz com que uma reta com inclinação $-\Lambda$ seja tangente à Fronteira de Pareto em um ponto com um tempo final $T_{f}=T_{f}^{*}$ menor e, por consequência, uma energia total $E_{t}=E_{t}^{*}$ maior. O coeficiente de ponderação $\mu=\mu^{*}$ que sintoniza o par $\left(T_{f}^{*}, E_{t}^{*}\right)$ também aumenta com o aumento da proporção de custo $\Lambda$. Observa-se este comportamento nos gráficos iustrados nas subfiguras 34 a até 34 i.

As quatro colunas da esquerda da Tabela 4 ilustram os resultados numéricos das execuções do Algoritmo: o par $\left(T_{f}^{*}, E_{t}^{*}\right)$ estimado pela consideração de que a Fronteira de Pareto é uma função logarítmica, e o coeficiente ótimo $\mu^{*}$ relativo a esta estimação. Com os coeficientes de ponderação sintonizados pelo Algoritmo, foram executadas otimizações, e seus resultados ilustrados como um par $\left(T_{f r}^{*}, E_{t r}^{*}\right)$.

Tabela 4 - Resultados do Algoritmo de sintonia do coeficiente de ponderação $\mu$ e seu erro relativo à otimização para alguns valores de $\Lambda$.

\begin{tabular}{|c|c|c|c|c|c|c|}
\hline \multirow[b]{2}{*}{$\Lambda$} & \multicolumn{3}{|c|}{ Algoritmo } & \multicolumn{2}{|c|}{ SOCP } & Erro \\
\hline & $T_{f}^{*}[s]$ & $E_{t}^{*}[\mathrm{~J}]$ & $\mu^{*}$ & $T_{f r}^{*}[s]$ & $E_{t r}^{*}[\mathrm{~J}]$ & $\varepsilon[\%]$ \\
\hline 1 & 52,3344 & 17,4450 & 1,016 & 52,3325 & 17,4470 & 0,0075 \\
\hline 2 & 44,0077 & & & & &, 0747 \\
\hline 5 & & 58,33 & 5,0 & & & 0,1705 \\
\hline 10 & 296 & & & & & 0,2455 \\
\hline 20 & 24,7 & 164,9 & & & & 0,3172 \\
\hline 50 & 19,6 & 328, & 51,22 & & 330 , & 0,4171 \\
\hline 100 & & 551,65 & 102,6 & & & 0,3436 \\
\hline 200 & 13,9163 & 927,7659 & 205,5 & & 844,0361 & 6,9550 \\
\hline 500 & 11,0672 & 1844,6000 & 514,7 & 13,3558 & 1182,0000 & 36,5966 \\
\hline
\end{tabular}


Os erros relativos foram calculados com o critério:

$$
\varepsilon=\frac{\frac{\left|T_{f r}^{*}-T_{f}^{*}\right|}{T_{f r}^{*}}+\frac{\left|E_{t r}^{*}-E_{t}^{*}\right|}{E_{t r}^{*}}}{2} \times 100
$$

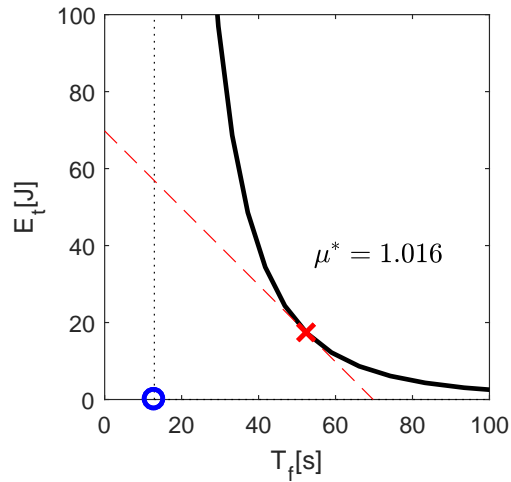

(a) $\Lambda=1$

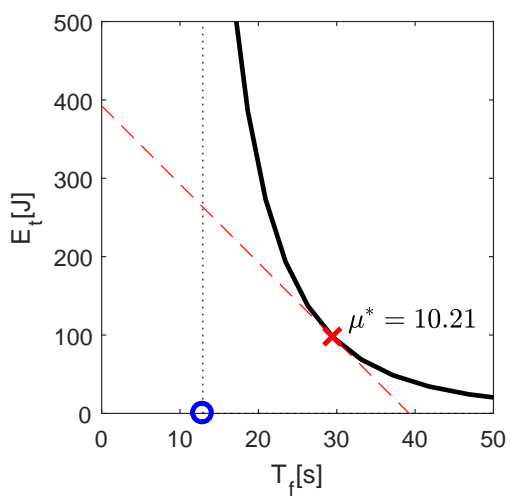

(d) $\Lambda=10$

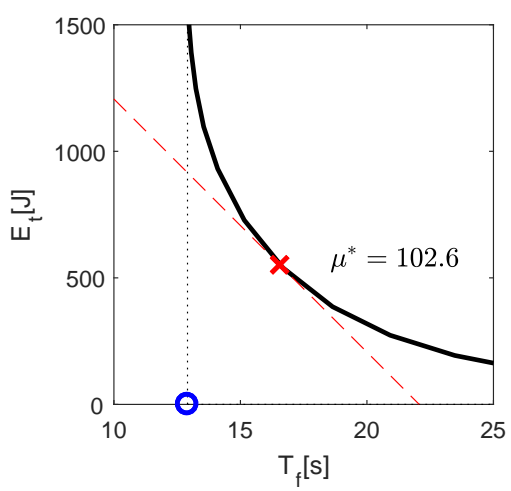

(g) $\Lambda=100$

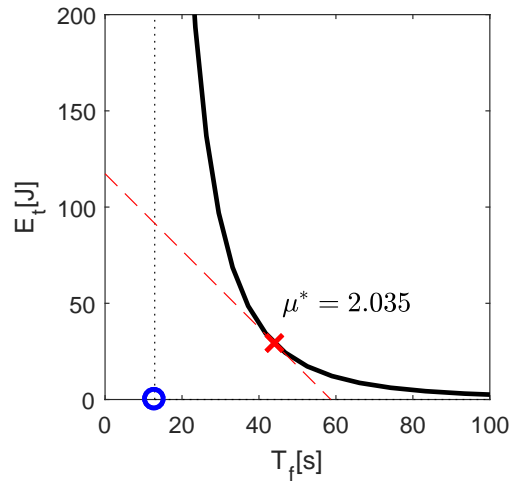

(b) $\Lambda=2$

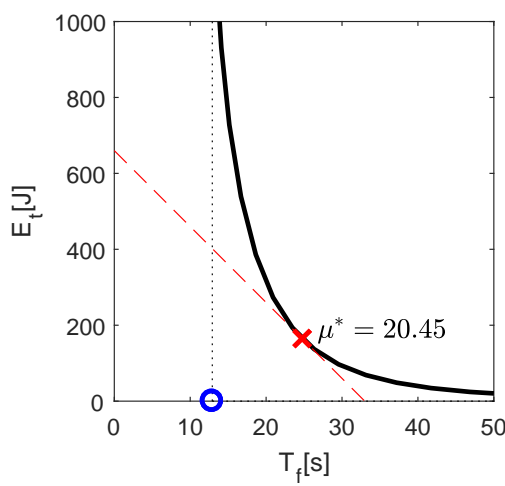

(e) $\Lambda=20$

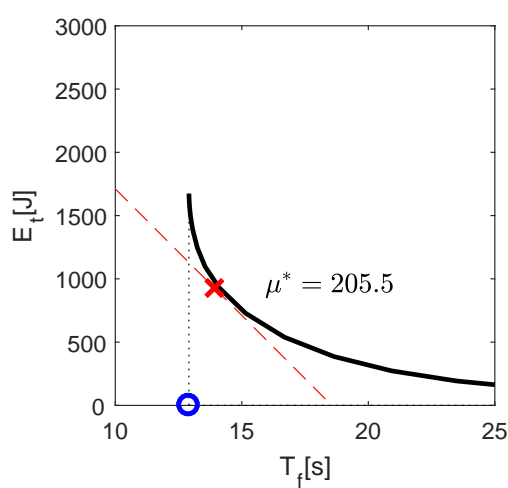

(h) $\Lambda=200$

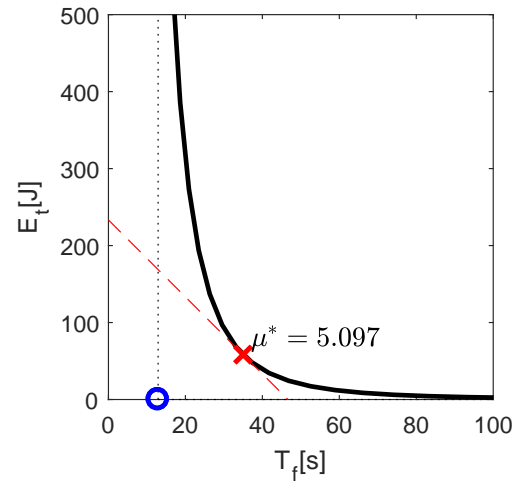

(c) $\Lambda=5$

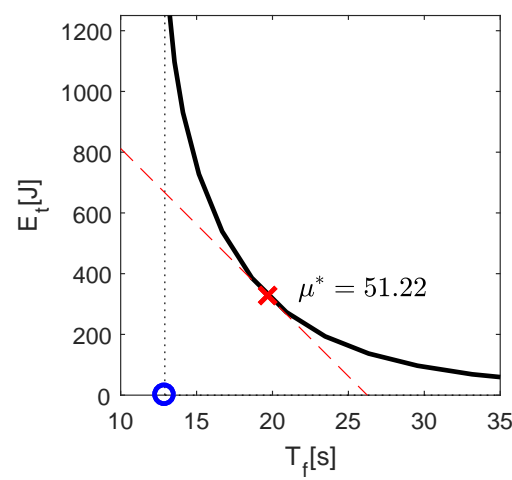

(f) $\Lambda=50$

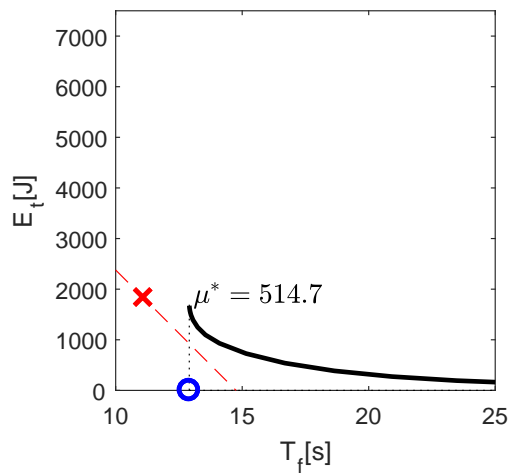

(i) $\Lambda=500$

Figura 34 - Gráficos de Pareto com algumas sintonias de $\mu$ em função de $\Lambda$. O círculo ilustra o ótimo utópico, e o ponto em cruz representa o ponto de seleção $\left(T_{f}^{*}, E_{t}^{*}\right)$ calculado pelo algoritmo de sintonia. A linha tracejada ilustra a reta tangente ao ponto de seleção, com inclinação de $-\Lambda$.

Fonte: o autor. 
Os resultados das otimizações com o coeficiente ótimo $\mu^{*}$ e seus erros relativos $\varepsilon$ para cada valor de $\Lambda$ são apresentados nas últimas colunas da Tabela 4.

Observa-se, tanto na Tabela 4 quanto nas subfiguras $34 \mathrm{~h}$ e $34 \mathrm{i}$, que para uma relação de custo $\Lambda \gg 100$ o erro relativo é $\varepsilon \gg 1 \%$, ou seja, a variação $\delta E_{t} / \delta T_{f}$ para variações de $\mu$ não pode ser alcançada pela especificação da relação de custo $\Lambda=\Delta E_{t} / \Delta T_{f}$ dada pelo usuário.

Isto ocorre devido ao fato de que, para um coeficiente de ponderação $\mu$ muito elevado (no caso do robô e da curva dada, $\mu \gg 100$ ), tanto os sinais de tensão $\mathbf{u}(t)$, de velocidade $\dot{\mathbf{q}}(t)$ e de aceleração $\ddot{\mathbf{q}}(t)$ entram em saturação, limitados pelas restrições impostas que as mantêm dentro do conjunto convexo $\mathcal{C}_{t}$. Estas limitações impedem o robô de consumir mais do que uma determinada energia para percorrer o caminho definido.

\subsection{CONCLUSÃO DO CAPÍTULO}

Os modelos cinemático e dinâmico do robô uniciclo diferencial classicamente são apresentados nas coordenadas generalizadas $\boldsymbol{\xi} \in \mathbb{R}^{3}$. Como o controle para esta topologia robótica, $\mathbf{u} \in \mathbb{R}^{2}$, tem dimensionalidade menor do que as coordenadas classicamente apresentadas, o modelo dinâmico não pode ser utilizado como restrição no problema de otimização convexa descrito inicialmente pelo Problema 3.2.

Isto ocorre porque o Problema possui quatro equações como restrições de igualdade - três relativas à dinâmica do sistema (3.33) e uma que relaciona as variáveis auxiliares

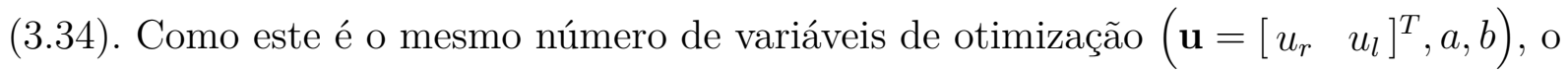
Pproblema possui uma única solução e, portanto, não pode ser otimizado.

Porém, como o robô uniciclo diferencial se move em um caminho específico, a relação entre o ponto $\mathbf{p}$ e o ângulo $\theta$ já é pré-determinado pela própria curvatura do caminho dado. Este princípio motivou a transformação de variáveis proposta pelo Lema 3.2 na qual as coordenadas generalizadas alternativas dadas por q reduzem a equação dinâmica do sistema (3.33).

Esta transformação possibilita a utilização do robô uniciclo diferencial em um problema de otimização. Então, uma adaptação do método proposto por (VERSCHEURE et al., 2008), e posteriormente adaptado por (LIPP; BOYD, 2014), é descrito e analisado. Dentre os algoritmos de otimização disponíveis no pacote CVX, o que se demonstrou mais eficaz foi o Mosek.

Ao se realizarem otimizações variando o valor do coeficiente de ponderação $\mu$, percebeu-se que os resultados nos objetivos de tempo final $T_{f}$ e de energia total $E_{t}$, quando traçados em um gráfico $T_{f} \times E_{t}$, apresentam comportamento exponencial. Esta observação motivou a perspectiva de se encontrar um ponto ótimo dentre todos na Fronteira de Pareto. 
Um algoritmo foi proposto para que um usuário possa entrar apenas com um valor de ponderação entre o tempo final e a energia total e, dado o modelo do robô e a curva por onde ele deve passar, o algoritmo gere os sinais de tensão e de velocidade ótimos. 



\section{PLANEJAMENTO DE CAMINHO}

O Capítulo 3 apresenta uma proposta de planejamento de trajetória para um caminho dado, que é definido pela camada de Planejamento de Caminho na arquitetura NHC. Em uma missão onde o robô móvel deve partir de uma postura específica e chegar em outra em um ambiente livre de obstáculos, a solução ótima encontrada na literatura são as Curvas de Dubins. Este Capítulo discute a utilização desta classe de curvas na proposta de planejamento de caminho apresentada.

\subsection{CURVAS DE DUBINS}

Sejam dois pontos no plano $\mathbf{p}_{0}=\left[\begin{array}{ll}x_{0} & y_{0}\end{array}\right]^{T}$ e $\mathbf{p}_{F}=\left[\begin{array}{ll}x_{F} & y_{F}\end{array}\right]^{T}$ que definem, respectivamente, a posição inicial e final do robô. A cada um dos pontos é associado um ângulo de orientação - $\theta_{0}$ e $\theta_{F}$, respectivamente - que definem a direção do movimento nestes pontos de interesse. A combinação dos pontos e de seus ângulos definem a postura inicial $\boldsymbol{\xi}_{0}=\left[\begin{array}{ll}\mathbf{p}_{\mathbf{0}} & \theta_{0}\end{array}\right]^{T}=\left[\begin{array}{lll}x_{0} & y_{0} & \theta_{0}\end{array}\right]^{T}$ e a postura final $\boldsymbol{\xi}_{F}=\left[\begin{array}{ll}\mathbf{p}_{\mathbf{F}} & \theta_{F}\end{array}\right]^{T}=\left[\begin{array}{lll}x_{F} & y_{F} & \theta_{F}\end{array}\right]^{T}$ do robô no espaço de configuração.

Então, dadas as posturas inicial $\boldsymbol{\xi}_{0}$ e final $\boldsymbol{\xi}_{F}$ de um robô, respectivamente, a tarefa de planejamento de caminho é encontrar a geodésica (o menor caminho) entre estas configurações que possua uma curvatura limitada por $1 / \rho$, onde $\rho$ é o mínimo raio de uma curva em seu movimento.

A solução para este problema é apresentada por Dubins (1957). Em seu trabalho ele mostra que existe uma família de caminhos possíveis que levam de uma postura inicial $\boldsymbol{\xi}_{0}$ para uma postura final $\boldsymbol{\xi}_{F}$, na qual cada elemento desta família é composto por três segmentos de caminho distintos, apresentando a sequência $C C C$ ou $C S C$, onde o segmento do tipo $C$ (do inglês circle ou círculo) é um arco de raio $\rho$, e o segmento do tipo $S$ (straight ou reta) é um segmento de reta. Cada arco $C$ possui duas opções - $L$ para a esquerda (left) ou $R$ para a direita (right). Então, o Conjunto de Dubins $D$ inclui seis classes de caminhos admissíveis: $D=\{L S L, R S R, R S L, L S R, R L R, L R L\}$.

Além disso, Dubins afirma que apenas caminhos com a sequência CSC com arcos de raio $\rho$ podem ser candidatos à geodésica. Portanto, dentro da família de caminhos possíveis, apenas quatro classes de curvas podem ser candidatas à geodésica - as classes curvas $L S L, R S R, R S L$ e $L S R$ com raio de curvatura $\rho$. Exemplos dessas curvas são ilustrados na Figura 35. Algoritmos para a construção destas candidatas, a partir das posturas inicial e final, podem ser encontrados em (LAVALLE, 2006; CHO; RYEU, 2006; GIESE, 2012; LUGO-CARDENAS et al., 2014). 


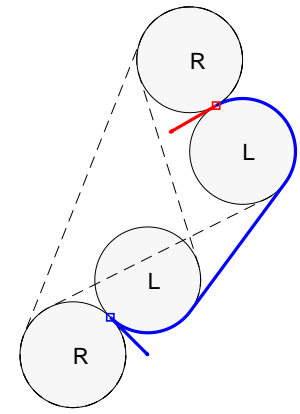

(a) LSL

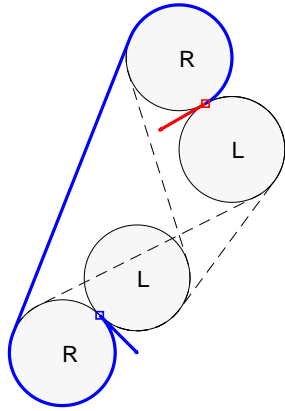

(b) RSR

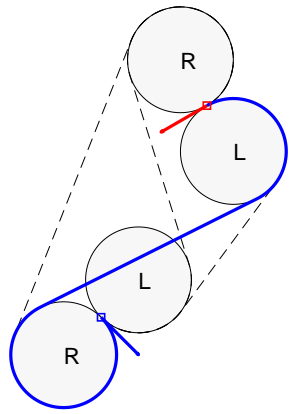

(c) RSL

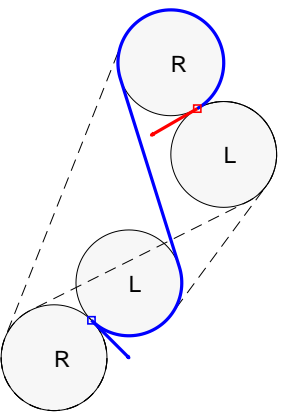

(d) LSR

Figura 35 - Curvas de Dubins do tipo $C S C$ candidatas à geodésica. Fonte: o autor.

Para usar os resultados de Dubins e definir dentre as candidatas o caminho mais curto, calculam-se os comprimentos de todos os arcos e segmentos de retas e então se escolhe o menor dos caminhos calculados. Porém, o tempo necessário para estes cálculos pode ser um gargalo em aplicações em tempo real.

Shkel e Lumelsky (2001) apresentam um esquema que permite selecionar o menor caminho de um conjunto Dubins diretamente, sem o usual cálculo de comprimento de cada um dos seus elementos. Este esquema é baseado no fato de que elementos do conjunto Dubins podem ser classificados em 16 grupos de equivalência, baseados nos quadrantes dos ângulos inicial e final. Os autores provam que dados os quadrantes dos ângulos inicial e final, e uma função de chaveamento específica para cada classe de equivalência, uma tabela de decisão define qual tipo das Dubins possui o menor caminho.

\subsection{OTIMIZAÇÃO DE TRAJETÓRIA EM CURVAS DUBINS}

A proposta deste Capítulo é a otimização da trajetória do robô móvel proposta no Capítulo 3 sobre uma curva Dubins. Tanto o algoritmo de geração de candidatas à geodésica (GIESE, 2012) quanto a seleção da curva ótima (SHKEL; LUMELSKY, 2001) são apresentados por Walker (2008) em um algoritmo de código aberto em Matlab ${ }^{\circledR}$ cuja função

$$
\boldsymbol{\xi}_{d}=\operatorname{dubins}\left(\boldsymbol{\xi}_{0}, \boldsymbol{\xi}_{F}, \rho, N\right)
$$

retorna a matriz $\boldsymbol{\xi}_{d} \in \mathbb{R}^{(N+1) \times 3}, \boldsymbol{\xi}_{d}=\left[\begin{array}{ll}\boldsymbol{\xi}_{0} & \left\{\boldsymbol{\xi}_{d i}\right\}_{i=1}^{N}\end{array}\right]^{T}, \boldsymbol{\xi}_{N}=\boldsymbol{\xi}_{F}$, que representa os $N+1$ vetores de posturas do robô na geodésica Dubins.

Como exemplo, sejam as posturas inicial e final do robô $\boldsymbol{\xi}_{0}=\left[\begin{array}{lll}0 & 0 & -\pi / 4\end{array}\right]^{T}$ e $\boldsymbol{\xi}_{F}=\left[\begin{array}{lll}2 & 4 & 7 \pi / 6\end{array}\right]^{T}$, com um raio de curvatura $\rho=1 \mathrm{~m}$. As Figuras 36, 37, 38 e 39 ilustram os resultados da linearização discreta apresentada na Seção 3.3 da geodésica Dubins para $N=50, N=100, N=200$ e $N=500$, respectivamente. 


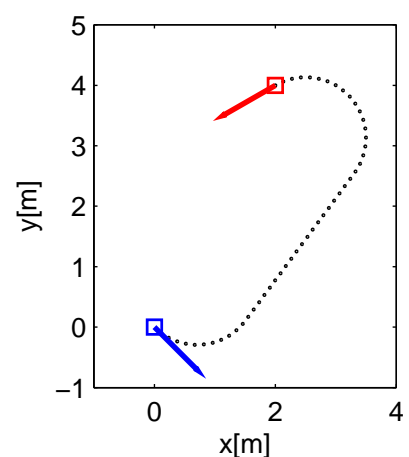

(a)
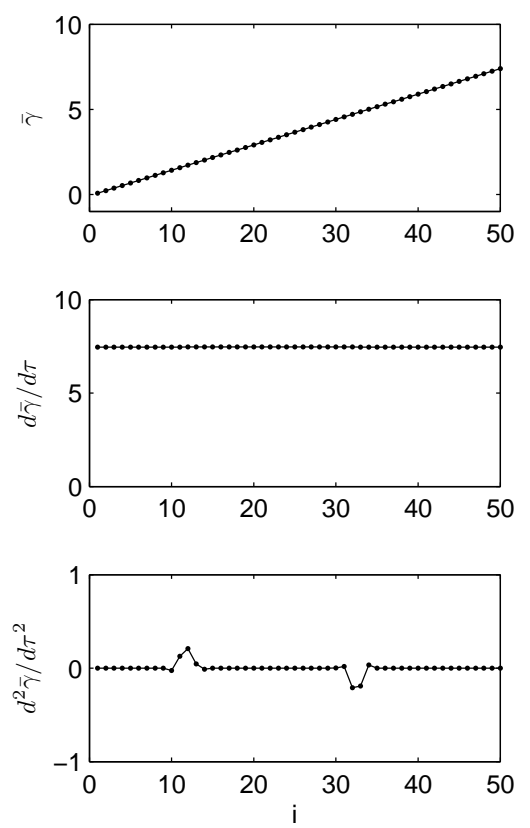

(b)
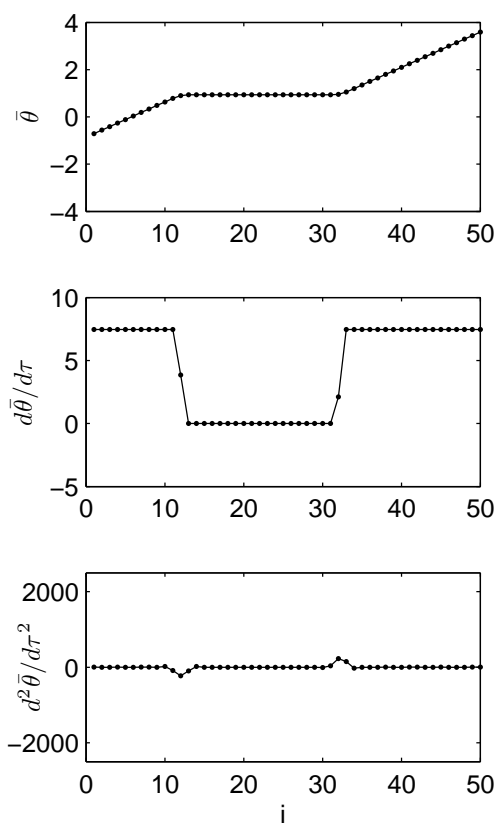

(c)

Figura 36 - Curva Dubins e suas derivadas para $N=50$

Fonte: o autor
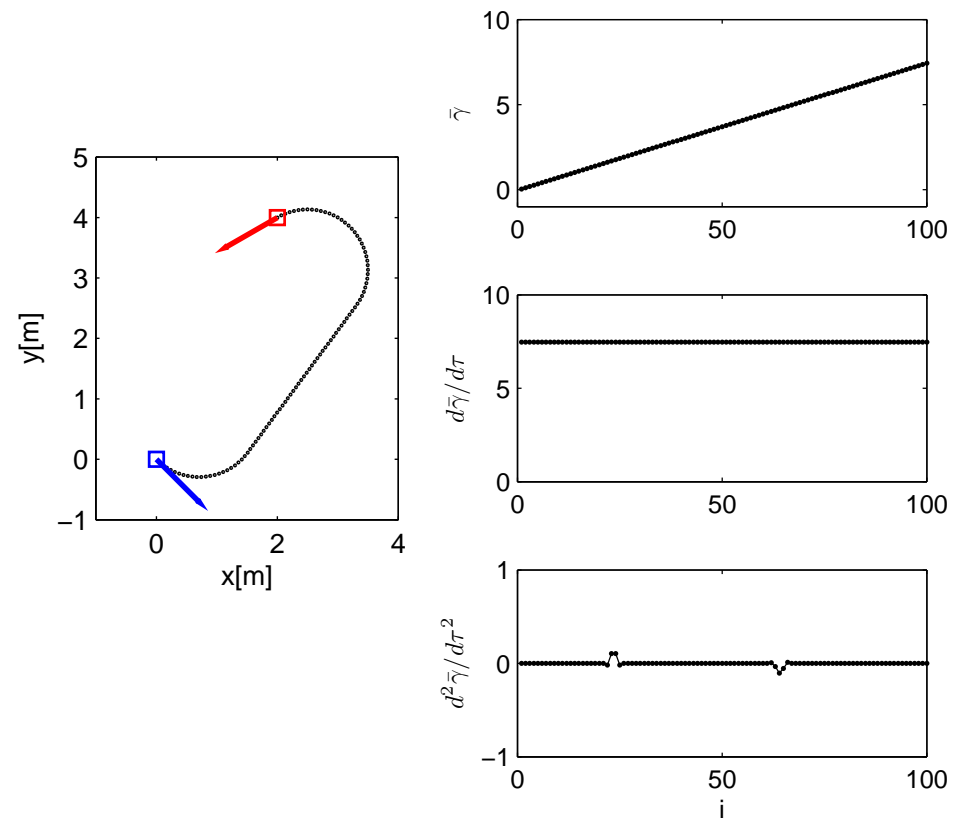

(a)
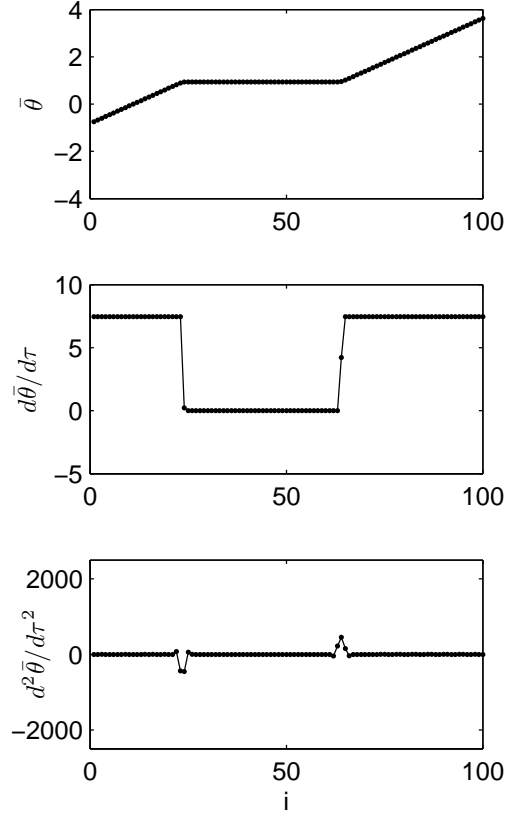

(c)

Figura 37 - Curva Dubins e suas derivadas para $N=100$

Fonte: o autor. 


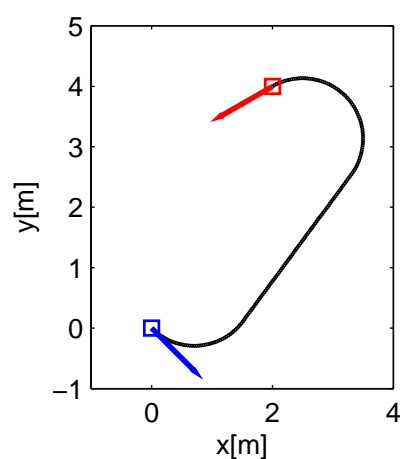

(a)
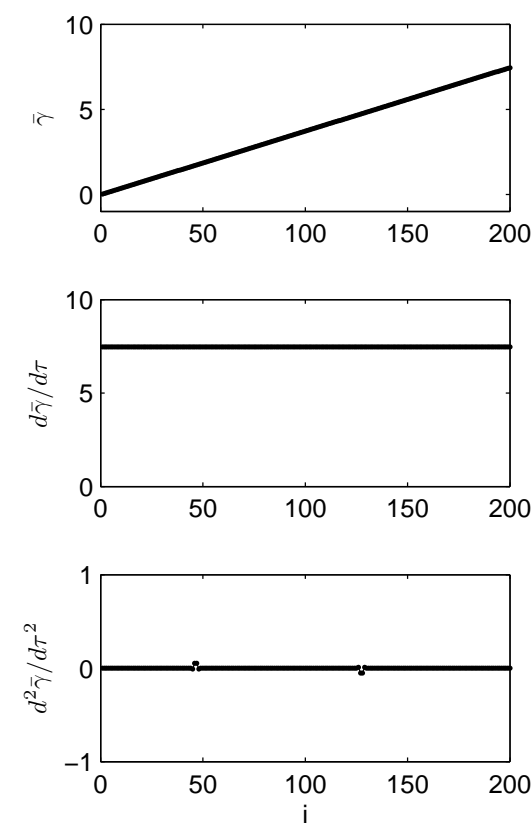

(b)
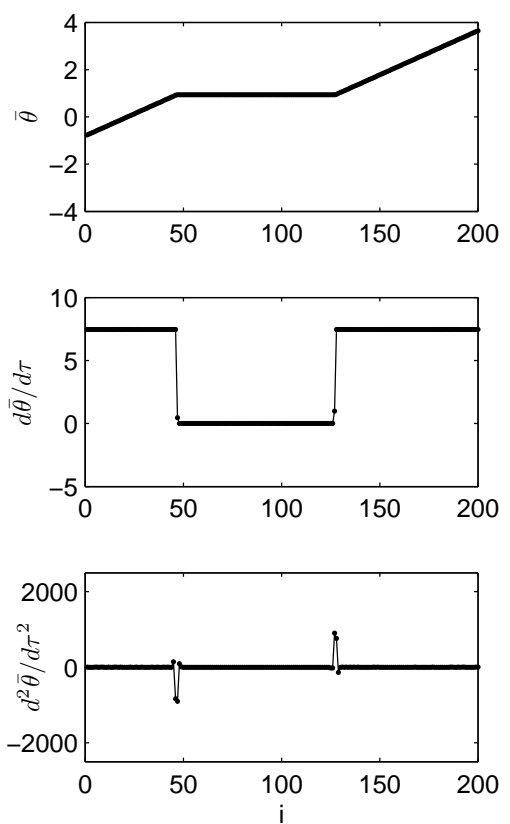

(c)

Figura 38 - Curva Dubins e suas derivadas para $N=200$

Fonte: o autor.
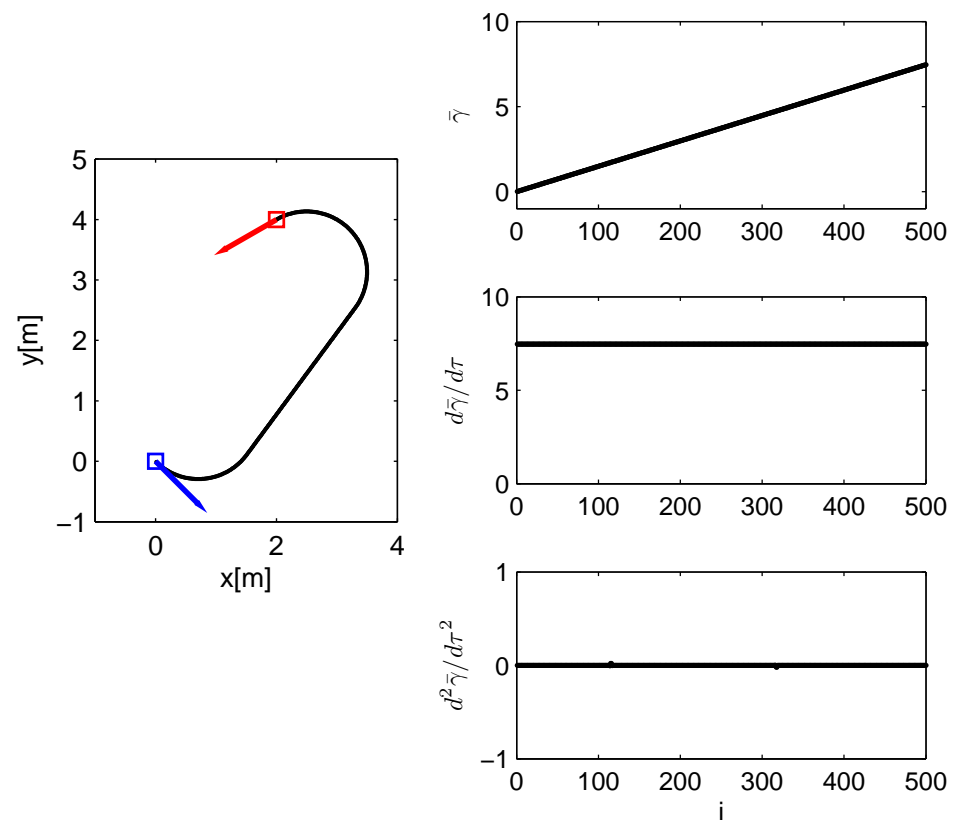

(a)
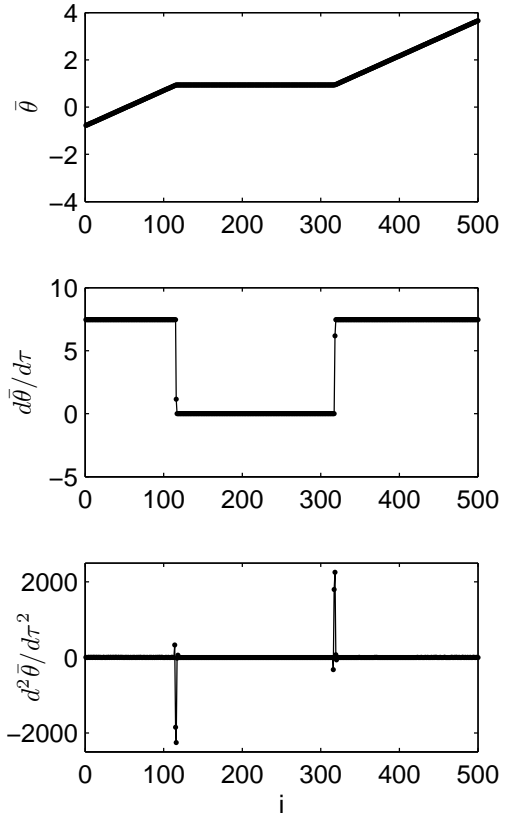

(c)

Figura 39 - Curva Dubins e suas derivadas para $N=500$

Fonte: o autor. 
As subfiguras (a) ilustram a geodésica Dubins no plano com as posturas inicial e final representadas por setas. As subfiguras (b) ilustram a discretização no ponto médio da função avanço $\bar{\gamma}_{i}, i=1, \ldots, N$ e suas derivadas de primeira e segunda ordens; enquanto as subfiguras (c) ilustram a discretização no ponto médio da função orientação $\bar{\theta}_{i}, i=1, \ldots, N$, assim como suas derivadas.

O comprimento total da curva geodésica Dubins contínua é igual a $\gamma(\tau)=\gamma(1)$, e seu avanço é constante. Assim, a derivada de primeira ordem do avanço é constante $\gamma^{\prime}(\tau)=\gamma(\tau)$, e a derivada de segunda ordem do avanço é $\gamma^{\prime \prime}=0, \tau=[0,1]$.

A curva geodésica Dubins contínua possui três segmentos CSC. Nos segmentos do tipo $C$, a derivada de primeira ordem da função orientação é constante $\theta^{\prime}=\gamma / \rho$ para arcos para a esquerda $(L)$ e $\theta^{\prime}=-\gamma / \rho$ para arcos para a direita $(R)$; portanto, a sua derivada de segunda ordem é $\theta^{\prime \prime}=0$, tanto nos segmentos do tipo $C$ quanto no segmento do tipo $S$.

Nos pontos de transição entre os segmentos, i.e., no ponto $\mathbf{p}_{d}\left(\tau_{C R}\right)$ em que o primeiro arco tangencia com o segmento de reta, e em $\mathbf{p}_{d}\left(\tau_{R C}\right)$, onde este segmento tangencia o segundo arco, existe uma descontinuidade na derivada segunda da função de orientação. No exemplo dado, estes pontos estão em $\mathbf{p}_{d}\left(\tau_{C R}\right)=\mathbf{p}_{d}(0,231)=\left[\begin{array}{ll}1,515 & 0,117\end{array}\right]^{T}$ e em $\mathbf{p}_{d}\left(\tau_{R C}\right)=\mathbf{p}_{d}(0,635)=\left[\begin{array}{ll}3,307 & 2,548\end{array}\right]^{T}$.

O efeito da descontinuidade pode ser percebido na subfigura 39c, com a discretização em $N=500$, em que a derivada segunda calculada pelo algoritmo de linearização possui picos em $\bar{\theta}_{116}^{\prime \prime}=-2256 \mathrm{rad}$ e $\bar{\theta}_{318}^{\prime \prime}=2256 \mathrm{rad}$.

Como resultado, a matriz $\overline{\mathbf{s}}^{\prime \prime} \in \mathbb{R}^{N \times 2}, \overline{\mathbf{s}}^{\prime \prime}=\left[\left\{\overline{\mathbf{s}}_{\mathbf{i}}\right\}_{i=1}^{N}\right]^{T}$ é mal-condicionada em norma 2. No exemplo apresentado, para $N=500$, tem-se que seu número de condicionamento $^{1}$ em norma 2 é $\operatorname{cond}\left(\overline{\mathbf{s}}^{\prime \prime}, 2\right)=8,75 \times 10^{5}$.

O mal-condicionamento em norma 2 da matriz $\overline{\mathbf{s}}^{\prime \prime}$ reflete no sistema de equações do Problema 3.4, tornando o sistema igualmente mal-condicionado. Como consequência, o algoritmo de otimização Mosek utilizado com o pacote CVX do Matlab ${ }^{\circledR}$ retorna o erro:

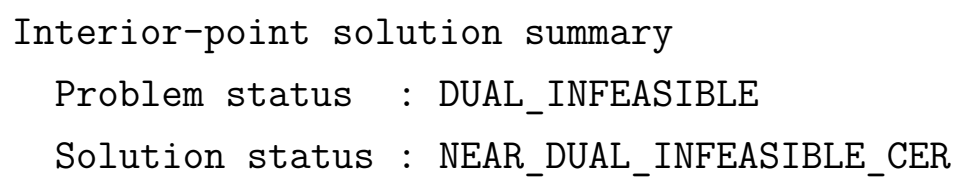

Mosek error: MSK_RES_TRM_STALL（）

Isto significa que o problema de mal-condicionamento da matriz impede que o otimizador possa fazer um progresso razoável e bloqueia seu processo (MOSEK, 2017)

1 Calculado no Matlab $^{\circledR}$ 


\subsection{CURVAS DUBINS SUAVES}

O algoritmo de planejamento de trajetória apresentado no Capítulo 3 tem como pré-requisito que o caminho seja descrito por uma curva cuja segunda derivada seja contínua, i.e., que a curva seja de classe $C^{2}$. Isto se deve ao fato de que as equações de restrição relativas à dinâmica do sistema (Eq. 3.69) possuem termos derivativos de segunda ordem da curva discretizada em pontos médios descritos por $\overline{\mathbf{s}}_{i}^{\prime \prime}$.

As geodésicas de Dubins são a solução geometricamente ótima para o problema de planejamento de caminho, mas não possuem derivada segunda contínua em todo o seu percurso. Para utilizar esta classe de curvas como caminho no algoritmo de planejamento de trajetória apresentado, propõe-se desenvolver um algoritmo para a suavização dessas curvas.

Surgiram algumas ideias para realizar esta suavização. Primeiramente tentou-se filtrar a série de derivadas de primeira ordem da orientação $d \bar{\theta} / d \tau$; porém, a suavização desta derivada, apesar de eliminar a descontinuidade da derivada de segunda ordem, não garante a reconstituição da curva original. A segunda ideia foi o deslocamento geométrico de alguns pontos $b p_{i}$ na proximidade dos pontos de descontinuidade $\mathbf{p}_{d}\left(\tau_{C R}\right)$ e $\mathbf{p}_{d}\left(\tau_{R C}\right)$, utilizando alguma função específica; esta ideia também demonstrou-se inadequada pois outras descontinuidades nas derivadas apareceram entre a função de suavização e a curva Dubins original. Seria necessário então encontrar uma função que pudesse suavizar a geodésica em toda sua extensão.

Seja o exemplo ilustrado na Seção 4.1, $\boldsymbol{\xi}_{0}=\left[\begin{array}{lll}0 & 0 & -\pi / 4\end{array}\right]^{T}$ e $\boldsymbol{\xi}_{F}=\left[\begin{array}{lll}2 & 4 & 7 \pi / 6\end{array}\right]^{T}$, com um raio de curvatura $\rho=1 \mathrm{~m}$, e a geodésica Dubins, dada pela matriz $\boldsymbol{\xi}$ composta por $N+1$ posturas. Esta curva possui um comprimento de 7,47m. Seja também uma curva Dubins "relaxada", construída pela função

$$
\boldsymbol{\xi}_{w}=\operatorname{dubins}\left(\boldsymbol{\xi}_{0}, \boldsymbol{\xi}_{F}, \rho, n\right),
$$

onde $n \ll N$. Arredondando-se o comprimento da curva para $7 \mathrm{~m}$, faz-se $n=7$. Então, a função retorna um conjunto de $n+1$ posturas dadas pela matriz $\boldsymbol{\xi}_{w}=\left[\begin{array}{lll}\mathbf{x}_{w} & \mathbf{y}_{w} & \boldsymbol{\theta}_{w}\end{array}\right]^{T}$, onde os vetores

$$
\mathbf{x}_{w}=\left[\begin{array}{r}
0,0000 \\
0,9849 \\
1,7573 \\
2,3912 \\
3,0251 \\
3,4992 \\
3,0169 \\
2,0000
\end{array}\right] \quad e \quad \mathbf{y}_{w}=\left[\begin{array}{r}
0,0000 \\
-0,2535 \\
0,4457 \\
1,3038 \\
2,1619 \\
3,0947 \\
3,9900 \\
4,0000
\end{array}\right]
$$

definem $n+1$ pontos de controle (ou waypoints) a cada aproximadamente $1 \mathrm{~m}$ no plano. 
A Figura 40 ilustra a geodésica Dubins $\mathbf{p}_{d i}=\left[\begin{array}{ll}x_{d i} & y_{d i}\end{array}\right]^{T}$ gerada com $N=500$, em tracejado, e os pontos de controle $\mathbf{p}_{w i}=\left[\begin{array}{ll}x_{w i} & y_{w i}\end{array}\right]^{T}$ gerados com $n=7$. Na subfigura 40a são mostradas as curvas no plano, e na subfigura 40b as componentes cartesianas das curvas parametrizadas por $n \tau, \tau=[0,1]$.

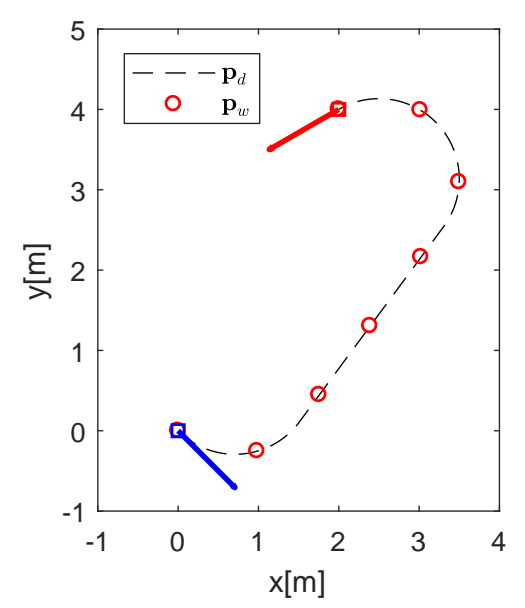

(a)

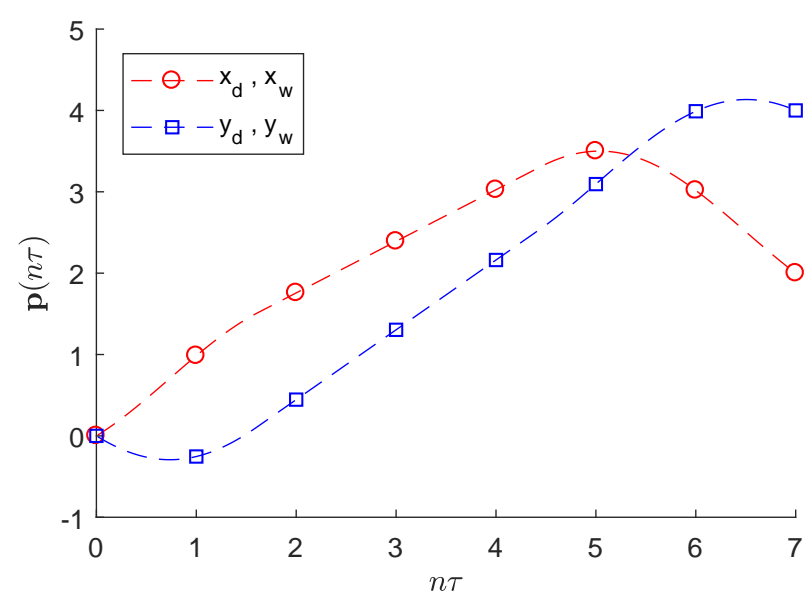

(b)

Figura 40 - Curva Dubins com $n+1=7$ waypoints (a) no plano cartesiano e (b) suas coordenadas parametrizadas por $n \tau$

Fonte: o autor.

A idéia principal é interpolar geometricamente uma curva entre os waypoints dados por $\mathbf{p}_{w i}=\left[\begin{array}{ll}x_{w i} & y_{w i}\end{array}\right]^{T}, i=0, \ldots, n$, com uma curva classe $C^{2}$. Propõe-se usar uma interpolação por splines cúbicas (BURDEN; FAIRES, 2010), que são curvas por partes, onde cada parte é uma função cúbica de um parâmetro independente. Por serem funções cúbicas, cada parte é por si só duplamente diferenciável. Restrições na junção entre as partes da curva impõem que a curva completa também seja duplamente diferenciável; e restrições nas derivadas nos extremos impõem que a orientação do robô no início e no fim da curva sejam respeitadas.

\subsubsection{Interpolação por Splines Cúbicas Grampeadas}

Seja uma função $w(\tau)$ definida em $\left[\tau_{0}, \tau_{n}\right]$ dada por um conjunto de $n+1$ pares de pontos $\left(\tau_{i}, w_{i}\right), i=0, \ldots, n, \tau_{0}<\tau_{1}<\ldots<\tau_{n}$. Uma spline cública interpolante $S$ para $w$ é uma função por partes do tipo

$$
S(\tau)=\left\{\begin{array}{cl}
a_{0}+b_{0}\left(\tau-\tau_{0}\right)+c_{0}\left(\tau-\tau_{0}\right)^{2}+d_{0}\left(\tau-\tau_{0}\right)^{3} & , \tau_{0} \leq \tau \leq \tau_{1} \\
a_{1}+b_{1}\left(\tau-\tau_{1}\right)+c_{1}\left(\tau-\tau_{1}\right)^{2}+d_{1}\left(\tau-\tau_{1}\right)^{3} & , \tau_{1} \leq \tau \leq \tau_{2} \\
\vdots & \\
a_{n-1}+b_{n-1}\left(\tau-\tau_{n-1}\right)+c_{n-1}\left(\tau-\tau_{n-1}\right)^{2}+d_{n-1}\left(\tau-\tau_{n-1}\right)^{3} & , \tau_{n-1} \leq \tau<\tau_{n}
\end{array}\right.
$$


ou, na forma genérica,

$$
S_{j}\left(\tau_{j}\right)=a_{j}+b_{j}\left(\tau-\tau_{j}\right)+c_{j}\left(\tau-\tau_{j}\right)^{2}+d_{j}\left(\tau-\tau_{j}\right)^{3}
$$

$j=0, \ldots,(n-1), \tau_{j} \leq \tau \leq \tau_{j+1}$. As $n$ partes $S_{j}$ dadas pelo conjunto de Equações (4.4), ilustradas na Figura 41, são funções cúbicas de $\tau$, e seu conjunto possui $4 n$ coeficientes $a_{j}$, $b_{j}, c_{j}$ e $d_{j}, j=0, \ldots,(n-1)$ a serem encontrados.

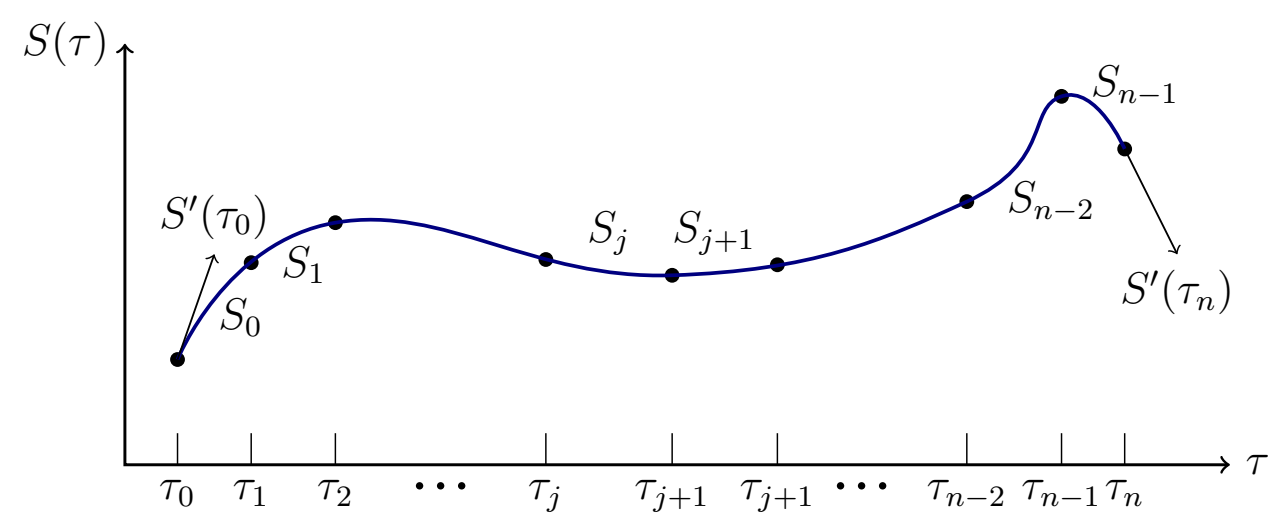

Figura 41 - Interpolação spline cúbica grampeada. A curva é composta por $n$ partes $S_{j}$, e seus extremos com derivadas definidas.

Fonte: adaptado de Burden e Faires (2010).

Para tanto, algumas condições são impostas:

- A função spline cúbica interpola os pontos definidos por $w$, i.e,

$$
S_{j}\left(\tau_{j}\right)=w\left(\tau_{j}\right) \text { e } S_{j}\left(\tau_{j+1}\right)=w\left(\tau_{j+1}\right), j=0, \ldots,(n-1)
$$

- A função spline cúbica é contínua, i.e,

$$
S_{j+1}\left(\tau_{j+1}\right)=S_{j}\left(\tau_{j+1}\right), j=0, \ldots,(n-2)
$$

- A função spline cúbica tem sua primeira derivada contínua, i.e,

$$
S_{j+1}^{\prime}\left(\tau_{j+1}\right)=S_{j}^{\prime}\left(\tau_{j+1}\right), j=0, \ldots,(n-2)
$$

- A função spline cúbica tem sua segunda derivada contínua, i.e,

$$
S_{j+1}^{\prime \prime}\left(\tau_{j+1}\right)=S_{j}^{\prime \prime}\left(\tau_{j+1}\right), j=0, \ldots,(n-2)
$$

- As derivadas nos extremos da spline são determinadas (grampeadas), i.e,

$$
S^{\prime}\left(\tau_{0}\right)=S_{0}^{\prime} \text { e } S^{\prime}\left(\tau_{n}\right)=S_{F}^{\prime}
$$


A condição (4.6) possui $n+1$ equações. As condições (4.7), (4.8) e (4.9) impõem cada qual $n-1$ equações. Com as duas condições (4.10), tem-se $4 n$ equações suficientes para se encontrar os coeficientes das funções parciais presentes em (4.4).

Da forma genérica 4.5 , tem-se que $S_{j}\left(\tau_{j}\right)=a_{j}=w\left(\tau_{j}\right)$. Aplicando-se a condição (4.7), obtém-se

$$
S_{j+1}\left(\tau_{j+1}\right)=a_{j+1}=S_{j}\left(\tau_{j+1}\right)=a_{j}+b_{j}\left(\tau_{j+1}-\tau_{j}\right)+c_{j}\left(\tau_{j+1}-\tau_{j}\right)^{2}+d_{j}\left(\tau_{j+1}-\tau_{j}\right)^{3},
$$

para cada $j=0, \ldots,(n-2)$. Usando a notação $h_{j}=\tau_{j+1}-\tau_{j}, j=0, \ldots,(n-1)$, escreve-se

$$
a_{j+1}=a_{j}+b_{j} h_{j}+c_{j} h_{j}^{2}+d_{j} h_{j}^{3}
$$

$j=0, \ldots,(n-1)$.

A primeira derivada da spline (4.5) é dada por

$$
S_{j}^{\prime}\left(\tau_{j}\right)=b_{j}+2 c_{j}\left(\tau-\tau_{j}\right)+3 d_{j}\left(\tau-\tau_{j}\right)^{2},
$$

que implica $S_{j}^{\prime}\left(\tau_{j}\right)=b_{j}, j=0, \ldots,(n-1)$. Pela condição (4.8), obtém-se

$$
S_{j+1}^{\prime}\left(\tau_{j+1}\right)=b_{j+1}=S_{j}^{\prime}\left(\tau_{j+1}\right)=b_{j}\left(\tau_{j+1}-\tau_{j}\right)+2 c_{j}\left(\tau_{j+1}-\tau_{j}\right)+3 d_{j}\left(\tau_{j+1}-\tau_{j}\right)^{2},
$$

para cada $j=0, \ldots,(n-2)$, ou

$$
b_{j+1}=b_{j} h_{j}+2 c_{j} h_{j}+3 d_{j} h_{j}^{2},
$$

$j=0, \ldots,(n-1)$.

A segunda derivada da spline (4.5) é dada por

$$
S_{j}^{\prime \prime}\left(\tau_{j}\right)=2 c_{j}+6 d_{j}\left(\tau-\tau_{j}\right),
$$

que implica $S_{j}^{\prime \prime}\left(\tau_{j}\right)=2 c_{j}, j=0, \ldots,(n-1)$. Aplicando a condição $(4.9)$, tem-se

$$
S_{j+1}^{\prime \prime}\left(\tau_{j+1}\right)=2 c_{j+1}=S_{j}^{\prime \prime}\left(\tau_{j+1}\right)=2 c_{j}+6 d_{j}\left(\tau_{j+1}-\tau_{j}\right),
$$

para cada $j=0, \ldots,(n-2)$, ou

$$
c_{j+1}=c_{j}+3 d_{j} h_{j}
$$

$j=0, \ldots,(n-1)$.

Isolando $d_{j}$ da equação (4.15),

$$
d_{j}=\frac{c_{j-1}-c_{j}}{3 h_{j}}
$$

, e substituindo em (4.11) e (4.13), tem-se respectivamente:

$$
\begin{aligned}
& a_{j+1}=a_{j}+b_{j} h_{j}+\frac{h_{j}^{2}}{3}\left(2 c_{j}+c_{j+1}\right), \\
& b_{j+1}=b_{j}+h_{j}\left(c_{j}+c_{j+1}\right) .
\end{aligned}
$$


Isolando $b_{j}$ na equação $(4.17)$, tem-se

$$
b_{j}=\frac{1}{h_{j}}\left(a_{j+1}-a j\right)-\frac{h_{j}}{3}\left(2 c_{j}+c_{j+1}\right),
$$

e reduzindo o índice, tem-se

$$
b_{j-1}=\frac{1}{h_{j-1}}\left(a_{j}-a j-1\right)-\frac{h_{j-1}}{3}\left(2 c_{j-1}+c_{j}\right) .
$$

A equação (4.18) com o índice reduzido,

$$
b_{j}=b_{j-1}+h_{j-1}\left(c_{j-1}+c_{j}\right),
$$

e substituindo os termos $b_{j}$ e $b_{j-1}$ pelas equações (4.19) e (4.20), respectivamente, com algumas manipulações algébricas, leva a

$$
h_{j-1} c_{j-1}+2\left(h_{j-1}+h_{j}\right) c_{j}+h_{j} c_{j+1}=\frac{3}{h_{j}}\left(a_{j+1}-a_{j}\right)-\frac{3}{h_{j-1}}\left(a_{j}-a_{j-1}\right),
$$

$j=0, \ldots,(n-1)$.

Da restrição (4.10), a derivada no limite inferior dada por $S^{\prime}\left(\tau_{0}\right)=S_{0}^{\prime}=b_{0}$. Substituindo em (4.19),

$$
b_{0}=\frac{1}{h_{0}}\left(a_{1}-a 0\right)-\frac{h_{0}}{3}\left(2 c_{0}+c_{1}\right)=S_{0}^{\prime},
$$

ou seja,

$$
2 h_{0} c_{0}+h_{0} c_{1}=\frac{3}{h_{0}}\left(a_{1}-a_{0}\right)-3 S_{0}^{\prime} .
$$

Da mesma forma, pela restrição (4.10), a derivada no limite superior dada por $S^{\prime}\left(\tau_{n}\right)=S_{F}^{\prime}=b_{n}$. De $(4.20)$, tem-se

$$
b_{n}=b_{n-1}+h_{n-1}\left(c_{n-1}+c_{n}\right) .
$$

Substituindo (4.20) no termo $b_{n-1}$, resulta

$$
b_{n}=\frac{1}{h_{n-1}}\left(a_{n}-a_{n-1}\right)-\frac{h_{n-1}}{3}\left(2 c_{n-1}+c_{n}\right)+h_{n-1}\left(c_{n-1}+c_{n}\right)=S_{F}^{\prime} .
$$

Após algumas manipulações algébricas, tem-se

$$
h_{n-1} c_{n-1}+2 h_{n-1} c_{n}=3 S_{F}^{\prime}-\frac{3}{h_{n-1}}\left(a_{n}-a_{n-1}\right) .
$$

As equações recursivas (4.22), em conjunto com as equações que restringem as derivadas nos limites da spline interpolante (4.23) e (4.24), a saber

$$
\left\{\begin{aligned}
2 h_{0} c_{0}+h_{0} c_{1}=\frac{3}{h_{0}}\left(a_{1}-a_{0}\right)-3 S_{0}^{\prime} & j=0 \\
h_{j-1} c_{j-1}+2\left(h_{j-1}+h_{j}\right) c_{j}+h_{j} c_{j+1}=\frac{3}{h_{j}}\left(a_{j+1}-a_{j}\right)-\frac{3}{h_{j-1}}\left(a_{j}-a_{j-1}\right), & 1 \leq j \leq n-2, \\
h_{n-1} c_{n-1}+2 h_{n-1} c_{n}=3 S_{F}^{\prime}-\frac{3}{h_{n-1}}\left(a_{n}-a_{n-1}\right) & j=n-1 .
\end{aligned}\right.
$$


são um sistema linear com coeficientes conhecidos: os espaçamentos entre os nós $h_{j}$, $j=0, \ldots,(n-1)$ e os valores da função $w\left(\tau_{i}\right)$ em cada um dos nós dados por $a_{j}$, $j=0, \ldots, n$, além das derivadas nos limites inferior e superior $S_{0}^{\prime}$ e $S_{F}^{\prime}$, respectivamente. Os termos $c_{j}, j=0, \ldots, n$ são os termos a serem determinados. Para tanto, o sistema (4.25) pode ser escrito como a equação matricial $\mathbf{H c}=\mathbf{f}$, onde

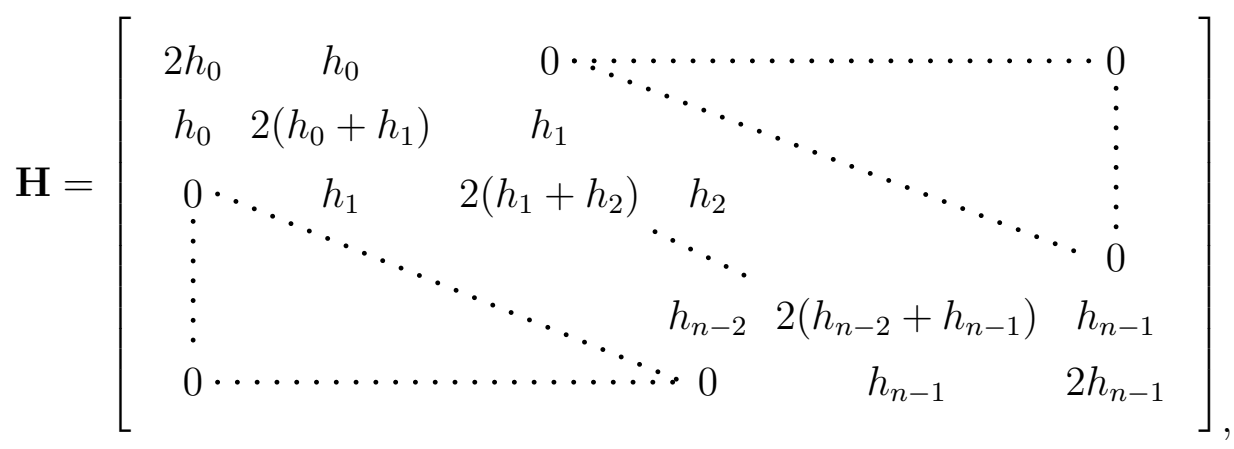

$$
\begin{aligned}
& \mathbf{f}=\left[\begin{array}{c}
\frac{3}{h_{0}}\left(a_{1}-a_{0}\right)-3 S_{0}^{\prime} \\
\frac{3}{h_{1}}\left(a_{2}-a_{1}\right)-\frac{3}{h_{1}}\left(a_{1}-a_{0}\right) \\
\vdots \\
\frac{3}{h_{1}}\left(a_{n}-a_{n-1}\right)-\frac{3}{h_{n-2}}\left(a_{n-1}-a_{n-2}\right) \\
3 S_{F}^{\prime}-\frac{3}{h_{n-1}}\left(a_{n}-a_{n-1}\right)
\end{array}\right], \quad \mathbf{c}=\left[\begin{array}{c}
c_{0} \\
c_{1} \\
\vdots \\
c_{n-1} \\
c_{n}
\end{array}\right] .
\end{aligned}
$$

Como $b_{0}=S^{\prime}(0)$, os termos $b_{j}, j=0, \ldots, n$ podem ser obtidos por recursividade pela Equação 4.21. Como $d_{0}$ pode ser obtido com $a_{0}, b_{0}$ e $c_{0}$, os termos $d_{j}, j=0, \ldots, n$, podem ser obtidos por recursividade pela Equação 4.16 .

\subsubsection{Suavização da Curva Dubins}

O relaxamento da geodésica Dubins, dada no exemplo (Figura 40), gerou um conjunto de $n+1=8$ pontos de controle dados pelos vetores $\mathbf{x}_{w}$ e $\mathbf{y}_{w}$ (Equação 4.3). Estes vetores foram parametrizados por $n \tau=0, \ldots, 7$.

Para cada coordenada cartesiana tem-se um conjunto de $n+1$ pares de pontos $\left(j, x_{w i}\right)$ e $\left(j, y_{w i}\right), j=0, \ldots, n$. Interpolando estes pares de pontos para cada coordenada, pretendem-se obter duas funções spline, $x_{s}(n \tau)$ e $y_{s}(n \tau)$, onde as derivadas inicial e final para cada função são dadas por:

$$
\begin{aligned}
x_{s}^{\prime}(0) & =\frac{x_{d}(1)-x_{d}(0)}{n / N} & x_{s}^{\prime}(n) & =\frac{x_{d}(N)-x_{d}(N-1)}{n / N} \\
y_{s}^{\prime}(0) & =\frac{y_{d}(1)-y_{d}(0)}{n / N} & y_{s}^{\prime}(n) & =\frac{y_{d}(N)-y_{d}(N-1)}{n / N}
\end{aligned}
$$


Como os espaçamentos entre os nós das funções são constantes, $h_{j}=n \tau_{j+1}-n \tau_{j}=1$, $j=0, \ldots,(n-1)$. Resolvendo as equações matriciais $\mathbf{c}_{x}=\mathbf{H}_{x}^{-1} \mathbf{f}_{x}$ e $\mathbf{c}_{y}=\mathbf{H}_{y}^{-1} \mathbf{f}_{y}$, e aplicando as equações recursivas (4.21) e (4.16), tem-se os coeficientes dos polinômios $S_{x j}(n \tau)$ e $S_{y j}(n \tau)$ :

$$
\begin{aligned}
{\left[\begin{array}{llll}
\mathbf{a}_{x} & \mathbf{b}_{x} & \mathbf{c}_{x} & \mathbf{d}_{x}
\end{array}\right]=\left[\begin{array}{rrrr}
0,0000 & 0.7600 & 0.4713 & -0.2465 \\
0.9849 & 0.9633 & -0.2680 & 0.0772 \\
1.7573 & 0.6588 & -0.0365 & 0.0116 \\
2.3912 & 0.6206 & -0.0017 & 0.0150 \\
3.0251 & 0.6621 & 0.0432 & -0.2312 \\
3.4992 & 0.0548 & -0.6505 & 0.1133 \\
3.0169 & -0.9062 & -0.3105 & 0.1998
\end{array}\right] } \\
{\left[\begin{array}{lllrr}
\mathbf{a}_{y} & \mathbf{b}_{y} & \mathbf{c}_{y} & \mathbf{d}_{y}
\end{array}\right]=\left[\begin{array}{rrrr}
0,0000 & -0.7487 & 0.4355 & 0.0596 \\
-0.2535 & 0.3012 & 0.6144 & -0.2164 \\
0.4457 & 0.8809 & -0.0348 & 0.0120 \\
1.3038 & 0.8473 & 0.0013 & 0.0095 \\
2.1619 & 0.8783 & 0.0297 & 0.0248 \\
3.0947 & 1.0121 & 0.1041 & -0.2209 \\
3.9900 & 0.5576 & -0.5587 & 0.0111
\end{array}\right] . }
\end{aligned}
$$

A Figura 42 ilustra os componentes cartesianos da geodésica Dubins original $\mathbf{p}_{d}(n \tau)=\left[\begin{array}{ll}x_{d}(n \tau) & y_{d}(n \tau)\end{array}\right]^{T}$, os waypoints gerados pela geodésica Dubins relaxada $\mathbf{p}_{w}(n \tau)=\left[\begin{array}{ll}x_{w}(n \tau) & y_{w}(n \tau)\end{array}\right]^{T}$ e as curvas por partes que interpolam os waypoints dados por $x_{s}(n \tau)$ e $y_{s}(n \tau)$. A Figura 43 ilustra estas curvas no plano cartesiano.

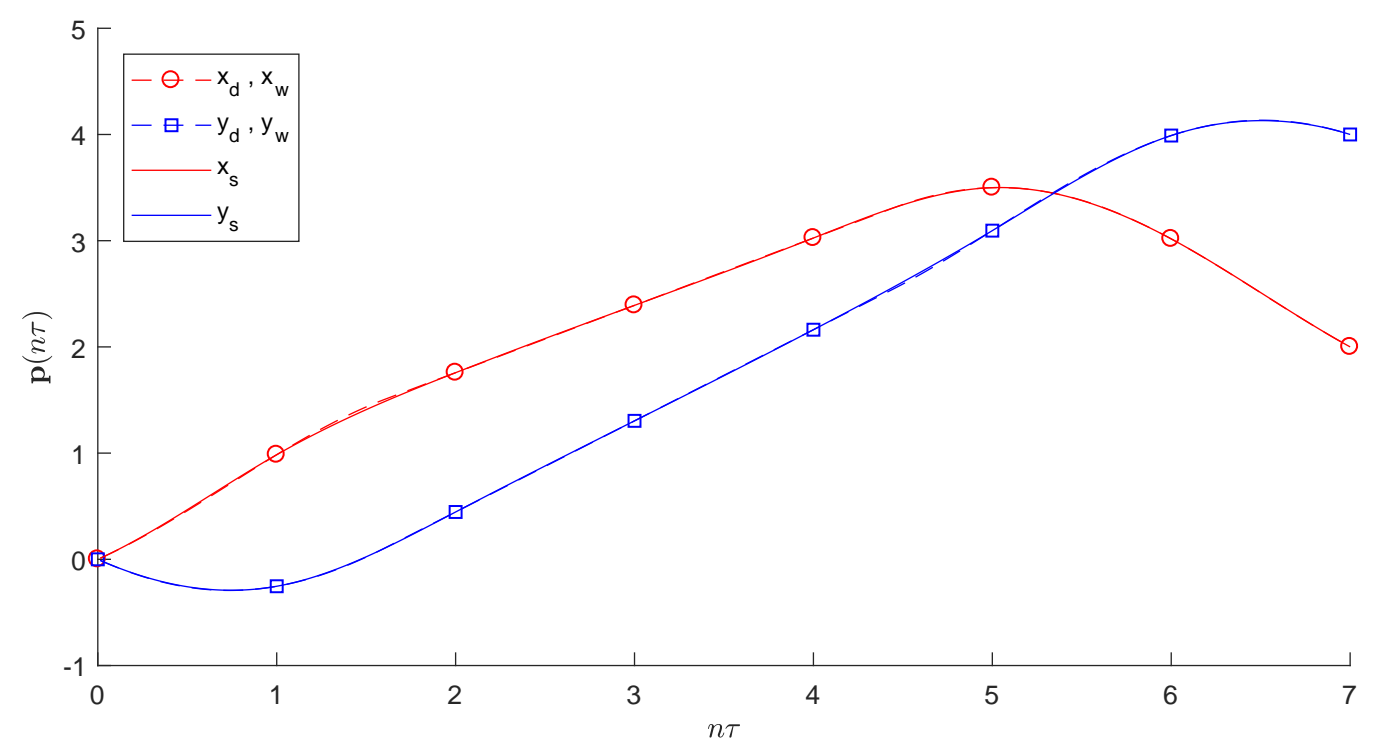

Figura 42 - Componentes cartesianos das Curvas Dubins original e suavizada com os $n+1=8$ waypoints.

Fonte: o autor. 


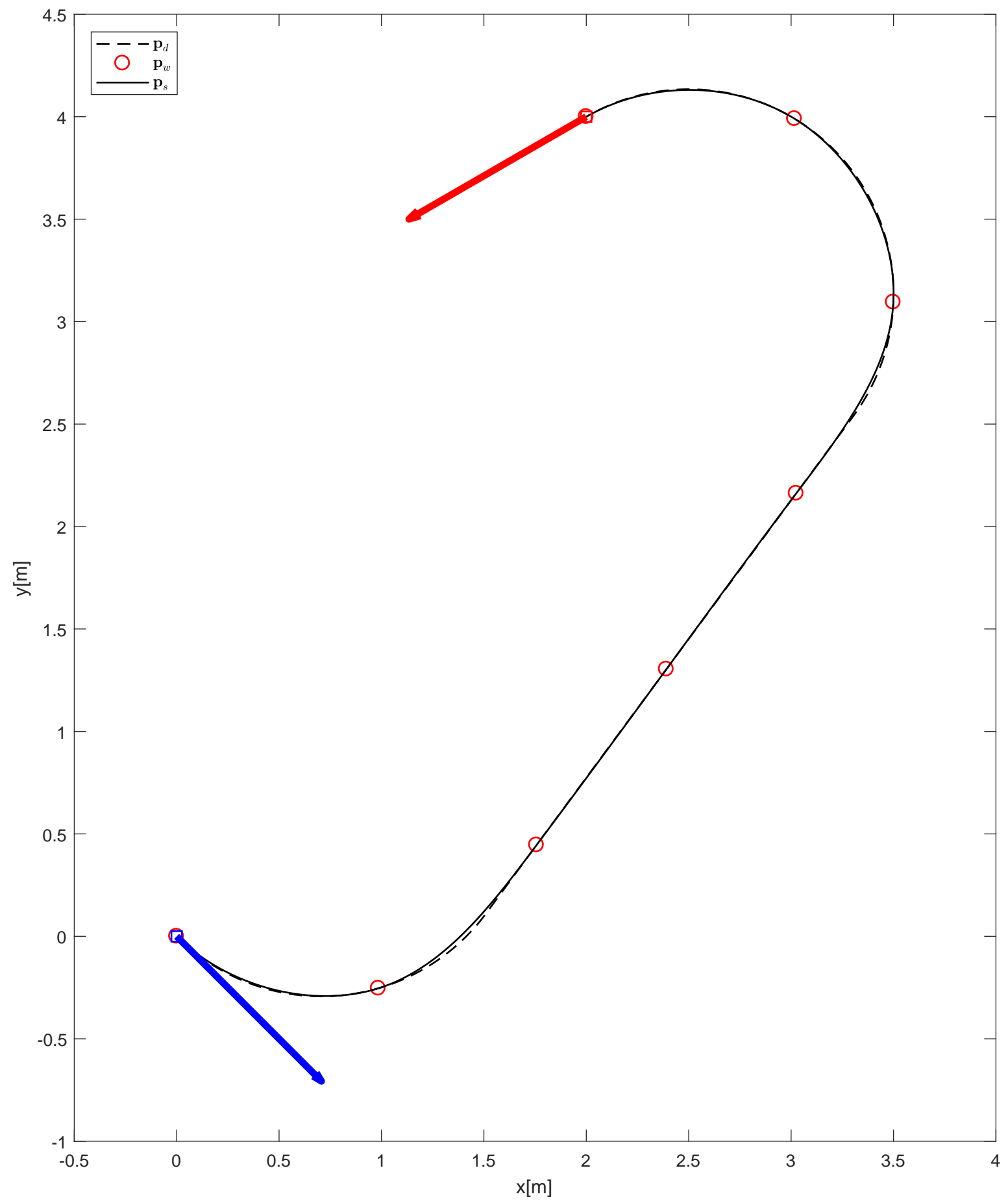

Figura 43 - Curva Dubins suavizada $\left(\mathbf{p}_{s}\right)$. A linha tracejada representa a Curva Dubins original $\left(\mathbf{p}_{d}\right)$ entre as posturas inicial e final, e os círculos vermelhos representam os $n+1=8$ waypoints $\left(\mathbf{p}_{w}\right)$.

Fonte: o autor. 
Observa-se tanto na Figura 42 quanto na Figura 43 que existe uma certa coincidência entre a geodésica Dubins original e a curva spline originada da interpolação entre os waypoints. De fato, o erro máximo entre estas curvas no plano, calculado pela distância entre pontos de ambas as curvas no mesmo intervalo de tempo discreto, i.e., $e_{\max }=$ $\max \left[\sqrt{\left(x_{d i}-x_{s i}\right)^{2}+\left(y_{d i}-y_{s i}\right)^{2}}\right], i=0 \ldots, N$, é relativamente baixo $\left(e_{\max }=0.026 \mathrm{~m}\right)$.

A Figura 44 apresenta os componentes da curva Dubins suavizada em termos da configuração alternativa e suas derivadas - da função avanço em seu ponto médio na Subfigura 44a, e da função orientação em seu ponto médio na Subfigura 44b. Em pontilhado, a representação das mesmas grandezas para a geodésica Dubins original.

Observa-se nos dois gráficos superiores uma certa coincidência entre os componentes de avanço $\gamma$ e de orientação $\theta$ da geodésica e da curva suavizada, assim como observado no plano cartesiano. Esta coincidência não é perfeita, o que se observa nas derivadas de primeira ordem. A observação importante é que a suavização da curva Dubins por uma função spline suave garante a continuidade da segunda derivada da orientação, ilustrada nos gráficos inferiores da Figura 44.
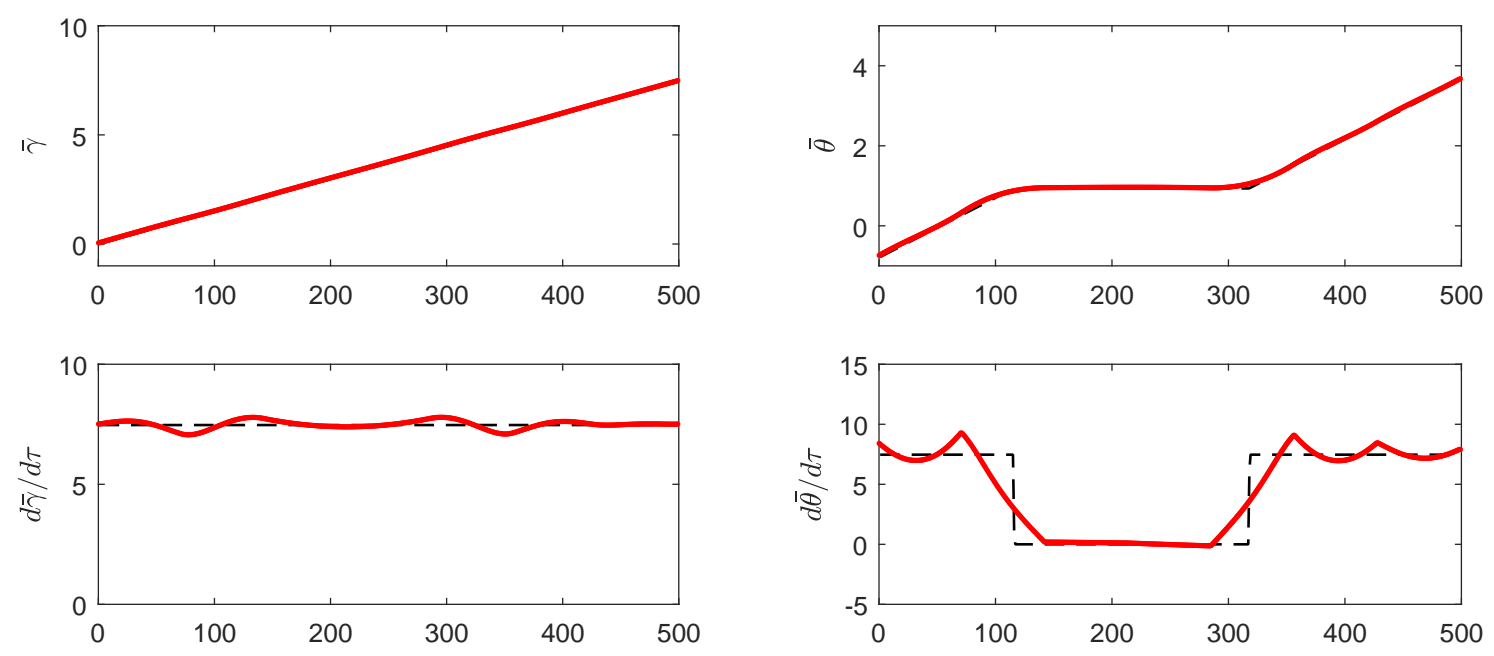

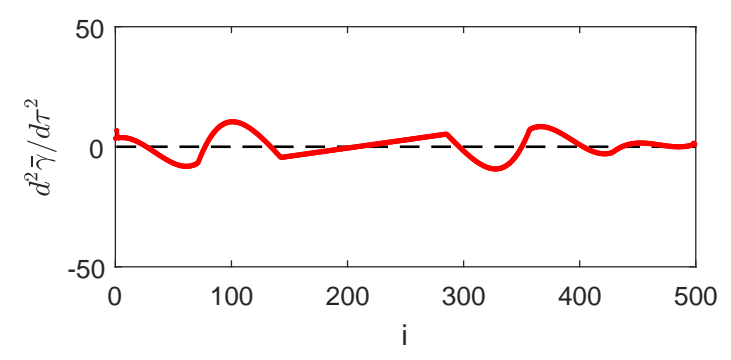

(a)

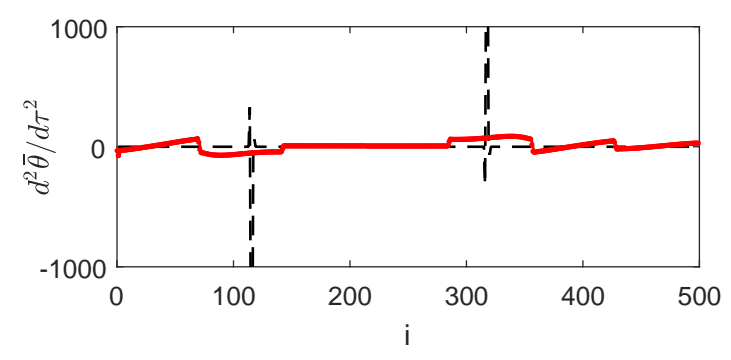

(b)

Figura 44 - Componentes de $\overline{\mathbf{s}}_{i}$ e suas derivadas para a curva Dubins suavizada. A linha pontilhada representa os componentes para a Dubins original.

Fonte: o autor. 
Como resultado, a matriz $\overline{\mathbf{s}}^{\prime \prime} \in \mathbb{R}^{N \times 2}, \overline{\mathbf{s}}^{\prime \prime}=\left[\left\{\overline{\mathbf{s}}_{\mathbf{i}}{ }^{\prime \prime}\right\}_{i=1}^{N}\right]^{T}$, que, para a geodésica Dubins original, tem seu condicionamento em $\operatorname{cond}\left(\overline{\mathbf{s}}^{\prime \prime}, 2\right)=8,75 \times 10^{5}$; para a curva Dubins suavizada por spline, o condicionamento da matriz cai para $\operatorname{cond}\left(\overline{\mathbf{s}}^{\prime \prime}, 2\right)=12,14$.

Como consequência, o algoritmo de otimização Mosek resolve o Problema 3.4, retornando com sucesso os sinais de controle $\mathbf{u}^{*}$ e de velocidade $\mathbf{v}^{*}$. A Figura 45 ilustra a resposta do algoritmo para um coeficiente de ponderação $\mu=100$.
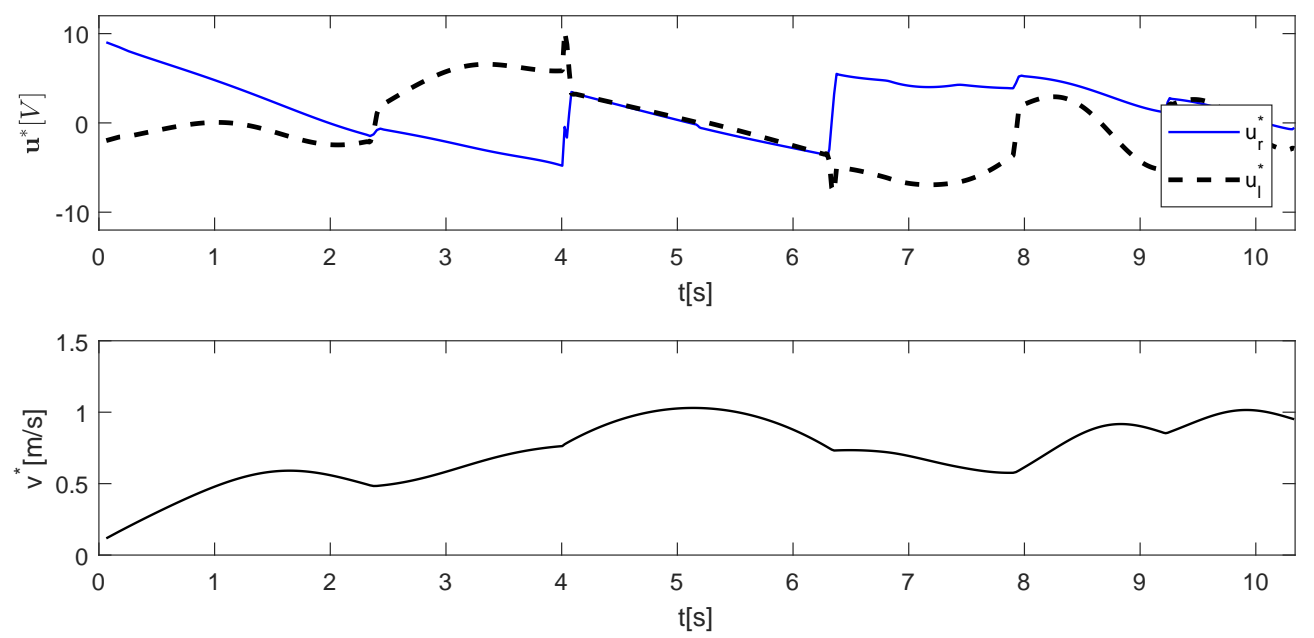

Figura 45 - Resposta do algoritmo SOCP para a curva Dubins suavizada. Fonte: o autor.

A curva Dubins apresentada e suavizada como exemplo é do tipo $L S L$. Como ilustração do método proposto, foram planejadas mais duas curvas.

A primeira curva tem como posturas inicial e final do robô $\boldsymbol{\xi}_{0}=\left[\begin{array}{lll}0 & 0 & \pi\end{array}\right]^{T}$ e $\boldsymbol{\xi}_{F}=\left[\begin{array}{lll}4 & 2 & -\pi / 2\end{array}\right]^{T}$, respectivamente, com um raio de curvatura $\rho=1 \mathrm{~m}$. A geodésica Dubins entre estas duas posturas é uma curva do tipo $R S R$ com um comprimento de $7,87 \mathrm{~m}$. O método de suavização proposto usou $n+1=9$ waypoints, resultando em um erro máximo entre a geodésica original e a curva suavizada de $e_{\max }=0.034 \mathrm{~m}$. A Figura 46 apresenta a curva Dubins suavizada (Subfig. 46a) e ilustra os componentes de interesse para o Planejador de Trajetórias (Subfigs. 46b e 46c).

Uma segunda curva planejada ilustrada na Figura 47, tem como posturas inicial e final do robô $\boldsymbol{\xi}_{0}=\left[\begin{array}{lll}0 & 0 & \pi / 2\end{array}\right]^{T}$ e $\boldsymbol{\xi}_{F}=\left[\begin{array}{lll}4 & 1 & 2 \pi / 3\end{array}\right]^{T}$, e com um raio máximo de curvatura $\rho=1 \mathrm{~m}$. O algoritmo de Walker para estes parâmetros retorna uma geodésica do tipo $R S L$ com $6,40 \mathrm{~m}$ de comprimento. A suavização com $n+1=9$ waypoints possui um erro máximo de $e_{\max }=0.032 \mathrm{~m}$ entre a geodésica original e a spline, ilustrada na Subfigura 47a. As derivadas dos componentes na configuração alternativa são ilustradas nas Subfiguras 47b e 47c. 


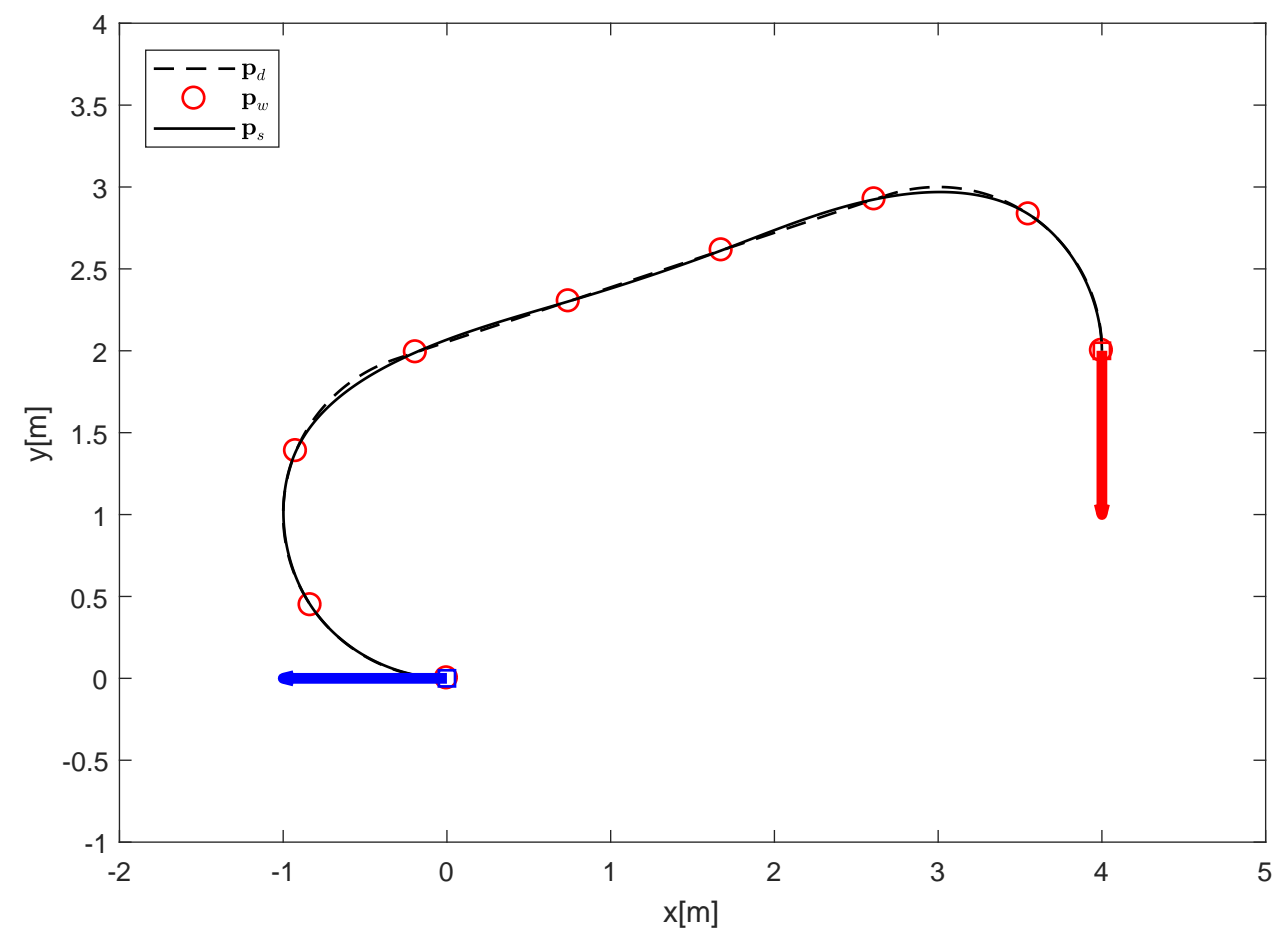

(a)
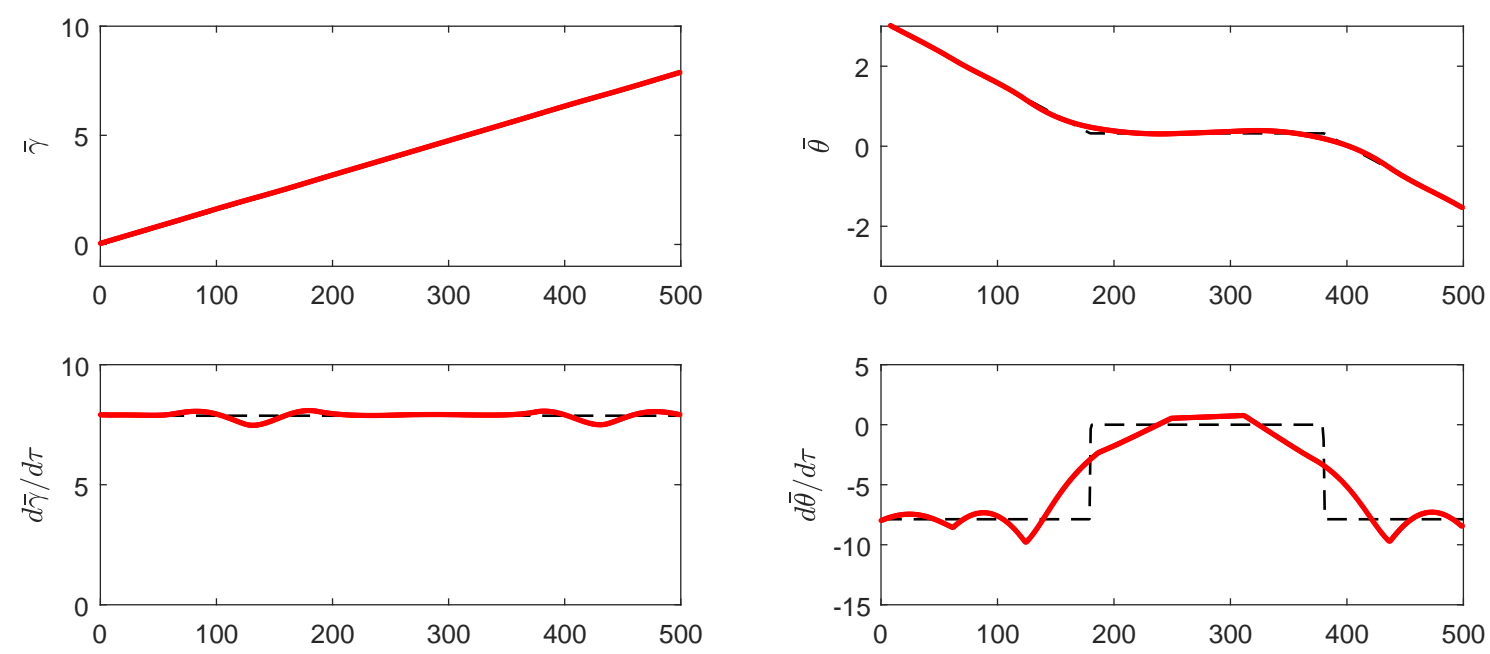

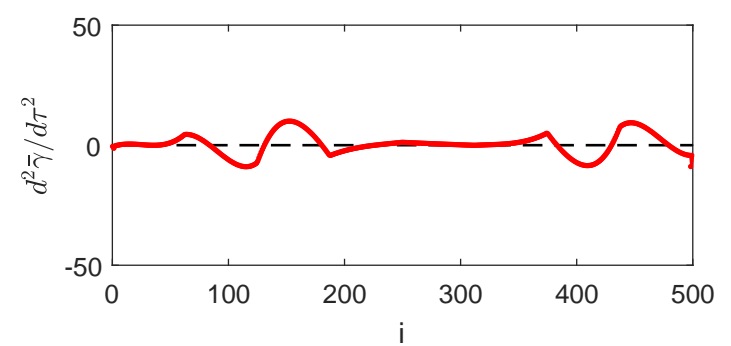

(b)

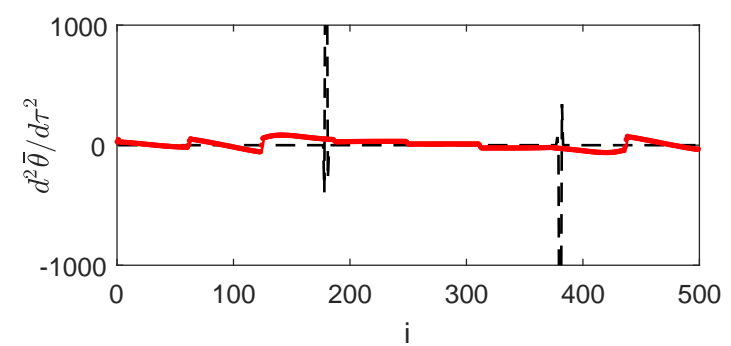

(c)

Figura 46 - Curva Dubins suavizada tipo $R S R$ e suas componentes de $\overline{\mathbf{s}}_{i}$ Fonte: o autor. 


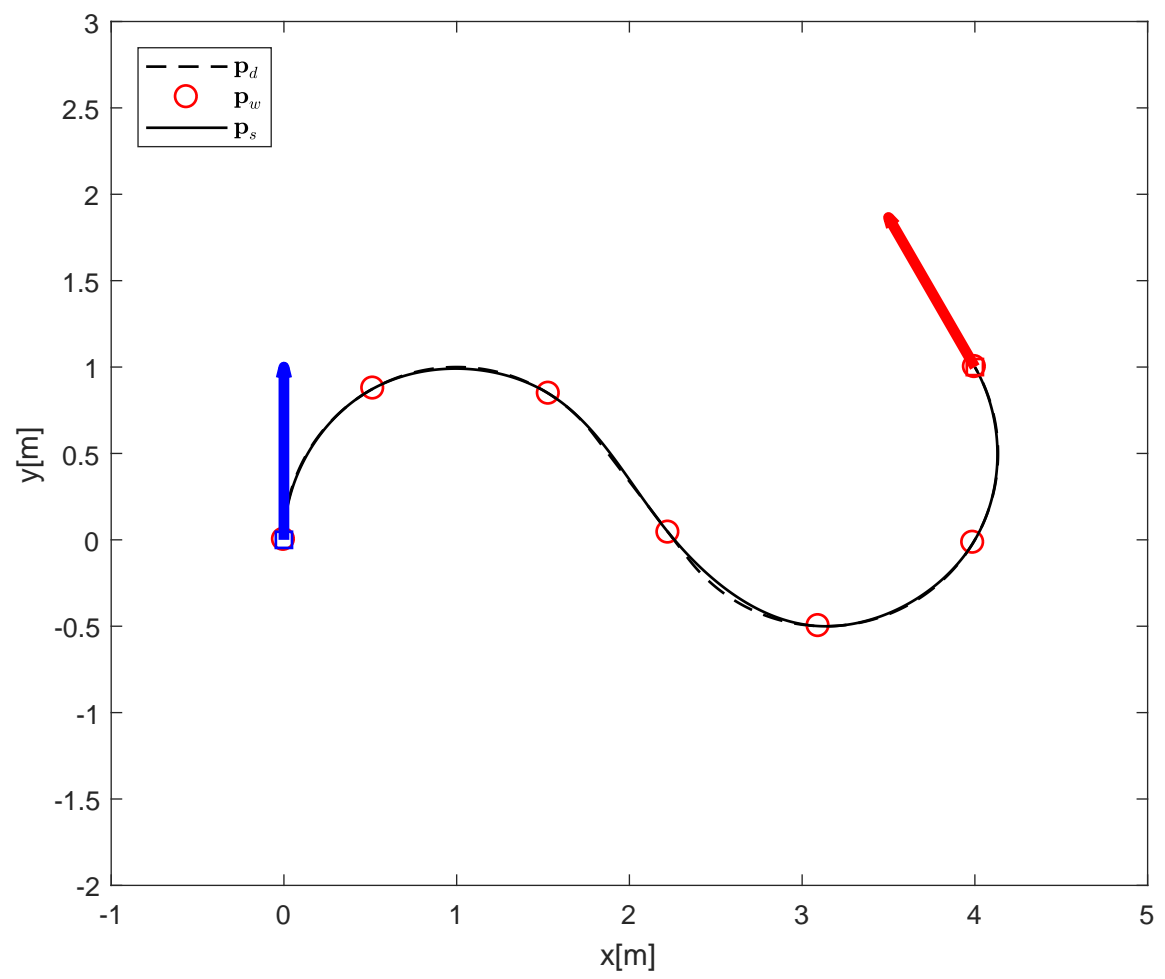

(a)
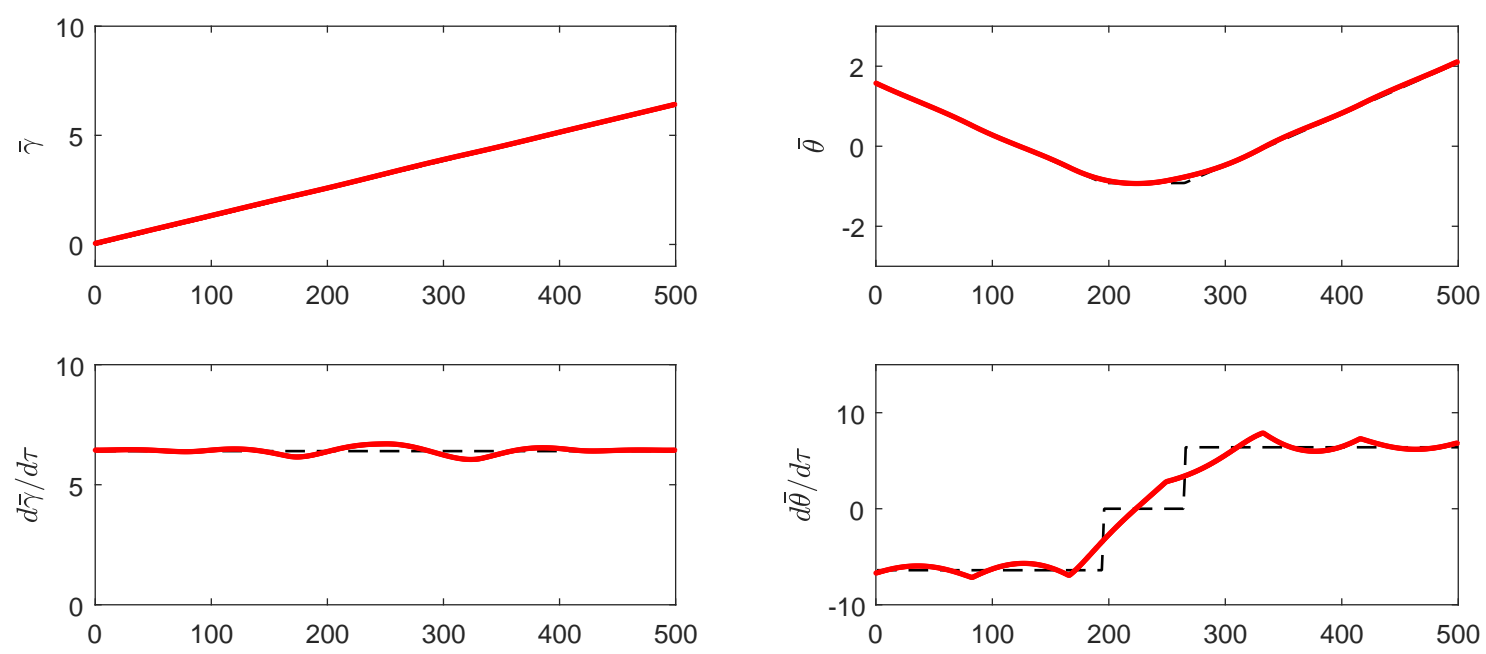

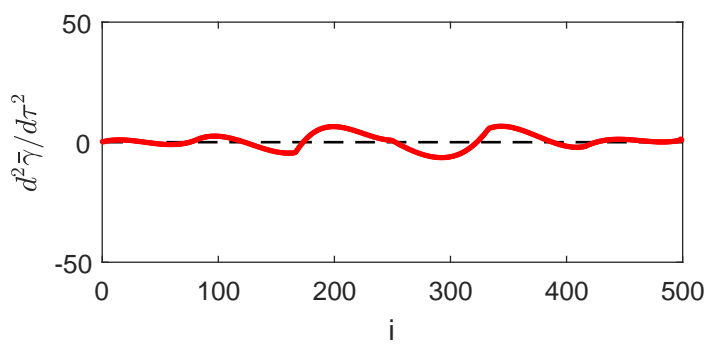

(b)

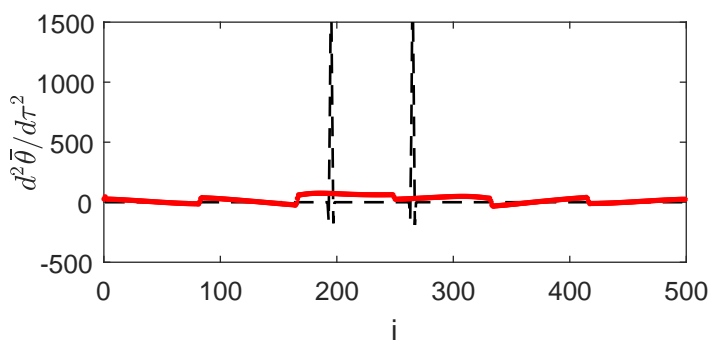

(c)

Figura 47 - Curva Dubins suavizada tipo $R S L$ e suas componentes de $\overline{\mathbf{s}}_{i}$

Fonte: o autor. 


\subsection{CONCLUSÃO DO CAPÍTULO}

A etapa de Planejamento de Caminho entre duas posturas distintas em um ambiente livre de obstáculos encontra na literatura sua grande referência nos trabalhos de Dubins (1957). Dado um raio mínimo, a geodésica de Dubins é o caminho ótimo entre duas posturas e, portanto, mais eficiente em termos energéticos para uma velocidade linear constante.

Entretanto, trajetórias com velocidades arbitrárias dentro do caminho de Dubins irão apresentar diferentes relações entre tempo e energia. O objetivo do Planejador de Trajetória é encontrar, dentre as possíveis trajetórias, aquela que seja ótima, baseada em um parâmetro de ponderação entre tempo e energia.

Porém uma característica específica de curvas Dubins é que não permitem sua utilização de forma direta como caminho a ser percorrido pelo robô, no algoritmo de Planejamento de Trajetória proposto nessa Tese - por serem compostas de arcos de circunferência (que variam o ângulo de orientação) e segmentos de reta (que mantêm a orientação do robô); na transição entre estas partes, a derivada de segunda ordem dos componentes de seu vetor de configuração alternativa é descontínua. Esta característica impossibilita algoritmos de otimização tratarem o problema, pois o sistema de restrições é uma matriz mal-condicionada.

Para resolver este problema, o Capítulo propôs uma suavização do caminho mínimo de Dubins sem descaracterizar sua curva e as orientações inicial e final do robô. Para tanto, uma série de waypoints a cada aproximadamente $1 \mathrm{~m}$, foi posicionado no caminho de Dubins. Uma curva interpolante utilizando spline cúbica grampeada passando por estes waypoints é então proposta como caminho alternativo ao robô. Como estas curvas por partes são compostas por polinômios cúbicos com continuidade $C^{2}$ em toda a sua extensão, garante-se que a matriz de restrições dinâmicas do problema de otimização de trajetória seja condicionada, a tal ponto que o algoritmo de otimização possa convergir para uma trajetória ótima. Um código simplificado desta suavização em MatLab ${ }^{\circledR}$ é apresentado no Apêndice B.3. 


\section{RASTREAMENTO DE TRAJETÓRIA}

Uma proposta de planejamento de caminho baseado nas cuvas de Dubins foi apresentada no Capítulo 4. Anteriormente, no Capítulo 3, a trajetória do robô em um caminho fixo foi planejada levando-se em consideração um compromisso entre tempo e energia. Neste Capítulo, propõe-se um controlador para que o robô possa efetivamente realizar esta trajetória.

\subsection{DEFINIÇÃO DO PROBLEMA}

O caminho geométrico $\Gamma$ pelo qual o robô deve percorrer é definido por um conjunto de $N+1$ pontos definidos por $\mathbf{p}_{c_{i}}=\left[\begin{array}{ll}x_{c_{i}} & y_{c_{i}}\end{array}\right]^{T}, i=0, \ldots, N$. A trajetória, por sua vez, é definida pelo conjunto do caminho geométrico e pela série associada de tempos

$$
t_{i}=\sum_{j=0}^{i} \delta t_{j}, \quad i=0, \ldots, N
$$

tal que

$$
\delta t_{i}=\frac{\delta \tau_{i}}{\sqrt{b_{i}^{*}}}, \quad i=1, \ldots, N,
$$

onde $b^{*}$ é o parâmetro $b$ ótimo estimado pelo Planejamento de Trajetória, e $\delta \tau_{i}=\tau_{i}-\tau_{(i-1)}$, $\tau_{0}=0, b_{0}=0$. Além disso, o robô deve estar orientado, a cada ponto, na direção do próximo ponto discreto da curva, ou seja,

$$
\theta_{c_{i}}=t g^{-1}\left(\frac{y_{c_{i}+1}-y_{c_{i}}}{x_{c_{i}+1}-x_{c_{i}}}\right), \quad i=0, \ldots, N-1 .
$$

No extremo, é satisfatório considerar $\theta_{c_{N}}=\theta_{c_{(N-1)}}$. Com estas considerações, a postura desejada para o robô é $\boldsymbol{\xi}_{c_{i}}=\left[\begin{array}{lll}x_{c_{i}} & y_{c_{i}} & \theta_{c_{i}}\end{array}\right]^{T}, i=0, \ldots, N$ é associado a um tempo discreto $t_{i}$, onde $i=0, \ldots, N$.

O problema de Rastreamento de Trajetória é encontrar uma ação de controle de tensões nos motores $\mathbf{u}_{i}$, tal que o robô, partindo de uma configuração inicial $\boldsymbol{\xi}_{c_{0}}$, siga a postura desejada $\boldsymbol{\xi}_{c_{i}}$ em cada tempo discreto.

\subsection{CONTROLE EM MALHA ABERTA}

O problema de mínimo tempo e energia otimizado pelo Planejamento de Trajetória tem, entre suas variáveis de otimização, o sinal de controle $\mathbf{u}_{i}=\left[\begin{array}{ll}u_{r i} & u_{l i}\end{array}\right]$ do robô. Ao final do processo de otimização, o algoritmo fornece o controle ótimo $\mathbf{u}_{i}^{*}=\left[\begin{array}{ll}u_{r i}^{*} & u_{l i}^{*}\end{array}\right]$, tal que o modelo dinâmico do robô lhe conduza a seguir o caminho pré-definido. 
Seja então a curva $\Gamma$ utilizada como exemplo no Capítulo 3, discretizada em $N=500$ pontos. O processo de linearização discreta apresentada na seção 3.3 é então realizada na curva.

A curva $\Gamma$, seus componentes em coordenadas alternativas no ponto médio de $\overline{\mathbf{s}}_{c_{i}}=\left[\begin{array}{ll}\gamma_{c_{i}} & \theta_{c_{i}}\end{array}\right]^{T}$ e as derivadas de primeira e segunda ordem, $\overline{\mathbf{s}}_{c_{i}}^{\prime}$ e $\overline{\mathbf{s}}_{c_{i}}^{\prime \prime}$, respectivamente, são apresentadas na Figura 48.

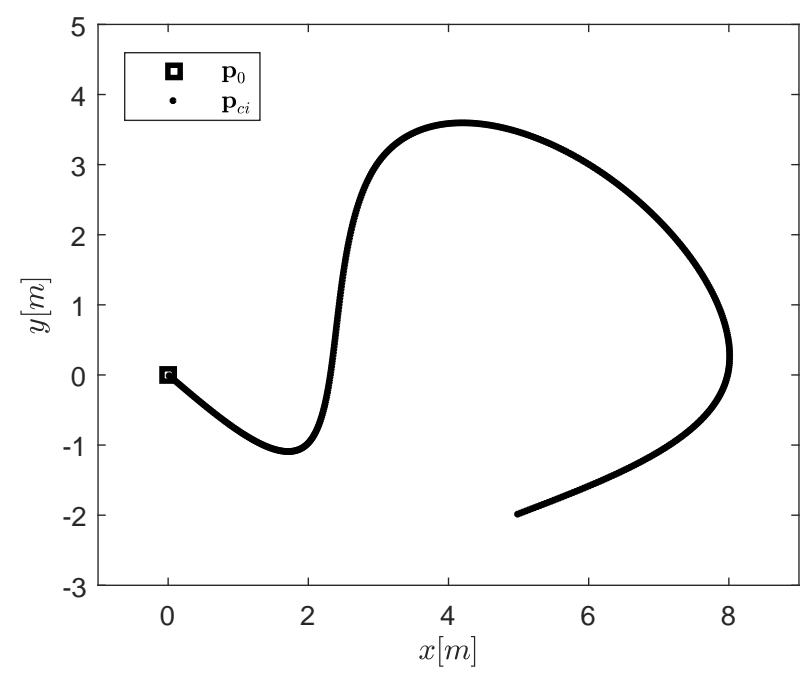

(a) Curva $\Gamma$ no plano cartesiano. O quadrado representa o ponto inicial $\mathbf{p}_{0}$.
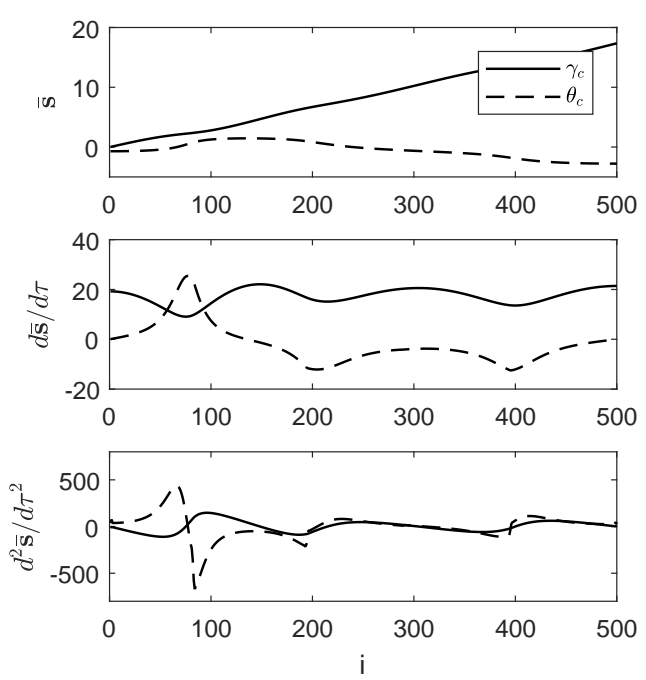

(b) Componentes da curva $\Gamma$ no tempo médio discreto $\overline{\mathbf{s}}_{c_{i}}$, e suas derivadas de primeira e segunda ordem.

Figura 48 - Curva $\Gamma$ e suas derivadas para a otimização, $N=500$.

Fonte: o autor.

O Problema 3.4 de otimização tempo-energia é resolvido com os dados da curva $\Gamma$, para um coeficiente de ponderação arbitrariamente sintonizado com o valor do coeficiente de ponderação $\mu=100$. O resultado do algoritmo de otimização Mosek para as tensões dos motores é apresentado na Figura 49.

Então, o modelo dinâmico do robô (eq. 3.15) é discretizado da segunte forma

$$
\begin{aligned}
\ddot{\gamma}_{i} & =\frac{K_{m}}{m r}\left(u_{r i}+u_{l i}\right), \\
\ddot{\theta}_{i} & =\frac{K_{m} B}{J 2 r}\left(u_{r i}-u_{l i}\right),
\end{aligned}
$$

e os componentes da configuração alternativa do robô são calculados a cada $i$ pelos somatórios

$$
\begin{aligned}
& \gamma_{i}=\sum_{k=0}^{i}\left(\sum_{j=0}^{i} \ddot{\gamma}_{j} \delta t_{j}\right) \delta t_{k}, \\
& \theta_{i}=\sum_{k=0}^{i}\left(\sum_{j=0}^{i} \ddot{\theta}_{j} \delta t_{j}\right) \delta t_{k} .
\end{aligned}
$$



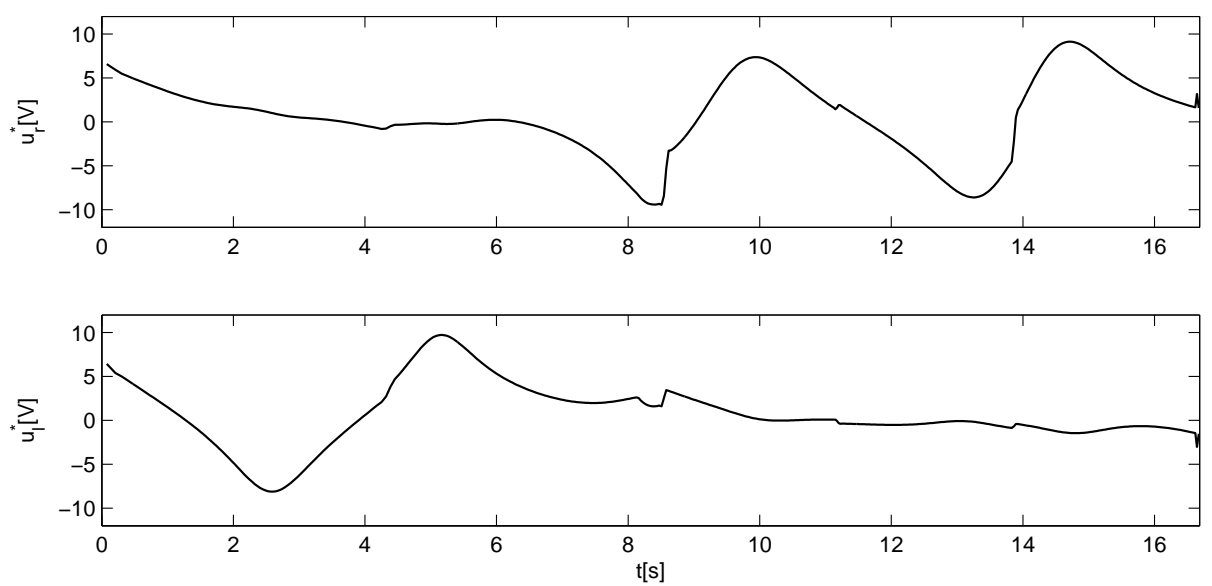

Figura 49 - Gráficos de valores de tensão ótima para a curva $\Gamma$, com $\mu=100$. No gráfico superior, apresenta-se a tensão ótima do motor direito $u_{r}^{*}$ e no gráfico inferior, a tensão ótima do motor esquerdo $u_{l}^{*}$.

Fonte: o autor.

Os sinais de avanço e de orientação da configuração alternativa podem ser transformados nos sinais de posição $x_{i}$ e $y_{i}$ pelas Equações 3.12. Usando o sinal ótimo do planejamento como sinal de controle para o robô, $\mathbf{u}_{i}=\mathbf{u}_{i}^{*}=\left[\begin{array}{ll}u_{r i}^{*} & u_{l i}^{*}\end{array}\right]$, o controle em malha aberta discreto do sistema pode ser representado pelo diagrama de blocos da Figura 50.

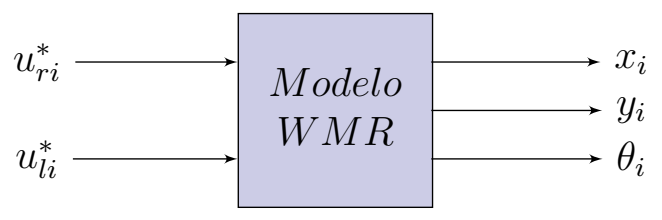

Figura 50 - Controle em malha aberta. Fonte: o autor.

Para o sinal de controle ótimo dado, a resposta do modelo do robô é a série $\mathbf{p}_{M A_{i}}=\left[\begin{array}{ll}x_{i} & y_{i}\end{array}\right]^{T}$, ilustrada na Figura 51 juntamente com a curva de referência $\Gamma$.

Definem-se então os erros de rastreamento de avanço $e_{\gamma_{i}}$ e o erro de rastreamento de orientação $e_{\theta_{i}}$ como sendo

$$
\begin{aligned}
& e_{\gamma_{i}}=\sqrt{\left(x_{c_{i}}-x_{i}\right)^{2}+\left(y_{c_{i}}-y_{i}\right)^{2}}, \\
& e_{\theta_{i}}=\theta_{c_{i}}-\theta_{i} .
\end{aligned}
$$

Percebe-se na Subfigura 51a a existência de erros de rastreamento, pois a curva de resposta em malha fechada não coincide com a curva de referência. Estes erros ficam evidenciados na Subfigura 51b, onde os erros de rastreamento de avanço e de orientação são grafados em função do tempo. 
O erro de rastreamento de avanço máximo durante o trajeto é de $\max \left\{e_{\gamma}\right\}=$ 0,125m, o que equivale a quase um terço da largura do robô. Em certas condições, este erro pode ser crucial para a eficácia do movimento.

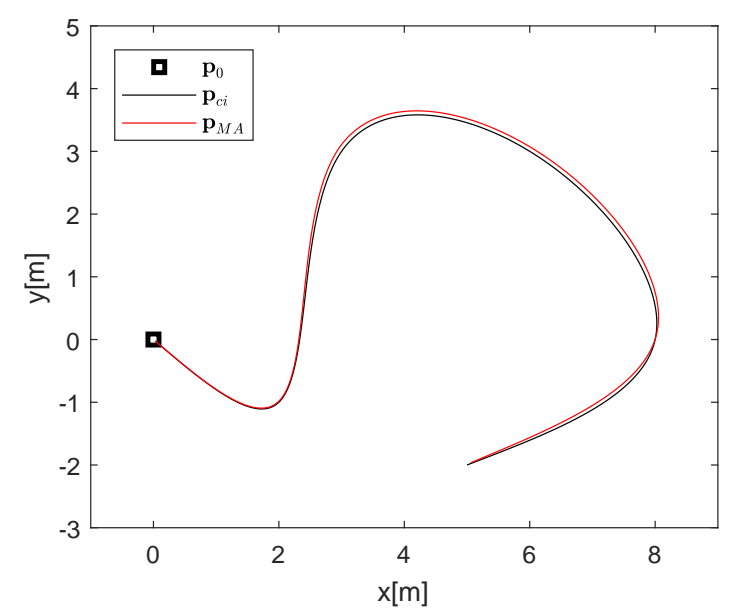

(a) Erro no plano para a curva $\Gamma$
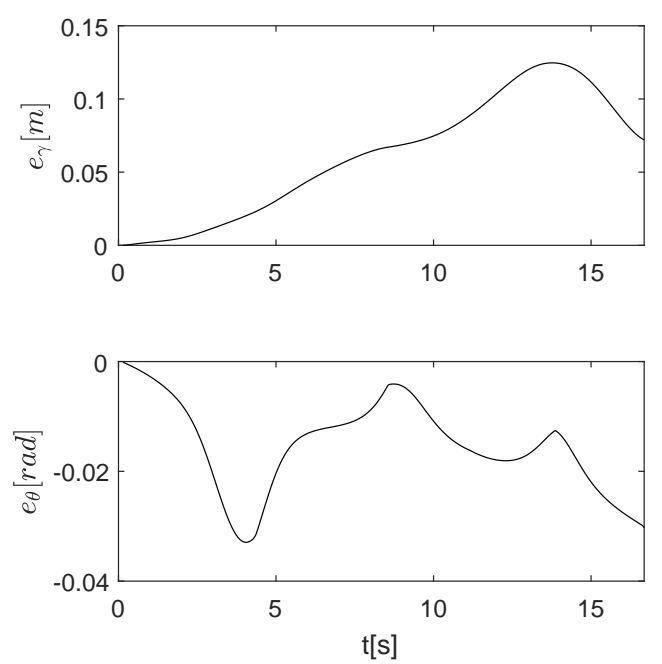

(b) Erros nas coordenadas para a curva $\Gamma$

Figura 51 - Gráficos de resposta em malha aberta para a curva $\Gamma$. À esquerda, a curva no plano; e à direita, erros de avanço e orientação em função do tempo.

Fonte: o autor.

Foram realizados experimentos numéricos em três outras diferentes curvas de referência, como ilustra a Figura 52. As três curvas Dubins suavizadas foram discretizadas utilizando $N=500$ pontos, com trajetória otimizada em tempo e energia com um coeficiente $\mu=100$. Os sinais de controle ótimo calculados pelo algoritmo de otimização são utilizados como entrada em malha aberta do modelo do robô. Os gráficos da esquerda na Figura 52 ilustram as curvas de referência e de saída do modelo, ao passo que os gráficos da direita ilustram os erros de rastreamento.

Percebe-se nos experimentos extras que o controle ótimo calculado pelo Planejador de Trajetória não é eficaz no Rastreamento de Trajetória.

Como trata-se de um sistema discreto, onde as derivadas são estimadas e as integrações são resultado de somatórios em um campo com tempo finito, os erros se acumulam continuamente nestes somatórios. A análise numérica apresentada na subseção 3.5.1 ilustra este efeito.

Propõe-se então apresentar um controlador em malha fechada para o Rastreamento de Trajetória, considerando os erros geométricos de rastreamento, linearizando a cinemática do robô. 


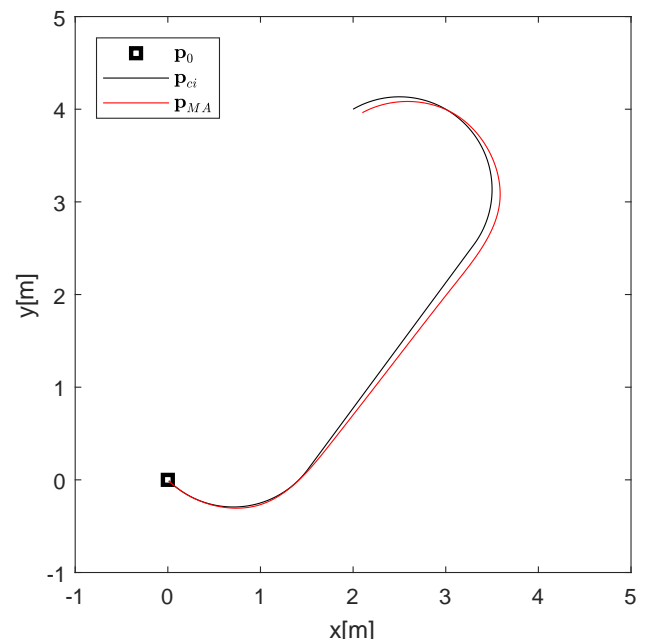

(a) Erro no plano para uma curva Dubins suave $L S L$

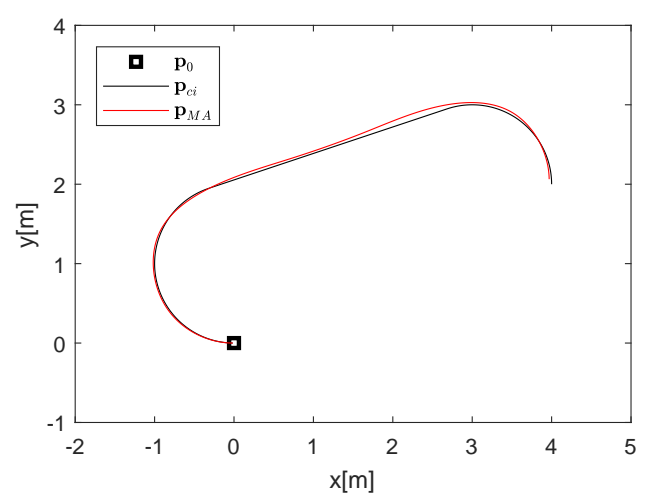

(c) Erro no plano para uma curva Dubins suave $R S R$

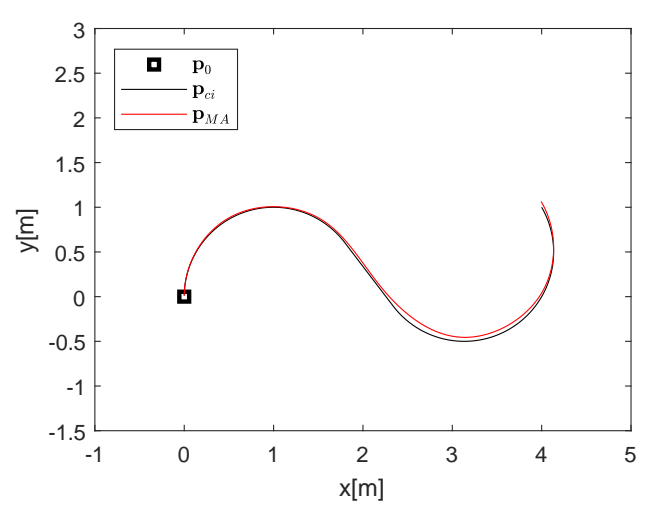

(e) Erro no plano para uma curva Dubins suave $R S L$
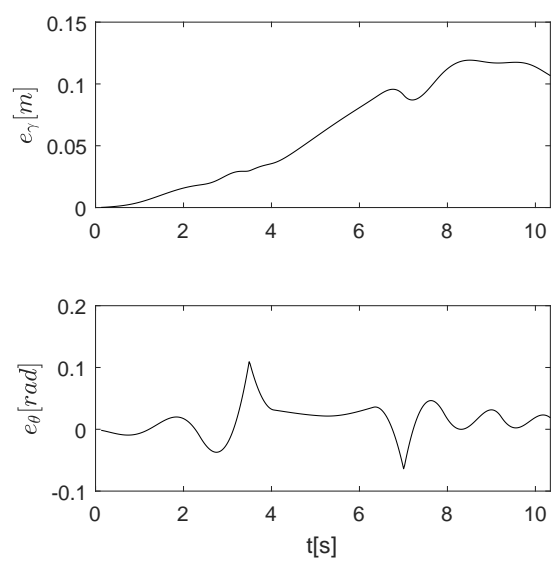

(b) Erros nas coordenadas para uma curva Dubins suave $L S L$
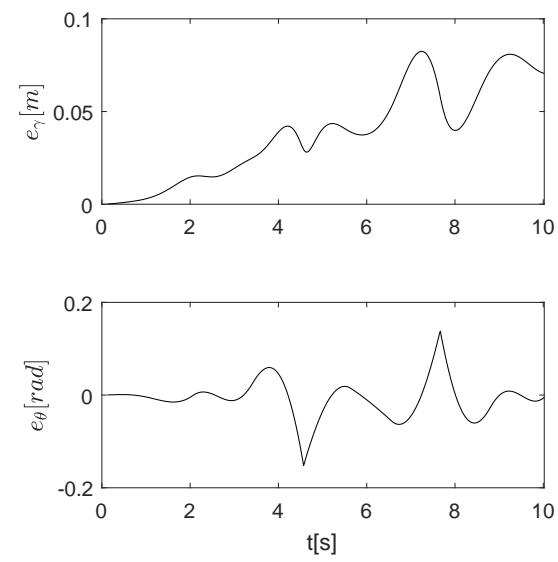

(d) Erros nas coordenadas para uma curva Dubins suave $R S R$
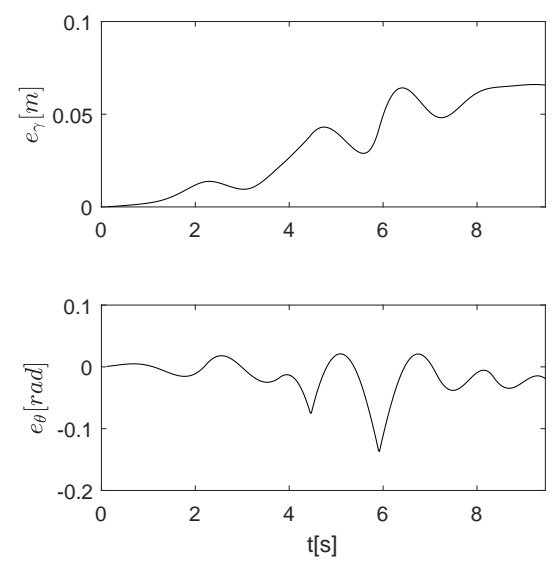

(f) Erros nas coordenadas para uma curva Dubins suave $R S L$

Figura 52 - Gráficos de resposta em malha aberta para alguns tipos de curvas.

Fonte: o autor. 


\subsection{A ABORDAGEM "SIGA A CENOURA"}

A abordagem "Siga a Cenoura" (do inglês follow the carrot) foi apresentada inicialmente por Rankin (1997) como uma solução para o problema de seguimento de caminho. Esta abordagem deriva da ideia de segurar uma cenoura à frente de um cavalo, induzindo o animal a se movimentar naquela direção. Com esta idéia, o método calcula a orientação do veículo, tal que este aponte para um ponto no caminho chamado ponto cenoura.

Seja um ponto de referência $C$, definido pelo vetor de posição $\mathbf{p}_{c}(t)=\left[\begin{array}{ll}x_{c}(t) & y_{c}(t)\end{array}\right]^{T}$, transladando sobre o caminho $\Gamma$ com uma velocidade escalar $v_{c}$ tangencial à curva $\Gamma$. A posição do robô $P$ em função tempo $t$ é definida por $\mathbf{p}(t)=\left[\begin{array}{ll}x(t) & y(t)\end{array}\right]^{T}$, e sua velocidade escalar por $v=\sqrt{\dot{x}^{2}+\dot{y}^{2}}$.

Define-se o erro linear $\left(e_{\gamma}\right)$ como a distância geométrica entre os pontos de referência $C$ e a posição do robô $P$, menos um hiato geométrico $\varrho$, dado por

$$
e_{\gamma}=\sqrt{e_{x}^{2}+e_{y}^{2}}-\varrho
$$

onde $e_{x}=\left(x_{c}-x\right)$ e $e_{y}=\left(y_{c}-y\right)$. Define-se também o erro de orientação como sendo a diferença angular entre o segmento de reta que liga os pontos $C$ e $P$ e a orientação do robô $\theta$, dado por

$$
e_{\theta}=\tan ^{-1}\left(\frac{e_{y}}{e_{x}}\right)-\theta
$$

O hiato geométrico $\varrho$ se faz necessário pois se os pontos $C$ e $P$ coincidirem, tem-se $e_{x}=e_{y}=0$, trazendo uma singularidade para a Equação 5.11. Este modelo de problema é ilustrado na Figura 53.

O objetivo do controle é minimizar os erros $e_{\gamma}$ e $e_{\theta}$ de tal forma que o robô siga o ponto de referência a uma distância $\varrho$.

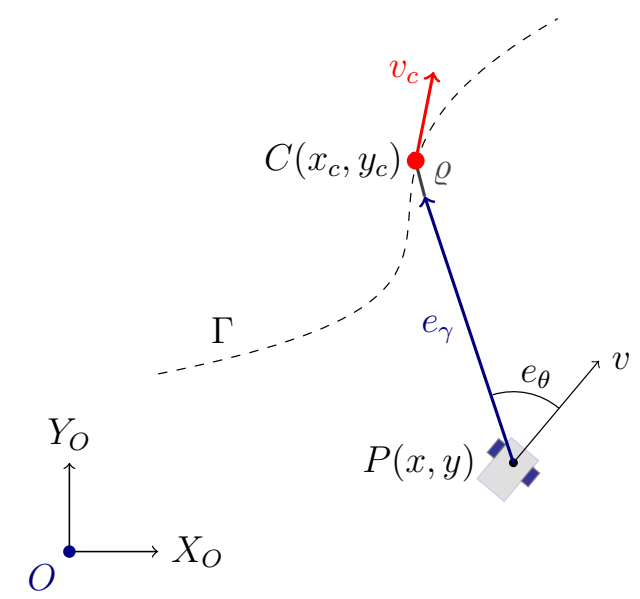

Figura 53 - Modelo de rastreamento de trajetória.

Fonte: o autor. 
Usualmente se utilizam estratégias de controle linear, como controlador Proporcional Integral -PI (BARTON, 2001), e controlador Proporcional Integral Derivativo - PID (WIT, 2000; HOGG et al., 2002), as quais foram usadas de forma satisfatória para a resolução do problema de seguimento de caminho (WIT; CRANE; ARMSTRONG, 2004; HELLSTROM; RINGDAHL, 2006; DUAN et al., 2013; SUJIT; SARIPALLI; SOUSA, 2013).

\subsection{CONTROLE DE DUPLA MALHA}

\subsubsection{Controle da Cinemática por Feedback Linearization}

Resgatando a cinemática do robô da Equação 2.4, tem-se

$$
\begin{aligned}
& \dot{x}(t)=v(t) \cos \theta(t) \\
& \dot{y}(t)=v(t) \operatorname{sen} \theta(t) \\
& \dot{\theta}(t)=\omega(t) .
\end{aligned}
$$

Derivando no tempo o erro linear (eq. 5.10) e o erro de orientação (eq. 5.11), tem-se:

$$
\begin{aligned}
& \dot{e}_{\gamma}=\frac{e_{x}\left(\dot{x}_{c}-\dot{x}\right)+e_{y}\left(\dot{y}_{c}-\dot{y}\right)}{e_{\gamma}} \\
& \dot{e}_{\theta}=\frac{e_{x}\left(\dot{y}_{c}-\dot{y}\right)-e_{y}\left(\dot{x}_{c}-\dot{x}\right)}{e_{\gamma}^{2}}-\dot{\theta} .
\end{aligned}
$$

Substituindo as equações da cinemática (5.12) nas equações das derivadas dos erros (5.13), após algumas manipulações algébricas tem-se a dinâmica dos erros na forma matricial

$$
\dot{\mathbf{e}}=\mathbf{F}_{1} \dot{\mathbf{p}}_{c}+\mathbf{F}_{2} \mathbf{v}
$$

onde $\dot{\mathbf{e}}=\left[\begin{array}{ll}\dot{e}_{\gamma} & \dot{e}_{\theta}\end{array}\right]^{T}$ é o vetor das derivadas dos erros, $\mathbf{v}=\left[\begin{array}{ll}v & \omega\end{array}\right]^{T}$ é o vetor de entrada do sistema, e as funções $\mathbf{F}_{1}$ e $\mathbf{F}_{2}$, dadas por

$$
\mathbf{F}_{1}=\left[\begin{array}{cc}
+\left(\frac{e_{x}}{e_{\gamma}}\right) & +\left(\frac{e_{y}}{e_{\gamma}}\right) \\
-\left(\frac{e_{y}}{e_{\gamma}^{2}}\right) & +\left(\frac{e_{x}}{e_{\gamma}^{2}}\right)
\end{array}\right], \quad \mathbf{F}_{2}=\left[\begin{array}{cc}
-\frac{\left(e_{x} \cos \theta+e_{y} \operatorname{sen} \theta\right)}{e_{\gamma}} & 0 \\
-\frac{\left(e_{x} \operatorname{sen} \theta-e_{y} \cos \theta\right)}{e_{\gamma}^{2}} & -1
\end{array}\right],
$$

são funções não-lineares dos componentes vetoriais da posição do ponto de referência $\mathbf{p}_{c}=\left[\begin{array}{ll}x_{c} & y_{c}\end{array}\right]^{T}$ e da postura do robô $\boldsymbol{\xi}=\left[\begin{array}{lll}x & y & \theta\end{array}\right]^{T}$. Como mostrado na subseção 2.2.1, esta cinemática só é controlável para trajetórias com velocidades lineares e angulares constantes para a referência. Além disso, a dinâmica do erro é variante no tempo.

Propõe-se então realizar uma linearização por realimentação (feedback linearization $-F B L)$. A idéia central desta metodologia de linearização é transformar algebricamente uma dinâmica não-linear de um sistema em uma dinâmica linear, sob a qual técnicas de controle lineares podem ser aplicadas (ISIDORI, 1995; KHALIL, 1996). 
Seja um saída de controle dada por:

$$
\mathbf{v}_{r}=\mathbf{F}_{2}^{-1} \cdot\left(\mathbf{K e}-\mathbf{F}_{1} \dot{\mathbf{p}}_{c}\right)
$$

onde $\mathbf{v}_{r}=\left[\begin{array}{ll}v_{r} & \omega_{r}\end{array}\right]^{T}, \mathbf{e}=\left[\begin{array}{ll}e_{\gamma} & e_{\theta}\end{array}\right]^{T}$ e $\mathbf{K}=\left(\begin{array}{cc}K_{\gamma} & 0 \\ 0 & K_{\theta}\end{array}\right)$ é uma matriz diagonal de ganhos onde $K_{\gamma}$ é ganho do controlador na dimensão do erro linear, e $K_{\theta}$ é o ganho na dimensão de orientação. Substituindo a saída de controle $\mathbf{v}_{r}$ (eq. 5.16) na entrada do sistema (eq. 5.14), as não-linearidades se cancelam da forma

$$
\begin{aligned}
\dot{\mathbf{e}} & =\mathbf{F}_{1} \dot{\mathbf{p}}_{c}+\mathbf{F}_{2}\left[\mathbf{F}_{2}^{-1} \cdot\left(\mathbf{K e}-\mathbf{F}_{1} \dot{\mathbf{p}}_{c}\right)\right], \\
\dot{\mathbf{e}} & =\mathbf{K e}
\end{aligned}
$$

A dinâmica dos erros è na Equação 5.17 é uma dinâmica proporcional de primeira ordem. Os erros $e_{\gamma}$ and $e_{\theta}$ convergem para zero se e somente se os autovalores de $\mathbf{K}$ são negativos.

\subsubsection{Controle da Dinâmica}

A saída $\mathbf{v}_{r}=\left[\begin{array}{ll}v_{r} & \omega_{r}\end{array}\right]^{T}$ do controlador da cinemática (eq. 5.16), que é função da posição do ponto de referência $\mathbf{p}_{c}(t)=\left[\begin{array}{ll}x_{c}(t) & y_{c}(t)\end{array}\right]^{T}$ e da postura do robô $\boldsymbol{\xi}=\left[\begin{array}{lll}x & y & \theta\end{array}\right]^{T}$, é a velocidade na qual o robô deve ter para que este possa convergir para o ponto de referência - isto é, os erros linear e de orientação convergirem a zero. Porém, a ação efetiva de controle do robô são as tensões nos motores $\mathbf{u}=\left[\begin{array}{ll}u_{r} & u_{l}\end{array}\right]^{T}$.

Resgatando o modelo dinâmico do robô (Equações 3.1 e 3.2), tem-se:

$$
\begin{aligned}
& \dot{v}=\frac{K_{m}}{r}\left(u_{r}+u_{l}\right), \\
& \dot{\omega}=\frac{K_{m} B}{2 r}\left(u_{r}-u_{l}\right) .
\end{aligned}
$$

O objetivo do controle da dinâmica é fazer com que a velocidade do robô $\mathbf{v}=$ $\left[\begin{array}{ll}v & \omega\end{array}\right]^{T}$ convirja para a velocidade de referência $\mathbf{v}_{r}=\left[\begin{array}{ll}v_{r} & \omega_{r}\end{array}\right]^{T}$, que é o resultado da malha de controle da cinemática.

A cada instante de tempo discreto $\delta \tau_{i}$ define-se o erro de velocidade linear $e_{v_{i}}=$ $v_{r_{i}}-v_{i}$ e o erro de velocidade angular $e_{\omega_{i}}=\omega_{r_{i}}-\omega_{i}$. Então, propõe-se um controlador PID discreto com as diferenciais na forma de velocidade (ÅSTRÖM; WITTENMARK, 1997)

$$
\begin{aligned}
\delta v_{i} & =\left(K_{p v}+K_{i v}+K_{d v}\right) e_{v_{i}}-\left(K_{p v}+2 K_{d v}\right) e_{v_{i-1}}+K_{d v} e_{v_{i-2}}, \\
\delta \omega_{i} & =\left(K_{p \omega}+K_{i \omega}+K_{d \omega}\right) e_{\omega_{i}}-\left(K_{p \omega}+2 K_{d \omega}\right) e_{\omega_{i-1}}+K_{d \omega} e_{\omega_{i-2}},
\end{aligned}
$$

onde as constantes $K_{p v}, K_{i v}, K_{d v}$ são os ganhos proporcional, integral e derivativo do controle de velocidade linear, enquanto as constantes $K_{p \omega}, K_{i \omega}, K_{d \omega}$ são os ganhos proporcional, integral e derivativo do controle de velocidade angular. 
Então, as tensões $\mathbf{u}=\left[\begin{array}{ll}u_{r_{i}} & u_{l_{i}}\end{array}\right]$ a cada instante de tempo discreto $\delta \tau_{i}$, são dadas por

$$
\begin{aligned}
& u_{r_{i}}=u_{r_{i-1}}+\delta v_{i}+\delta \omega_{i} \\
& u_{l_{i}}=u_{l_{i-1}}+\delta v_{i}-\delta \omega_{i} .
\end{aligned}
$$

Os incrementos na tensão em ambos os motores $\delta v_{i}$ ocorrerão para compensar o erro de velocidade linear, ao passo que $\delta \omega_{i}$ irá incrementar a tensão do lado direito e decrementar a tensão do lado esquerdo para compensar o erro de velocidade angular do robô. Visando antecipar a convergência do controle da dinâmica do robô, optou-se por considerar o estado inicial das tensões como sendo os valores das tensões ótimas iniciais resultantes do planejamento de trajetória, ou seja, $u_{r}(0)=u_{r}^{*}(0)$ e $u_{l}(0)=u_{l}^{*}(0)$

\subsubsection{Esquema de Controle}

O diagrama de blocos da Figura 54 apresenta um esquema de controle de dupla malha. O Controlador da Cinemática do robô calcula os erros de rastreamento linear $\left(e_{\gamma}\right)$ e de orientação $\left(e_{\theta}\right)$ baseados no esquema "Siga a Cenoura", levando em consideração uma referência de caminho $\mathbf{p}_{c i}=\left[\begin{array}{ll}x_{c i} & y_{c i}\end{array}\right]^{T}$ que o robô deva seguir, e a postura do robô $\boldsymbol{\xi}_{i}=\left[\begin{array}{lll}x_{i} & y_{i} & \theta_{i}\end{array}\right]^{T}$ em cada tempo discreto.

Então, o Controlador Cinemático lineariza a dinâmica dos erros de rastreamento, entregando como saída velocidades linear e angular da forma $\mathbf{v}_{r i}=\left[\begin{array}{ll}v_{r i} & \omega_{r i}\end{array}\right]^{T}$, tais que os erros convirjam para zero. O vetor $\mathbf{v}_{r i}$ é referência para um controlador PID que controla a dinâmica do sistema através das tensões dos dois motores do robô dados pelos sinais $\mathbf{u}_{i}=\left[\begin{array}{ll}u_{r i} & u_{l i}\end{array}\right]^{T}$.

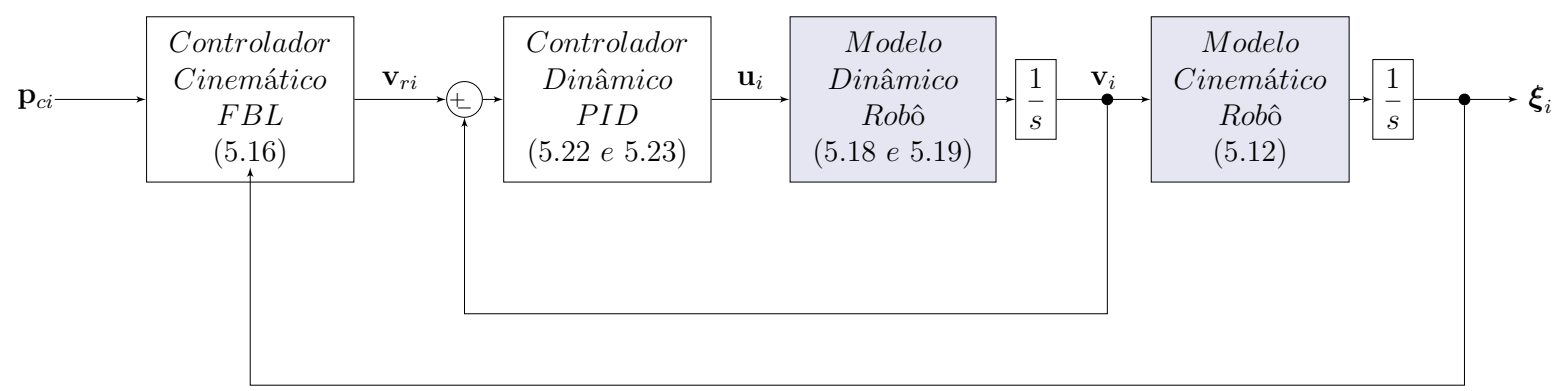

Figura 54 - Diagrama de blocos do controle de dupla malha. A malha interna é relativa ao controle da dinâmica do robô, ao passo que a malha externa realiza o controle da cinemática do robô.

Fonte: o autor.

Para validar o esquema de controle em dupla malha, foram realizadas alguns experimentos numéricos. Seja como primeiro exemplo o caminho descrito pela curva Dubins $L S L$ suavizada, proposta pela Seção 4.3, com referência $\mathbf{p}_{c i}=\left[\begin{array}{ll}x_{c i} & y_{c i}\end{array}\right]^{T}$ a ser seguida pelo robô. 
Os ganhos para o controlador da cinemática do robô são setados em $\mathbf{K}=\left(\begin{array}{cc}-100 & 0 \\ 0 & -10\end{array}\right)$, enquanto os ganhos do controlador PID da dinâmica são setados em $K_{p v}=50 ; K_{i v}=0,1$ e $K_{d v}=10$ para o controlador de velocidade linear, e $K_{p \omega}=10 ; K_{i \omega}=0,5$ e $K_{d \omega}=150$ para o controlador de velocidade angular. Um hiato de $\varrho=0,1 \mathrm{~m}$ foi considerado.

A Figura 55 ilustra o rastreamento no plano cartesiano da curva Dubins $L S L$ suavizada sobre o esquema de controle em dupla malha. Percebe-se que o robô segue de maneira satisfatória a referência dada em todo o seu percurso.

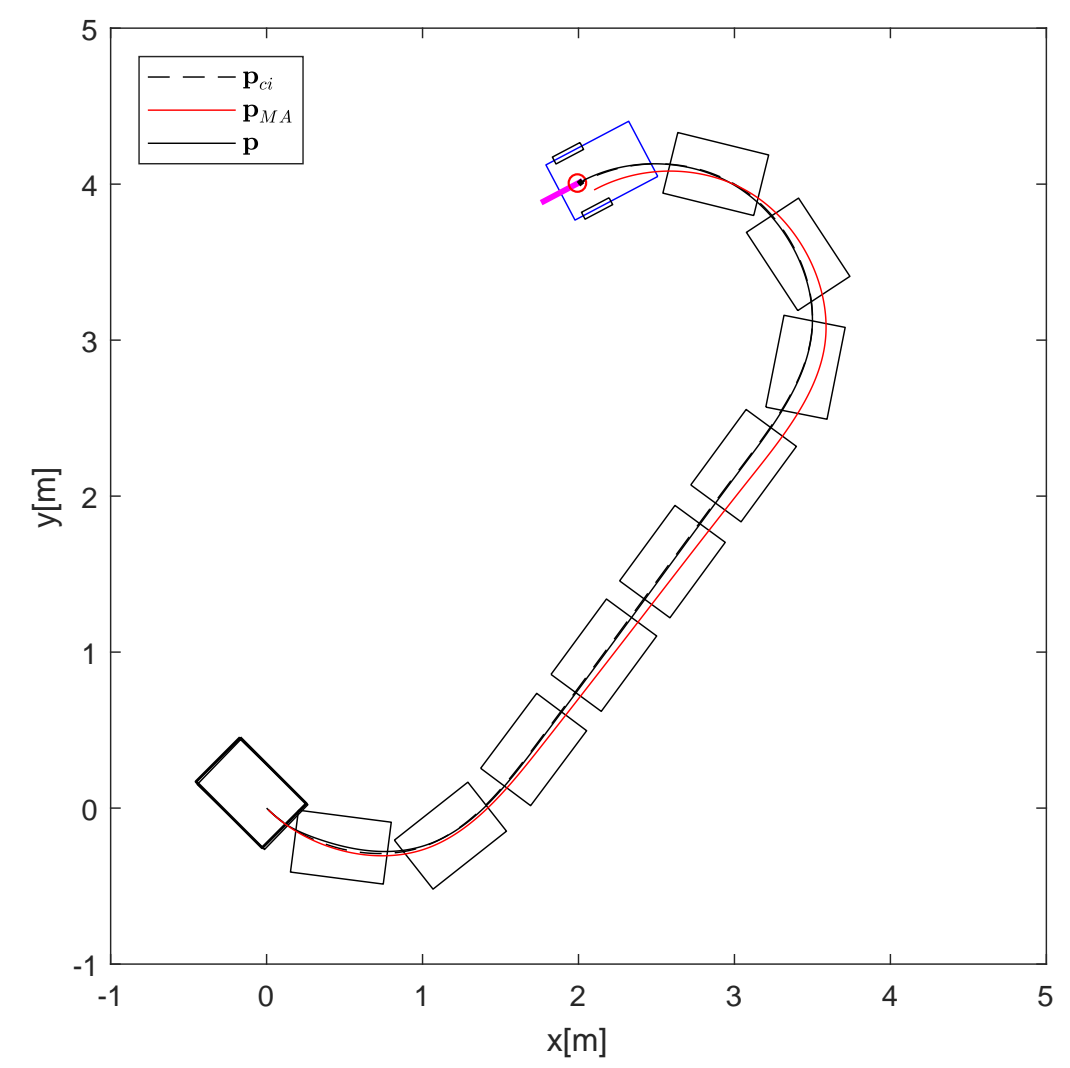

Figura 55 - Rastreamento FBL+PID no plano em uma Dubins Suave tipo LSL. A linha pontilhada representa o caminho de referência, enquanto as linha contínuas ilustram o percurso controlado do robô no plano em malha aberta (em vermelho) e em malha fechada (em preto).

Fonte: o autor.

Para a compreensão da dinâmica do rastreamento neste caminho dado se faz necessário analisar o perfil de velocidade gerado pela etapa de Planejamento de Trajetória. A Figura 56 ilustra o intervalo de integração ótimo gerado pelo algoritmo SOCP dado por $\delta t_{i}=\delta \tau_{i} / \sqrt{b_{i}^{*}}$.

A velocidade inicial do robô no percurso é $v_{0}=0 \mathrm{~m} / \mathrm{s}$. Como os pontos do caminho são praticamente equidistantes (vide Subfigura 44a), para vencer a inércia do robô o intervalo de integração no início do percurso é relativamente alto (inicialmente $\delta t_{0}=0,128 \mathrm{~s}$ ). A partir de aproximadamente $t=1 \mathrm{~s}$, com a inércia inicial do robô vencida, os intervalos de integração diminuem consideravelmente (abaixo de $\delta t_{0}=0,03 \mathrm{~s}$ ). 


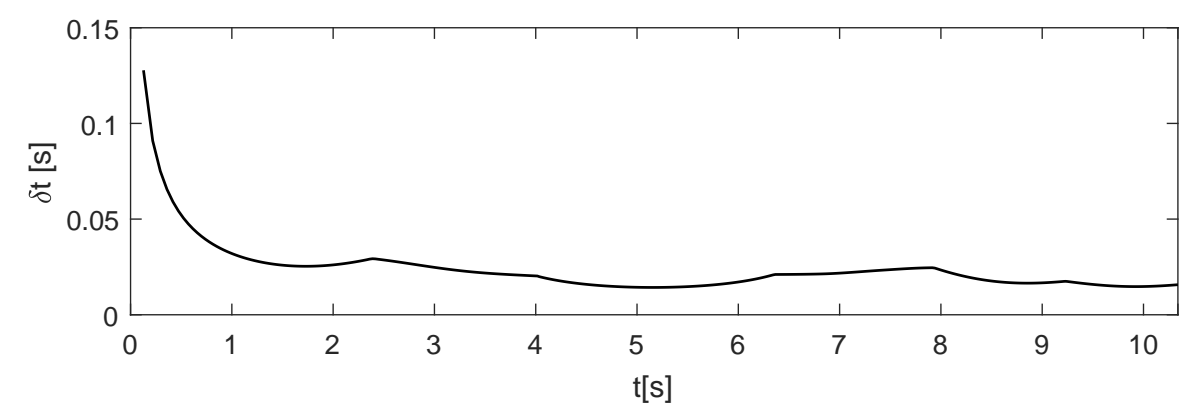

Figura 56 - Intervalo de integração gerada pelo SOCP para a Dubins Suave tipo LSL. Fonte: o autor.

Durante este período transitório inicial, por conta do valor relativamente alto do intervalo de integração, o comportamento das tensões no Controlador da Dinâmica do robô é do tipo bang-bang, limitado pela tensão máxima de $u_{\max }= \pm 12 \mathrm{~V}$. A Figura 57 ilustra os sinais de tensão em ambos os motores durante todo o percurso.
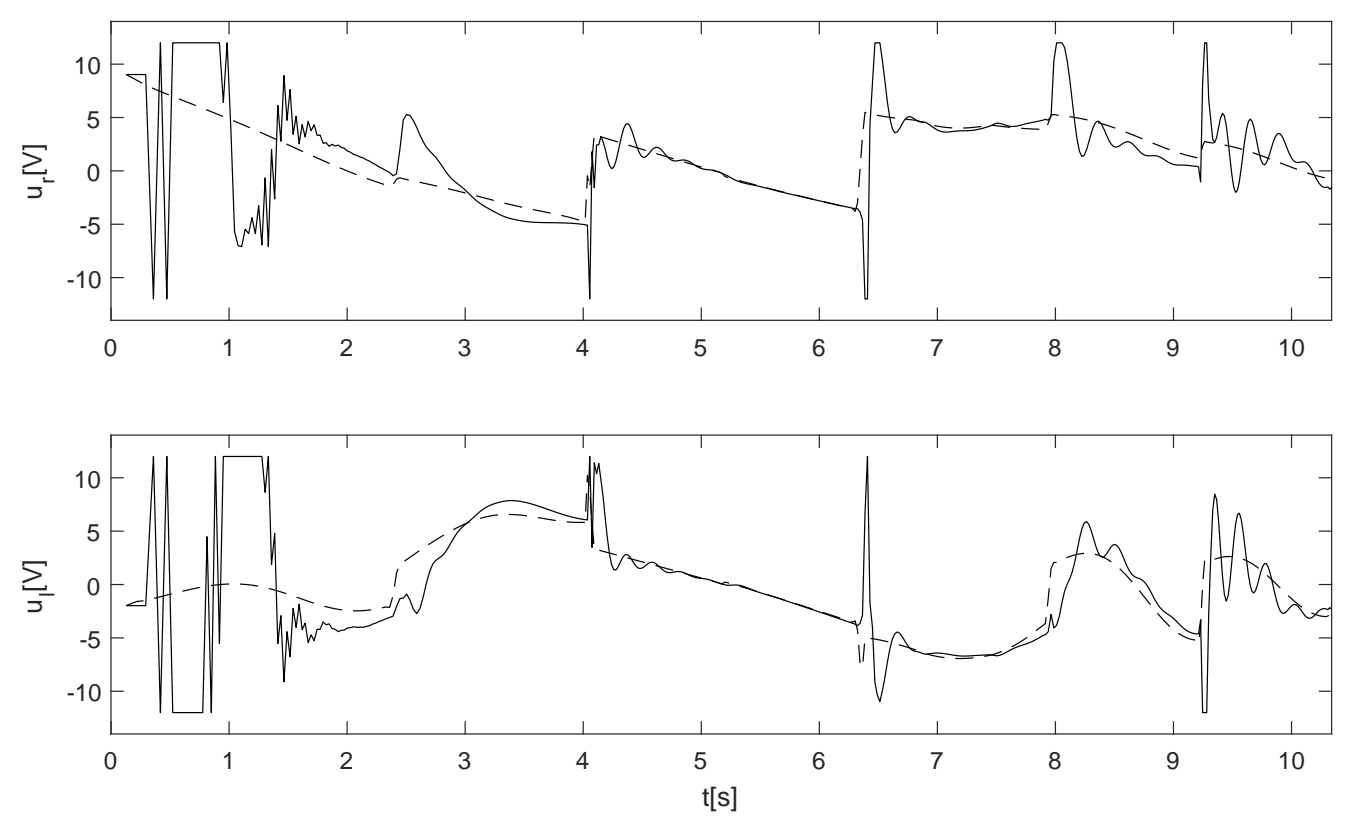

Figura 57 - Tensões de controle para o rastreamento FBL+PID em ambos os motores do robô. Em pontilhado, as tensões ótimas $u^{*}$ do Planejador de Trajetória

Fonte: o autor.

O erro de orientação $e_{\theta}$ neste período transitório também é relativamente elevado, pois a inércia do robô ainda não é vencida, e o trecho inicial do caminho é em curva. Como o erro linear $e_{\gamma}$ ainda é pequeno, o ângulo entre a orientação do robô e o ponto de referência torna-se alto.

Os erros de rastreamento linear $e_{\gamma}$ e de orientação $e_{\theta}$ para o esquema de controle em dupla malha FBL+PID são ilustrados na Figura 58, assim como, a título de comparação, os erros de rastreamento para o esquema de controle em Malha Aberta. 

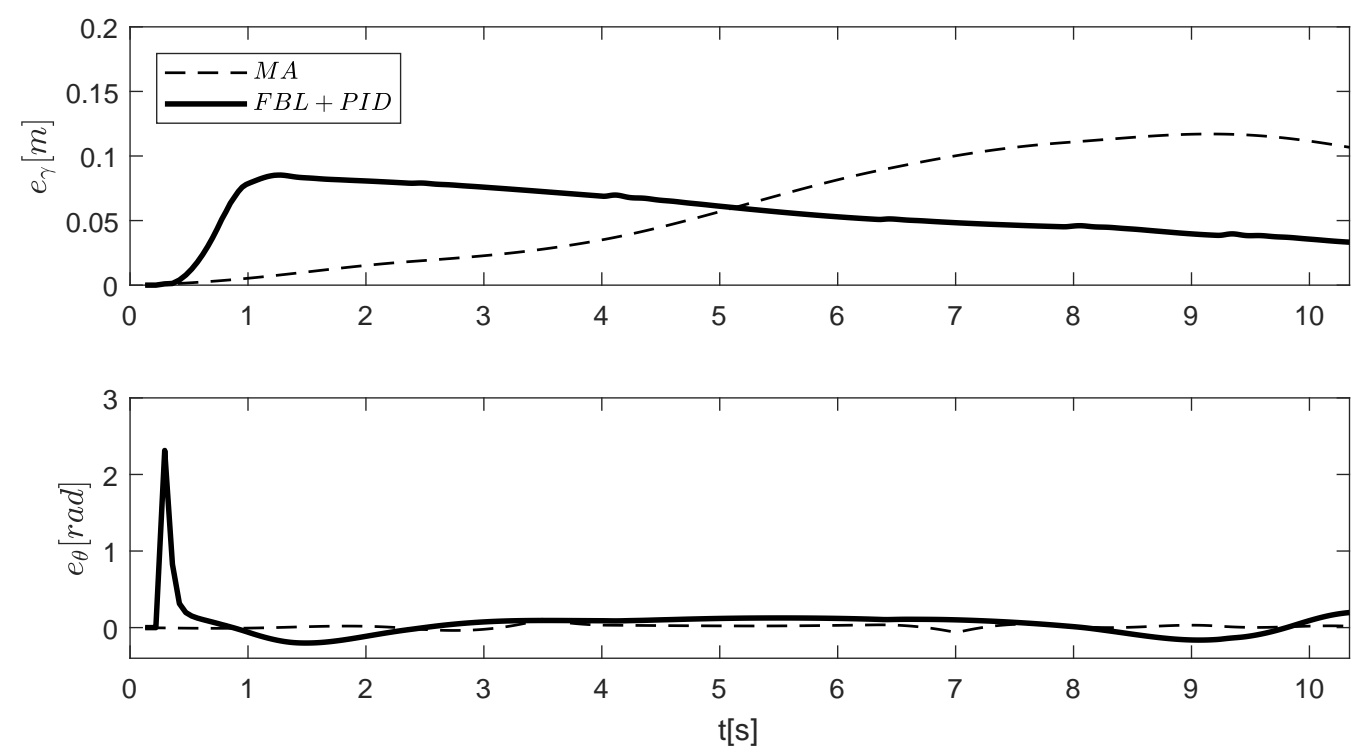

Figura 58 - Erros de rastreamento nas coordenadas alternativas em uma Dubins Suave tipo $L S L$.

Fonte: o autor.

Após este período transitório, quando o intervalo de integração se mantém em um certo regime permanente, o controlador da dinâmica do robô consegue, de maneira satisfatória, rastrear suas referências de velocidade linear e angular, como ilustra a Figura 59, o que se reflete no rastreamento do controlador da cinemática do robô.
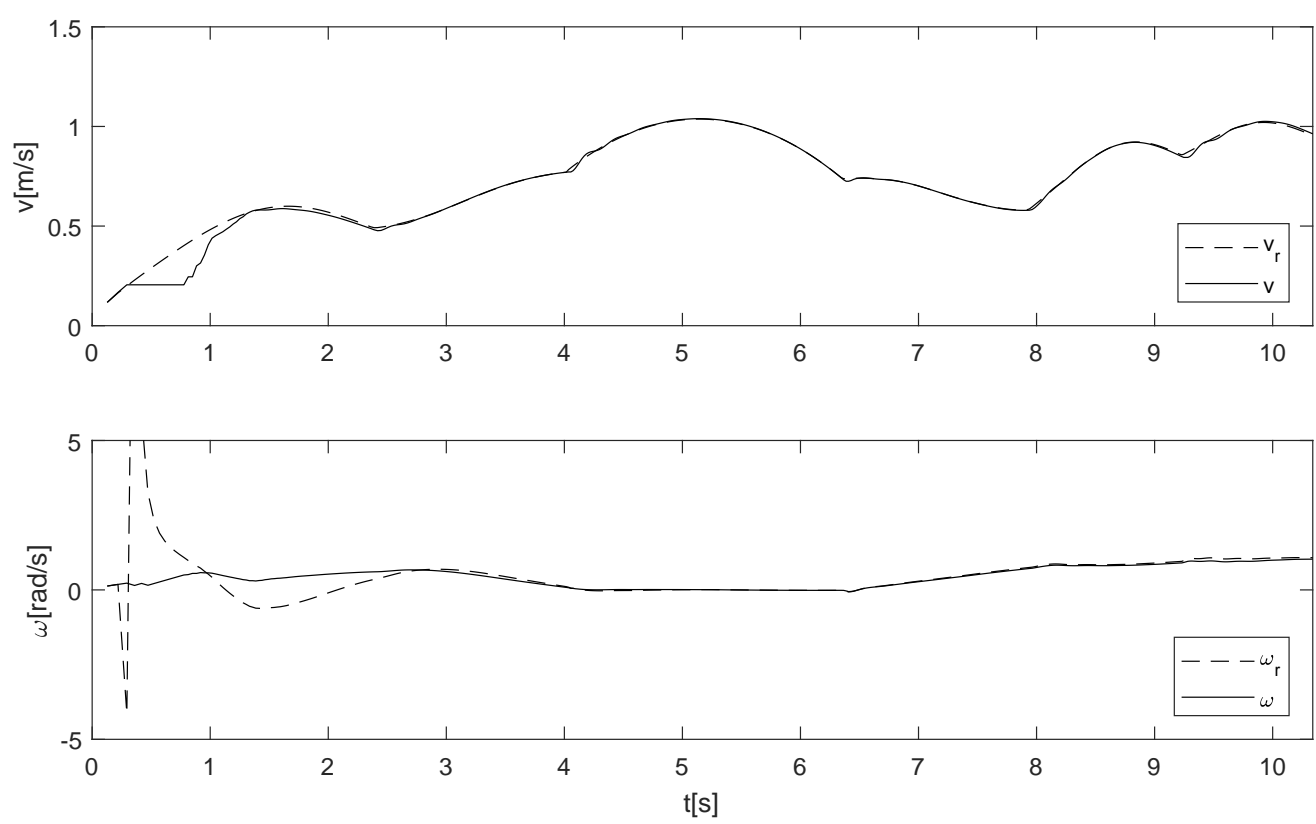

Figura 59 - Velocidades linear e angular para o rastreamento FBL+PID. Em pontilhado, os sinais de referência provenientes do controle da cinemática e em linha contínua as velocidades do robô.

Fonte: o autor. 
Uma segunda simulação numérica foi realizada com um caminho dado por uma curva Dubins Suave tipo $R S R$. As Figuras 60 e 61 ilustram os resultados desta simulação.

Por fim, os resultados da terceira simulação numérica realizada com um caminho dado por uma curva Dubins Suave tipo $R S R$ são ilustrados nas Figuras 62 e 63.

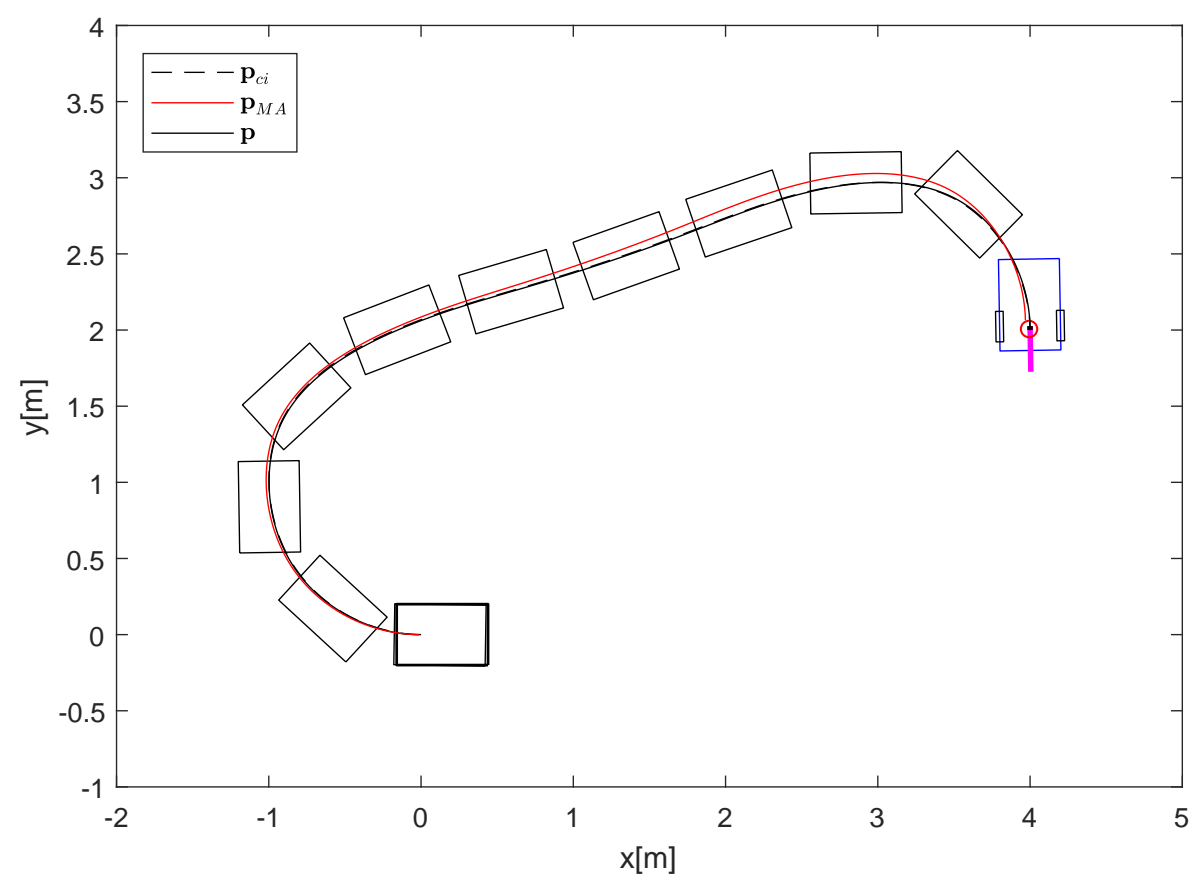

Figura 60 - Rastreamento FBL+PID no plano em uma Dubins Suave tipo $R S R$.

Fonte: o autor.
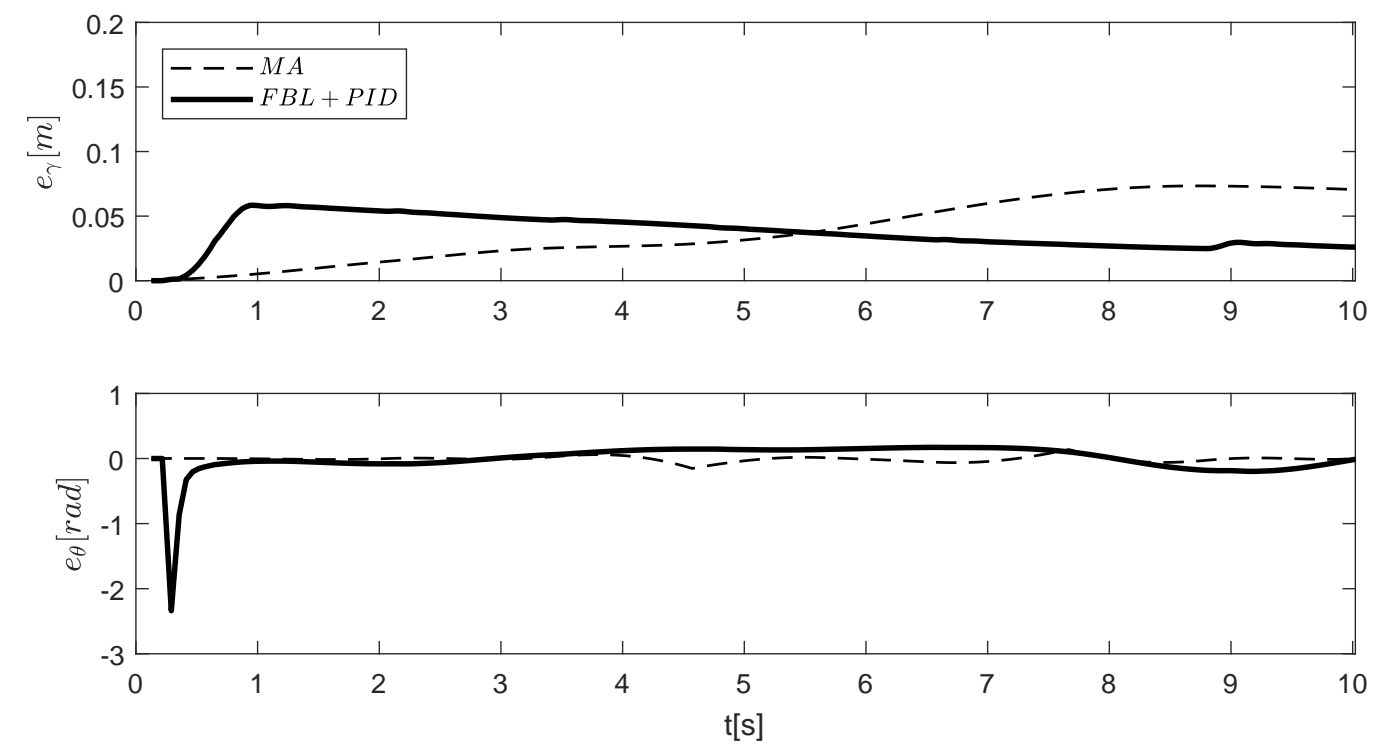

Figura 61 - Erros de rastreamento nas coordenadas alternativas em uma Dubins Suave tipo $R S R$.

Fonte: o autor. 


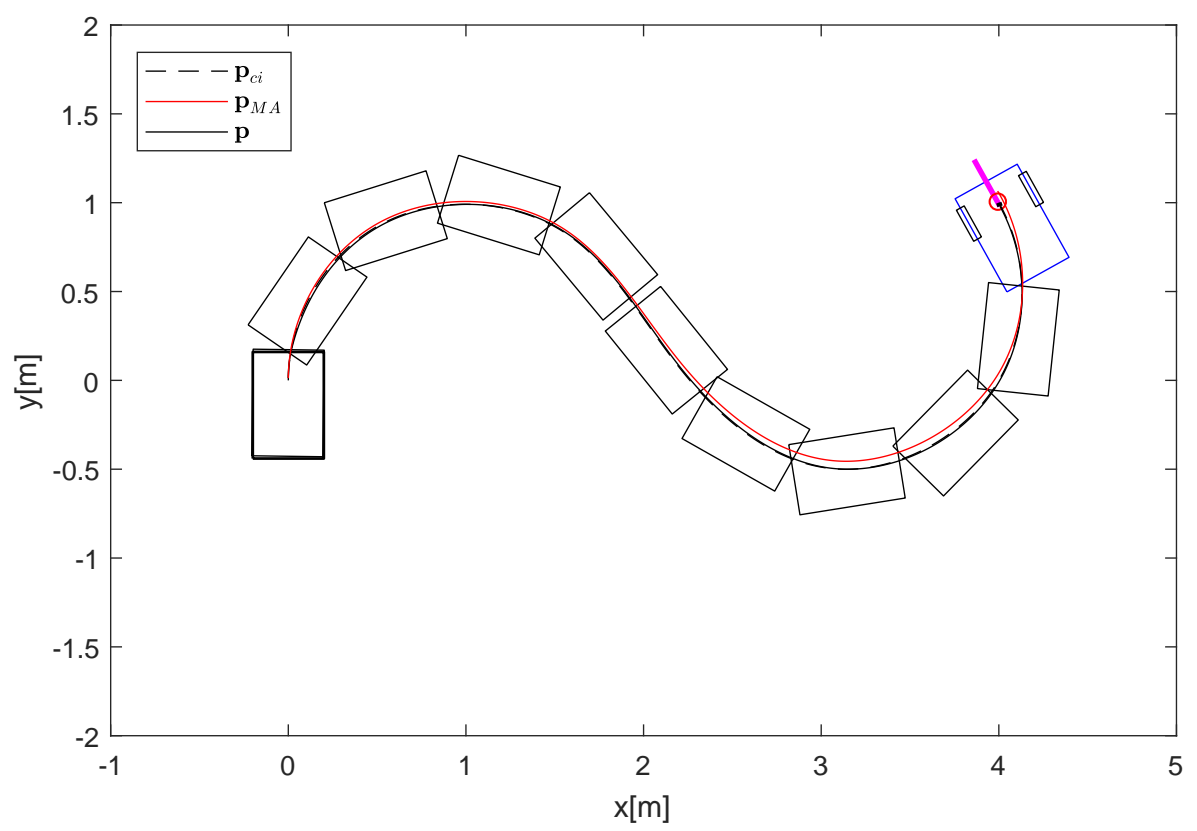

Figura 62 - Rastreamento FBL+PID no plano em uma Dubins Suave tipo RSL. Fonte: o autor.
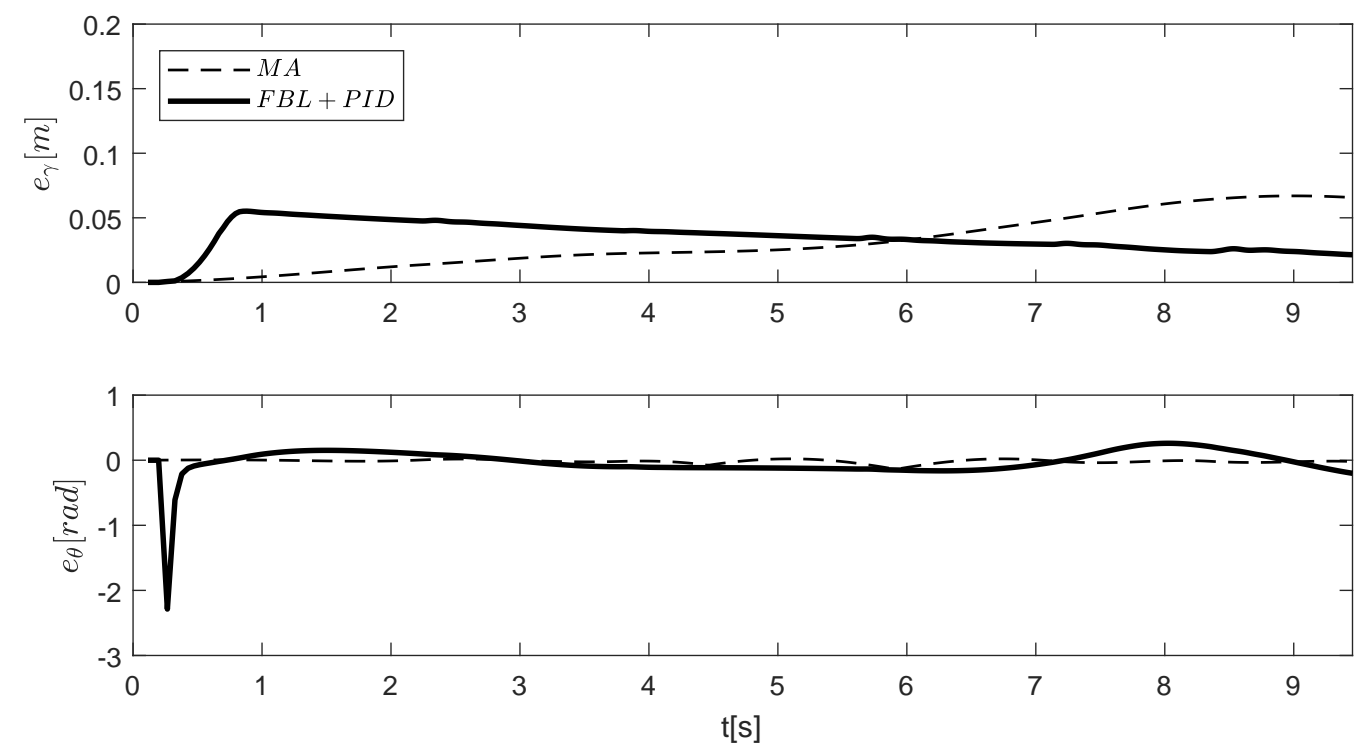

Figura 63 - Erros de rastreamento nas coordenadas alternativas em uma Dubins Suave tipo $R S L$.

Fonte: o autor.

Percebe-se nas três simulações um comportamento similar no que se diz respeito aos erros de rastreamento. Em particular, o erro linear $e_{\gamma}$ possui uma lenta convergência a zero no tempo. O erro linear final nas três simulações numéricas realizadas não chega a $e_{\gamma}\left(T_{f}\right)=0.02 \mathrm{~m}$. 
Esta convergência poderia ser acelerada aumentando-se o ganho $K_{\gamma}$. Porém, tentativas de se aumentar $K_{\gamma}$ para além de $K_{\gamma}=-100$ levaram a uma instabilidade no rastreamento durante o período transitório.

\subsection{CONCLUSÃO DO CAPÍTULO}

O resultado do algoritmo de Planejamento de Trajetória proposto por esta Tese no Capítulo 3 são os sinais ótimos de velocidade $v^{*}$ e de tensões dos motores $\mathbf{u}^{*}$, tais que o robô percorra um caminho dado de maneira eficiente.

Observa-se que a aplicação direta do sinal de tensões $\mathbf{u}^{*}$ no modelo do robô não resulta em uma reprodução eficaz do caminho originalmente dado como referência. Isto se deve ao fato de que erros de otimização - mesmo que pequenos - revelam-se relativamente grandes no final da integração numérica. Para corrigir este problema se faz necessária a presença de um controlador de Rastreamento de Trajetória.

O problema de Rastreamento de Trajetória se traduz em encontrar uma tensão dos motores do robô $\mathbf{u}$, tal que o robô siga um determinado caminho dado por pontos $\mathbf{p}_{c}$. Uma adaptação da abordagem "Siga a Cenoura" (RANKIN, 1997) é então utilizada para se propor um controlador de rastreamento de trajetória.

Propõe-se um controlador de dupla malha na qual o controle da cinemática do robô seja realizado por um controlador baseado em linearização por realimentação entrada-saída (feedback linearization - FBL), e o controle da dinâmica realizado por um controlador Proporcional-Integral-Derivativo (PID). O código em MatLab ${ }^{\circledR}$ do controle de malha dupla é apresentado no Apêndice B.4. Os resultados deste esquema de controle demonstraram-se satisfatórios no rastreamento de curvas Dubins Suaves. 



\section{CONCLUSÃO}

Este trabalho apresentou uma proposta de controle de movimento para um robô móvel sob rodas diferencial, com ênfase na otimização da eficiência do movimento, e cuja arquitetura é baseada em controle hierárquico aninhado.

O recorte do modelo hierárquico proposto possui três níveis distintos de controle: (i) o Planejador de Caminho, responsável por traçar a melhor rota no plano para o robô; (ii) o Planejador de Trajetória, cuja função é gerar um perfil de velocidade para o robô baseado em algum critério de otimização, e (iii) o Rastreador de Trajetória, que gera a ação de controle necessária para que o robô consiga percorrer o caminho sob o perfil de velocidade imposto.

Apesar destes níveis de controle estarem em ordem hierárquica, o desenvolvimento do texto desta Tese teve uma ordem diferenciada com o intuito de apresentar mais resultados na etapa de Planejamento de Trajetória.

O Capítulo 3 apresenta o nível de Planejamento de Trajetória, dado um determinado caminho. O método de otimização proposto por Verscheure et al. (2008), e posteriormente adaptado por Lipp e Boyd (2014), abrange apenas modelos de robôs que não possuem restrições não-holonômicas em seu modelo cinemático. Isto se deve ao fato de que dinâmicas sub-atuadas não impõem restrições de igualdade no modelo de otimização convexa proposto.

A primeira contribuição desta Tese é a adaptação deste método levando-se em consideração uma configuração alternativa. Enquanto na configuração generalizada clássica a postura do robô é definida por um vetor em $\mathbb{R}^{3}$, nesta configuração alternativa o vetor que representa a postura do robô no plano possui duas funções parametrizadas - de avanço e de orientação. Como o problema de otimização considera uma curva parametrizada pré-determinada para o movimento do robô, a utilização desta configuração alternativa permite que o modelo do robô uniciclo diferencial possa ser imposto como restrição neste método de otimização.

O conceito de eficiência usado nesta Tese considera duas grandezas distintas: tempo e energia. Estas grandezas são objetivos a serem minimizados em um problema de otimização convexa, ponderados por um coeficiente. Como são inversamente proporcionais, a fronteira de Pareto gerada pelos pontos ótimos parametrizados pelo coeficiente de ponderação se revelou sendo uma função exponencial em resultados experimentais. A segunda contribuição deste trabalho é a determinação dessa característica e a implementação de um algoritmo para sintonizar o coeficiente de ponderação, tendo como parâmetro uma razão de custo entre tempo e energia. 
O Planejador de Trajetória apresentado no Capítulo 3 otimiza a velocidade e o sinal de controle para qualquer curva de classe $C^{2}$ no plano. No Capítulo 4 se discute a utilização da geodésica de Dubins como caminho ótimo. Percebe-se experimentalmente que curvas de Dubins não podem ser utilizadas como referência de caminho para o método de otimização proposto. Isto porque as curvas de Dubins possuem descontinuidade em sua primeira derivada e, portanto, geram matrizes mal-condicionadas na dinâmica de segunda ordem do robô.

A proposta de suavização da geodésica de Dubins é a terceira contribuição desta Tese. Resultados experimentais de suavização de uma curva de Dubins específica mostram que o condicionamento da matriz da dinâmica de segunda ordem do robô reduz para 0,001\% depois da suavização, enquanto o erro máximo entre a geodésica original e a suavizada não passa de $0,026 \mathrm{~m}$.

Por fim, o Capítulo 5 apresenta uma análise de controle em malha aberta de Rastreamento de Trajetória considerando os sinais de tensão otimizados pelo nível de Planejamento de Trajetória. Resultados experimentais mostram que os erros de rastreamento presentes no controle em malha aberta não apresentam eficácia - em uma curva específica, o erro máximo equivale a quase um terço da largura do robô.

Para resolver o problema de Rastreamento de Trajetória, um esquema de controle de dupla malha é apresentado como contribuição final deste trabalho. Uma malha externa, baseada em linearização por realimentação entrada-saída (feedback linearization) é responsável pelo controle da cinemática do robô uniciclo diferencial, cuja ação de controle busca eliminar as não-linearidades presentes nas suas equações cinemáticas; enquanto uma malha interna controla a dinâmica do robô, relativa à relação entre a velocidade do robô no plano e as tensões elétricas em seus motores, por um controle Proporcional-Integral-Derivativo digital em forma de velocidade.

Os experimentos com três curvas Dubins Suave do tipo $L S L, R S R$ e $R S L$ mostram que, apesar de uma certa saturação de controle e consequente atraso no início do processo de rastreamento, o robô segue o caminho de maneira satisfatória.

A Figura 64 ilustra um diagrama de blocos com os três níveis de abstração e os seus subsistemas propostos nesta Tese, assim como ilustrações gráficas dos seus sinais intermediários.

O Planejador de Missão - nível hierárquico superior a este recorte - apresenta como missão as posturas inicial $\boldsymbol{\xi}_{0}$ e final $\boldsymbol{\xi}_{F}$ ao sistema. São então gerados waypoints de uma curva Dubins relaxada, com uma distância de aproximadamente $1 \mathrm{~m}$ entre eles. A suavização baseada em Spline Grampeada então interpola estes waypoints em uma série de $N$ pontos $\mathbf{p}_{i}$ que define um caminho por onde o robô deve seguir. Este caminho suavizado é muito próximo da geodésica entre as duas posturas. 


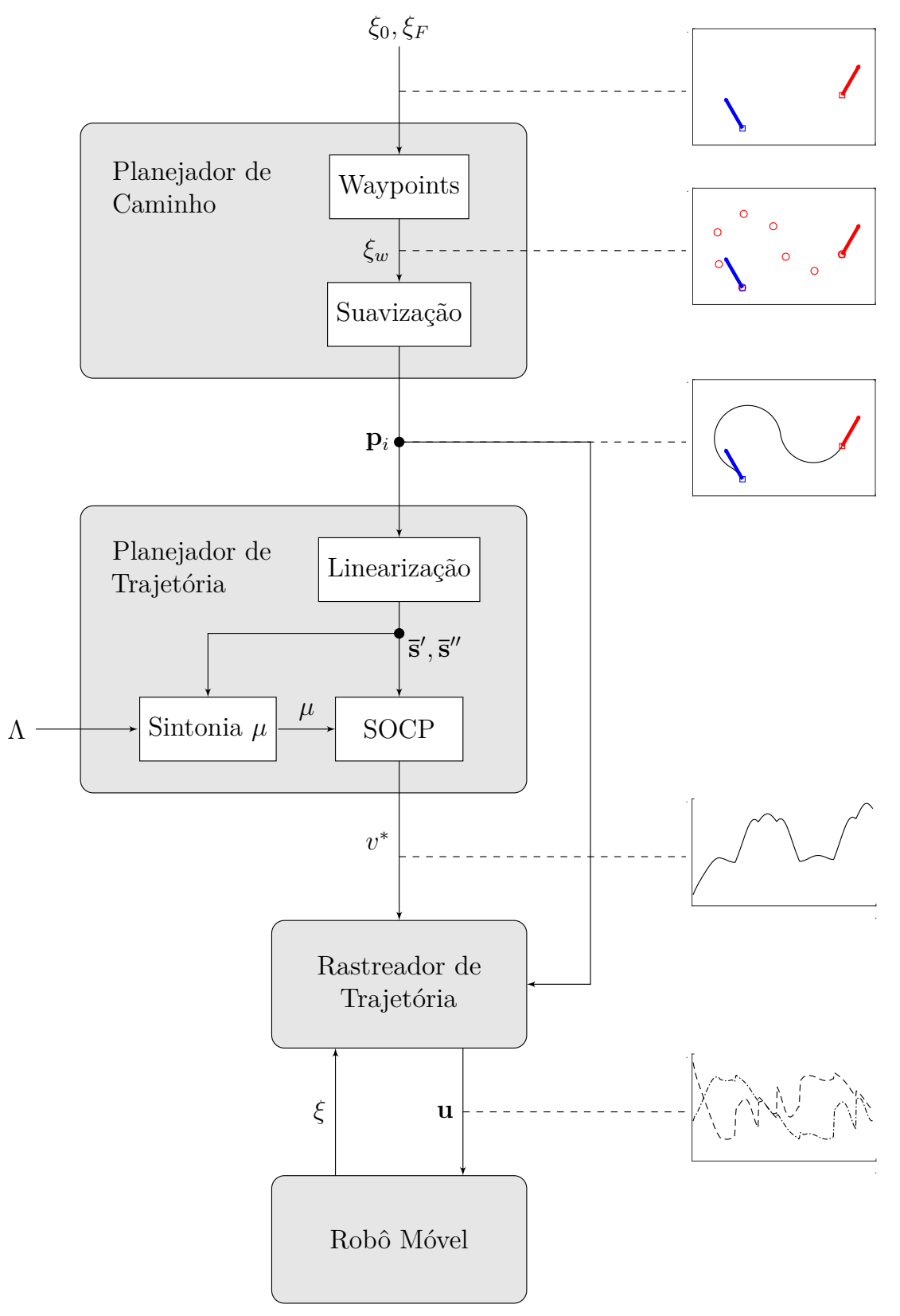

Figura 64 - Diagrama de blocos da Arquitetura de Controle de Movimento.

Fonte: o autor.

O Planejador de Trajetória então realiza uma transformação no espaço de configuração e lineariza as derivadas de primeira e segunda ordem dos pontos médios do caminho $\overline{\mathbf{s}}^{\prime}$ e $\overline{\mathbf{s}}^{\prime \prime}$. Então uma programação cônica de segunda ordem (SOCP) é executada com uma função objetivo ponderada entre tempo e energia, e cujo coeficiente de ponderação é dado por um algoritmo de sintonia. Este parâmetro, escolha do usuário baseada em uma relação de custo financeiro, é a razão entre o custo da energia e do tempo.

A velocidade ótima $v^{*}$, resultado da otimização de trajetória e o caminho geométrico são referência para um controlador de dupla malha FBL+PID que gera as tensões dos motores do robô para que este convirja geometricamente para um "Ponto Cenoura". 


\subsection{PUBLICAÇÕES DECORRENTES DA TESE}

- O controlador Feedback Linearization para o controle da cinemática do robô foi proposto e seus resultados apresentados no COBEM-2015.

SERRALHEIRO, W.; MARUYAMA, N.; TANNURI, E. Motion Control of an Underactuated Wheeled Mobile Robot: A Kinematic Input-Output Linearization Approach. In: 23rd International Congress of Mechanical Engineering (COBEM). Rio de Janeiro: ABCM, 2015. ISSN: 2176-5480.

- A transformação do espaço de configuração do robô, a linearização discreta e o algoritmo de otimização SOCP, assim como seus resultados foram apresentados no ICINCO-2017.

SERRALHEIRO,W; MARUYAMA, N. Time-Energy Optimal Trajectory Planning over a Fixed Path for a Wheeled Mobile Robot. In: 14th International Conference on Informatics in Control, Automation and Robotics (ICINCO). Madrid: INSTICC, 2017. p. 239-246. ISBN: 978-989-758-264-6

- Os resultados prévios do artigo apresentado no ICINCO-2017 juntamente com o algoritmo de sintonia presentes no Capítulo 3 foram submetidos ao JINT.

SERRALHEIRO, W.; MARUYAMA, N.; SAGGIN, F. Auto Tunned Time-Energy Optimization for a Wheeled Mobile Robot Trajectory Planning. Journal of Intelligent \& Robotic Systems, Springer (Submetido em 30/11/2017).

\subsection{TRABALHOS FUTUROS}

Durante este trabalho surgiram algumas perguntas que não comprometeram a completude dos objetivos traçados, mas que despertam interesse em respondê-las como futuros trabalhos.

O modelo dinâmico do robô que foi utilizado nesta Tese é um modelo simplificado, apenas com termos de segunda ordem. De fato, esta escolha se deveu a algumas dúvidas quanto à formulação original do método proposto por Verscheure et al. (2008), apresentadas no Apêndice A.

Em uma revisão da literatura, o recente trabalho de Reynoso-Mora, Chen e Tomizuka (2016) considera uma reformulação do modelo dinâmico do robô e apresenta um relaxamento nas restrições da otimização convexa que aparentemente superam o problema da formulação original. Por precaução, preferiu-se simplificar o modelo dinâmico nesta 
Tese; mas pretende-se em trabalhos futuros explorar com mais profundidade um modelo dinâmico mais completo.

Outra proposta para um trabalho futuro é adaptar o Controlador de Movimento para uma sequência de posturas $\boldsymbol{\xi}_{0}, \boldsymbol{\xi}_{1}, \ldots, \boldsymbol{\xi}_{F}$. Esta sequência gera um conjunto concatenado de curvas Dubins que compõe uma geodésica entre cada uma dessas posturas.

Pretende-se, por fim, implementar este Controlador em um robô uniciclo diferencial real, e comparar resultados de simulação numérica com resultados físicos. 



\section{REFERÊNCIAS}

AGUIAR, A.; HESPANHA, J. Trajectory-tracking and path-following of underactuated autonomous vehicles with parametric modeling uncertainty. IEEE Transactions on Automatic Control, IEEE, v. 52, n. 8, p. 1362-1379, 2007. Citado na página 49.

AICARDI, M. et al. Closed loop steering of unicycle-like vehicles via Lyapunov techniques. IEEE Robotics and Automation Magazine, IEEE, v. 2, n. March, p. 27-35, 1995. Citado 2 vezes nas páginas 41 e 48.

ALESSANDRETTI, A.; AGUIAR, A.; JONES, C. N. Trajectory-tracking and pathfollowing controllers for constrained underactuated vehicles using Model Predictive Control. In: EUROPEAN CONTROL CONFERENCE, 2013, Zurich. Proceedings... Zurich: IEEE, 2013. p. 1371-1376. Citado na página 49.

ALGER, B.; VOTION, J.; QIAN, C. Sampled-Data Output Feedback Control of Uncertain Nonholonomic Systems in Chained Forms with Applications to Mobile Robots. In: AMERICAN CONTROL CONFERENCE (ACC), 2014, Portland. Proceedings... Portland: IEEE, 2014. p. 4659-4664. Citado na página 48.

ANDERSEN, E.; ROOS, C.; TERLAKY, T. On implementing a primal-dual interior-point method for conic quadratic optimization. Mathematical Programming, Springer, v. 95, n. 2, p. 249-277, Feb 2003. Citado na página 65.

ARDESHIRI, T. et al. Convex Optimization Approach for Time-Optimal Path Tracking of Robots with Speed Dependent Constraints. In: IFAC WORLD CONGRESS, 2011, Milano. Proceedings... Milano: IFAC, 2011. v. 18, n. PART 1, p. 14648-14653. Citado na página 53.

ASHOORIRAD, M. et al. Model Reference Adaptive Path Following for Wheeled Mobile Robots. In: INTERNATIONAL CONFERENCE ON INFORMATION AND AUTOMATION, 2006, Shandong, China. Proceedings... Shandong, China: IEEE, 2006. p. 119-124. Citado na página 49.

ÅSTRÖM, K.; WITTENMARK, B. Computer-Controlled Systems: Theory and design, third edition. Upper Saddle River, New Jersey: Prentice Hall, 1997. 555 p. (Dover Books on Electrical Engineering Series). Citado na página 130.

BARTON, M. Controller development and implementation for path planning and following in an autonomous urban vehicle. 2001. 153 p. Tese (Doutorado) School of Aerospace, Mechanical and Mechatronic Engineering, University of Sydney, Sidney, 2001. Citado na página 129.

BENSON, S. J.; YE, Y.; ZHANG, X. Solving Large-Scale Sparse Semidefinite Programs for Combinatorial Optimization. SIAM Journal on Optimization, SIAM, v. 10, n. 2, p. 443-461, 2000. Citado na página 65.

BOBROW, J. E.; DUBOWSKY, S.; GIBSON, J. S. Time-Optimal Control of Robotic Manipulators Along Specified Paths. The International Journal of Robotics Research, SAGE, v. 4, p. 3-17, 1985. Citado na página 53. 
BORCHERS, B. CSDP, A C library for semidefinite programming. Optimization Methods and Software, Taylor \& Francis, v. 11, n. 1-4, p. 613-623, 1999. Citado na página 65.

BOYD, S. P.; VANDENBERGHE, L. Convex Optimization. Cambridge: Cambridge University Press, 2004. Citado 9 vezes nas páginas 39, 54, 55, 56, 57, 61, 64, 74 e 79.

BROCKETT, R. W. Asymptotic stability and feedback stabilization. In: BROCKETT, R. W.; MILlMAN, R. S.; SUSSMANN, H. J. (Org.). Differential Geometric Control Theory. Boston: Birkhauser, 1983. p. 181-191. Citado na página 48.

BURDEN, R.; FAIRES, J. Numerical Analysis. 9. ed. Boston: Cengage Learning, 2010. Citado 2 vezes nas páginas 111 e 112.

CANNY, J.; REGE, A.; REIF, J. An exact algorithm for kinodynamic planning in the plane. Discrete \& Computational Geometry, Springer, v. 6, n. 1, p. 461-484, 1991. Citado na página 52.

CANNY, J. et al. On the complexity of kinodynamic planning. In: 29TH ANNUAL SYMPOSIUM ON FOUNDATIONS OF COMPUTER SCIENCE, 1988, White Plains, NY, USA. Proceedings... White Plains, NY: IEEE, 1988. p. 306-316. Citado na página 52 .

CHEIKH, M. et al. A method for selecting pareto optimal solutions in multiobjective optimization. Journal of Informatics and Mathematical Sciences, v. 2, n. 1, p. 55-62, 2010. Citado na página 38.

CHO, G. S.; RYEU, J. K. An efficient method to find a shortest path for a car-like robot. Intelligent Control and Automation, Springer, v. 1, n. 1, p. 1000-1011, 2006. Citado 2 vezes nas páginas 38 e 105 .

CHWA, D. Sliding-Mode Tracking Control of Nonholonomic Wheeled Mobile Robots in Polar Coordinates. IEEE Transactions on Control Systems Technology, IEEE, v. 12, n. 4, p. 637-644, jul 2004. Citado na página 49.

CONSTANTINESCU, D.; CROFT, E. A. Smooth and time-optimal trajectory planning for industrial manipulators along specified paths. Journal of Field Robotics, Wiley Online Library, v. 17, n. 5, p. 233-249, 2000. Citado na página 53.

D'ANDREA-NOVEL, B.; CAMPION, G.; BASTIN, G. Structural Properties and Classification of Kinematic and Dynamic Models of Wheeled Mobile Robots. IEEE Transactions on Robotics and Automation, IEEE, v. 12, n. 1, p. 47-62, 1993. Citado na página 41.

DAS, T.; KAR, I. Design and implementation of an adaptive fuzzy logic-based controller for wheeled mobile robots. IEEE Transactions on Control Systems Technology, IEEE, v. 14, n. 3, p. 501-510, 2006. Citado na página 49.

DEBROUWERE, F. et al. Time-Optimal Path Following for Robots With Convex Concave Constraints Using Sequential Convex Programming. IEEE Transactions on Robotics, IEEE, v. 29, n. 6, p. 1485-1495, 2013. Citado na página 53.

DIXON, W. et al. Robust tracking and regulation control for mobile robots. International Journal of Robust and Nonlinear Control, Wiley Online Library, v. 10, p. 199-216, 2000. Citado na página 49. 
DONALD, B. et al. Kinodynamic Motion Planning. Journal of the Association for Computing Machinery, ACM, v. 40, n. 5, p. 1048-1066, 1993. Citado na página 52.

DUAN, J. et al. A path tracking control algorithm with speed adjustment for intelligent vehicle. In: IEEE CONFERENCE ON ROBOTICS AND BIOMIMETICS, 2013, Shenzhen, China. Proceedings... Shenzhen, China: IEEE, 2013. p. 2397-2402. Citado na página 129.

DUBINS, L. E. On Curves of Minimal Length with a Constraint on Average Curvature, and with Prescribed Initial and Terminal Positions and Tangents. American Journal of Mathematics, JSTOR, v. 79, n. 3, p. 497-516, 1957. Citado 4 vezes nas páginas 38, 47, 105 e 122.

EGERSTEDT, M. Motion Planning and Control of Mobile Robots. 2000. 192 p. Tese (Doutorado) - Department of Mathematics, Royal Institute of Technology, Stockholm, Sweden, 2000. Citado na página 52.

ENCARNAÇÃO, P.; PASCOAL, A. Combined trajectory tracking and path following control for dynamic wheeled mobile robots. In: IFAC WORLD CONGRESS, 2002, Barcelona. Proceedings... Barcelona: IFAC, 2002. Citado na página 49.

FIACCO, A.; MCCORMICK, G. Nonlinear Programming: Sequential Unconstrained Minimization Techniques. Philadelphia, PA: Society for Industrial and Applied Mathematics (SIAM), 1990. (Classics in Applied Mathematics). Citado na página 63.

FLETCHER, R. Practical Methods of Optimization. New York: John Wiley \& Sons, 1987. Citado na página 61.

GIESE, A. A Comprehensive Step-by-Step Tutorial on Computing Dubin's Curves. 2012. Disponível em: < http://gieseanw.files.wordpress.com/2012/10/dubins.pdf $>$. Citado 3 vezes nas páginas 38, 105 e 106.

GRANT, M.; BOYD, S. CVX: Matlab Software for Disciplined Convex Programming, version 2.1. 2014. Disponível em: <http://cvxr.com/cvx>. Citado 4 vezes nas páginas $65,79,86$ e 87.

GUROBI. Gurobi Optimizer. 2017. Disponível em: < https://www.ibm.com/analytics/ data-science/prescriptive-analytics/cplex-optimizer $>$. Citado na página 65 .

HELLSTROM, T.; RINGDAHL, O. Follow the Past: a path-tracking algorithm for autonomous vehicles. International Journal of Vehicle Autonomous Systems, Inderscience, v. 4, n. 2-4, p. 216-224, 2006. Citado na página 129.

HOGG, R. W. et al. Algorithms and sensors for small robot path following. In: IEEE INTERNATIONAL CONFERENCE ON ROBOTICS AND AUTOMATION, 2002, Washington, DC, USA. Proceedings... Washington, DC, USA: IEEE, 2002. v. 4, p. 3850-3857. Citado na página 129.

HUANG, H. C.; TSAI, C. C. Adaptive trajectory tracking and stabilization for omnidirectional mobile robot with dynamic effect. In: IFAC WORLD CONGRESS, 2008, Seoul, Korea. Proceedings... Seoul, Korea: IFAC, 2008. v. 17, p. 5383-5388. Citado na página 49. 
IBM. CPLEX Optimizer. 2017. Disponível em: < https://www.ibm.com/analytics/ data-science/prescriptive-analytics/cplex-optimizer $>$. Citado na página 65 .

ISIDORI, A. Nonlinear Control Systems. 3rd. ed. London: Springer, 1995. (Communications and Control Engineering). Citado 2 vezes nas páginas 48 e 129.

KANAYAMA, Y. et al. A Stable Tracking Control Method for a Non-Holonomic Mobile Robot. In: IEEE INTERNATIONAL WORKSHOP ON INTELLIGENT ROBOT AND SYSTEMS, 1991, Osaka, Japan. Proceedings... Osaka, Japan: IEEE, 1991. p. 1236-1241. Citado na página 48.

KHALIL, H. Nonlinear Systems. 2nd. ed. New Jersey: Prentice-Hall, 1996. Citado 3 vezes nas páginas 38, 48 e 129.

KIRK, D. Optimal Control Theory: An introduction. New Jersey: Englewood Cliffs, 1970. Citado na página 60.

KOLMANOVSKY, I.; MCCLAMROCH, N. H. Developments in nonholonomic control problems. IEEE Control Systems Magazine, IEEE, v. 15, p. 20-36, 1995. Citado na página 48.

LAVALLE, S. Planning Algorithms. New York, NY, USA: Cambridge University Press, 2006. Citado 3 vezes nas páginas 51, 53 e 105.

LAVALLE, S.; Kuffner, J.J., J. Randomized kinodynamic planning. The International Journal of Robotics Research, SAGE, v. 20, n. 5, p. 378-400, 2001. Citado na página 52.

LI, D.; YE, J. Adaptive Robust Control of Wheeled Mobile Robot with Uncertainties. In: IEEE INTERNATIONAL WORKSHOP ON ADVANCED MOTION CONTROL, 2014, Yokohama, Japan. Proceedings... Yokohama, Japan: IEEE, 2014. p. 518-523. Citado na página 49.

LIPP, T.; BOYD, S. Minimum-time speed optimisation over a fixed path. International Journal of Control, Taylor \& Francis, v. 87, n. 6, p. 1297-1311, 2014. Citado 7 vezes nas páginas 38, 54, 67, 72, 78, 102 e 139.

LIU, S.; SUN, D. Minimizing Energy Consumption of Wheeled Mobile Robots via Optimal Motion Planning. IEEE Transactions on Mechatronics, IEEE, v. 19, n. 2, p. 401-411, 2014. Citado na página 33.

LOBO, M. S. et al. Applications of second-order cone programming. Linear Algebra and its Applications, Eselvier, v. 284, n. 1-3, p. 193-228, 1998. Citado na página 80.

LOFBERG, J. Yalmip : a toolbox for modeling and optimization in matlab. In: IEEE INTERNATIONAL CONFERENCE ON ROBOTICS AND AUTOMATION, 2004, New Orleans. Proceedings... New Orleans: IEEE, 2004. p. 284-289. Citado na página 65.

LUCA, A. D.; ORIOLO, G.; SAMSON, C. Feedback Control of a Nonholonomic Car-like Robot. In: LAUMOND, J. (Org.). Robot Motion Planning and Control. New York: Springer, 1998. p. 171-253. Citado 2 vezes nas páginas 47 e 49. 
LUCA, A. D.; ORIOLO, G.; VENDITTELli, M. Control of wheeled mobile robots: An experimental overview. In: NICOSIA, S. et al. (Ed.). Ramsete - Articulated and Mobile Robotics for Services and Technologies. London: Springer, 2001. p. 181-226. Citado na página 50.

LUGO-CARDENAS, I. et al. Dubins path generation for a fixed wing UAV. In: INTERNATIONAL CONFERENCE ON UNMANNED AIRCRAFT SYSTEMS, 2014, Orlando, FL, USA. Proceedings... Orlando: IEEE, 2014. p. 339-346. Citado 2 vezes nas páginas 38 e 105.

MEYSTEL, A. Planning in a hierarchical nested autonomous control system. In: IEEE CONFERENCE ON DECISION AND CONTROL, 1986, Athens. Proceedings... Athens: IEEE, 1986. v. 727, p. 42-76. Citado na página 34.

MOSEK. The MOSEK optimization toolbox for MATLAB manual. 2017. 335 p. Disponível em: <http://docs.mosek.com/8.1/toolbox.pdf>. Citado na página 109.

MURPHY, R. Introduction to AI Robotics. Cambridge: MIT Press, 2000. Citado na página 34 .

MURRAY, R. M.; SASTRY, S. S. Steering nonholonomic systems in chained form. In: IEEE CONFERENCE ON DECISION AND CONTROL, 1991, Brighton, UK. Proceedings... Brighton, UK: IEEE, 1991. p. 1121-1126. Citado na página 48.

PEARSON, A. Efficiency vs. efficacy. ASHRAE Journal, v. 59, n. 4, p. 66, 2017. Citado na página 37.

PFEIFFER, F.; JOHANNI, R. A Concept for Manipulator Trajectory Planning. IEEE Journal on Robotics and Automation, IEEE, v. 3, n. 2, p. 115-123, 1987. Citado na página 53.

PHAM, Q.-C.; CARON, S.; NAKAMURA, Y. Kinodynamic Planning in the Configuration Space via Velocity Interval Propagation. Robotics: Science and Systems, Springer, Berlin, p. 306-316, 2013. Citado na página 52.

POURBOGHRAT, F.; KARLSSON, M. P. Adaptive control of dynamic mobile robots with nonholonomic constraints. Computers and Electrical Engineering, Elsevier, v. 28, n. 4, p. 241-253, 2002. Citado na página 49.

RANKIN, A. L. Development of Path Tracking Software for an Autonomous Steered-Wheeled Robotic Vehicle and its Trailer. 1997. 133 p. Tese (PhD) University of Florida, Florida, 1997. Citado 3 vezes nas páginas 38, 128 e 137.

REYHANOGLU, M. On the stabilization of a class of nonholonomic systems using invariant manifold technique. In: IEEE CONFERENCE ON DECISION AND CONTROL, 1995, New Orleans, LA, USA. Proceedings... New Orleans, LA, USA: IEEE, 1995. v. 3, n. December, p. 25-26. Citado na página 48.

REYNOSO-MORA, P.; CHEN, W.; TOMIZUKA, M. A convex relaxation for the timeoptimal trajectory planning of robotic manipulators along predetermined geometric paths. Optimal Control Applications and Methods, Wiley Online Library, v. 37, p. 12631281, 2016. Citado 4 vezes nas páginas 38, 54, 142 e 156. 
RIGATOS, G. G.; TZAFESTAS, C. S.; TZAFESTAS, S. G. Mobile robot motion control in partially unknown environments using a sliding-mode fuzzy-logic controller. Robotics and Autonomous Systems, Elsevier, v. 33, p. 1-11, 2000. Citado na página 49.

SAMSON, C. Control of Chained Systems Application to Path Following and TimeVarying Point-Stabilization of Mobile Robots. IEEE Transactions on Automatic Control, IEEE, v. 40, n. 1, p. 64-77, 1995. Citado na página 48.

SHANG, Y.; XIE, J. Global Stabilization of Nonholonomic Chained Form Systems with Input Delay. Abstract and Applied Analysis, Hindawi, v. 2014, p. 1-6, 2014. Citado na página 48.

SHILLER, Z. Time-Energy Optimal Control of Articulated Systems With Geometric Path Constraints. Journal of Dynamic Systems, Measurement, and Control, ASME, v. 118, n. 1, p. 139-143, 1996. Citado na página 53.

SHKEL, A. M.; LUMELSKY, V. Classification of the Dubins set. Robotics and Autonomous Systems, Elsevier, v. 34, n. 4, p. 179-202, 2001. Citado 2 vezes nas páginas 38 e 106 .

SICILIANO, B.; KHATIB, O. (Ed.). Springer Handbook of Robotics. Berlin, Heidelberg: Springer Berlin Heidelberg, 2008. Citado 2 vezes nas páginas 45 e 46.

SIEGWART, R.; NOURBAKHSH, I. Introduction to autonomous mobile robot. London: MIT Press, 2004. 336 p. Citado 3 vezes nas páginas 33, 43 e 46.

SLATER, M. Lagrange Multipliers Revisited. Cowles Foundation for Research in Economics, Yale University, 1959. Citado na página 60.

SLOTINE, J.; LI, W. Applied nonlinear control. 1st. ed. Englewood Cliffs: PrenticeHall, 1991. Citado 2 vezes nas páginas 48 e 50.

STANHOUSE, K.; KITTS, C.; MAS, I. Time-Energy Optimal Cluster Space Motion Planning for Mobile Robot Formations. In: WANG, G. (Ed.). Recent Advances in Robotic Systems. [S.1.]: InTechOpen, 2016. p. 141-163. Citado na página 37.

STURM, J. F. Using sedumi 1.02, a matlab toolbox for optimization over symmetric cones. Optimization Methods and Software, Taylor \& Francis, v. 11, n. 1-4, p. 625-653, 1999. Citado na página 65.

SUJIT, P.; SARIPALLI, S.; SOUSA, J. An evaluation of UAV path following algorithms. In: EUROPEAN CONTROL CONFERENCE (ECC), 2013, Zürich. Proceedings... Zürich, 2013. p. 3332-3337. Citado na página 129.

TAYEBI, a.; TADJINE, M.; RACHID, A. Invariant manifold approach for the stabilization of nonholonomic chained systems: Application to a mobile robot. Nonlinear Dynamics, Springer, v. 24, n. 2, p. 167-181, 2001. Citado na página 48.

TOH, K. C.; TODD, M. J.; TüTüNCü, R. H. Sdpt3 - a matlab software package for semidefinite programming, version 1.3. Optimization Methods and Software, Taylor \& Francis, v. 11, n. 1-4, p. 545-581, 1999. Citado na página 65.

TZAFESTAS, S. G. Introduction to Mobile Robot Control. London: Elsevier, 2014. 750 p. Citado 3 vezes nas páginas 46, 48 e 49. 
VERSCHEURE, D. et al. Time-energy optimal path tracking for robots: a numerically efficient optimization approach. In: IEEE INTERNATIONAL WORKSHOP ON ADVANCED MOTION CONTROL, 2008, Trento. Proceedings... Trento: IEEE, 2008. p. 727-732. Citado 7 vezes nas páginas 38, 53, 67, 72, 102, 139 e 142.

Time-optimal path tracking for robots: A convex optimization approach. IEEE Transactions on Automatic Control, IEEE, v. 54, n. 10, p. 2318-2327, 2009. Citado 5 vezes nas páginas 38, 53, 76, 77 e 155 .

WALKER, A. Dubins-Curves: an open implementation of shortest paths for the forward only car. 2008. Disponível em: < https://github.com/AndrewWalker/ Dubins-Curves $>$. Citado na página 106.

WATANABE, K. et al. Underactuated control for nonholonomic mobile robots by using double integrator model and invariant manifold theory. In: IEEE/RSJ INTERNATIONAL CONFERENCE ON INTELLIGENT ROBOTS AND SYSTEMS, 2010, Taipei, Taiwan. Proceedings... Taipei, Taiwan: IEEE, 2010. v. 2, n. 0, p. 2862-2867. Citado na página 48.

WIT, J. Vector pursuit path tracking for autonomous ground vehicles. 2000. 315 p. Tese (Doutorado) - Department of Mechanical Engineering, University of Florida, Florida, 2000. Citado na página 129.

WIT, J.; CRANE, C. D.; ARMSTRONG, D. Autonomous ground vehicle path tracking. Journal of Robotic Systems, Wiley Online Library, v. 21, n. 8, p. 439-449, aug 2004. Citado na página 129.

WRIGHT, S. Primal-Dual Interior-Point Methods. Philadelphia, PA: Society for Industrial and Applied Mathematics (SIAM), 1997. Citado 2 vezes nas páginas 63 e 64.

XIANG, X. et al. Path tracking: Combined path following and trajectory tracking for autonomous underwater vehicles. In: IEEE/RSJ INTERNATIONAL CONFERENCE ON INTELLIGENT ROBOTS AND SYSTEMS, 2011, San Francisco. Proceedings... San Francisco: IEEE, 2011. p. 3558-3563. Citado na página 49.

YANG, E. et al. Nonlinear tracking control of a carlike mobile robot via dynamic feedback linearization. In: CONTROL CONFERENCE, 2004, University of Bath, UK. Proceedings... Bath: UKACC, 2004. Citado na página 48.

YUN, X.; YAMAMOTO, Y. On feedback linearization of mobile robots: Technical reports (cis). Pennsylvania: University of Pennsylvania, 1992. 22 p. Citado na página 48.

ZHANG, Q.; LI, S.-R.; GAO, X.-S. Practical smooth minimum time trajectory planning for path following robotic manipulators. In: AMERICAN CONTROL CONFERENCE, 2013, Washington, DC. Proceedings... Washington, DC: IEEE, 2013. p. 2778-2783. Citado na página 53.

ZHEN-YING, L.; CHAO-LI, W. Robust stabilization of nonholonomic chained form systems with uncertainties. Acta Automatica Sinica, Elsevier, v. 37, n. 2, p. 129-142, 2011. Citado na página 48. 

Apêndices 



\section{APÊNDICE A - PROBLEMA DE FORMULAÇÃO}

O artigo de Verscheure et al. (2009), cuja simbologia difere da usada nesta Tese, considera a dinâmica completa do manipulador n-DOF com ângulos de juntas $\mathbf{q} \in \mathbb{R}^{n}$ em função de torques $\tau \in \mathbb{R}^{n}$ como sendo

$$
\boldsymbol{\tau}=\mathbf{M}(\mathbf{q}) \ddot{\mathbf{q}}+\mathbf{C}(\mathbf{q}, \dot{\mathbf{q}}) \dot{\mathbf{q}}+\mathbf{F}_{\mathbf{s}}(\mathbf{q}) \operatorname{sgn}(\dot{\mathbf{q}})+\mathbf{G}(\mathbf{q}),
$$

onde $\mathbf{M}(\mathbf{q}) \in \mathbb{R}^{n \times n}$ é uma matriz de massa positiva definida, $\mathbf{C}(\mathbf{q}, \dot{\mathbf{q}}) \in \mathbb{R}^{n \times n}$ é uma matriz que considera os efeitos de Coriolis e centrífuga, $\mathbf{F}_{\mathbf{s}}(\mathbf{q}) \in \mathbb{R}^{n \times n}$ uma matriz de torques de friç̧ão de Couloumb enquanto $\mathbf{G}(\mathbf{q}) \in \mathbb{R}^{n}$ denota um vetor de gravidade.

Para um dado caminho $\mathbf{q}(s), s \in[0,1]$ o tempo virtual, usando a regra da cadeia as derivadas da configuração são:

$$
\begin{aligned}
\dot{\mathbf{q}}(s) & =\mathbf{q}^{\prime}(s) \dot{s}, \\
\ddot{\mathbf{q}}(s) & =\mathbf{q}^{\prime}(s) \ddot{s}+\mathbf{q}^{\prime \prime}(s) \dot{s}^{2}
\end{aligned}
$$

Substituindo (A.2) e (A.3) em (A.1), a manipulação algébrica deveria ser:

$$
\begin{aligned}
& \boldsymbol{\tau}=\mathbf{M}(\mathbf{q})\left[\mathbf{q}^{\prime}(s) \ddot{s}+\mathbf{q}^{\prime \prime}(s) \dot{s}^{2}\right]+\mathbf{C}(\mathbf{q}, \dot{\mathbf{q}})\left[\mathbf{q}^{\prime}(s) \dot{s}\right]+\mathbf{F}_{\mathbf{s}}(\mathbf{q}) \operatorname{sgn}(\dot{\mathbf{q}})+\mathbf{G}(\mathbf{q}), \\
& \boldsymbol{\tau}=\left[\mathbf{M}(\mathbf{q}) \mathbf{q}^{\prime}(s)\right] \ddot{s}+\left[\mathbf{M}(\mathbf{q}) \mathbf{q}^{\prime \prime}(s)\right] \dot{s}^{2}+\left[\mathbf{C}(\mathbf{q}, \dot{\mathbf{q}}) \mathbf{q}^{\prime}(s)\right] \dot{s}+\left[\mathbf{F}_{\mathbf{s}}(\mathbf{q}) \operatorname{sgn}(\dot{\mathbf{q}})+\mathbf{G}(\mathbf{q})\right],
\end{aligned}
$$

que pode ser reescrita como

$$
\boldsymbol{\tau}=\mathbf{a}_{1}(s) \ddot{s}+\mathbf{a}_{2}(s) \dot{s}^{2}+\mathbf{a}_{3}(s) \dot{s}+\mathbf{a}_{4}(s) .
$$

Porém, no artigo a formulação dos autores é

$$
\boldsymbol{\tau}=\left[\mathbf{M}(\mathbf{q}) \mathbf{q}^{\prime}(s)\right] \ddot{s}+\left[\mathbf{M}(\mathbf{q}) \mathbf{q}^{\prime \prime}(s)+\mathbf{C}\left(\mathbf{q}, \mathbf{q}^{\prime}\right) \mathbf{q}^{\prime}(s)\right] \dot{s}^{2}+\left[\mathbf{F}_{\mathbf{s}}(\mathbf{q}) \operatorname{sgn}(\dot{\mathbf{q}})+\mathbf{G}(\mathbf{q})\right]
$$

que foi reescrita na equação (4) do artigo como

$$
\boldsymbol{\tau}=\mathbf{m}(s) \ddot{s}+\mathbf{c}(s) \dot{s}^{2}+\mathbf{g}(s),
$$

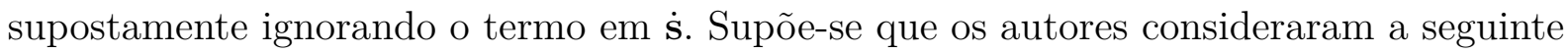
relação

$$
\left[\mathbf{C}(\mathbf{q}, \dot{\mathbf{q}}) \mathbf{q}^{\prime}(s)\right] \dot{s}=\left[\mathbf{C}\left(\mathbf{q}, \mathbf{q}^{\prime}\right) \mathbf{q}^{\prime}(s)\right] \dot{s}^{2}
$$

A matriz $\mathbf{C}(\mathbf{q}, \dot{\mathbf{q}})$ é uma matriz quadrada com seus termos em função de $\mathbf{q}$ e de $\dot{\mathbf{q}}$. Matematicamente não é correto considerar que substituindo termos $\dot{\mathbf{q}}(s)$ por $\mathbf{q}^{\prime}(s) \dot{s}$ garanta a igualdade

$$
\mathbf{C}(\mathbf{q}, \dot{\mathbf{q}}) \dot{s}=\mathbf{C}\left(\mathbf{q}, \mathbf{q}^{\prime}\right) \dot{s}^{2}
$$


O recente trabalho de Reynoso-Mora, Chen e Tomizuka (2016) considera a formulação da dinâmica do robô no caminho exatamente como a Equação (A.5).

Em seu trabalho, os autores consideram então três variáveis de otimização: $a(s)=\ddot{s}$, $b(s)=\dot{s}^{2}$ e $c(s)=\dot{s}$. Enquanto $b^{\prime}(s)=2 a(s)$ é uma restrição de igualdade linear no problema de otimização, a relação $c(s)=\sqrt{b(s)}$ é uma restrição de igualdade não-linear e, portanto, não-convexa. Como $b(s), c(s)>0$,

$$
\begin{aligned}
\sqrt{b(s)}=c(s) & \Longleftrightarrow \frac{1}{\sqrt{b(s)}}=\frac{1}{c(s)} \\
& \Longleftrightarrow \frac{1}{\sqrt{b(s)}} \leq \frac{1}{c(s)} \quad \text { e } \quad \frac{1}{c(s)} \leq \frac{1}{\sqrt{b(s)}} \\
& \Longleftrightarrow c(s)^{2}-b(s) \leq 0 \quad e \quad-c(s)^{2}+b(s) \leq 0
\end{aligned}
$$

A restrição de desigualdade $c(s)^{2}-b(s) \leq 0$ é convexa em $b(s)$ e $c(s), s \in[0,1]$. Porém, a restrição de desigualdade $-c(s)^{2}+b(s) \leq 0$ é côncava em seu domínio. Os autores propõem um relaxamento, desconsiderando a restrição côncava e simplesmente substituindo a restrição de igualdade não-linear $c(s)=\sqrt{b(s)}$ pela restrição de desigualdade convexa $c(s)^{2}-b(s) \leq 0$.

Os próprios autores consideram que não há garantias de que, "para todos os possíveis cenários, a solução ótima deste relaxamento irá resolver exatamente o problema não-convexo". Apesar disso, "todos os resultados numéricos apresentados pelo paper, a desigualdade $1 / \sqrt{b^{*}(s)} \leq 1 / c^{*}(s)$ foi atingida". 


\section{APÊNDICE B - CÓDIGOS EM MATLAB ${ }^{\circledR}$}

\section{B.1 TRANSFORMAÇÃO DE COORDENADAS}

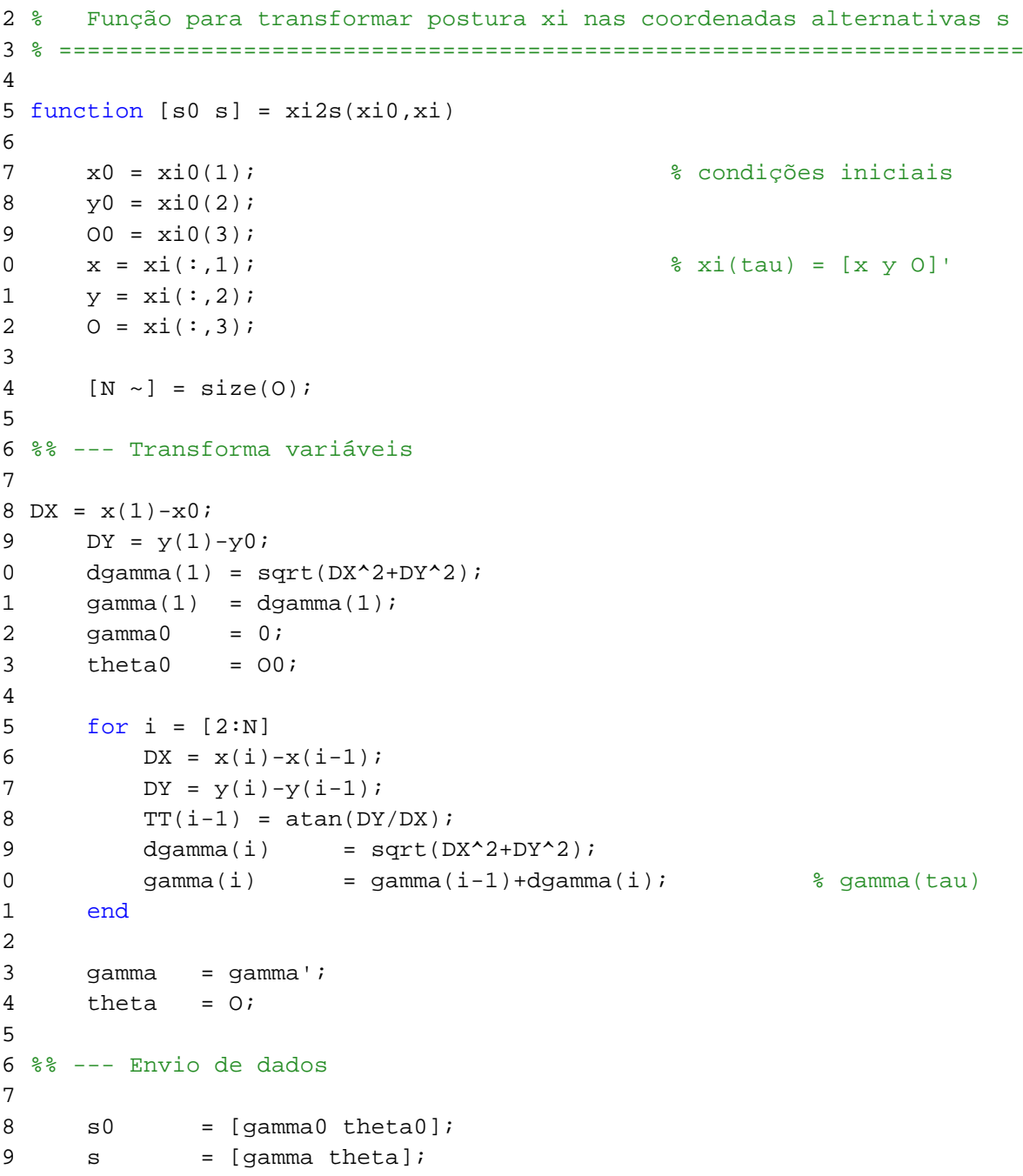




\section{B. $2 \mathrm{SOCP}$}

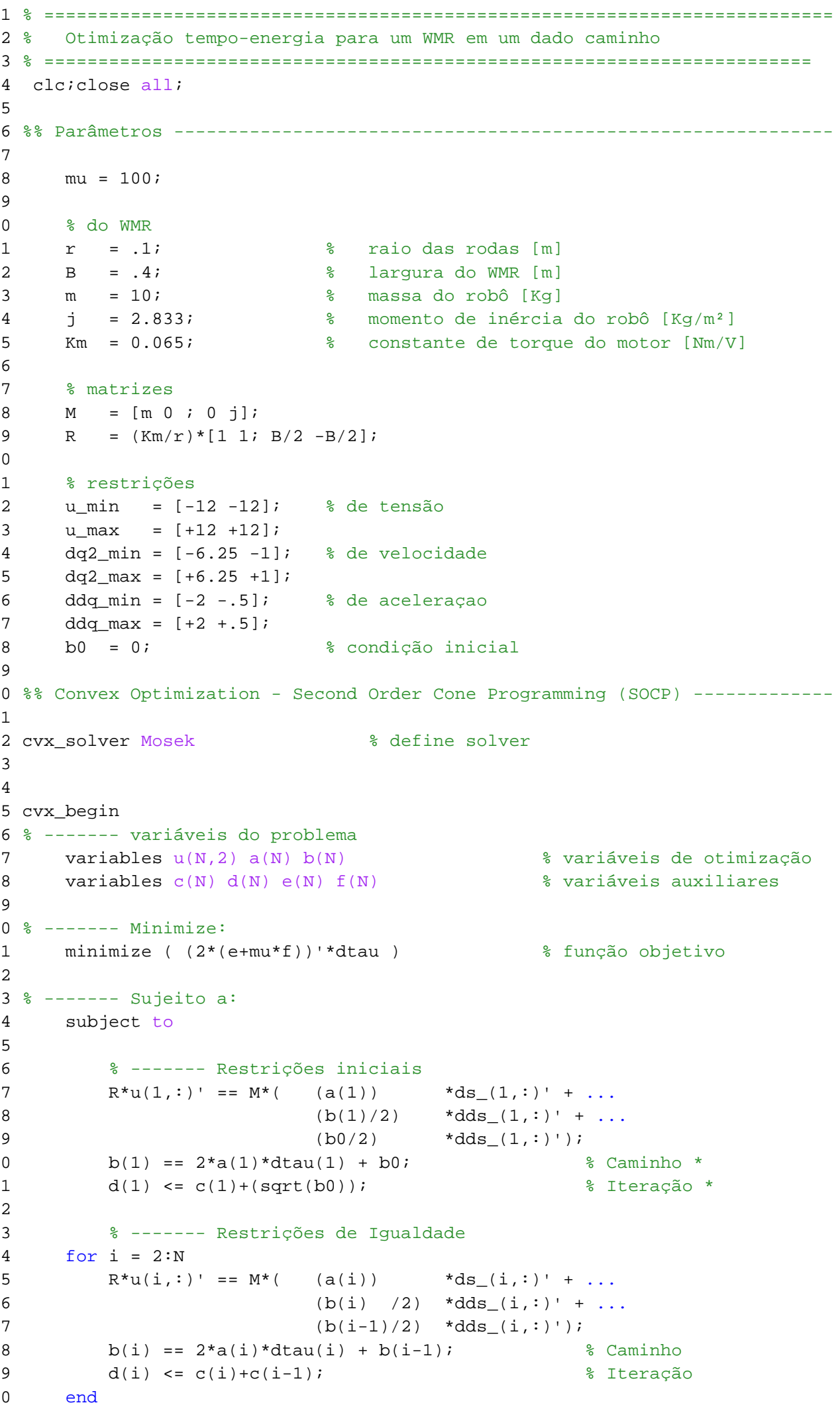




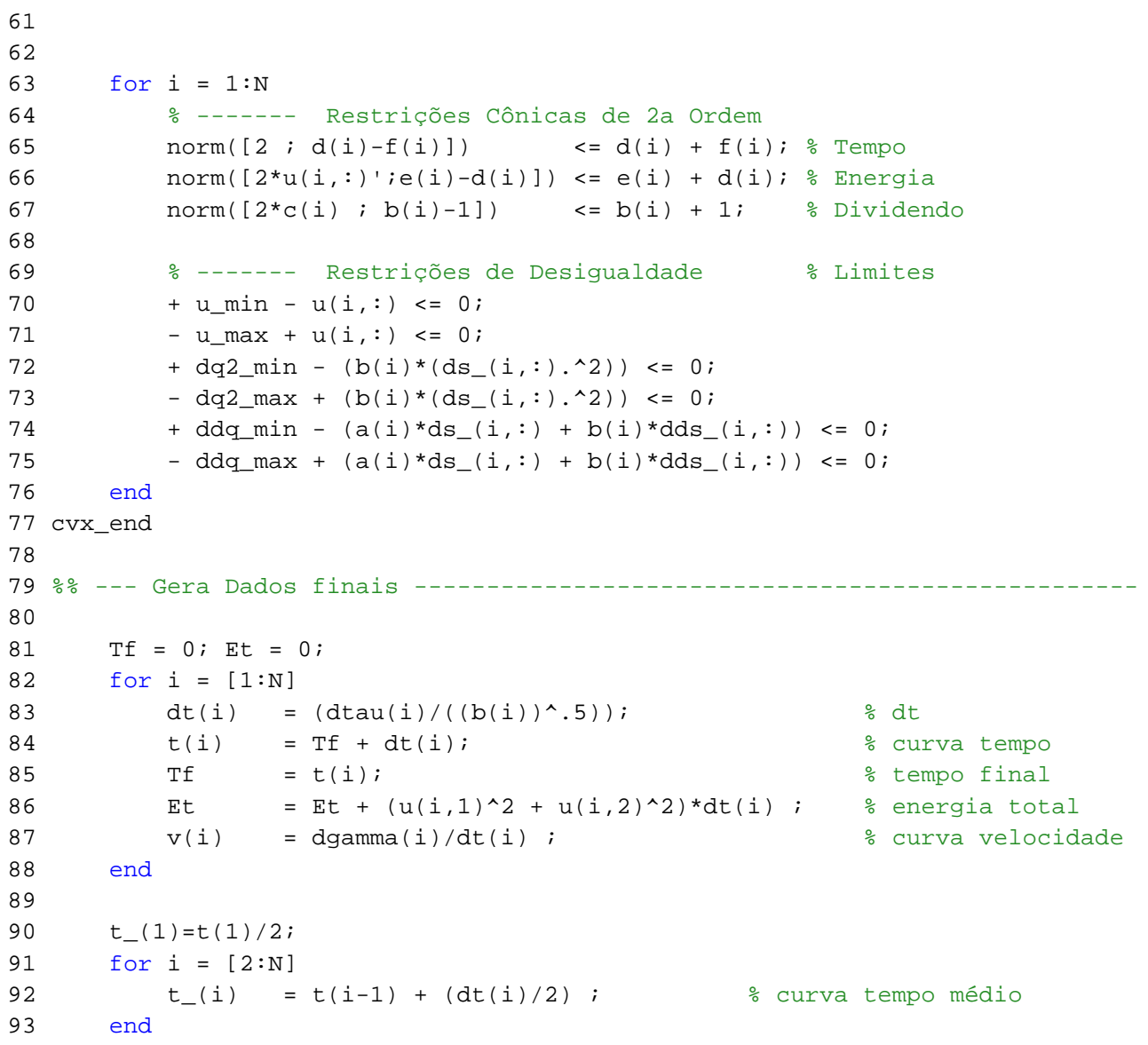




\section{B.3 CLAMPED SPLINE}

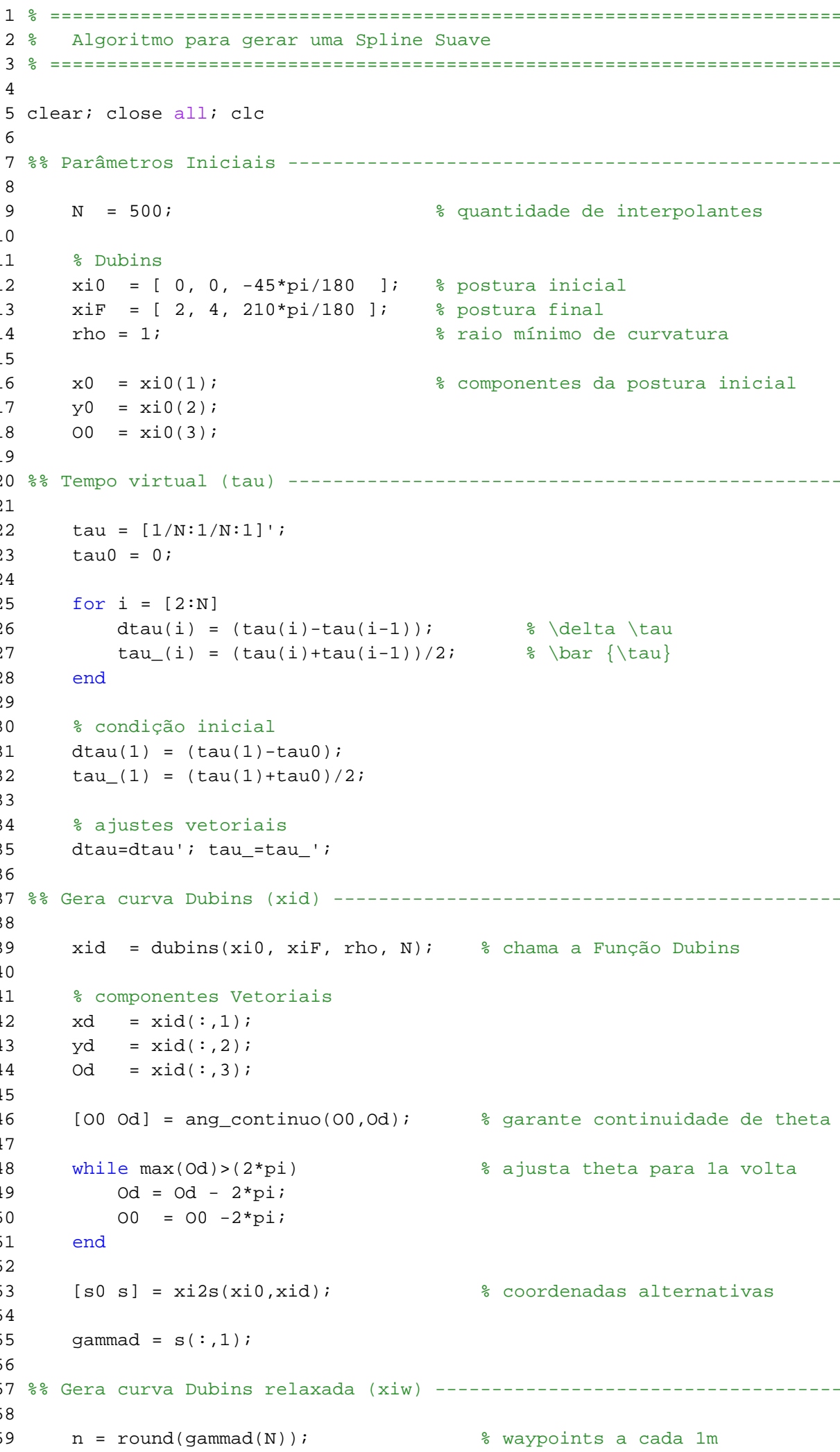




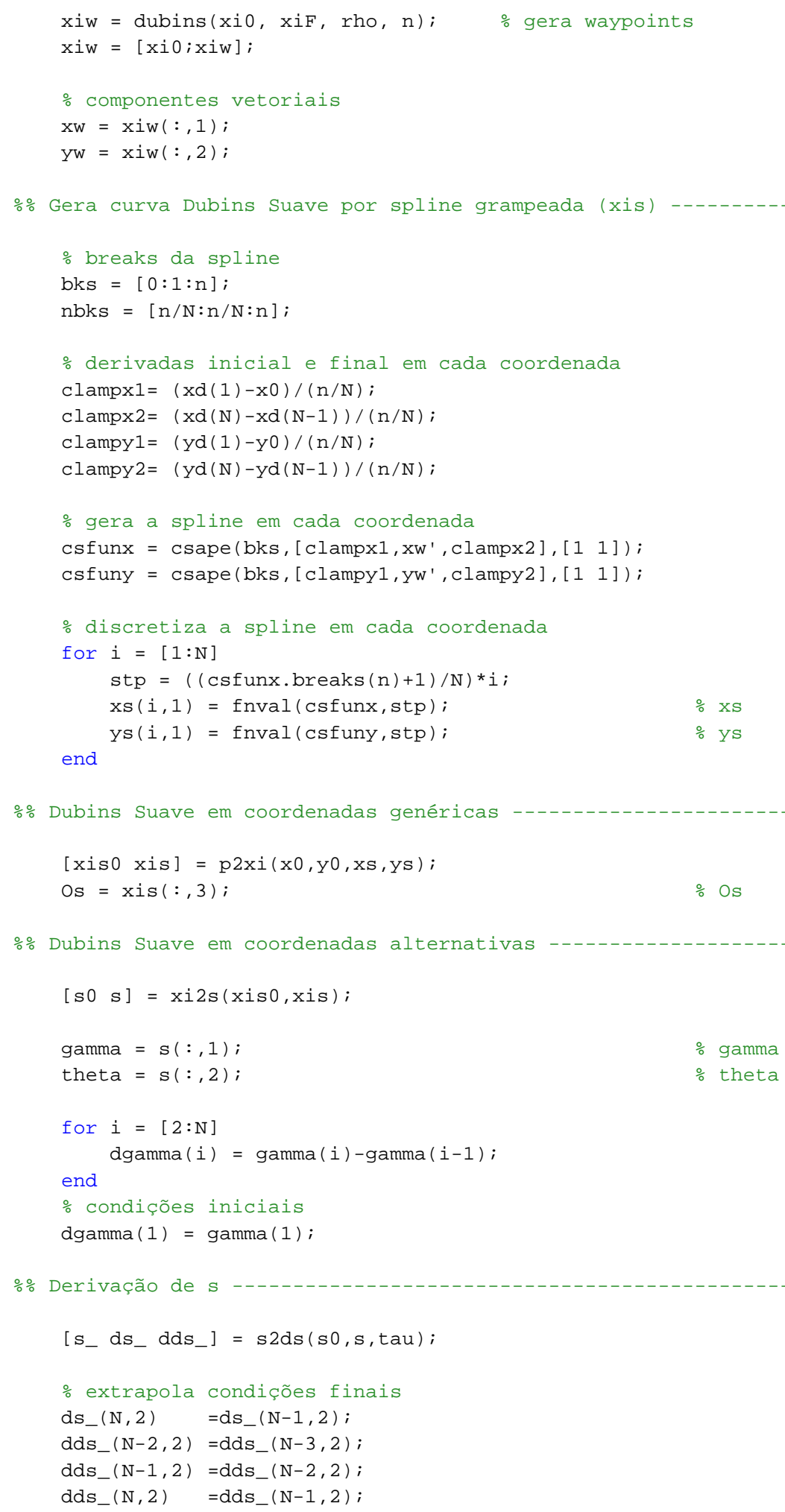




\section{B.4 CONTROLE DUPLA MALHA}

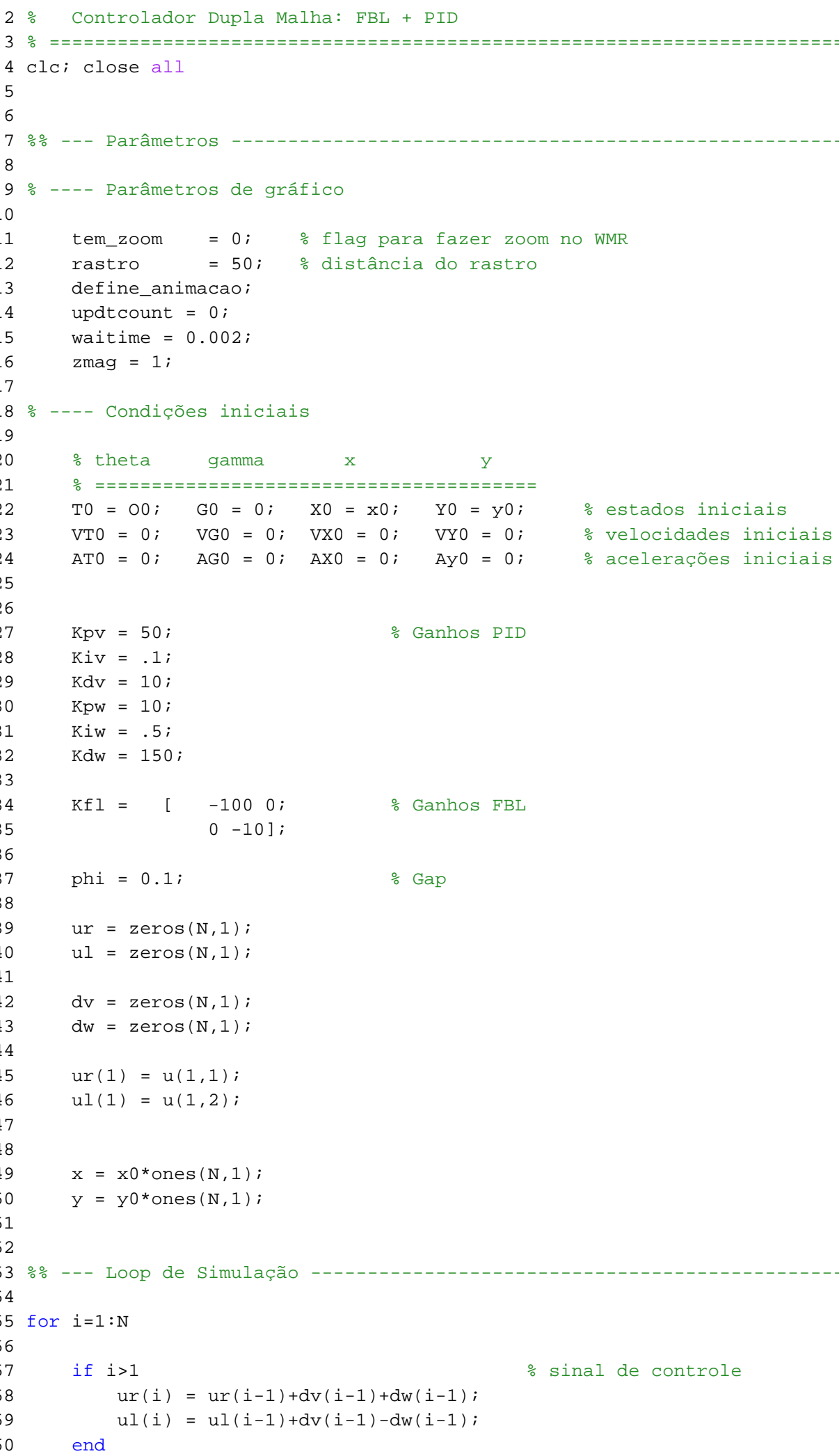




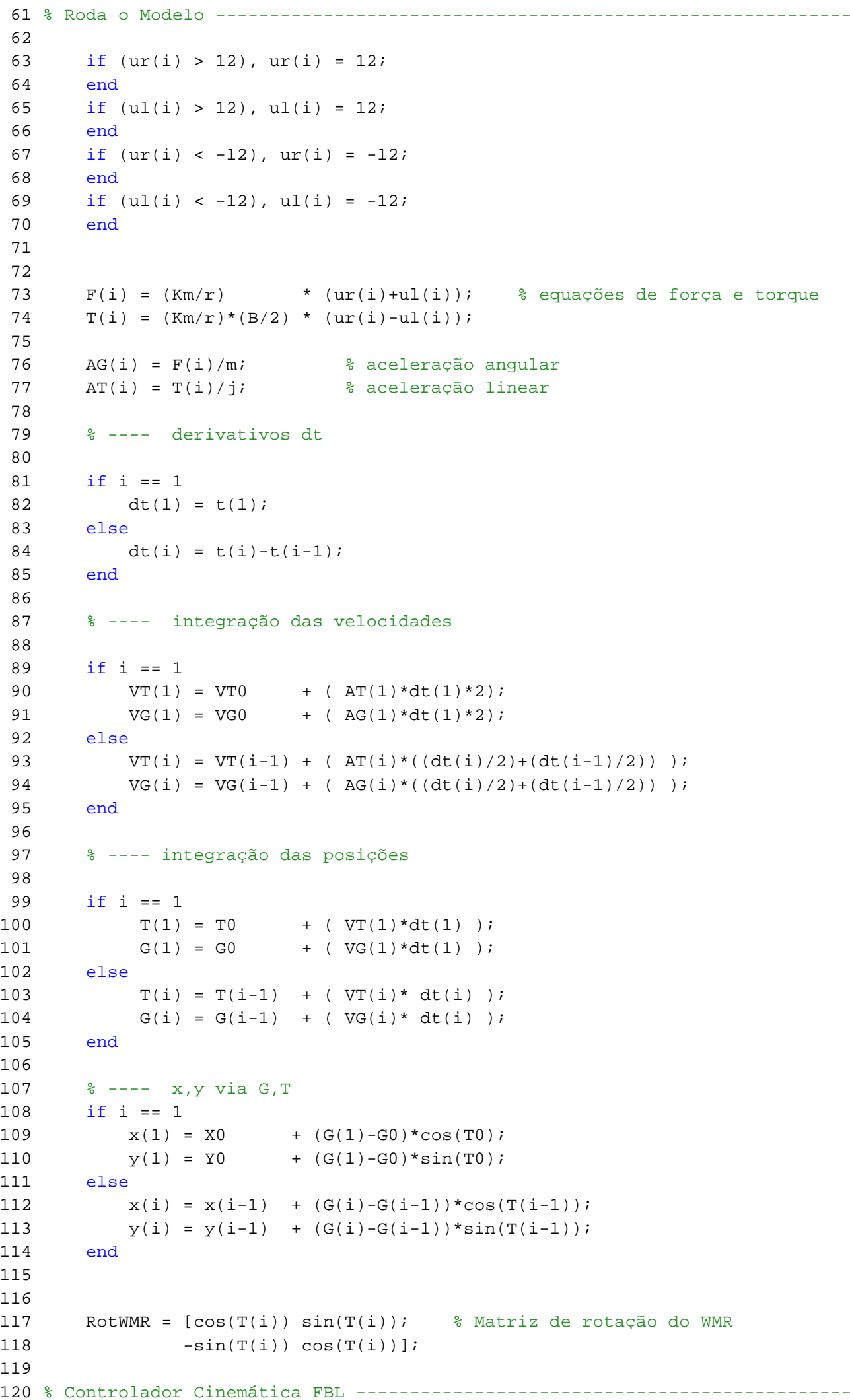




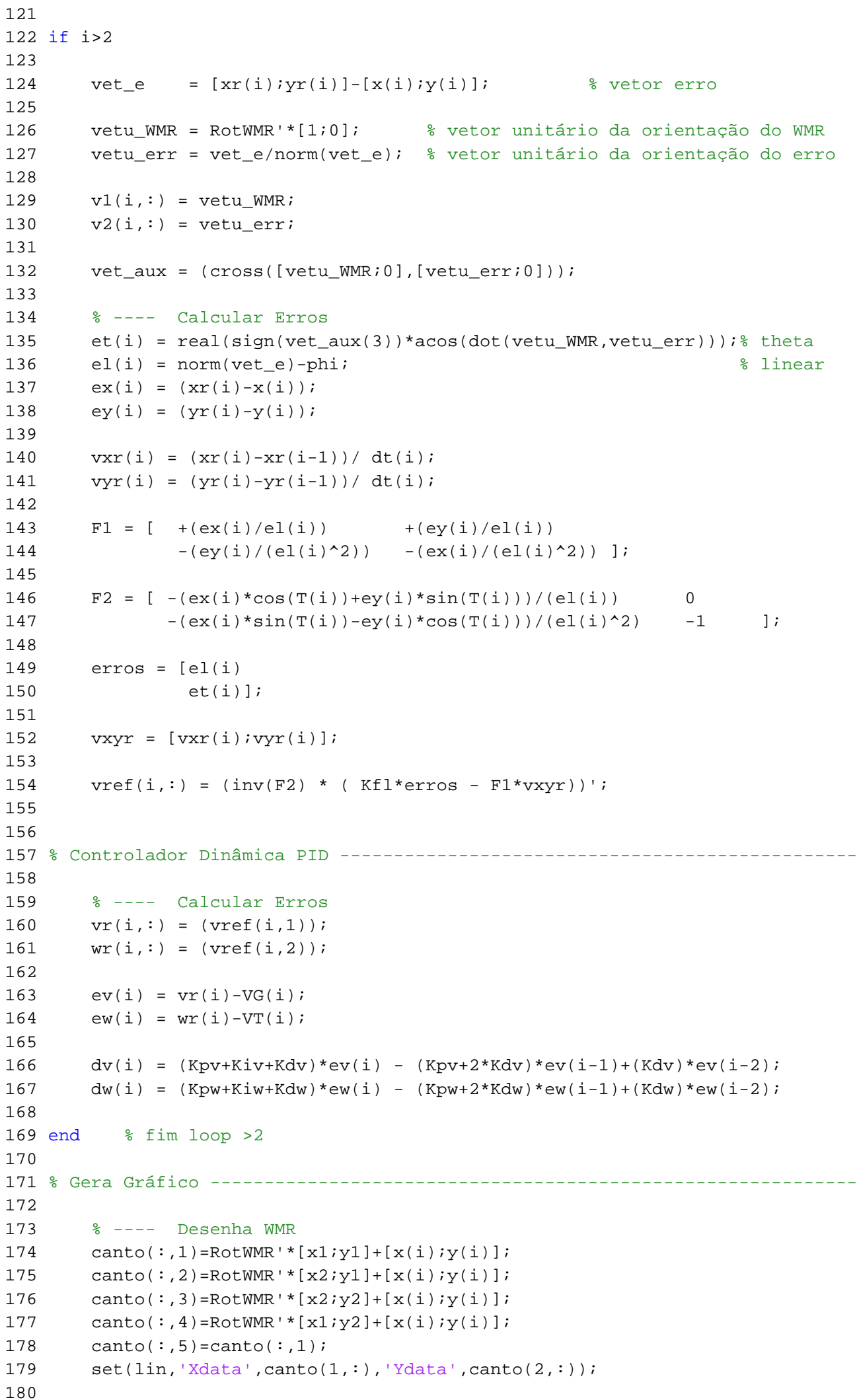




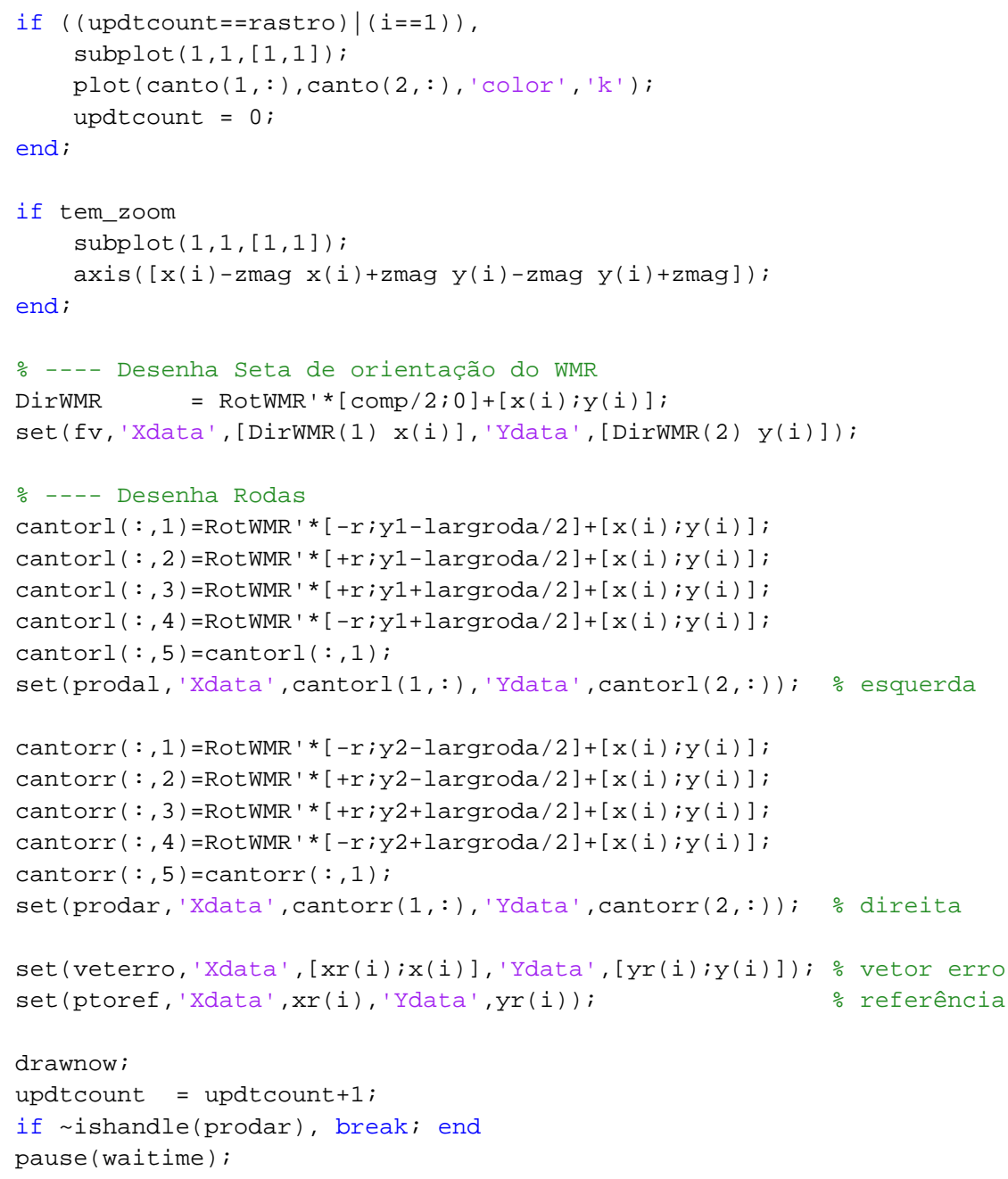

